\title{
SIMULATION OF A CROSSED-FIELD AMPLIFIER USING A MODULATED DISTRIBUTED CATHODE
}

\author{
by \\ Marcus Pearlman
}

\author{
A dissertation \\ submitted in partial fulfillment \\ of the requirements for the degree of \\ Doctor of Philosophy in Electrical and Computer Engineering \\ Boise State University
}

May 2017 
(c) 2017

Marcus Pearlman

ALL RIGHTS RESERVED 


\title{
DEFENSE COMMITTEE AND FINAL READING APPROVALS
}

of the dissertation submitted by

\author{
Marcus Pearlman
}

Dissertation Title: $\quad$ Simulation of a Crossed-Field Amplifier Using a Modulated Distributed Cathode

Date of Final Oral Examination: 15 February 2017

The following individuals read and discussed the dissertation submitted by student Marcus Pearlman, and they evaluated his presentation and response to questions during the final oral examination. They found that the student passed the final oral examination.

Jim Browning, Ph.D. Chair, Supervisory Committee

Wan Kuang, Ph.D. Member, Supervisory Committee

Kris A. Campbell, Ph.D. Member, Supervisory Committee

Yue Yin Lau, Ph.D. $\quad$ External Examiner

The final reading approval of the dissertation was granted by Jim Browning, Ph.D., Chair of the Supervisory Committee. The dissertation was approved by the Graduate College. 


\section{ACKNOWLEDGMENTS}

I would like to thank the Boise State University department of Electrical and Computer Engineering for financial support of this research. I thank the U.S. Air Force Office of Scientific Research for their financial support under Grant FA955012-C-0066. I also express my gratitude to TechX Corporation for their support of our work with Vsim and in particular David Smithe for his help. And, of course, I am immensely grateful to the review committee, Wan Kuang, Kris Campbell, Jim Browning, and Yue Ying Lau for their time and effort in validating this dissertation.

I also want to thank Janos Cserna for helping develop much of the experiment, specifically the X-Y stage used to measure the dispersion. I also wish to thank Kyle Straub and Tyler Rowe for their part in developing the experimental setup, and for their collaboration. 


\begin{abstract}
Current crossed-field amplifiers (CFAs) use a uniformly distributed electron beam, and in this work, the effects of using a spatially and temporally controlled electron source are simulated and studied. Spatial and temporal modulation of the electron source in other microwave vacuum electron devices have shown an increase in gain and efficiency over a continuous current source, and it is expected that similar progress will be made with CFAs. Experimentally, for accurate control over the electron emission profile, integration of gated field emitter arrays (GFEAs) as the distributed electron source in a crossed-field amplifier (CFA) is proposed.

Two linear format, 600 and $900 \mathrm{MHz}$ CFAs, which use GFEAs in conjunction with hop funnels as an electron source, were designed, modeled in VSim, and built at BSU. The hop funnels provide a way to control the energy of the electron beam separately from the sole potential and to protect the GFEA cathode. The dispersion of the meandering microstrip line slow wave circuit used in the device and the electron beam characteristics were measured and validated the simulation model, but experiments failed to show electron beam interaction with the electromagnetic wave due to insufficient current from the available cathode. To complete the research, a working CFA built at Northeastern University (NU) was modeled. The NU CFA was a linear format, device operating at $150 \mathrm{MHz}$, with $10 \mathrm{~W}$ of $\mathrm{RF}$ input power, and typically $150 \mathrm{~mA}$ of injected beam current. The electrically short device (6 slow wave wavelengths long) achieved $7 \mathrm{~dB}$ of gain. After validating the Vsim model against the experimental results, an electrically longer version (9 wavelengths)
\end{abstract}


was simulated with both an injected beam and distributed cathode. To model the distributed cathode computationally efficiently, where the emitted electron energy can be controlled separately from the sole potential, a new electron injection method was developed, using a divergence-free region.

Static electron emission profiles showed no improvement over the injected beam model but the temporally modulated cathode was found to significantly improve the performance. It was found that the temporal modulation could improve the small-signal-gain from $13 \mathrm{~dB}$ for an unmodulated source to $25 \mathrm{~dB}$ with an injected current of $150 \mathrm{~mA}$ and $0.1 \mathrm{~W}$ of $\mathrm{RF}$ drive power. This improvement is only likely to be observed for higher power devices $(>10 \mathrm{~kW}$ ) because of the additional RF drive power required by the GFEA, however. For larger RF drive powers, the improvements to gain become much smaller. With an RF drive power of $10 \mathrm{~W}$, the modulated cathode showed $9 \mathrm{~dB}$ of gain, and the injected beam variant showed $8 \mathrm{~dB}$. The signal-to-noise ratio (SNR) using the modulated cathode was consistently at least $15 \mathrm{~dB}$ higher than the SNR of the unmodulated cathode. This reduces the likelihood of excitation of unwanted modes. Even though this device showed small improvements to gain at large $\mathrm{RF}$ drive powers, it is proposed here that improvements to maximum power in higher power devices are likely, due to the inherent mode-locking mechanism of the modulated cathode, but this still needs to be confirmed. Previous research studying the effects of a modulated cathode in a magnetron and the improvements to the SNR shown here, show promise in this regard. 


\section{TABLE OF CONTENTS}

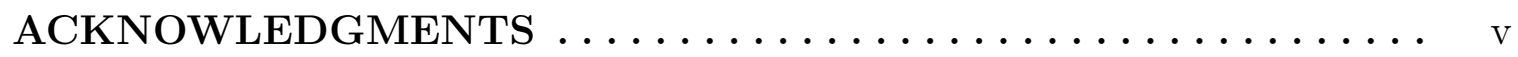

ABSTRACT $\ldots \ldots \ldots \ldots \ldots \ldots \ldots \ldots \ldots \ldots \ldots \ldots \ldots \ldots \ldots \ldots$

LIST OF TABLES $\ldots \ldots \ldots \ldots \ldots \ldots \ldots \ldots \ldots \ldots \ldots \ldots \ldots \ldots \ldots \ldots \ldots$

LIST OF FIGURES .....................

LIST OF ABBREVIATIONS $\ldots \ldots \ldots \ldots \ldots \ldots \ldots \ldots \ldots \ldots \ldots \ldots \ldots$ xxii

1 INTRODUCTION $\ldots \ldots \ldots \ldots \ldots \ldots \ldots \ldots \ldots \ldots \ldots \ldots \ldots \ldots \ldots$

1.1 Device Concept ......................... 4

1.2 Research Objectives and Contributions ............... 6

1.3 Overview of the Dissertation $\ldots \ldots \ldots \ldots \ldots \ldots \ldots \ldots$

2 BACKGROUND $\ldots \ldots \ldots \ldots \ldots \ldots \ldots \ldots \ldots \ldots \ldots \ldots \ldots \ldots \ldots \ldots$

2.1 Crossed-Field Amplifiers . . . . . . . . . . . . . . . . . . . . 11

2.2 Wave Velocities and Dispersion $\ldots \ldots \ldots \ldots \ldots \ldots \ldots \ldots \ldots$

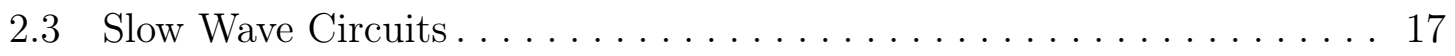

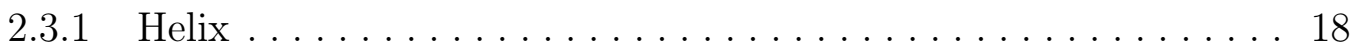

2.3 .2 Meander Line . . . . . . . . . . . . . . . . . . . . 20

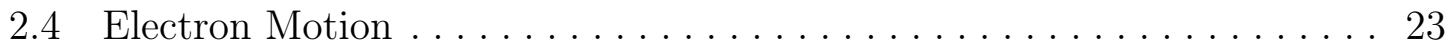

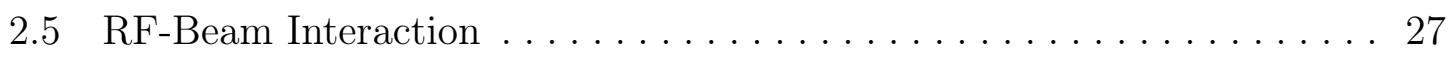


$2.5 .1 \quad$ Interaction Impedance . . . . . . . . . . . . . . . . 28

2.5.2 Beam Impedance . . . . . . . . . . . . . . . . . . . . . 30

2.5 .3 Theoretical Gain $\ldots \ldots \ldots \ldots \ldots \ldots \ldots \ldots \ldots \ldots \ldots$

2.6 Electron Sources . . . . . . . . . . . . . . . . . . . . . . . 34

2.6.1 Thermionic Cathodes ........................ 34

2.6.2 Emitting Sole $\ldots \ldots \ldots \ldots \ldots \ldots \ldots \ldots \ldots \ldots \ldots$

$2.6 .3 \quad$ Field Emitters $\ldots \ldots \ldots \ldots \ldots \ldots \ldots \ldots \ldots \ldots \ldots \ldots \ldots$

2.6.4 Hop Funnels . . . . . . . . . . . . . . . . . . . . . . 46

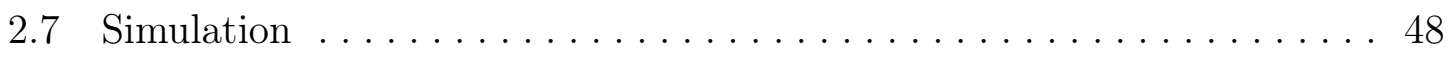

2.7 .1 COMSOL $\ldots \ldots \ldots \ldots \ldots \ldots \ldots \ldots \ldots \ldots \ldots \ldots \ldots$

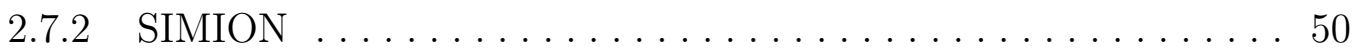

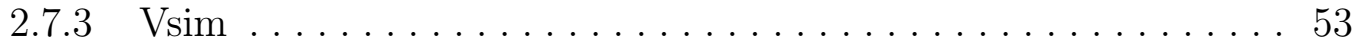

2.8 State of the Art in MVEDs $\ldots \ldots \ldots \ldots \ldots \ldots \ldots \ldots \ldots \ldots$

2.8.1 Cylindrical Emitting Sole CFAs _............... 59

2.8.2 Linear Format Injected Beam CFAs . . . . . . . . . . . . . 63

2.8.3 Linear Format CFA at Northeastern University . . . . . . . 66

2.8.4 Simulation of a Distributed Cathode in a Rising Sun Magnetron 69

2.8.5 Field Emitter Use in Microwave Vacuum Electron Devices .... . 70

3 Research Overview $\ldots \ldots \ldots \ldots \ldots \ldots \ldots \ldots \ldots \ldots \ldots \ldots$

3.1 Proposed Experimental Design . . . . . . . . . . . . . . . 75

3.1.1 Injected Beam Configuration Experiment . . . . . . . . . . 76

$3.1 .2 \quad$ Meander Line . . . . . . . . . . . . . . . . . . . . . 77

3.1 .3 Cathode ............................ 78

3.1.4 Distributed Cathode ......................... 79 
3.1.5 Sole/Hop Funnels . . . . . . . . . . . . . . . . . . . . . . 80

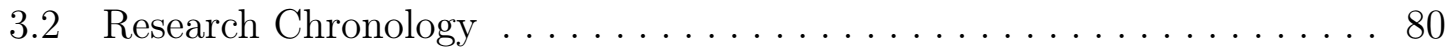

4 CFA Experiments and Measurements ............... 85

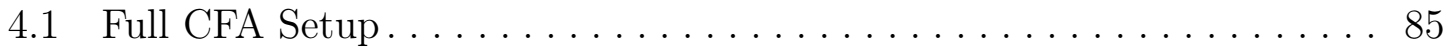

4.1.1 Vacuum Chamber and Electromagnets . . . . . . . . . . . . . 85

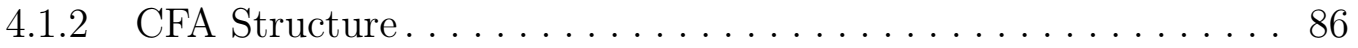

4.1.3 Slow Wave Circuit. . . . . . . . . . . . . . . . . 88

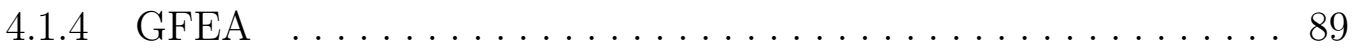

4.2 Meander Line Dispersion Measurements . . . . . . . . . . . . . . . . 91

$4.2 .1 \quad$ Experimental . . . . . . . . . . . . . . . . . . . . 91

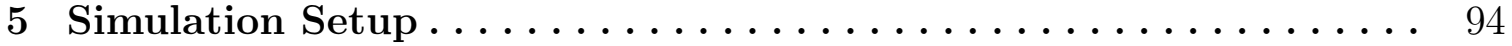

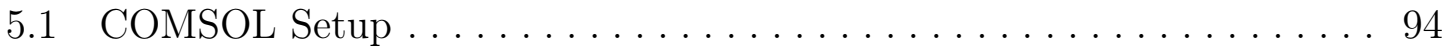

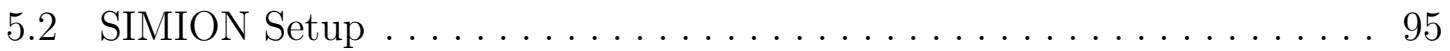

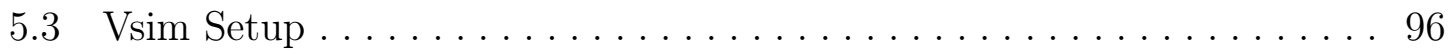

5.3 .1 The VSim Model . . . . . . . . . . . . . . . . . . . . . . . . 97

5.3 .2 Create the geometry . . . . . . . . . . . . . . . . . . . . . 102

5.3 .3 Non-Uniform Grid Model . . . . . . . . . . . . . . . . . . . . . 116

5.3 .4 Uniform Model . . . . . . . . . . . . . . . . . . . . . 121

5.3 .5 Injected Beam Cathode . . . . . . . . . . . . . . . . . . . 122

5.3 .6 Distributed Cathode . . . . . . . . . . . . . . . . . . . . 123

5.3.7 Distributed Cathode With Spatial and Time Varying Current . 138

6 Experimental and Simulated Results and Discussion of BSU CFA 146

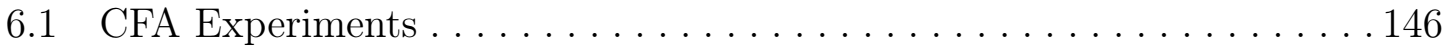




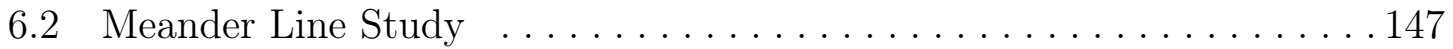

$6.2 .1 \quad$ S-Parameters. . . . . . . . . . . . . . . . . . . . . 147

$6.2 .2 \quad$ Dispersion . . . . . . . . . . . . . . . . . . . . . 148

6.2 .3 Discussion . . . . . . . . . . . . . . . . . . . . 155

6.3 Beam Optics: SIMION Comparison With Experiment. . . . . . . . . . 158

6.3 .1 Experiment . . . . . . . . . . . . . . . . . . . . 158

$6.3 .2 \quad$ SIMION . . . . . . . . . . . . . . . . . . . . 159

6.3 .3 Results ............................... 159

6.3 .4 Discussion . . . . . . . . . . . . . . . . . . . . 161

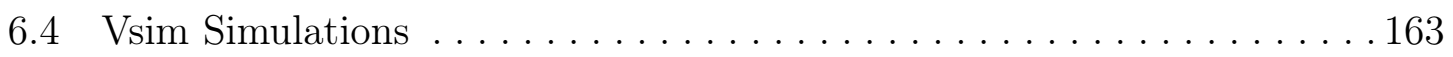

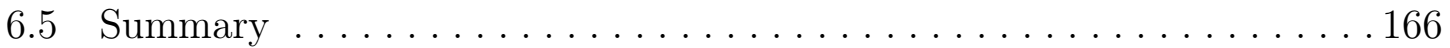

\section{Experimental and Simulated Results and Discussion of Northeastern} University CFA .......................... 168

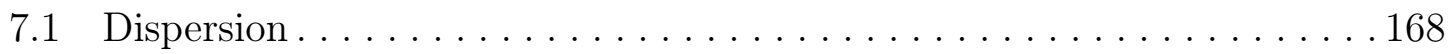

7.2 Electron Optics in Vsim . . . . . . . . . . . . . . . . . . 170

7.2.1 Cathode Placement . . . . . . . . . . . . . . . . . . . . . . . . . 171

7.2.2 Beam Electrode Potential . . . . . . . . . . . . . . . . . . . . 174

7.2.3 Cathode to Sole and Anode to Sole Voltage Study ........... 176

7.3 Injected Beam Characterization . . . . . . . . . . . . . . . . . 179

7.3.1 Optimum Magnetic Field . . . . . . . . . . . . . . . . 180

7.3.2 Beam Current Sweep . . . . . . . . . . . . . . . . . . . . . . 181

7.3.3 Bandwidth Sweep . . . . . . . . . . . . . . . . . . . . . . . 185

7.4 Injected Beam Studies . . . . . . . . . . . . . . . . . . . . . 186

7.4.1 Resolution Study . . . . . . . . . . . . . . . . . . . . . 187 
7.4.2 RF Power and Beam Power Gain Study . . . . . . . . . . . . . . . 188

7.4 .3 Gain vs. Circuit Length . . . . . . . . . . . . . . . . . . . . 196

7.5 Distributed Cathode Approximation Studies . . . . . . . . . . . . . . . 204

7.5.1 Cathode Approximation 1: Raised Cathode ............. . 205

7.5.2 Cathode Approximation Two: Segmented Cathode . . . . . . . . 207

7.5.3 Cathode Approximation Three: Raised Cathode With Approximated Current . . . . . . . . . . . . . . . . . . . 2212

7.6 Distributed Cathode Studies: Static . . . . . . . . . . . . . . . . 216

7.6.1 Uniform Emission Profile . . . . . . . . . . . . . . . 217

7.6 .2 Linear Emission Profile . . . . . . . . . . . . . . . . . . 221

7.6 .3 Summary . . . . . . . . . . . . . . . . . 221

7.7 Distributed Cathode Studies: Time Varying . . . . . . . . . . . . . . . . 222

7.7.1 Sine Wave Emission Profile Results . . . . . . . . . . . . . . . 223

7.7.2 Injected Beam Using Sine Wave Profile . . . . . . . . . . . . . . 225

7.7.3 Square Pulse Emission Profile Results . . . . . . . . . . . . . . . 226

7.7 .4 General Effects . . . . . . . . . . . . . . . . . . . . . 234

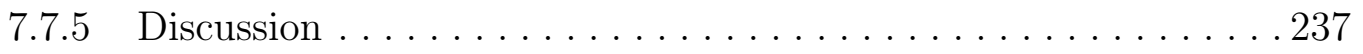

7.7 .6 Summary . . . . . . . . . . . . . . . . . . . . . . 242

7.8 Implementation on Higher Power CFA Designs . . . . . . . . . . . . . . 244

7.8 .1 Direct Implementation . . . . . . . . . . . . . . . . . 245

7.8 .2 Alternate Implementations . . . . . . . . . . . . . . . . 247

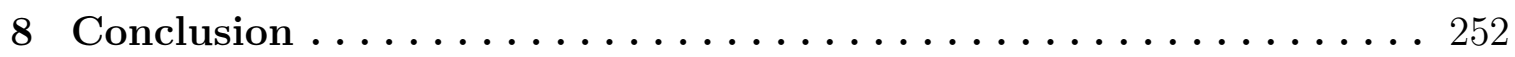

8.1 Model Verification . . . . . . . . . . . . . . . . . . . . . 252

8.2 General Observations . . . . . . . . . . . . . . . . . . . . . . . 254 
8.3 Distributed Cathode Observations . . . . . . . . . . . . . . . . . 255

8.3.1 Static Current Distributions .................... 255

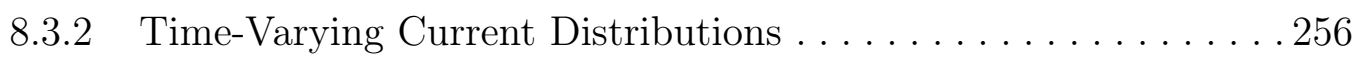

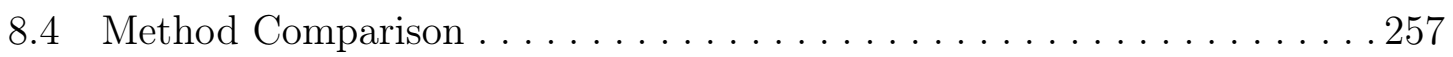

8.5 Modulated Distributed Cathode Importance . . . . . . . . . . . . . 259

$8.6 \quad$ Future Work . . . . . . . . . . . . . . . . . . . . . . . . . . . 259

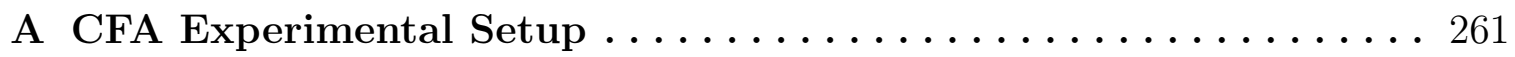

A.1 Measurement and Control Setup . . . . . . . . . . . . . . 261

A.1.1 CFA Ground $\ldots \ldots \ldots \ldots \ldots \ldots \ldots \ldots \ldots \ldots \ldots \ldots \ldots \ldots \ldots \ldots \ldots \ldots$

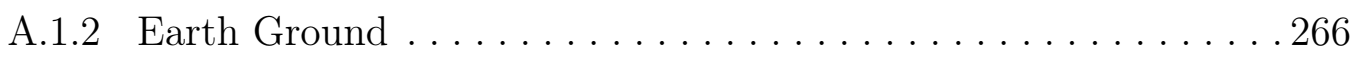

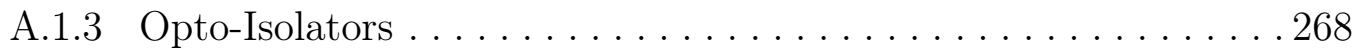

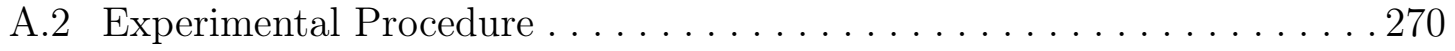

References................................272 


\section{LIST OF TABLES}

1.1 Summary of Microwave Vacuum Electron Devices $\ldots \ldots \ldots \ldots \ldots$

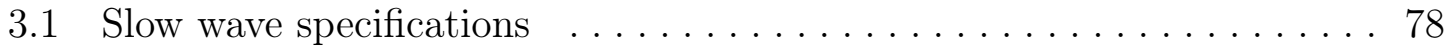

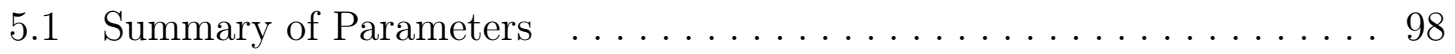

5.2 Slow wave circuit and CFA dimensions of the NU experiment and the VSim adjustments. Bold listed elements are parameters which are

altered in the VSim simulation to align well with the coarse grid. . . . 107 


\section{LIST OF FIGURES}

1.1 Range of applications of MVEDs in comparison with solid state devices. Reproduced with permission from $[2] . \ldots \ldots \ldots \ldots \ldots \ldots$

2.1 Cylindrical, injected beam, non-reentrant, backward wave crossed field

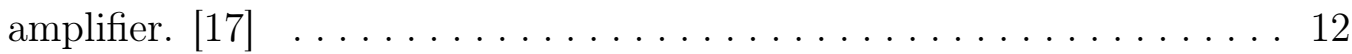

2.2 Cylindrical, emitting sole, reentrant, forward wave crossed field ampli-

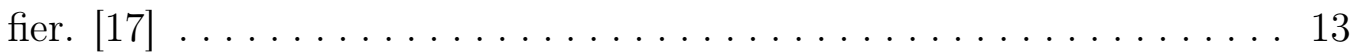

2.3 Linear format injected beam crossed field amplifier $[2] . \quad \ldots \ldots \ldots \ldots$. . 13

2.4 Dispersion diagram for waves traveling in either direction in a rectangular waveguide $[2] . \ldots \ldots \ldots \ldots \ldots \ldots \ldots \ldots \ldots$

2.5 Dispersion diagram for waves traveling in either direction in a periodically loaded rectangular waveguide $[2] . \quad \ldots \ldots \ldots \ldots \ldots$

2.6 Dispersion diagram for periodically loaded rectangular waveguide showing multiple harmonics $[2] . \ldots \ldots \ldots \ldots \ldots \ldots \ldots \ldots \ldots$

2.7 Helix (a) and view of helix cut at each $\mathrm{x}$ and unrolled (b).[7] . . . . . 19

2.8 Backward wave interaction at two turns per wavelength. [7] . . . . . . 20

2.9 A microstrip type meander line, showing a conducting meander circuit over a dielectric material and a ground plane. . . . . . . . . . . 21 
2.10 Electron forces and trajectories for (a) a constant electric field and no magnetic field, (b) a constant magnetic field into the page and no electric field, and (c) a constant electric field perpendicular to a constant magnetic field into the page. . . . . . . . . . . . . . . . 24

2.11 Electron trajectories for crossed electric and magnetic fields for various initial velocities $\left(u_{0}\right)$ [2]. $\omega_{c}$ is the cyclotron frequency defined by $\omega_{c}=q B / m$, where $q$ is the particle charge, $B$ is the magnitude of the magnetic field, and $m$ is the particle mass. . . . . . . . . . . 25

2.12 Voltage vs. magnetic field showing the operation region of magnetron

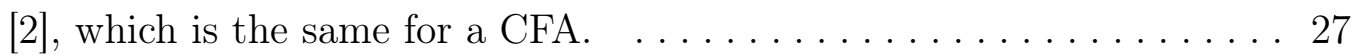

2.13 Motions of electrons due to the RF field in a rotating coordinate system of a CFA. Electrons in positive RF potentials move towards the sole as they give up energy, and move clockwise into the decelerating region (the region between positive and negative $\mathrm{RF}$ potentials where the electric field points clockwise). Electrons in negative RF potentials gain energy, and cycloid right back into the sole. Electrons in the decelerating regions remain in the region but move towards the sole as they give up energy. $[17] \ldots \ldots \ldots \ldots \ldots \ldots \ldots \ldots$

2.14 Plot of Pierce theory efficiency as a function of beam current for the

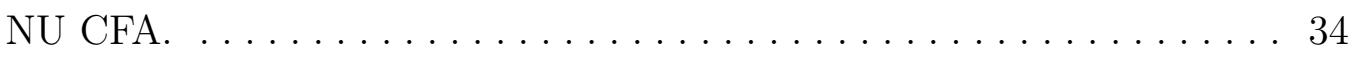

2.15 Energy Level Diagram near the surface of a metal $[2] \ldots \ldots \ldots \ldots \ldots$

2.16 Fermi-Dirac distribution for various temperatures. . . . . . . . 36

2.17 A typical secondary electron yield curve for an arbitrary material [2]. . 38

2.18 Diagram showing the 'multiplication' of electrons on the surface of an emitting sole cathode[2]. . . . . . . . . . . . . . . . . . 40 
2.19 Gated field emitter diagram showing the field enhancement near the

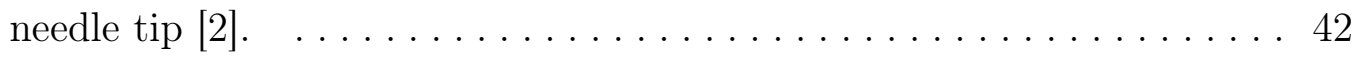

2.20 Energy level diagram at the surface of a material with and without an applied electric field $[24] . \quad \ldots \ldots \ldots \ldots \ldots \ldots \ldots \ldots \ldots$

2.21 The current density vs. the gate emitter voltage of the gated field

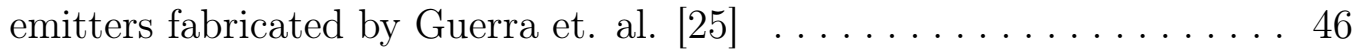

2.22 Hop funnels used in [37], showing the operation during (a) full electron transmission and (b) no transmission using the Lorentz 2E [60]

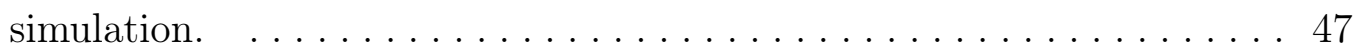

2.23 Hop funnel structure with sole electrode using Lorentz 2E [60] . . . . . 48

2.24 Yee grid showing the position of the various field components. Electric field components are on the middle of the edges and magnetic field

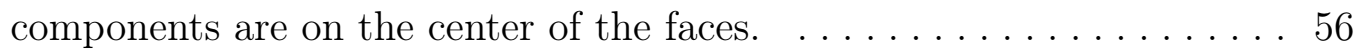

2.25 FDTD simulation flow $[71] \ldots \ldots \ldots \ldots \ldots \ldots \ldots \ldots \ldots$

2.26 Double helix coupled vane slow wave structure commonly used in

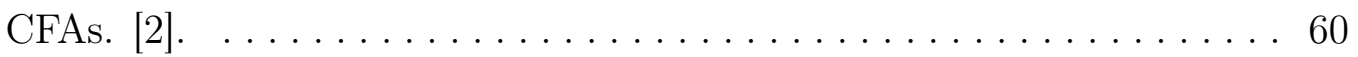

2.27 The current power capabilities of published CFA data. [2]. . . . . . 61

2.28 (a) conventional CFA comparison with a (b) cathode-driven and a (c)

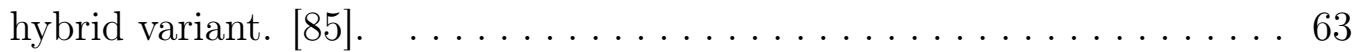

2.29 (a) Short and (b) long Kino electron gun schematics [87]. . . . . . . 64

2.30 Northeastern CFA schematic in Browning et. al. $[14,15] \ldots \ldots \ldots \ldots 6$

2.31 Northeastern CFA Gain vs. frequency plot in Browning et. al. $V_{a s}=$ $1250 \mathrm{~V}, B=5.2 \mathrm{mT}[14] \ldots \ldots \ldots \ldots \ldots \ldots$

2.32 Northeastern CFA Gain vs. Beam current plot in Browning et. al. $V_{A S}=1200 \mathrm{~V}, B=5.5 \mathrm{mT}[15] \ldots \ldots \ldots \ldots \ldots \ldots$ 
2.33 Northeastern CFA Gain vs. circuit with and without an electron beam in Browning et. al. $P_{r f}=10 \mathrm{~W}, V_{A S}=1200 \mathrm{~V}, B=5.5 \mathrm{mT}[15] \ldots \ldots .69$

2.34 GFEA matching circuit used in the TWT work [8], proposed by Calame

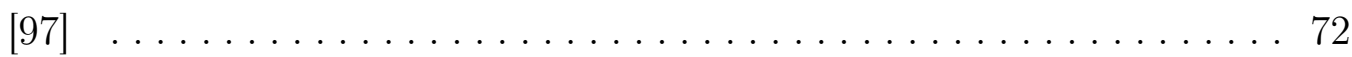

2.35 (top) The transparent cathode configuration with 6 cathode strips and (bottom) a solid cathode [98].

3.1 Schematic representation of the injected beam CFA design with dimen-

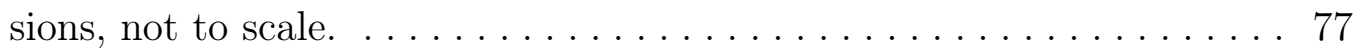

3.2 The diagram showing the meander line microstrip. A metal line meanders over a dielectric with thickness $H_{d}$ over a ground plane. . . . . 78

3.3 Schematic representation of the distributed cathode CFA design, not to scale. Electrons injected into the hop funnels are extracted though slits in the sole electrode. . . . . . . . . . . . . . . . . . . 79

3.4 Diagram outlining the research flow of the three CFA designs. The BSU experimental work was used to validate the simulation model, but all work on the BSU CFAs were terminated after determining the design was unfit. Results from the Northeastern CFA experimental work were also used to validate the simulation model, and the design was used for the distributed cathode studies.

4.1 Photograph of the electromagnets and the chamber system where the

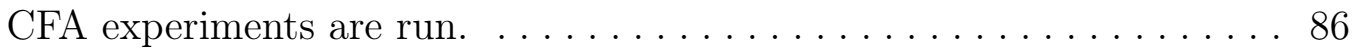

4.2 Schematic representation of the CFA design, not drawn to proportion. 87

4.3 Top down view of the CFA structure without the slow wave circuit. . 88 
4.4 Photograph of slow wave circuit SW2. A rectangular copper wire meanders on top of a Teflon dielectric which is on top of an aluminum ground plane. The copper wire is fixed to the ground plane by polypropylene screws. The input an output ports are SMA connectors which are connected to the copper wire by silver paste. . . . . . . . . . 89

4.5 Top down view of the CFA structure without the slow wave circuit and the end hats to show the PixTech cathode and the gate and emitter

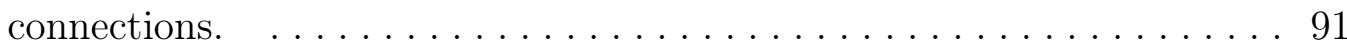

4.6 Photograph of the standing wave measurement setup. The slow wave circuit sits on top of an $\mathrm{x}-\mathrm{y}$ stage, and a coaxial cable connected to a spectrum analyzer on one end and the other end is placed right over the slow wave circuit with the center conductor exposed. . . . . . . . 93

5.1 COMSOL model for SW2 showing the generated mesh . . . . . . . . 95

5.2 SIMION CFA configuration from the side (normal to the $x-y$ plane). Electrons cycloid from right to left in this model. . . . . . . . . . . . . 96

5.3 Vsim Geometry with electrons. The RF wave is input on the edge of the domain, within the coaxial port. The RF wave travels within the dielectric region between the ground plane and the green meander line. Electrons are emitted from the cathode region, and cycloid right due to the crossed electric and magnetic fields. The electrons interact with the RF wave and give up their energy to amplify the RF wave. . . . . . 97 
5.4 View of the dimensions of the slow wave circuit and ports from the top view, normal to the $y$-axis. The green meander line comes down (in the $y$-direction) through the outer conductor of the coaxial cable, and then meanders above the dielectric (not shown) and ground plane shown in red on the $x-z$ plane. . . . . . . . . . . . . . . 101

5.5 View of the VSim model, showing the dimensions of the slow wave circuit and ports from the side view, normal to the Z-axis for the NU CFA study. . . . . . . . . . . . . . . . . . . . . . . 102

5.6 Top down view (normal to $y$-axis) of (a) the meander line geometry with a good alignment with the grid and (b) the corresponding $E_{y}$ field of a generic run. The green section denotes locations where $E_{y}=0$ which corresponds to conductor, and the blue part is vacuum. .......104

5.7 Top down view (normal to $y$-axis) of the meander line geometry with a poor alignment with the grid (a) and the corresponding $E_{y}$ field of a generic run (b). The green section denotes locations where $E_{y}=0$ which corresponds to conductor, and the blue part is vacuum. . . . . 105

5.8 Top down view (normal to $y$-axis) of the input coaxial cable geometry (a) and the corresponding $E_{y}$ field of a generic run (b). The green section denotes locations where $E_{y}=0$ which corresponds to conductor, and the blue part is vacuum. 106 
5.9 The boundary conditions used to control the electron beam injection and cycloid trajectory. The electrons are emitted at a potential $200 \mathrm{~V}$ more positive than the sole so that the cycloiding electrons do not easily collect on the sole. The beam electrode is placed there to control the beam injection into the region between the anode and sole. The end hats are outlined with a dotted line and are at $z=0$ and the upper edge of the $z$ domain. Periodic boundaries are at the edges of the $x$ and $z$ domain. The periodic BCs allow for smaller model by keeping smooth electric fields at the edges. . . . . . . . . . . . . . . . . 109

5.10 Corresponding potentials of the beam optics in the VSim model. . . . . 109

5.11 View of the dimensions model of the NU CFA with particles from the top view, normal to the $y$-axis. Electrons are shown in blue dots to demonstrate space charge spreading the beam towards the $z$ edges and to show the end hats reflecting the beam back towards the center. . . 110

5.12 Vsim particle boundary conditions showing the the dielectric and beam electrode, which are not particle sinks, and boundary absorber, cathode, sole, and end collector, which are particle sinks. . . . . . . . . 113

5.13 Vsim non-uniform mesh on the $\mathrm{X}-\mathrm{Z}$ plane. The green section is the meander line, red is the ground plane, The white circles are the space between the inner and outer conductor of the coaxial cable, and the black lines are the mesh. In X, regions which coincide with the circuit is 2 cells wide and regions between the circuit is 2 cells wide. In Z, the circuit region is 2 cells wide, but in the center, the length of the cells is increased. . . . . . . . . . . . . . . . . . . . . . . . . . . . . 119 
5.14 Vsim non-uniform mesh on the Y-X plane. The green section is the meander line, red is the ground plane, The white circles are the space between the inner and outer conductor of the coaxial cable, and the black lines are the mesh. In X, regions which coincide with the circuit is 2 cells wide and regions between the circuit is 2 cells wide. In Z, the circuit region is 2 cells wide, but in the center, the length of the cells is increased. . . . . . . . . . . . . . . . . . . . . . . . 120

5.15 E-field diagnostic showing the charge accumulation in the non-uniform grid model.

5.16 Cathode/Sole approximation one. The $E_{R F}=0$ region is placed right above the sole electrode so that electrons can be emitted into the interaction region from a potential less negative than the sole. The $E_{R F}=0$ sets the electric fields equal to zero to prevent accumulation of charge at the electron emission location. . . . . . . . . . . . . . 125

5.17 The first configuration of the cathode/sole approximation 2. Cathode potentials are pink, and sole potentials are green. Only the first three cathode potentials emit electrons. Electrons in this case have an 'erratic' trajectory as they leave the cathode. Also, the cycloid radius is a multiple of the cathode separation length, and many electrons just squeeze right back through the cathode potential at the right two cathode potential locations and are collected on the cathode/sole

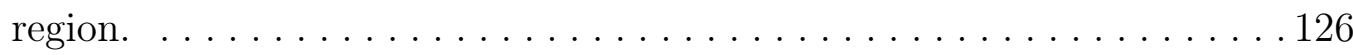

5.18 A close up on the cathode/region of cathode approximation two, showing the dimensions. This view also shows the 'erratic' electron trajectories as they leave the cathode . . . . . . . . . . . . . . . 127 
5.19 The second configuration of the Cathode/Sole approximation 2. Electron emission points are offset to the right of the cathode potentials (pink) in between the cathode and the sole. The cathode separation is optimized so that cycloid radius is offset from cathode segments down the tube and electrons can be repelled back into the interaction region. 128

5.20 A simple example of the divergence free region current propagation from the injection point to the domain edge at (a) $t=t_{0}$, (b) $t=t_{1}$, (c) $t=t_{2}$. Red dots indicate cells where the divergence is not equal to zero, and green dots are divergence free points. Index notation is used where $\mathbf{J}_{i j}$ indicates the current density at cell number $i$ in the $x$-direction. The divergence free region in this example is 3 cells high, and takes 3 timesteps for the current originating from the third row of cells to propagate to the $y=0$ edge. $\ldots \ldots \ldots \ldots \ldots \ldots \ldots \ldots \ldots \ldots$

5.21 The $y$-component of the electric field overlaid with cycloiding electrons in a (a) standard vacuum region and in a (b) divergence free region. The electron beam spread at $x=7.0 \mathrm{~cm}$ is shown in the figures to emphasize the difference in electron beam trajectories. . . . . . . . . . . 134

5.22 The y-component of the electric field overlaid with electrons electrons in (a) a standard vacuum region and (b) in a divergence free region . . 136

5.23 The x-component of the electric field overlaid with electrons (a) in a standard vacuum region and (b) in a divergence free region . . . . . . 137

5.24 Three spatial profiles: linear profile with positive slope and no DC current in blue, linear profiles with negative slope and 50\% DC current in magenta, and a uniform profile in green. 
5.25 The sine wave electron emission profile compared to the $E_{R F x}$ field with $\phi_{\text {offset }}=0 \mathrm{rad}$ at $\omega t=\phi_{t}$. In this case the profile peaks are in the accelerating regions of the RF wave (out of phase).

5.26 The square pulse electron emission profile compared to the RF electric field in the $x$-direction $\left(E_{R F x}\right)$ at $\omega t=\phi_{t}$. In this case the pulse is "out of phase" with the RF field. . . . . . . . . . . . . . . . . . . . 142

5.27 Proposed resonant circuit to minimize consumed power by the GFEA. 144

5.28 Calculated electron current density pulses from the MIT GFEAs [25] for a sinusoidal input from (a) $V_{g}=20-50 \mathrm{~V}$ and (b) $V_{g}=35-50 \mathrm{~V} .145$

6.1 S-parameters of SW2 from both simulation (COMSOL) and measured (network analyzer) showing the cutoff frequency. . . . . . . . . . . . 148

6.2 Measured electric field intensity for SW2 for frequencies (a) $500 \mathrm{MHz}$ and (b) $1.15 \mathrm{GHz} \ldots \ldots \ldots \ldots \ldots$. . . . . . . . . . . . . . . . . . . . . . . . . . . .

6.3 COMSOL simulated electric field intensity for SW2 for frequencies (a)

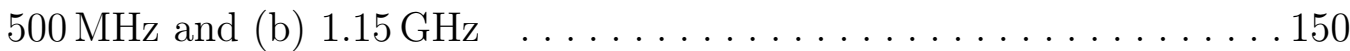

6.4 2D spatial FFT of the measured and simulated electric field intensity for SW2 for frequencies (a) $500 \mathrm{MHz}$ and (b) $1.15 \mathrm{GHz} \ldots \ldots \ldots \ldots 151$

6.5 (a) Voltage through time of the VSim dispersion model using an impulse signal and periodic boundaries in $x$, and (b) the FFT of that signal.

6.6 Measured and Simulated dispersion diagram for SW2 using (a) $x$ component of the spatial FFT, and using (b) the FFT along the center in the $x$-direction.

xxiv 
6.7 Measured and simulated retardations of SW2 using the FFT along the

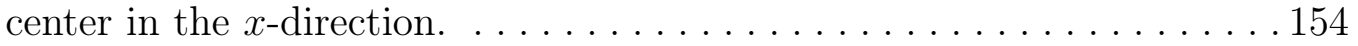

6.8 Experimental I-B Curves . . . . . . . . . . . . . . . . . . . 160

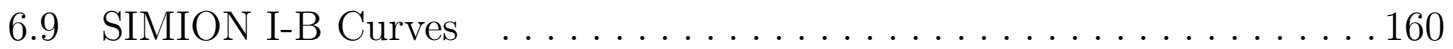

6.10 Sample Trajectories for three different magnetic fields. With lower magnetic fields, more electron trajectories collect on the slow wave circuit, at high magnetic fields, most of the current travels down the

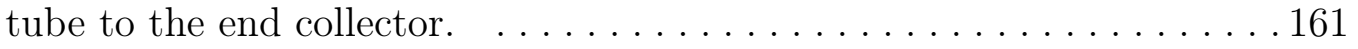

6.11 The SW2 model used in VSim with electrons shown in blue and the slow wave circuit in red . . . . . . . . . . . . . . . . . . 164

6.12 Gain along the length of the SW2 circuit compared to the predicted gain of Pierce theory for $P_{r f}=1 \mathrm{~W}, I_{\text {beam }}=20 \mathrm{~mA}$ and $150 \mathrm{~mA}$, and a circuit whose length is 2.5 times as long as the experimental circuit. 165

7.1 (a)Dispersion and (b) the retardation vs. frequency of SW3 as calculated from the VSim model.

7.2 Vsim model showing the dummy electrode used to control the beam injection in the NU CFA and the cathode placement dimensions. . . . .171

7.3 (a) Gain and (b) the measured currents on various electrodes vs. the position of the cathode for $I_{\text {beam }}=150 \mathrm{~mA}, B=5.2 \mathrm{mT}$, and $V_{a s}=$ $1250 \mathrm{~V}$. The Position at $0 \mathrm{~m}$ corresponds to directly underneath the first period of the slow wave circuit.

7.4 Electron trajectories corresponding to (a) $1.3 \mathrm{~cm}$ cathode offset placement and (b) $4.0 \mathrm{~cm}$ cathode offset placement. Electrons are shown in blue and the slow wave circuit is red. 
7.5 (a) Gain and (b) the measured currents on various electrodes vs. the potential of the beam electrode for $I_{\text {beam }}=150 \mathrm{~mA}, B=5.2 \mathrm{mT}$, and $V_{a s}=1250 \mathrm{~V}$. The Position at $0 \mathrm{~m}$ corresponds to directly underneath the first period of the slow wave circuit. $\ldots \ldots \ldots \ldots \ldots \ldots \ldots \ldots$

7.6 Electron trajectories for $V_{A S}=1550 \mathrm{~V}$, and different values of $V_{C S}$, (a) $200 \mathrm{~V}$, (b) $300 \mathrm{~V}$, (c) $400 \mathrm{~V}$, and (d) $500 \mathrm{~V} \ldots \ldots \ldots \ldots \ldots \ldots \ldots$

7.7 (a) Gain vs. $V_{c s}$ for various $V_{a s}$ and (b) Gain vs. $V_{a s}$ for various $V_{c s}$. The retardation is held constant by adjusting the magnetic field to keep the $V / B$ ratio constant.

7.8 (a) Gain vs. Magnetic Field for $I_{\text {beam }}=150 \mathrm{~mA}, V_{a s}=1250 \mathrm{~V}, V_{b o}=$ $-200 \mathrm{~V}$ and (b) the corresponding currents from VSim. ......... 180

7.9 (a) Gain vs. injected beam current and comparison to experimental data found in Browning et al. $[14,15]$ and (b) the corresponding currents from VSim.

7.10 Efficiency vs. injected beam current and comparison to experimental data found in Browning et al. $[14,15] \ldots \ldots \ldots \ldots \ldots \ldots \ldots \ldots$

7.11 (a) Gain vs. Frequency and comparison to experimental data found in Browning et al. [14] and (b) the corresponding currents............ 185

7.12 (a) Gain vs. RF power for $I_{\text {beam }}=150 \mathrm{~mA}$ and (b) the corresponding efficiency vs. RF input power. . . . . . . . . . . . . . . . . . . . 191

7.13 Signal-to-Noise ratio for different powers $\ldots \ldots \ldots \ldots \ldots \ldots \ldots \ldots$

7.14 The input and output voltage with $I_{\text {beam }}=150 \mathrm{~mA}$ for (a) $P_{r f}=$

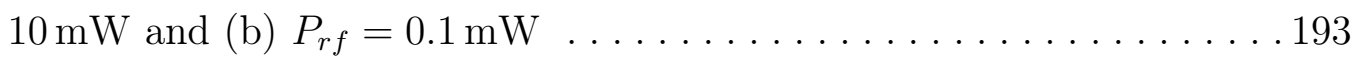

7.15 Gain vs. RF power for $I_{\text {beam }}=100 \mathrm{~mA} \ldots \ldots \ldots \ldots \ldots \ldots \ldots$ 
7.16 (a) The Gain vs. the SW circuit length with $P_{r f}=1 \mathrm{~W}$ and $I_{\text {beam }}=$ $150 \mathrm{~mA}$ and (b) the corresponding simulated efficiency .......... 197

7.17 (a) The Gain vs. the SW circuit length with $P_{r f}=10 \mathrm{~W}$ and $I_{\text {beam }}=$ $150 \mathrm{~mA}$ and (b) the corresponding simulated efficiency ......... 198

7.18 Gain along the length of the circuit for both VSim simulation (red), and Pierce theory (blue). . . . . . . . . . . . . . . . . . 199

7.19 The AC coupled RMS $y$-component of the electric field, in red, and the moving average, in blue, along the length of the circuit for (a) $0.625 \mathrm{~cm}$,

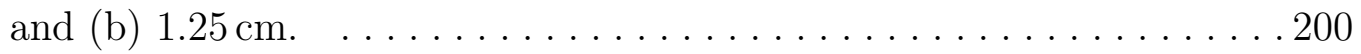

7.20 Two electron trajectories with different cycloid radii. The blue trajectory has half the cycloid radius than the red trajectory. $E_{r 1}$ and $E_{r 2}$ are the estimated energy extracted from the smaller and larger radii, respectively. ................................203

7.21 A variation of the cathode approximation method one to test the $E_{R F}=0$ region effects on gain. This variation emits electrons similarly to the injected beam configuration but there is the $E_{R F}=0$ region. Electrons are allowed to enter the region but no RF fields are calculated there. ...................................206

7.22 (a) The gain and the corresponding (b) currents vs. the conducting

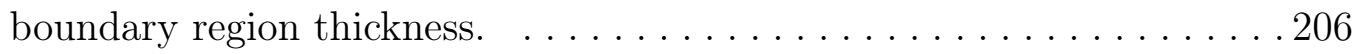

7.23 Electron trajectories for 7 emitters, $V_{A S}=1650 \mathrm{~V}, B=6.8 \mathrm{mT}$, and different values of $V_{c s}$, (a) $200 \mathrm{~V}$, (b) $300 \mathrm{~V}$, and (c) $400 \mathrm{~V}$. .......209

7.24 (a) The gain and the corresponding (b) currents vs. the cathode separation for $V_{a s}=1650 \mathrm{~V}$ and $B=6.7 \mathrm{mT}$. ..............211 
7.25 (a) The gain and the corresponding (b) currents vs. the magnetic field with $N_{c 2 c}=11$ cells for the cathode approximation $2 \ldots \ldots 212$

7.26 The two setups to determine the effect of the divergence free region. Emission from a (a) conducting region and from (b) the divergence-free region. . . . . . . . . . . . . . . . . . . . . . . . 213

7.27 Gain vs. emission length, $L_{e}$, for the both divergence-free (DF) cathode approximation and the raised emitting conductor. . . . . . . . . . 214

7.28 Optimization of the CFA parameters using the Divergence-free region showing the (a) gain and (b) currents vs. $V_{a s} . V_{c s}=200 \mathrm{~V}$ and the magnetic field is optimized for each voltage point to maximize gain. . . . 216

7.29 (a) The gain and the corresponding (b) SNR vs. the RF input power for the injected beam configuration wit a $1.5 \mathrm{~cm}$ emitter length and the distributed cathode configuration with $10 \mathrm{~cm}$ and $20 \mathrm{~cm}$ emitter

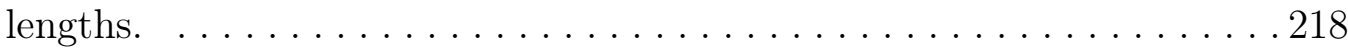

7.30 (a) The gain and the corresponding (b) SNR vs. the cathode length for the distributed cathode approximation with $P_{r f}=0.1 \mathrm{~W} \ldots \ldots 219$

7.31 (a) The gain and the corresponding (b) currents vs. the beam current for the uniform emission profile with $L_{e}=20 \mathrm{~cm}$ and $P_{r f}=0.1 \mathrm{~W}$. . . 220

7.32 (a) The gain and the corresponding (b) SNR vs. the phase difference between the beam profile and the RF wave for $L_{e}=30 \mathrm{~cm}, P_{r f}=1 \mathrm{~W}$, and $I_{\text {beam }}=150 \mathrm{~mA}$.

7.33 (a) The gain and the corresponding (b) SNR vs. the phase difference between the beam profile and the RF wave for $L_{e}=30 \mathrm{~cm}, P_{r f}=1 \mathrm{~W}$, and $I_{\text {beam }}=150 \mathrm{~mA}$. .225 
7.34 (a) The gain and the corresponding (b) SNR vs. the phase difference between the beam profile and the RF wave for $L_{e}=30 \mathrm{~cm}, P_{r f}=1 \mathrm{~W}$,

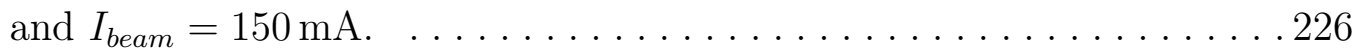

7.35 The (a) phase and the (b) gain along the circuit for the square pulse profile with $\phi_{\text {offset }}=0 \mathrm{rad}, L_{e}=30 \mathrm{~cm}, L_{p}=1 \mathrm{~cm}, P_{r f}=1 \mathrm{~W}$, and

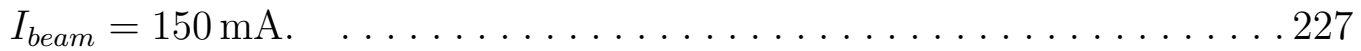

7.36 The (a) phase and the (b) gain along the circuit for the square pulse profile with $\phi_{\text {offset }}=\pi \mathrm{rad}, L_{e}=30 \mathrm{~cm}, L_{p}=1 \mathrm{~cm}, P_{r f}=1 \mathrm{~W}$, and $I_{\text {beam }}=150 \mathrm{~mA}$.

7.37 (a) The gain and the corresponding (b) SNR vs. the phase difference between the beam profile and the RF wave for $L_{e}=30 \mathrm{~cm}, P_{r f}=1 \mathrm{~W}$,

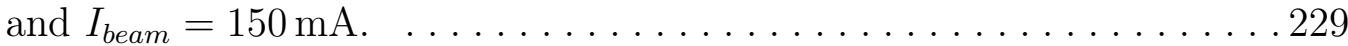

7.38 (a) The gain and the corresponding (b) output power vs. the RF input power on the circuit for both the modulated current and the injected beam with $I_{\text {beam }}=150 \mathrm{~mA}$. The modulated cathode uses $L_{e}=30 \mathrm{~cm}$. The RF input power on the $\mathrm{x}$-axis does not include the modulated cathode power. In (a), the red line includes a $1 \mathrm{~W}$ modulated cathode power and the blue line includes a $0.1 \mathrm{~W}$ modulated cathode power. . . 230

7.39 (a) The efficiency and the (b) SNR vs. the RF input power on the circuit for both the modulated cathode in red and the injected beam in green with $I_{\text {beam }}=150 \mathrm{~mA}$. The modulated cathode uses $L_{e}=30 \mathrm{~cm}$. The RF input power on the $\mathrm{x}$-axis does not include the modulated cathode power. 
7.40 The RF input signal contribution to (a) gain and the corresponding (b) output power vs. the RF input power on the circuit for both the modulated and injected beam cathode. The RF input power on the $\mathrm{x}$-axis does not include the modulated cathode power.

7.41 (a) The gain and the corresponding (b) output power vs. the beam current for both the modulated and uniform current distributions with $P_{r f}=0.1 \mathrm{~W}$. In $(\mathrm{a})$, the red line includes a $1 \mathrm{~W}$ modulated cathode power, the blue line includes a $0.1 \mathrm{~W}$ modulated cathode power, and the green line shows the uniform current case. The modulated cathode uses $L_{e}=30 \mathrm{~cm}$ and the uniform current uses $L_{e}=20 \mathrm{~cm} . \ldots \ldots 233$

7.42 (a) The efficiency and the (b) SNR vs. beam current on the circuit for the modulated distribution in red and the uniform distribution in

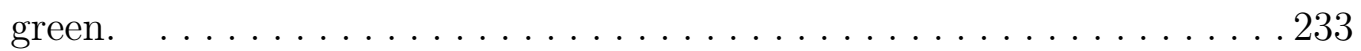

7.43 (a) The currents for the modulated cathode compared to (b) the currents of the uniform cathode for the beam current sweep.

7.44 Electron trajectories for the square pulse emission profile for $V_{a s}=$

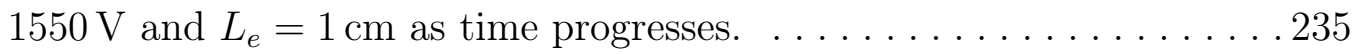

7.45 Electron trajectories for various beam injection types and RF input powers with $V_{a s}=1550 \mathrm{~V}$ and $I_{\text {beam }}=150 \mathrm{~mA} \ldots \ldots \ldots \ldots \ldots$

7.46 Diagram of the shielded cathode slit concept. Lateral gated field emitters on each side of the slit emit electrons and are pushed out through the slit in the sole electrode by the pusher electrode [112]. . . 247 
7.47 Diagram of a CFA using end hat assisted injection. The majority of the electron current is supplied by a traditional thermionic or secondary emitting cathode (violet arrows), and modulated electrons are injected in at the end hats (blue arrows). . . . . . . . . . . . . . . 248

7.48 Diagram of the GFEA assisted secondary emitting cathode. GFEA locations (these could be hop funnel or the shielded cathode slits) emit electrons in phase with the RF wave with energies so that they collide with the secondary emitting sole at the optimum energy $\left(E_{\max }\right)$ to emit the maximum amount of secondary electrons $\left(\delta_{\max }\right) \ldots \ldots \ldots \ldots$

A.1 Measurement setup schematic. There are two potentials at which signals are measured, earth ground and CFA ground. Recording earth ground measurements is easily done by LabVIEW data acquisition (DAQ) Crate. CFA ground based measurements transmit the measurement signal through analog opto-isolaters. Control of CFA ground based currents and voltages is done by a CFA ground based microcontroller which communicates to the earth ground computer through

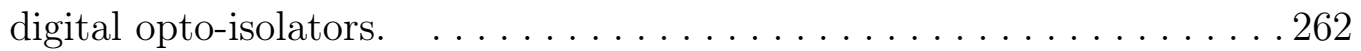

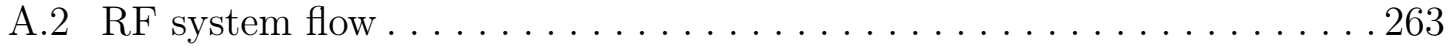

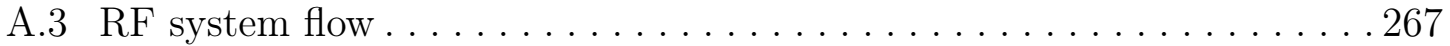

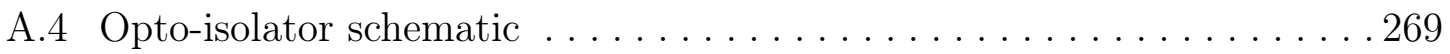




\section{LIST OF ABBREVIATIONS}

MVED - Microwave Vacuum Electron Device

FEA - Field Emitter Array

GFEA - Gated Field Emitter Array

pFED - Printable Field Emission Display

SEM - Scanning Electron Microscopy

SEY - Secondary Electron Yield

UHV - Ultra High Vacuum

I-V curve - Transmitted Current Vs. Hop Voltage

CFA - Crossed-Field Amplifier

NU CFA - Northeastern University Crossed-Field Amplifier

BSU CFA - Boise State University Crossed-Field Amplifier

RF - Radio Frequency (commonly used to describe electromagnetic waves in general)

CFA1, CFA2 - Refers to the two Crossed-Field Amplifiers created at Boise State University which use the slow wave circuits SW1 and SW2 respectively.

SW1, SW2 - The first and second slow wave circuits designed at BSU

TWT - Traveling Wave Tube

MIT - Massachusetts Institute of Technology 
PIC - Particle In Cell

MUMPS - Multifrontal Massively Parallel Sparse direct solver

$3 \mathrm{D}$ - Three Dimensional

FDTD - Finite Difference Time Domain

PEC - Perfect Electric Conductor

CFL - Courant-Friedrichs-Lewy

NU - Northeastern University

Sim. - Simulation

Exp. - Experiment

SNR - Signal-To-Noise Ratio

LTCC - Low Temperature Co-Fired Ceramic

BSU - Boise State University

FFT - Fast Fourier Transform

PML - Perfectly Matched Layer

ES - electrostatic

BC - Boundary Condition

EM - Electromagnetic

DC - Direct Current (commonly used to specify a constant voltage, ie. DC bias voltage)

SW - Slow Wave

HV - High Voltage 
RMS - Root Mean Squared

PSD - Power Spectral Density 


\section{CHAPTER 1}

\section{INTRODUCTION}

Microwave vacuum electron devices (MVEDs) are devices that utilize free electrons in vacuum to interact with the geometry, electric and magnetic fields, and radio frequency $(\mathrm{RF})$ waves to create an amplifier or an oscillator. There are many different MVED device configurations, but these generally fall under three categories: O-Type (linear), M-Type (crossed-field), and fast-wave devices . Linear devices are configurations where the electron is focused by a magnetic field aligned with the beam. These type of devices extract kinetic energy from the beam to produce gain or oscillations. Examples of these devices are gridded tubes [1-5], klystrons [1, 2, 4, 6] and traveling wave tubes (TWTs) [1-4, 7-10]. Crossed-field devices are configurations with a static electric field perpendicular to a static magnetic field, and the electrons follow a cycloidal trajectory with the average drift velocity in the direction perpendicular to both fields. These devices extract potential energy from the beam to get gain. Examples of these are Magnetrons [1-4, 11-13] and Crossed-Field amplifiers (CFAs) [1-4, 13-19]. Fast-wave devices use the cyclotron frequency for operation; a gyrotron $[1-4,20]$ is one example of many gyro devices.

MVEDs fall into a niche of high power and/or high frequency devices. Solid-state devices have not yet achieved the power density capabilities of MVEDs. Figure 1.1 shows a chart comparing MVEDs with solid state devices [2]. Each MVED 
configuration has its own advantages and disadvantages with regards to several figures of merit: bandwidth, gain, maximum power, efficiency, size, and signal to noise ratio. A summary of the advantages and disadvantages of each device is difficult to compile because of the diversity within each group and the application. An attempt is made here, and Table 1.1 summarizes the advantages and the applications of common MVEDs. Generally, all the figures of merit listed for the device type cannot all be maximized simultaneously for one particular device because interconnectivity of the figures of merit. For example, generally gain is inversely proportional to the bandwidth, so the maximum listed gain listed for that device cannot exist simultaneously with the maximum bandwidth. Also, power at higher frequencies is much lower than power at lower frequencies, so the maximum listed power is generally not associated with the maximum listed frequency. Therefore, the specifications listed in the chart are rather subjective.

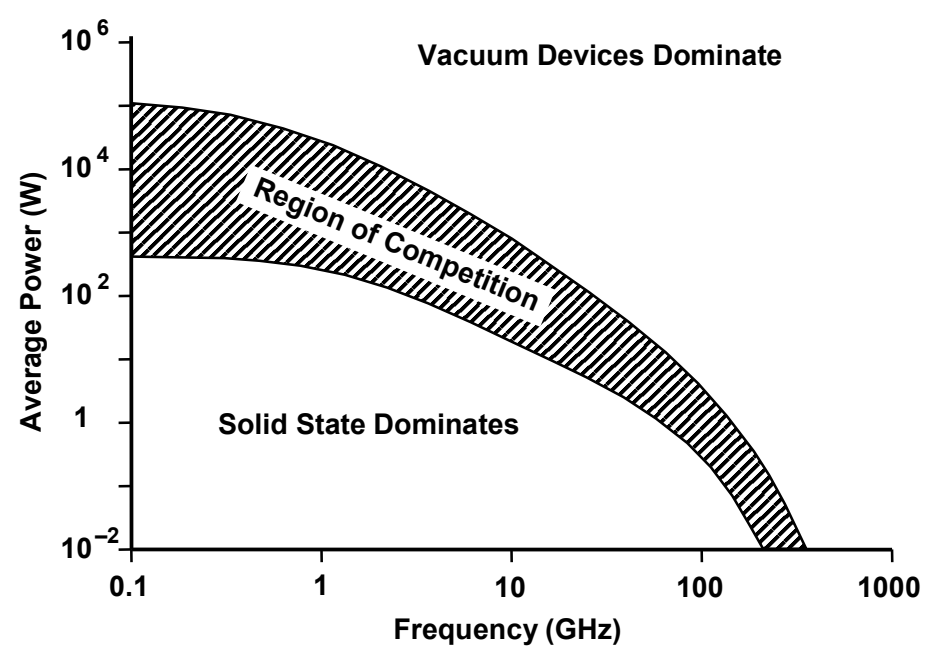

Figure 1.1: Range of applications of MVEDs in comparison with solid state devices. Reproduced with permission from [2]. 
Table 1.1: Summary of Microwave Vacuum Electron Devices

\begin{tabular}{|c|c|c|}
\hline Device & Figures of Merit & Applications \\
\hline $\begin{array}{l}\text { Gridded } \\
\text { Tubes }\end{array}$ & $\begin{array}{l}\text { - Low frequencies } \\
\text { - Low power } \\
\text { - Low cost } \\
\text { - Compact size }\end{array}$ & \\
\hline Klystrons & $\begin{array}{l}\text { - High Gain }(>60 \mathrm{~dB}) \text {, } \\
\text { - Low Bandwidth at low } \\
\text { powers }(<5 \%) \\
\text { - } \text { Moderate bandwidth at } \\
\text { high powers }(10-15 \%) \\
\text { - } \text { High Power }(>100 \mathrm{MW} \\
\text { pulsed, >1 MW CW }) \\
\text { - Frequency Range } \\
(\approx 1-100 \mathrm{GHz}) \\
\text { - Large size }\end{array}$ & $\begin{array}{l}\text { - Particle accelerators } \\
\text { - Deep space } \\
\text { communications } \\
\text { - TV broadcasting } \\
\text { - Medical imaging [21] }\end{array}$ \\
\hline TWTs & $\begin{array}{l}\text { - } \text { Moderate to High Gain } \\
(30-50 \mathrm{~dB}), \\
\text { - } \text { High Bandwidth } \\
(2 \text { octaves }) \text { at low powers } \\
(<200 \mathrm{~kW}) \\
\text { - } \text { Moderate bandwidth } \\
(<15 \%) \text { at high powers } \\
\text { - } \text { Low to moderate power } \\
\text { ( } 1 \text { MW CW }) \\
\text { - Frequency }(<100 \mathrm{GHz})\end{array}$ & $\begin{array}{l}\text { - Radar } \\
\text { - Satellite communications }\end{array}$ \\
\hline CFAs & $\begin{array}{l}\text { - Low Gain }(<20 \mathrm{~dB}) \text {, } \\
\text { - High power }(>10 \mathrm{MW} \\
\text { pulsed, }>400 \mathrm{~kW} \mathrm{CW}) \text {, } \\
\text { - High efficiency }(\lesssim 70 \%) \text {, } \\
\text { - Frequency Range } \\
(<20 \mathrm{GHz}) \\
\text { - Moderate bandwidth } \\
\text { - }(<15 \%) \\
\text { - Compact size }\end{array}$ & $\begin{array}{l}\text { - Radar } \\
\text { - Electronic Counter } \\
\text { Measures } \\
\text { - Noise Generators } \\
\text { - Particle Accelerators }\end{array}$ \\
\hline
\end{tabular}




\begin{tabular}{|c|c|c|}
\hline Device & Figures of Merit & Applications \\
\hline Magnetrons & $\begin{array}{l}\text { - Low bandwidth, } \\
\text { - High Power }(<1 \mathrm{GW} \\
\text { pulsed, }<100 \mathrm{~kW} \mathrm{CW}) \text {, } \\
\text { - High Efficiency (up to } \\
\text { 90\%) }\end{array}$ & $\begin{array}{l}\text { - Microwave Ovens } \\
\text { - Radar } \\
\text { - Medical imaging [21] } \\
\text { - Particle accelerators [22] }\end{array}$ \\
\hline Gyrotrons & $\begin{array}{l}\text { - High power }(<2 \mathrm{MW} \\
\text { CW pulsed, }>400 \mathrm{~kW} \\
\text { CW }) \text {, } \\
\text { - } \text { Moderate efficiency } \\
(<50 \%), \\
\text { - High frequency } \\
(10 \mathrm{GHz}-1 \mathrm{THz}) \\
\text { - } \text { Compact size }\end{array}$ & $\begin{array}{l}\text { - Nuclear Fusion } \\
\text { - Radar } \\
\text { - Medical imaging }\end{array}$ \\
\hline
\end{tabular}

No MVED meets the needs of all applications, and each choice has compromises. The focus of the dissertation is on crossed-field amplifiers, and the most notable features of these devices are the high power capability with decent bandwidth in a compact size. The klystron outperforms the CFA in terms of gain and maximum power, but the device is very large at lower frequencies. So for more portable applications, the CFA is more practical. The disadvantages of CFAs are the low gain and relatively high noise, which limits the CFA in several applications. Improving the gain and noise characteristics would make the CFA much more appealing for a variety of applications, and this is the ultimate goal of this dissertation.

\section{$1.1 \quad$ Device Concept}

The goal of this research was to demonstrate a linear format CFA which uses gated field emitter arrays (GFEAs) [23-27] as the electron source to spatially and temporally vary the injected electron current density in order to maximize efficiency, gain, and 
bandwidth and to minimize noise. Linear format in this case is not to be confused with O-type devices where the beam and the magnetic field are in the same direction, but refers to the linear geometry. The original goal of this research included an experimental component, but the CFA experiments showed no gain. Some experimental components are still presented here, but the focus is primarily on simulation.

Current crossed-field devices use thermionic or secondary electron emitting cathodes, with the exception of some magnetrons [28, 29] and the A6 magnetron which uses a transparent cathode $[11,12]$ where explosive emitters $[30,31]$ are used. In the transparent cathode work, current is emitted from explosive emitters at discrete locations in the geometry, but each source has the same approximate current. That work showed an increase in magnetron oscillator performance as discussed later. In this dissertation, the cathode will emit from discrete GFEA sources but with varying currents, in an amplifying configuration. By spatially varying the currents in the interaction region, better performance with respect to efficiency and gain is expected.

Gated field emitters are much more efficient electron current sources than typical thermionic cathodes [2, 24, 32]; they have higher modulation frequency capability, and they have the advantage of easy spatial control. The use of GFEAs in MVEDs is not a new concept $[8,9,11,12,33]$, but in general, it has not been implemented due to emission current and reliability constraints. In this work, a technique of using hop funnels [34-38] to integrate GFEAs into a MVED device will be discussed [39], but this approach was not implemented due to experimental problems as explained later.

Three different simulation softwares were used in this work: SIMION [40], COMSOL [41], and VSim [42]. The simulations modeled three different CFAs in total: two variations were designed and built at Boise State University (BSU) and one was built and studied at Northeastern University (NU) [14, 15]. The electron trajectories 
were modeled in SIMION and VSim and were compared with experimental results of the BSU CFAs. The slow wave circuit dispersion characteristics were modeled in COMSOL and VSim and were compared with the experimental BSU CFAs. The full CFA simulation was modeled in VSim, but because the BSU CFAs produced no gain, the simulation results were compared with experimental measurements of a very similar $\mathrm{CFA}$ used at $\mathrm{NU}$ in order to validate the simulation model. Each of these CFAs use the same basic design, but with different dimensions. The same basic VSim model can be used to simulate each design.

The simulation results confirmed the poor performance of the BSU CFAs, and the focus of the work was shifted to the NU CFA design. An in depth study of the general CFA operation and physics was performed and was confirmed with theory and

the results of the NU work. A variation of the NU model, the spatial modulation is performed using a distributed cathode with spatial- and time-varying electron current distributions, was developed to study the effects on gain, signal to noise ratio (SNR), and efficiency.

\subsection{Research Objectives and Contributions}

Current CFAs demonstrate high power capability with good bandwidth in a compact size, but they have low gain and relatively high noise. To extend their usefulness, a considerable amount of effort has been put forth to improve gain and the noise characteristics of CFAs. The objective of this research is to see if it is possible to use a spatially and temporally modulatable cathode in place of traditional secondary emitting and thermionic cathodes to improve gain and lower noise in CFAs.

The focus of this dissertation is mainly on simulation work. One of the original 
goals was to perform original GFEA experimental work, but because of experimental problems, the experimental work was only used to validate the simulation model. The primary contributions of this research are:

1. Development of a linear format CFA simulation model in VSim [42] which implements a distributed cathode.

2. Comparison of different static distributed electron emission profiles.

3. Demonstration via simulation of improved performance using a temporally modulated electron emission profile. This is the main contribution to CFA research. The small-signal-gain and the signal-to-noise ratio of the device dramatically improves using this modulated cathode implementation and has the potential to increase the maximum output power of the device by improving mode-locking.

The first phase of the research validates the VSim simulation model against theory, experiments, and simulation results from COMSOL and SIMION. Experiments determining the slow wave circuit behavior and electron beam trajectory of the BSU CFA were performed at BSU and compared against the simulations. The full CFA operation simulation using VSim was compared against experimental results performed at NU on the NU CFA. These results are also compared with and confirm the current observations in CFA literature.

The next phase of the research studied the effects of different static electron emission profiles from a distributed cathode. These electron current distributions were studied with respect to gain, SNR, and efficiency. There are many published research efforts which use many different cathode types and implementation, but currently there is no published study comparing different electron emission profiles in the same device. 
The third phase of the research studied the effects of different modulated electron emission profiles. Both an injected and distributed beam were studied with respect to gain, SNR, bandwidth, and efficiency. Currently there are no publications which utilize a modulated electron beam in a CFA.

The last phase was to investigate the performance and the plausibility of this distributed cathode approach for real CFA applications. The model studied in this research is a low power device, and in order to achieve the goal of implementing a high power and high gain CFA, the modulated cathode must be achievable in a higher power device.

\subsection{Overview of the Dissertation}

Three different CFA designs are described here. All are of the same type but with different dimensions and operating frequencies. The first CFA designs were simulated and were tested via experiment. These designs showed no electron-RF wave interaction experimentally, and the results were corroborated via simulation. The research effort shifted to a design used in previous work at Northeastern University [14], which showed moderate gain $(7 \mathrm{~dB})$ in that work. The bulk of the contributions of this work use the NU CFA design and are performed via simulation using Vsim.

A general background of CFAs is presented in Chapter 2. There are two important components of the CFA: the slow wave structure and the electron beam. A general background on these two concepts and their function is given. A general overview of the numerical methods used in simulation software used in this research is also presented. Also, a detailed overview of the NU CFA design studied in this work is given. 
In Chapter 3, the proposed CFA and research objectives are explained in detail. The general research chronology is described, starting from the BSU CFA experimental and simulation work, to the NU CFA simulation work, and to the distributed cathode simulation work.

Chapter 4 outlines the experimental design for the BSU CFA. Three experiments were performed: one experiment to measure the slow wave circuit dispersion characteristics, another experiment to determine the electron beam trajectory characteristics, and one to test the full electron beam interaction with the RF wave.

Chapter 5 describes the SIMION, COMSOL, and VSim simulation models in detail. A small portion of the research was performed using SIMION and COMSOL, so only a brief overview of those simulations are given. The bulk of the research was performed with VSim, and a very thorough overview of that software and the models are provided. The injected beam VSim model variation, which is used to validate the VSim model against experiments, is discussed in great detail. The distributed beam VSim simulation models, which is the focus of this research, are also discussed in great detail. The spatially- and time-varying distributed cathode methods are presented here, and the approximation to implement the distributed cathode is explained.

Chapter 6 presents the results of the experiments (using the experimental setup described in chapter 4) and simulations (using the simulation models described in chapter 5) performed on the BSU CFA. The experimental results matched up with simulation rather well, and the simulation results confirm and determine the reason for the poor performance of the BSU CFA designs. This result justifies the switch of the CFA design to the one used at NU.

Chapter 7 presents the simulation results of the NU CFA design. Detailed studies of the device physics and of different methods to optimize gain are presented and 
compared with theory. These results are compared and validated against the NU experimental results. Finally, the contributing factor in this research, using a variation of the NU design, the different spatially- and time-varying distributed cathode results are finally presented and analyzed. The benefits of the distributed cathodes are clearly outlined, and a thorough discussion of the impact of these results is given.

Chapter 8 summarizes the notable results in these studies and draws conclusions. The benefits and costs of the distributed cathode studies are all outlined, and the possible applications are given. Details on the next step of this research are also provided. 


\section{CHAPTER 2}

\section{BACKGROUND}

\subsection{Crossed-Field Amplifiers}

There are several aspects in which CFAs can be grouped: mode of operation (forward or backward wave), electron beam source (injected beam or emitting sole), geometry (cylindrical or linear), and beam collection (reentrant beam or end collector). Figure 2.1 shows an example of a cylindrical, injected beam, backward wave, non-reentrant CFA [17]. The geometry is obviously cylindrical where the sole electrode is surrounded by the RF circuit which also acts as the anode. A static electric field is created by the potential difference between the sole and RF circuit. The electron beam is injected at one point, labeled cathode, thus classifying this as an injected beam. The grid and accelerating anode control the electron trajectories upon entering the interaction space. Electrons cycloid clockwise around the sole, interact with the RF wave which is traveling counter clockwise along the RF circuit, and are collected on the collector. The collector at the end of the interaction space prevents electrons from 'reentering' at the beginning of the interaction space; thus this is a non-reentrant device. The electron beam travels in the opposite direction of the group velocity of the RF wave which classifies this a backward wave device. The attenuator on the delay line is used to prevent amplification of other modes in the circuit. 


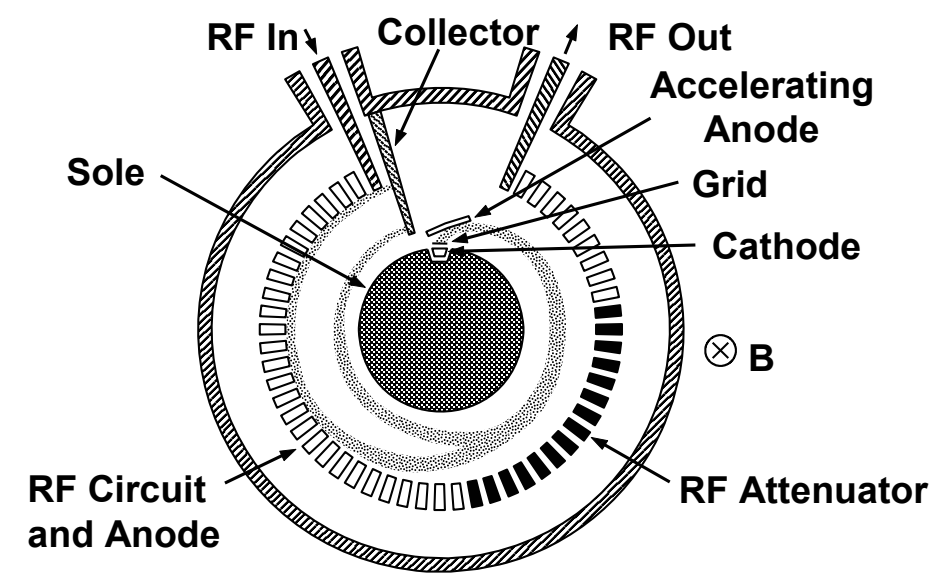

Figure 2.1: Cylindrical, injected beam, non-reentrant, backward wave crossed field amplifier. [17]

Figure 2.2 shows an example of a cylindrical, emitting sole, forward wave, reentrant CFA. In the emitting sole design, electrons are emitted from the entire center electrode, similar to the magnetron. The center electrode can be a thermionic cathode, a secondary emitting cathode (this is sometimes called a cold cathode), or a combination of both. Electrons travel clockwise and interact with the RF wave which is also traveling clockwise. Electrons that do no give up all their potential energy and collect on the RF circuit reenter the interaction region. These electrons are essentially 'recycled' and can give up any leftover energy from the previous rotation. This improves efficiency of the device over the end collector technique, but these electrons can cause feedback from output to the input. To minimize this feedback, a drift space is included to remove the modulation of the electron spokes. 


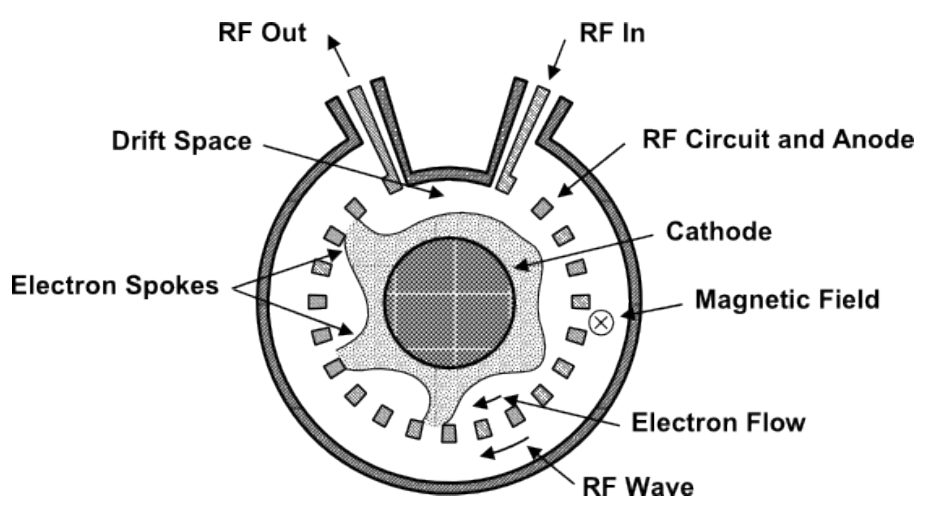

Figure 2.2: Cylindrical, emitting sole, reentrant, forward wave crossed field amplifier. [17]

Figure 2.3 shows a linear format, injected beam, forward wave CFA. Electrons are emitted from the cathode into the interaction region. The electrons travel down the tube and interact with the RF wave on the slow wave circuit, give up their potential energy, and collect on the delay structure. Electrons that do not collect on the delay line are collected by the end collector. Obviously, linear format CFAs are non-reentrant. This linear format design is very similar to the design used in this research. The first iteration in the research is very close to this injected format design. The desired final design will emit electrons from the sole and is explained in more detail later.

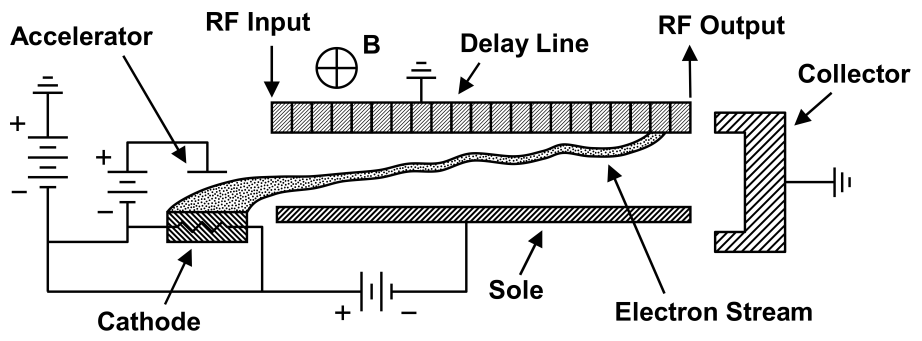

Figure 2.3: Linear format injected beam crossed field amplifier [2].

The linear format, non-reentrant CFAs are very similar to the cylindrical format non-reentrant designs. The mechanisms are the same for both geometries, but the 
main difference between the theory of these designs are that the governing equations for electron motion are either in cylindrical or Cartesian coordinates. Because the design in this research is of a linear format, the equations presented in this work are for the linear format.

All the CFA variations consist of two main parts: the slow wave circuit, also called delay line, and the electron beam. The slow wave circuit retards the phase velocity of the RF wave in order to easily interact with the electron beam. The electron beam travels close to the speed of the RF wave and forms "bunches" or "spokes", as they are called in cylindrical geometries, due to focusing from the RF wave. If the electron beam is slightly faster than the RF phase velocity, potential energy is extracted from the beam and provides amplification of the RF wave.

The slow wave structures are very dispersive, and an extensive overview on the subject is provided. First a discussion on wave velocities and dispersion and then a general overview of different slow wave circuits are presented. The motion of the electron beam is also very important. The cycloidal trajectory of the electron is discussed. Because field emitters and eventually hop funnels are proposed for our CFA, a brief overview of GFEAs and hop funnels is provided.

\subsection{Wave Velocities and Dispersion}

There are many ways to represent dispersion. A plot of phase velocity vs. frequency is the easiest to visualize and understand, but it shows limited information. Usually dispersion is represented as frequency vs wave number. This later view, although less intuitive, is much more informative and contains all the information about the slow wave circuit. This approach is discussed here. The phase velocity is represented by 
the ratio $\omega / \beta$, and the group velocity is the slope of the curve $\Delta \omega / \Delta \beta$, where $\omega$ is the angular frequency and $\beta$ is the wave number.

An example of the dispersion in simple rectangular conductive wave guide [2] is given in Fig. 2.4. Note that as the frequency approaches the cutoff frequency, $\omega_{c}$, the wave number becomes infinitely small. The wavelength becomes very large as the group velocity slows down, and propagation is cutoff. Below this frequency, there is no propagation. As the frequency increases, the slope of the line approaches the speed of light. It is also important to note that waves can travel in both directions with identical characteristics, so the diagram is symmetric about the $\omega$ axis.

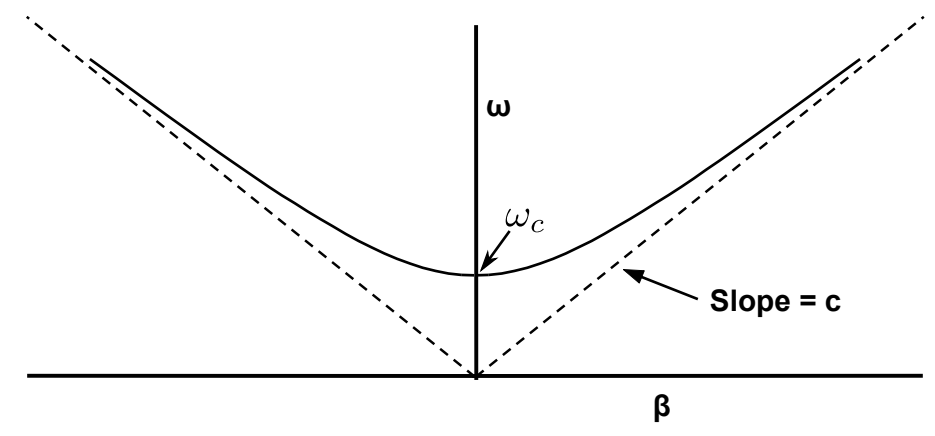

Figure 2.4: Dispersion diagram for waves traveling in either direction in a rectangular waveguide [2].

For periodic structures, there are frequency harmonics and spatial harmonics called Hartree harmonics [43]. Many different modes can exist on the circuit, and the modes are periodic with respect to the period of the device. A detailed description of this can be found in Gilmour [7], but a summation is given here. Because the dispersion is directly related to the periodicity of the device, the wave number is now represented with $\beta L$, where $L$ is the period of the structure. The dispersion diagram for a periodically metallic loaded waveguide is shown in Fig. 2.5. The periodic nature of the loaded waveguides causes periodic reflections. Sometimes these reflections add 
in phase, resulting in propagation, and sometimes they are out of phase, resulting in cutoff. In this cutoff case, when the half-wavelength becomes equal to the period of the structure, $\beta L=\pi$, the reflected waves add out of phase with the forward waves, and propagation stops. If the wave number is allowed to continue to increase, a backward wave mode is excited. The space in the diagram where $\beta>\pi$ are called the Hartree harmonics.

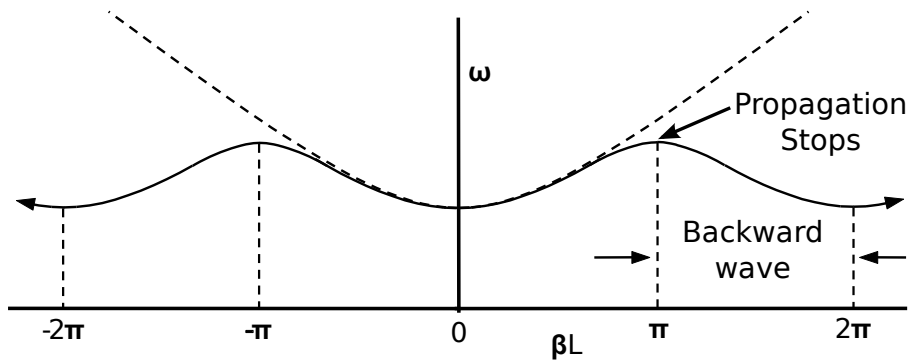

Figure 2.5: Dispersion diagram for waves traveling in either direction in a periodically loaded rectangular waveguide [2].

There are also harmonics in frequency as well. Fig 2.6 shows the dispersion diagram for the periodically loaded waveguide including the frequency harmonics. There are three regions highlighted in the figure to explain frequency effects. When applying a waveform from the source to the load in the positive direction in the periodically loaded waveguide, at a frequency below $\omega_{c 1}$, the group and phase velocity are both positive. This corresponds to region (a) in Fig. 2.6. As the frequency increases above $\omega_{c 1}$, propagation stops, which corresponds to the cutoff region. Increasing the frequency above $\omega_{2}$, the group velocity is still positive, but the phase velocity becomes negative. This corresponds to region (b) in Fig. 2.6. This backward mode exists until the second cutoff region occurs. 


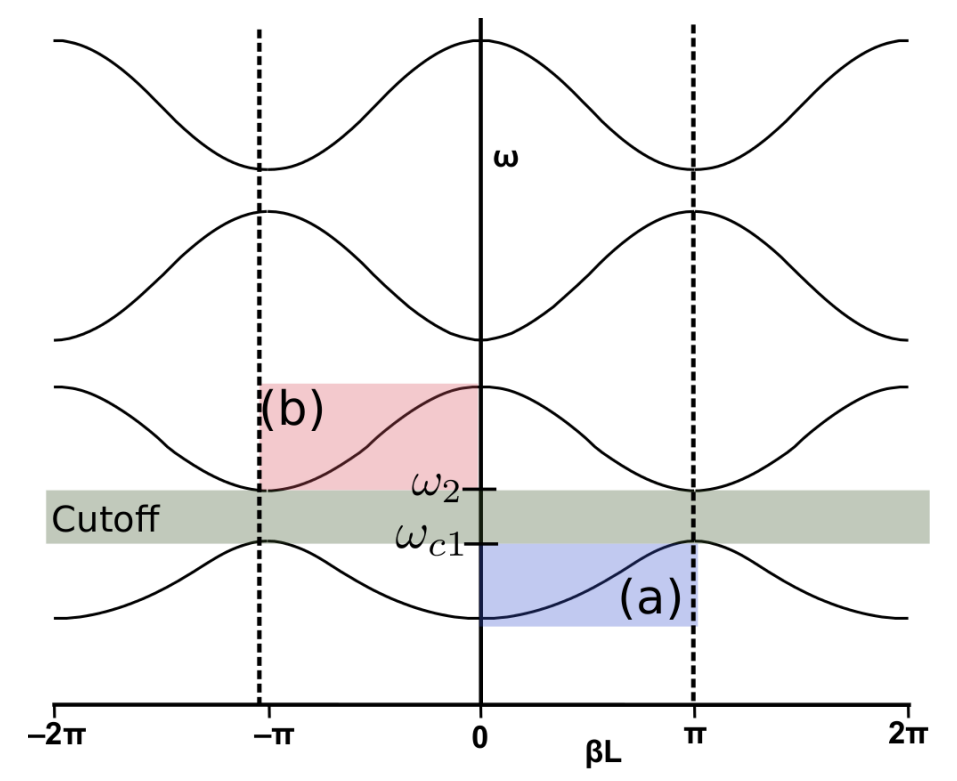

Figure 2.6: Dispersion diagram for periodically loaded rectangular waveguide showing multiple harmonics [2].

It is an important concept to note that power is still transmitted through the device during a backward wave. Power is transmitted in the same direction as the group velocity. The only time where power transmission does not occur is in the cutoff regions. When applying a signal from a source towards a load, the group velocity will always be positive, except during cutoff, and it is the phase velocity which changes direction. This concept will be reiterated during the discussion of the slow wave structure used in this work.

\subsection{Slow Wave Circuits}

Many different slow wave circuits have been developed and studied over the years [7, 44-47]. A very common one used in TWTs is the helical structure. Crossed-Field devices rarely use helical slow wave circuits; however since this type of circuit has 
similar characteristics to the meander line used in this research, a summary is given here.

\subsubsection{Helix}

The helical structure is a conductive coil that resembles a spring. The phase velocity can be estimated by simple geometry. The phase velocity is proportional to the distance traveled in the direction of interest over the total distance the wave traveled. Fig. 2.7(a) shows the helix, and Fig. 2.7(b) shows the helix cut at the points marked as $\mathrm{x}$ and unrolled. Using the view of the helix in Fig. 2.7(b), it is easy to see the phase velocity along the pitch from a wave propagating along the wire at the speed of light, $c$, is given by

$$
v_{p}=c \sin \psi=c \frac{p}{\sqrt{p^{2}+(2 \pi a)^{2}}}
$$

where $p$ is the pitch, $a$ is the helix radius, $c$ is the speed of light, and $\psi$ is the angle of the helix.

The approximation of the phase velocity is rather constant, or non-dispersive, across a wide range of frequencies; hence TWTs which use this circuit have high bandwidth. Deviations from this approximation are due to effective changes in inductance and capacitance when changing the frequency. There are also deviations due to the periodicity of the device when the slow wave wavelength $\lambda_{s w}=p, 2 p$. When $\lambda_{s w}=2 p$, there are two simultaneous modes, a backward wave on the edge of the structure and a forward wave down the center of the helix [2]. This phenomenon is also observed in the meander line circuit studied in this research. 
(a)

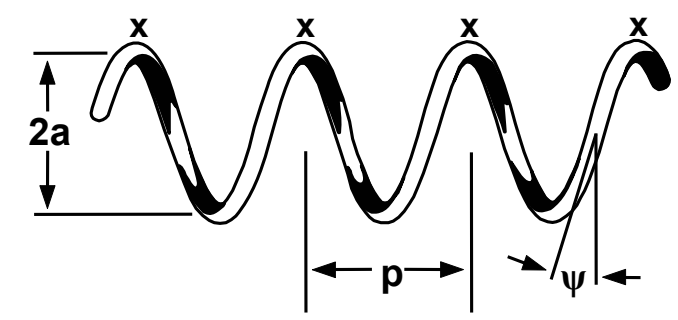

(b)

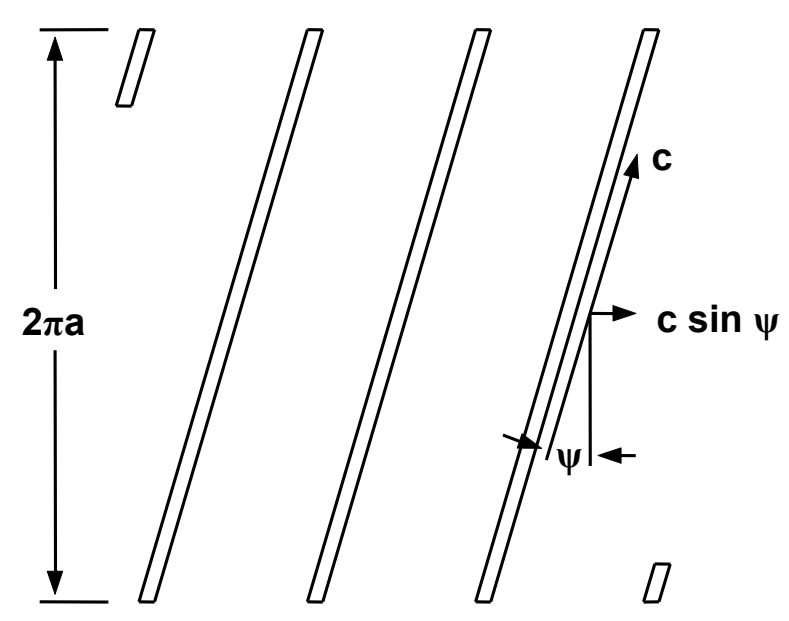

Figure 2.7: Helix (a) and view of helix cut at each $x$ and unrolled (b).[7]

To visualize this backward wave mode, consider Fig. 2.8. Consider an electron traveling left to right and a backward wave traveling right to left along the helical line. In Fig. 2.8(a) the electron feels a force. In Fig. 2.8(b) the electron has traveled to the right $180^{\circ}$, and the backward wave has traveled to the left $180^{\circ}$, and the electron feels no force. In Fig. 2.8(c), the electron has traveled to the right another $180^{\circ}$, and the backward wave has traveled to the left $180^{\circ}$, and the electron feels another force. This mechanism causes causes backward wave oscillations (BWOs) in TWTs. The frequency of backward wave oscillations depend on the beam transit angle from turn to turn, and thus the BWO frequency can be tuned by the beam voltage. These BWOs are caused by electron beams, but the backward wave mode exists when driving high RF frequencies on the helix circuit where $\lambda_{s w}<2 p$. 
(a)

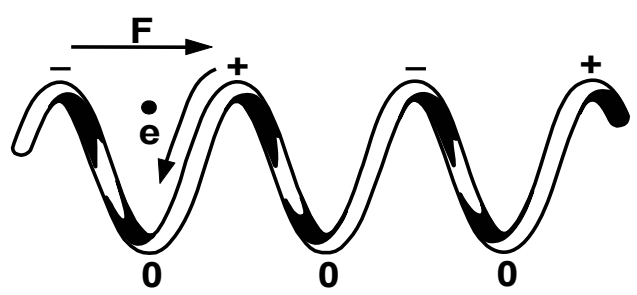

(b)
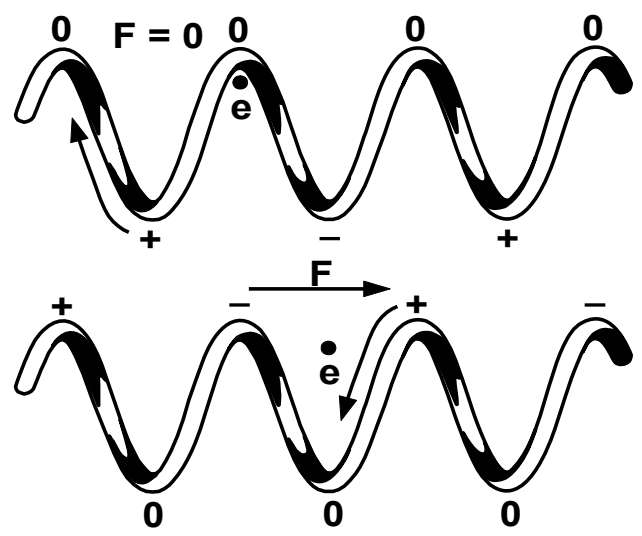

Figure 2.8: Backward wave interaction at two turns per wavelength. [7]

\subsubsection{Meander Line}

The meander line slow wave circuit is used in this research. Figure 2.9 shows an example of a microstrip meander line circuit. The circuit 'zig zags' back and forth in order to retard the phase velocity. Any type of zig zag pattern can be used, but the one shown in Fig. 2.9 is the same as is used in this research. The conductor forms a transmission line over a dielectric and a ground plane. This type of circuit has not been very common for use in crossed field devices, but some interest has increased as of late [48-50]. Because of the flat geometry, one can use well established semiconductor fabrication techniques to manufacture meander lines, which would allow for the creation of tiny slow wave structures $(<1 \mathrm{~mm})$ to allow for higher frequency applications. 


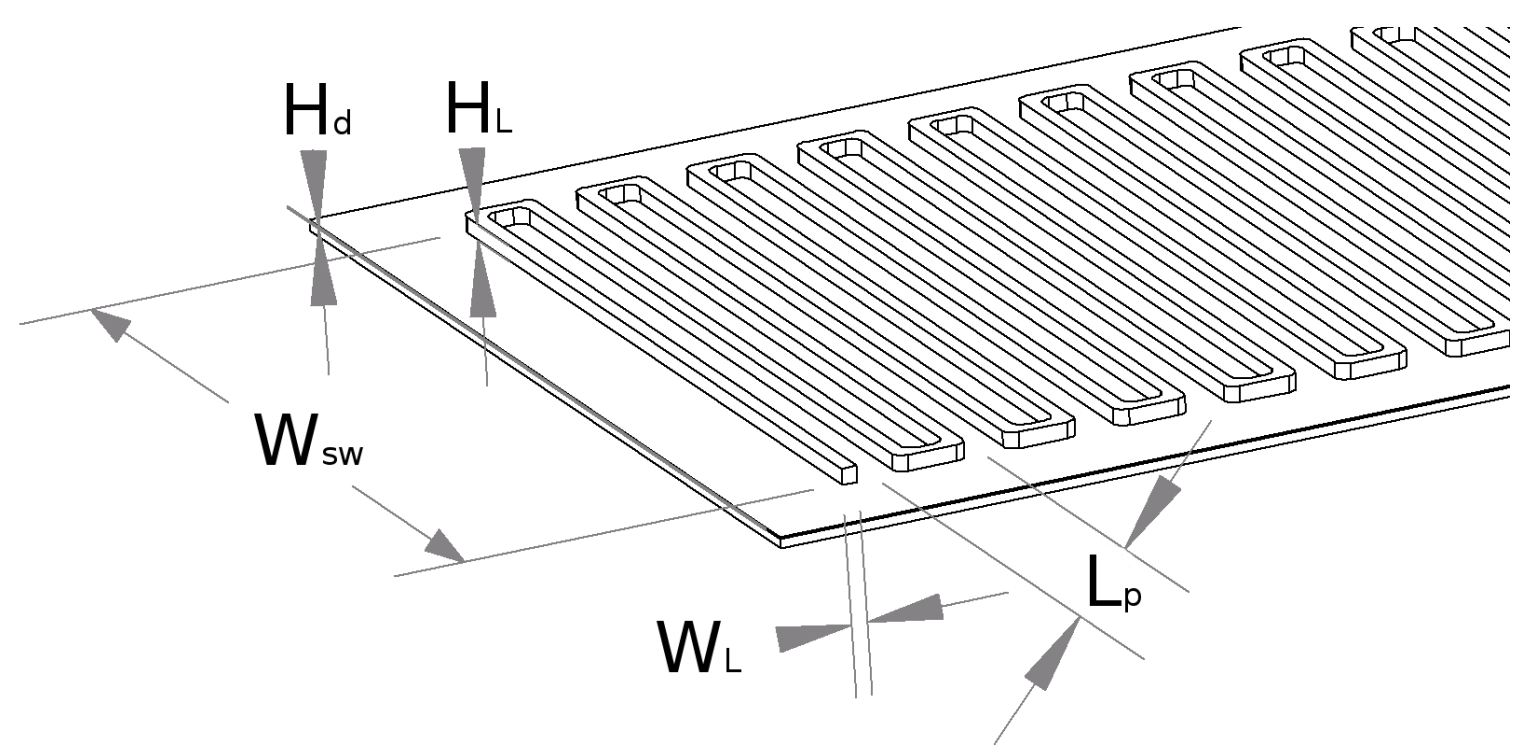

Figure 2.9: A microstrip type meander line, showing a conducting meander circuit over a dielectric material and a ground plane.

Two qualities of the circuit determine the predicted phase velocity: effective dielectric and the geometry. The microstrip has dielectric and a ground plane on one side of the meander line and air on the other. This results in an effective dielectric constant. The equation for the effective dielectric of a microstrip is given in most electromagnetic textbooks and is shown in Eq. (2.2). $H_{d}$ is the dielectric height, $W_{L}$ is the circuit line width, $L_{p}$ is the pitch length, $W_{s w}$ is the width of the slow wave circuit, $H_{L}$ is the meander line height, and $\varepsilon_{r}$ is the permitivity constant for the dielectric. This equation assumes air or vacuum on one side. This effective dielectric determines the velocity retardation, $R_{v}$, of the wave traveling on the microstrip via Eq (2.3). The phase velocity retardation due to the geometry, $R_{\text {geom }}$, is shown in Eq. (2.4). Combining Eq. (2.3) and (2.4) gives the total retardation of the circuit in Eq. $(2.5)$. 


$$
\begin{gathered}
\varepsilon_{\text {eff }}=\frac{\varepsilon_{r}+1}{2}+\frac{\varepsilon_{r}-1}{2 \sqrt{1+12 H_{d} / W_{L}}} \\
R_{v}=\frac{c}{1 / \sqrt{\mu_{0} \varepsilon_{0} \varepsilon_{e f f}}} \\
R_{\text {geom }}=\frac{2 L+L_{p}}{L_{p}} \\
R_{\text {tot }}=R_{v} R_{\text {geom }}
\end{gathered}
$$

There are a few design considerations in the choice of width and length. In order to get good interaction with the electron beam, the length of the circuit must be long; at least six slow wave wavelengths is the arbitrary threshold used here based on prior work [14]. In fact, the longer the circuit, the greater the gain of the device [7], up to a limit.

The second design consideration is operating below cutoff where no backward wave modes exist. This is to prevent mode competition within the circuit. Similar to the helix circuit, a backward wave mode begins at $\beta L_{p}=\pi$, and cutoff is at $\beta L_{p}=2 \pi$. To minimize the complexity of the design, the operation of the meander line will be where $\lambda_{s w}<2 L_{p}$ such that $\beta L_{p}<\pi$. Operation is possible at $L_{p}<\lambda_{s w}<2 L_{p}$; however complicated precautions are needed to prevent amplification of this backward mode, similar to the precautions used in helix TWTs [2]. 


\subsection{Electron Motion}

A CFA contains both static magnetic and electric fields. The force electrons feel from both the magnetic and electric field is described by the Lorentz equation

$$
\mathbf{F}=q(\mathbf{E}+\mathbf{v} \times \mathbf{B})
$$

where $\mathrm{q}$ is the electric charge of the particle, $\mathbf{E}$ is the electric field, $\mathbf{v}$ is the particle velocity, and B is the magnetic field. To visualize this force, Fig. 2.10 shows an electron in a constant E-field, constant B field, and a combined E and B field which are perpendicular to each other. With a constant E field, the electron feels a force in the opposite direction and travels parallel to the field lines, shown in Fig. 2.10(a). With a constant B field, the electron feels a force perpendicular to both the magnetic field and the velocity $\left(U_{0}\right)$ of the electron, and travels in a circle on the plane perpendicular to the B field, shown in Fig. 2.10(b). In a crossed electric and magnetic field, the electron feels forces that are more complex and change throughout the trajectory. The electron travels in a cycloidal trajectory with the average velocity $\left(U_{\text {avg }}\right)$ perpendicular to both fields, shown in Fig. 2.10(c). 


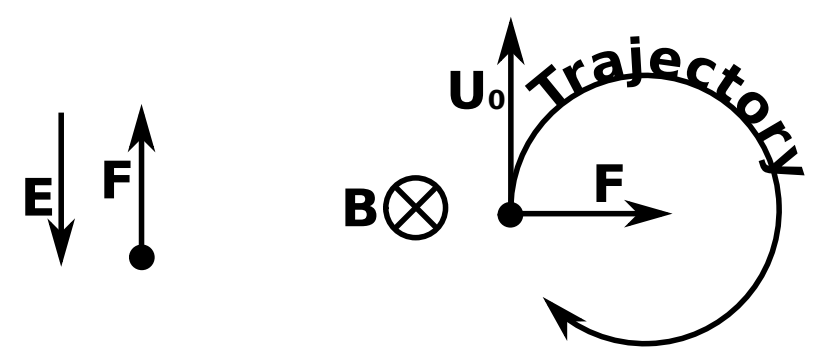

(a)

(b)

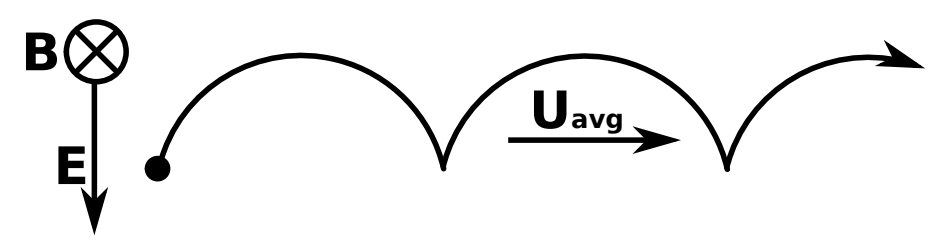

(c)

Figure 2.10: Electron forces and trajectories for (a) a constant electric field and no magnetic field, (b) a constant magnetic field into the page and no electric field, and (c) a constant electric field perpendicular to a constant magnetic field into the page.

The shape of the cycloidal trajectory is altered by the initial kinetic energy of the particle. Fig. 2.11 shows the trajectories of electrons with various initial velocities[2]. 


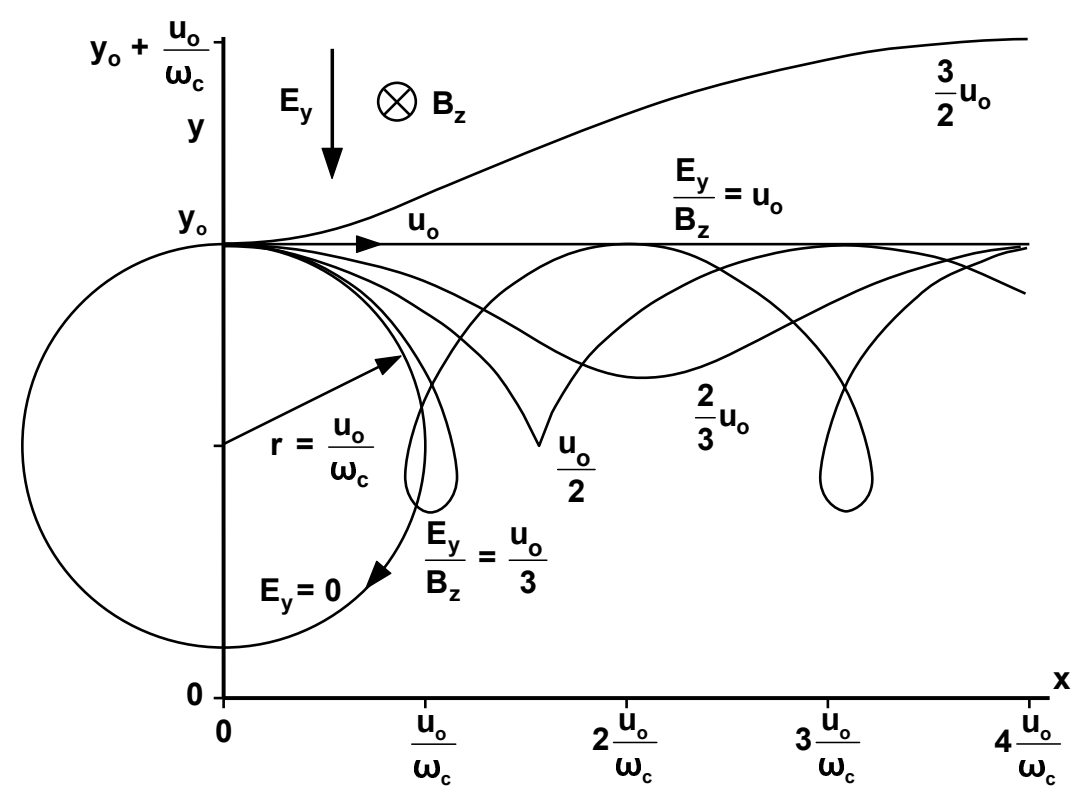

Figure 2.11: Electron trajectories for crossed electric and magnetic fields for various initial velocities $\left(u_{0}\right)[2] . \omega_{c}$ is the cyclotron frequency defined by $\omega_{c}=q B / m$, where $q$ is the particle charge, $B$ is the magnitude of the magnetic field, and $m$ is the particle mass.

A CFA has a static electric field and a perpendicular magnetic field. A cycloidal electron trajectory is observed in CFAs as shown in Fig. 2.10(c). There are 3 parameters of this trajectory that are important: average velocity, the cycloid height, and peak kinetic energy. The average velocity, which is given in Eq. (2.6), or guiding center motion is important because the beam must be able to travel close to the same velocity as the RF wave on the circuit for proper interaction. The height of the trajectory is important because the trajectory must not hit the anode so the electrons can travel down the tube (Hull cutoff condition) [51]. The kinetic energy is important because the velocity of the particle in the direction of wave propagation must be equal to the velocity of the $\mathrm{RF}$ wave at at least one point in the trajectory (Hartree condition) [1]. 


$$
\mathbf{v}=\frac{\mathbf{E} \times \mathbf{B}}{|\mathbf{B}|^{2}}
$$

The Hull cutoff condition describes the threshold voltage or magnetic field for which all the injected current goes to the anode in a crossed field device. For an electron born at the cathode with zero velocity in a linear format, the Hull cutoff voltage $\left(V_{h c}\right)$ and magnetic field $\left(B_{h c}\right)$ are given by Eqs. (2.7) and (2.8) respectively. $d$ is the anode-cathode distance, $B_{0}$ is the magnetic field, $e$ is the electron charge, $m$ is the electron mass, and $V_{0}$ is the voltage between the anode and cathode.

$$
\begin{aligned}
V_{h c} & =\frac{1}{2} \frac{e}{m} B_{0}^{2} d^{2} \\
B_{h c} & =\frac{1}{d} \sqrt{2 \frac{m}{e} V_{0}}
\end{aligned}
$$

The Hartree condition describes the voltage and magnetic threshold at which interaction with the RF wave can occur. For interaction to occur, the velocity on the electron hub surface must be greater than the phase velocity of the wave. If the velocity of the electrons at the hub surface is less than the phase velocity of the RF wave, energy will be transfered to the electron beam and no amplification can occur. The Hartree condition for a linear format device is given in Eq. (2.9), where $\beta$ is the wave number of the RF wave and $\omega$ is the angular frequency.

$$
V_{h}=\frac{\omega B_{0} d}{\beta}-\frac{m}{2 e} \frac{\omega^{2}}{\beta}
$$

The operation of crossed-field devices is between the Hull cutoff and the Hartree line. Figure 2.12 graphically shows the operation region of a magnetron in terms of voltage and magnetic field; this is the same operation region for CFAs. 


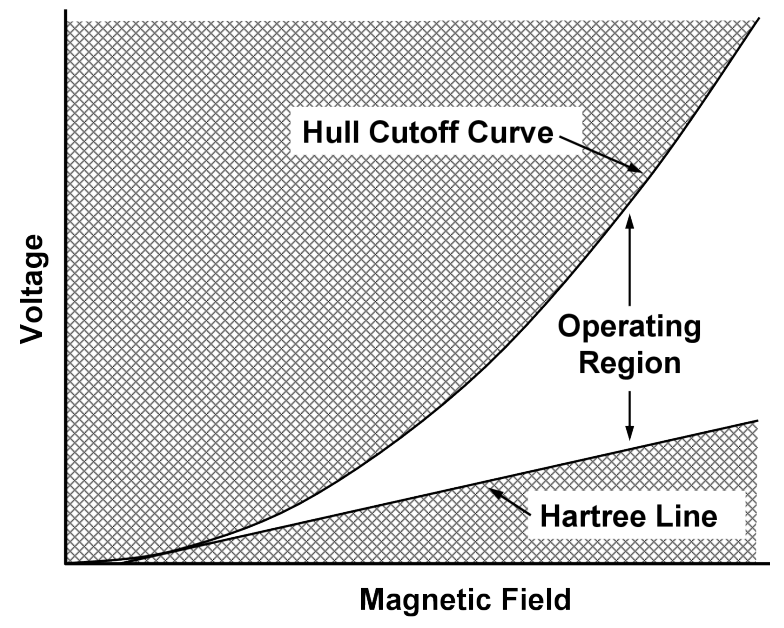

Figure 2.12: Voltage vs. magnetic field showing the operation region of magnetron [2], which is the same for a CFA.

\section{$2.5 \quad$ RF-Beam Interaction}

Figure 2.13 shows the electron beam interaction with an RF wave for a CFA in a moving frame of reference with the electrons [17]. Electrons in the positive regions above the Hull cutoff voltage, drift towards the anode and give up potential energy to the wave. Electrons in the negative regions below the Hartree voltage gain potential energy and drift towards the sole. These electrons remove energy from the RF wave. If more electrons give up energy rather than remove energy, amplification will occur. Also, these electrons form spokes during this process as can be seen in Fig. 2.13 where the two trajectories converge. 


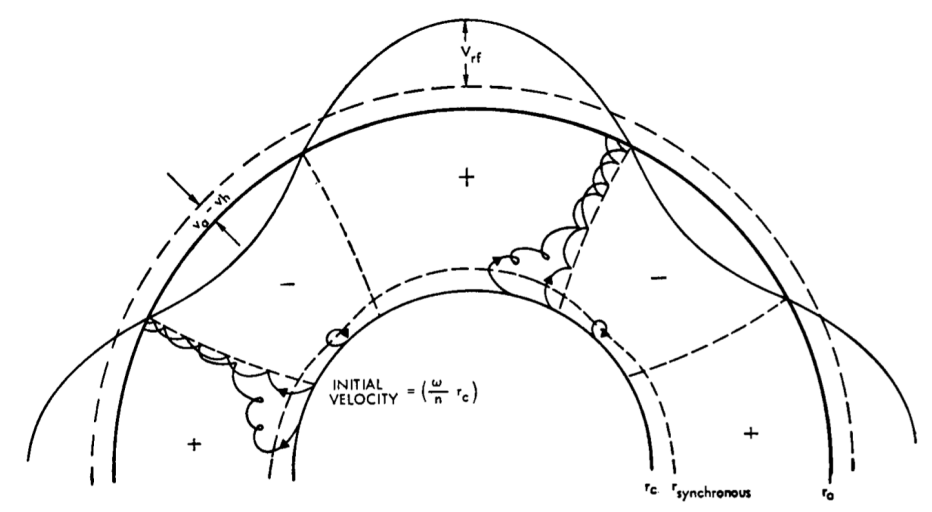

Figure 2.13: Motions of electrons due to the RF field in a rotating coordinate system of a CFA. Electrons in positive RF potentials move towards the sole as they give up energy, and move clockwise into the decelerating region (the region between positive and negative $R F$ potentials where the electric field points clockwise). Electrons in negative RF potentials gain energy, and cycloid right back into the sole. Electrons in the decelerating regions remain in the region but move towards the sole as they give up energy. [17]

The beam and the RF field interaction on the circuit is described by Pierce theory [10]. The reference discusses in detail the interaction in a TWT but also applies similar principles to crossed-field devices. The reference is quite thorough, but for additional insights see $[7,43,52]$. In summary, theoretical gain is predicted by relating two impedances: the interaction impedance, $Z$, and the beam impedance, $Z_{B}$. These impedances relate power to a voltage or electric field and are explained in the following sections.

\subsubsection{Interaction Impedance}

The interaction impedance relates the input power on the circuit to the $x$-component of the electric field created at the beam location in the interaction region. Eq. 2.10 is the formula for calculating the Pierce interaction impedance, $Z_{p}$, where $E_{x}$ is the peak value $x$-component of the electric field at the beam location, $\lambda_{s w}$ is the slow 
wave wavelength, and $P_{i n}$ is the RF input power on the circuit. With a higher $E_{x}$ at the beam location, the higher the impedance, and the greater the interaction. A higher Pierce interaction impedance gives a higher device gain.

$$
Z_{p}=\frac{E_{x}^{2} \lambda_{s w}}{8 \pi^{2} P_{i n}}
$$

The Pierce interaction impedance shown here is the interaction impedance for a TWT, and a different variant is used for crossed-field devices. Eq. 2.11 shows the equation for the interaction impedance for a crossed-field device [52], where $E_{x}$ and $E_{y}$ are the $x$ and $y$ components of the electric field at the beam location, $y_{0}$ is the distance away from the slow wave circuit, $\beta$ is the wave number,and the ${ }^{*}$ denotes the complex conjugate. Far away from the circuit, $Z=Z_{p}$. Note that closer to the circuit, the interaction impedance for crossed-field devices is smaller than the Pierce interaction impedance. The interaction impedances are on the order of tens of $\Omega$.

$$
Z=\frac{j\left(E_{x} E_{y}^{*}-E_{x}^{*} E_{y}\right) \lambda_{s w}^{2}}{16 \pi^{2} P_{i n}}=Z_{p} \operatorname{coth} \beta y_{0}
$$

It can be difficult to analytically determine the interaction impedance of a slow wave circuit, but measuring it is relatively simple. The interaction impedance is found by applying an RF signal to the slow wave circuit terminated into a match load and measuring the $x$ - and $y$-components of the electric field amplitude along the interaction region at the predicted beam location. These measurements can be used in Eq. 2.11 to determine the interaction impedance. With a highly cycloidal beam, the beam location varies along the trajectory. In the highly cycloidal case, the beam location is approximated somewhere within the trajectory. This method to 
measure the interaction impedance can also be used in simulation.

\subsubsection{Beam Impedance}

The beam impedance, $Z_{B}$, is simply the voltage loss of the beam over the beam current. The voltage drop of the beam, often called the beam voltage, is the cathode to anode voltage, $V_{c a}$. Eq. (2.12) gives the beam impedance. Note that as $V_{c a}$ increases, the beam impedance increases, and as $I_{\text {beam }}$ increases, the beam impedance decreases. It is found that a lower impedance leads to higher gain and efficiency and to a more compact device, while high impedance leads to greater stability [53]. Section 2.5.3 describes the effect of beam impedance on the gain and efficiency of the device.

$$
Z_{B}=\frac{V_{c a}}{I_{b e a m}}
$$

\subsubsection{Theoretical Gain}

Both the theoretical gains for the TWT and the CFA are presented here. The gain for a TWT is show in Eq. (2.13) where gain parameter, $C$, is shown in (2.14). The gain for the CFA presented here is from [52] and is shown in Eq. (2.15) where $N$ is the length along the interaction space in slow wave wavelengths and $D$, also called the gain parameter, is shown in eq. (2.16), $\beta$ is the wave number, and $d$ is the anode to sole distance. Both of these equations for gain do not include space charge effects. These equations are only valid in the small-signal-regime of the circuit, before saturation occurs. The gain for the CFA also assumes the beam is non-cycloidal which means the beam is injected at the exact velocity where it moves with a straight trajectory and a constant velocity. 


$$
\begin{gathered}
G=-9.54+47.3 C N \quad[\mathrm{~dB}] \\
C^{3}=\frac{Z_{p}}{4 Z_{B}} \\
G=-6+54.6 D N \quad[\mathrm{~dB}] \\
D^{2}=\frac{|Z|}{Z_{B}}(\beta d)
\end{gathered}
$$

There are a few aspects to note on the gains of the device. The initial gain for the TWT and CFA is $-9.54 \mathrm{~dB}$ and $-6 \mathrm{~dB}$ respectively. Also, the gain increases with the length of the device. The slope of the gain is determined by gain parameters $C$ and $D$ for the TWT and CFA, respectively. Note that the ratio between pierce interaction impedance and beam impedance $\left(Z_{p} / Z_{B}\right.$ and $\left.|Z| / Z_{B}\right)$ are small quantities, less than 1. The gain parameter for the TWT, $C$, is related to this ratio by the $1 / 3$ power, and the gain parameter for the CFA, $D$, is related to this ratio by the $1 / 2$ power. The $1 / 3$ power of a small quantity is larger than the $1 / 2$ power; therefore the gain of the CFA will be less than that of a TWT given similar circuit impedance, current, and voltage.

The gain of the device only depends on the interaction and beam impedances. The gain does not, however, depend on the RF input power of the device. The RF input power will determine where on the circuit the gain saturates. With higher RF input powers, the gain saturates in a shorter length as the energy of the beam depletes. A greater interaction impedance produces a steeper slope. This is because a high 
interaction impedance means there are higher electric fields at the beam location for a specific power. Also, a larger beam current results in a greater gain because more current provides more energy to extract. These two observations are intuitive, but an unexpected observation is that with higher anode to sole voltages $\left(V_{c a}\right)$, the gain decreases. This is unexpected because with higher $V_{c a}$, there is higher beam power and thus more available energy to extract. No physical explanation is given in the references why increasing $V_{c a}$ decreases the gain.

A conceptual attempt at describing the relationship between gain and the beam voltage is provided here. As the beam voltage is increased while keeping the RF wave voltage constant, the relative modulation of the beam caused by the RF wave is diminished. Conceptually, it may be easier to use a TWT example. The beam velocity in a TWT is determined by the beam voltage. With increasing beam voltages, the modulation of the kinetic energy of the electrons relative to the overall beam energy is diminished. With lower relative modulation, the gain decreases. In a CFA, the explanation becomes obscured by the fact that the average kinetic energy of the beam remains unchanged as potential energy is given up to the RF wave and by the fact that the cycloid trajectory constantly shifts between potential and kinetic energy; but the kinetic view of the electron in the cycloid trajectory offers insight into the relative modulation of the electrons during the cycloid trajectory.

There are two interesting observations about the efficiency predicted by Pierce theory. Increasing the beam current not only increases gain but also increases the efficiency of the device. Also, increasing the RF input power increases efficiency. Because the gain is constant for different RF powers, by increasing the RF power, more power can be extracted from the beam for the same length, thus increasing efficiency. The relationship between beam current and efficiency is not immediately 
apparent from the gain equation. Rearranging the terms, Eq. (2.17) shows efficiency. This equation is also difficult to visualize, so Fig. 2.14 shows a plot of the efficiency for the parameters similar to the ones used in the NU CFA for this research where the anode to cathode voltage $V_{c a}=1250 \mathrm{~V}$, the input RF power $P_{\text {in }}=1 \mathrm{~W}$, the interaction impedance $Z=7.4 \Omega$, the anode to cathode distance $d=0.416 \lambda$, and the CFA length in slow wave wavelengths $N=9$. Note that the method to determine the interaction impedance is explained in Sec. 7.4.3. The efficiency increases as the beam current increases. Of course, the limit of this efficiency occurs when the power extracted from the beam gets close to the total power of the beam. Note that the efficiency is very low as is the case for this device from experiment.

$$
e f f=P_{\text {in }} \frac{\left(10^{0.6} 10^{K \sqrt{I_{\text {beam }}}}-1\right)}{V_{\text {ca }} I_{\text {beam }}} \times 100[\%]
$$

where

$$
K=54.6 N \sqrt{\frac{Z \beta d}{2 V_{c a}}}
$$




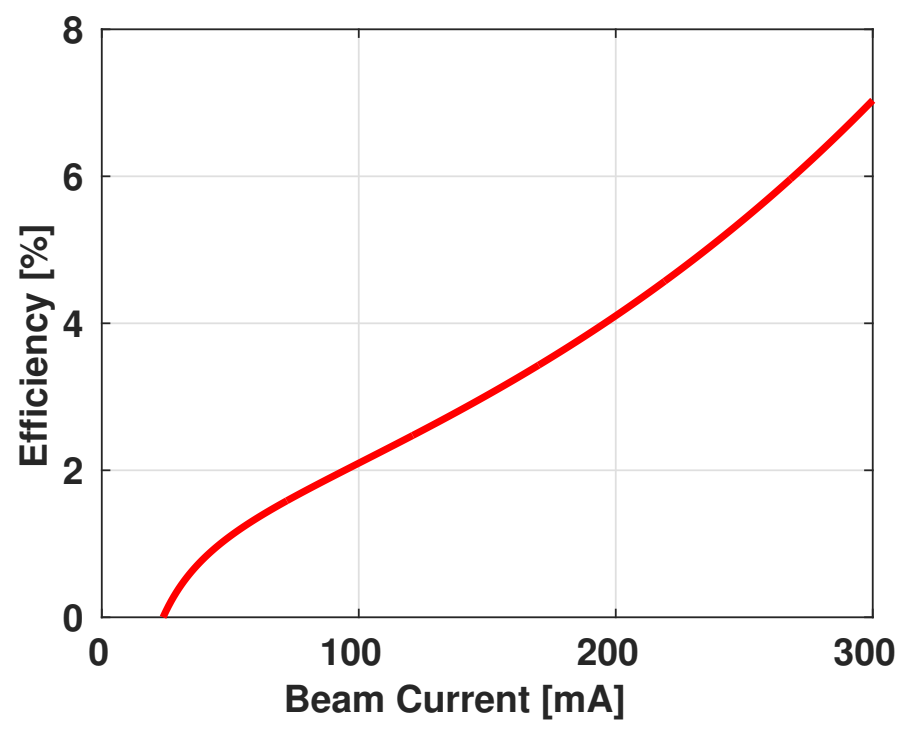

Figure 2.14: Plot of Pierce theory efficiency as a function of beam current for the NU CFA.

\subsection{Electron Sources}

There are two common electron sources used in MVEDs: thermionic and emitting sole cathodes. A less common cathode is a field emitter which is proposed here for reasons described later. A general introduction to each of these sources and a comparison is given here. In order to integrate the GFEAs into the CFA, the use of hop funnels is also proposed, so a brief introduction of hop funnels is given.

\subsubsection{Thermionic Cathodes}

Thermionic cathodes utilize heat in order to emit electrons. Typical cathode temperatures are $\gtrsim 1000^{\circ} \mathrm{C}[1,3]$, and current densities are as high as $\approx 100 \mathrm{~A} / \mathrm{cm}^{2}[1,2]$. By increasing the temperature of the cathode, the number of electrons with sufficient energy to escape increases. The phenomenon is quantum mechanical and requires the use of the energy level diagram for explanation. Fig. 2.15 shows the energy level 
diagram near the surface of a metal. The parabolas represent the energy levels of adjacent atoms. The Fermi level defines the top of the conduction band. The work function is defined as the difference between the energy level of the vacuum and the Fermi level of the metal. In order for electrons to escape the metal, the electron must have an energy greater than the work function of the material.

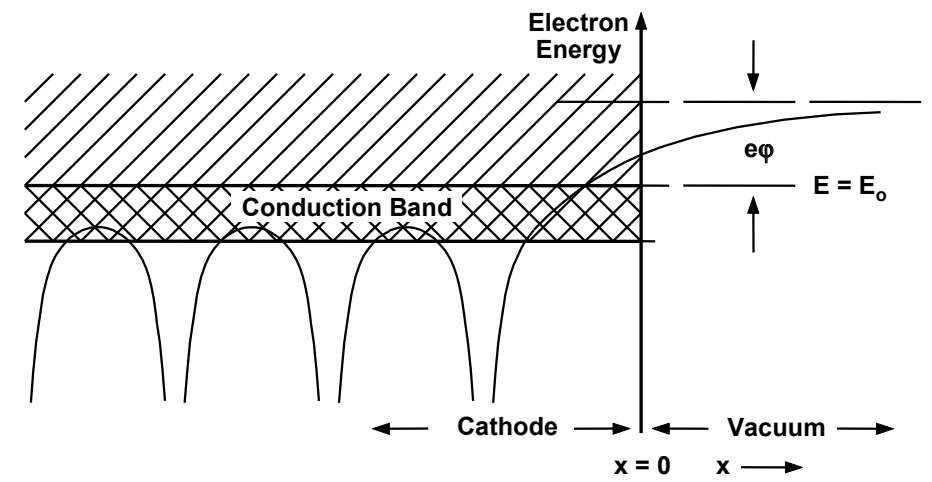

Figure 2.15: Energy Level Diagram near the surface of a metal [2]

The energy of the electrons is defined by the Fermi-Dirac distribution, which is a function of temperature. The average number of fermions in a single-particle state $i$ is given by the Fermi-Dirac distribution, shown in Eq. (2.18) where $\epsilon_{i}$ is the energy of the particle, $k$ is the Boltzmann constant, $T$ is the absolute temperature, and $\mu$ is the total chemical potential. Fig 2.16 shows the Fermi-Dirac distribution for various temperatures. At temperatures of $0^{\circ} \mathrm{K}$, the energy distribution describes that all electrons occupy an energy state below the Fermi level which is where $\epsilon / \mu=1$. At temperatures greater than $0^{\circ} \mathrm{K}, 50 \%$ of electrons occupy a state below the Fermi level and 50\% occupy a state above. Only high energy electrons which are greater than the work function, for example $\epsilon / \mu>3$, of the material, which occupy the tail, are emitted into vacuum. A higher temperature results in a the 'thicker' tail, and it becomes more probable that electrons occupy this very high energy state. 


$$
\bar{n}_{i}=\frac{1}{e^{\left(\epsilon_{i}-\mu\right) / k T}+1}
$$

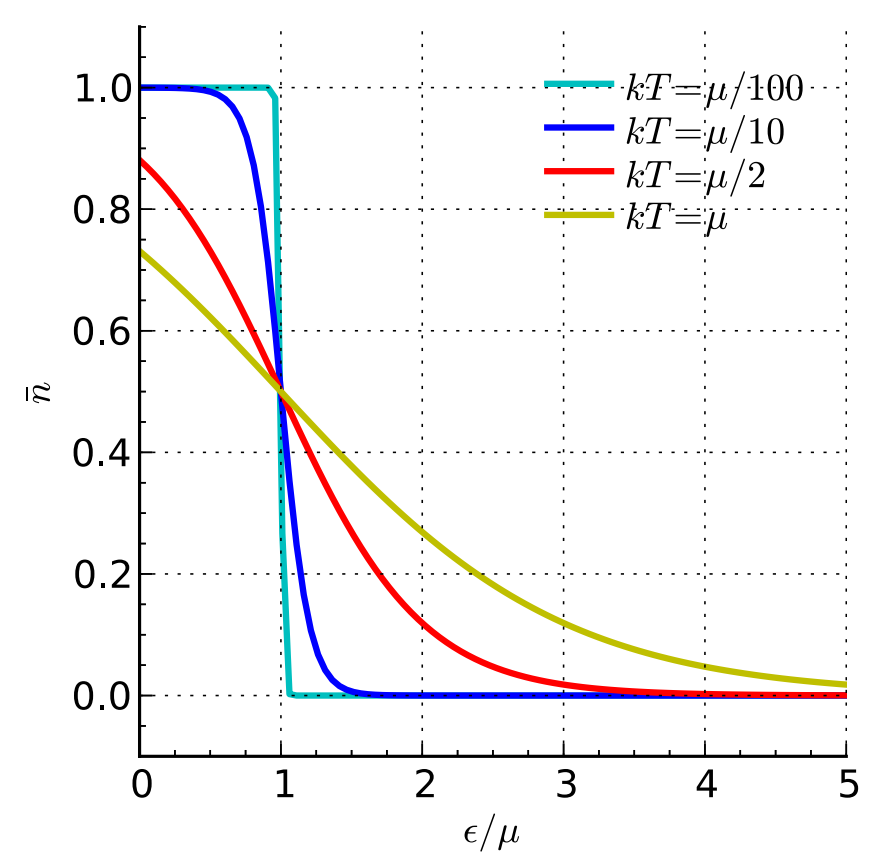

Figure 2.16: Fermi-Dirac distribution for various temperatures.

Using the work function and the Fermi-Dirac distribution function, the current density, $J$, of a thermionic cathode is given in Eq. 2.19. $A_{0}$ is the thermionic emission constant, $T$ is the temperature, $e$ is the electron charge, $\phi$ is the work function of the material, and $k$ is the Boltzmann constant. This equation is known as the Richardson-Dushman equation. As the temperature of the cathode increases, so does the current until the space charge limit is reached. The voltage at which space charge limits the current from the cathode is known as the Child-Langmuir Law and is shown in Eq. (2.20). $P$ is the perveance and defined in Eq. (2.21), where $A$ is the emission area, $d$ is the anode-cathode distance, $e$ is the electron charge, $m$ is 
the electron mass, and $\varepsilon_{0}$ is the permitivity. The space charge limited regime is caused by the negative charge from the emitted electrons depressing the electric field near the cathode, where at a certain charge density the electric field suppresses any additional electron emission. The space charge limited regime the electron emission density is limited by the electric field near the cathode. The Richardson-Dushman equation describes electron emission in the temperature limited regime, the regime where electron emission is limited by the temperature of the cathode. Note that the space charge limit is not only a limit on thermionic cathodes but on all cathodes and is very important in the CFA operation in this dissertation.

$$
\begin{gathered}
J=A_{0} T^{2} e^{-e \phi / k T} \\
I=P V^{3 / 2} \\
P=\frac{4 \sqrt{2}}{9} \varepsilon_{0} \sqrt{\frac{e}{m}} \frac{A}{d}=2.33 \times 10^{-6} \frac{A}{d^{2}}
\end{gathered}
$$

There are two ways to increase thermionic electron emission from a material: increase the temperature of the device or lower the work function of the material. The maximum temperature and the work function are determined by the material, so the choice in material for thermionic cathodes is important. The choice boils down to a balance between the work function and the melting point of materials. Unfortunately, many low work function materials also have low melting points [3]. 


\subsubsection{Emitting Sole}

Emitting sole cathodes utilize secondary electron emission to create the electron beam. This requires a discussion about secondary emission yield (SEY) first and then of the emitting sole cathode.

\subsubsection{Secondary Electron Emission}

Secondary electron emission is a phenomenon in which an electron collides with the surface of a material and one or more 'new' electrons are emitted. There are two primary variables that determine the number of electrons emitted per electron collision: incident energy and angle of incidence of the primary electron. A typical plot of the secondary emission yield $(\mathrm{SEY}), \delta$, versus the primary electron energy is shown in Fig. 2.17.

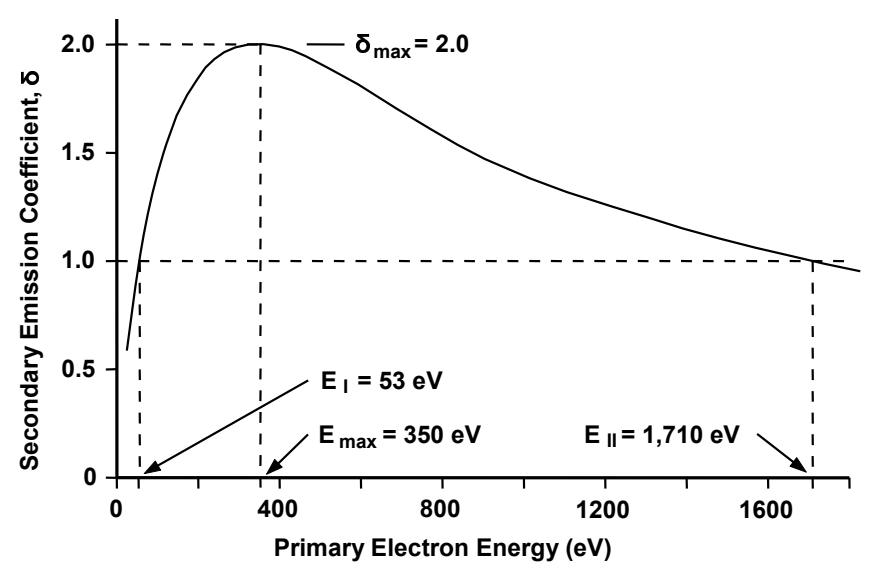

Figure 2.17: A typical secondary electron yield curve for an arbitrary material [2].

There are four primary points of interest on the curve:

1. The lowest primary energy at which the SEY is unity called the first crossover energy, $E_{I}$ 
2. Maximum secondary electron yield, $\delta_{\max }$

3. The primary energy, $E_{\max }$, where the maximum secondary electron yield occurs

4. The highest primary energy at which the SEY is unity called the second crossover energy, $E_{I I}$.

The shape of the curve is universal for all materials, metal or insulator; however the four primary qualities do change from material to material. The two important qualities that are of importance to emitting sole cathodes are $\delta_{\max }$ and $E_{\max }$. Emitting sole cathodes desire a very high SEY in order to get the most current. $E_{\max }$ is important because many emitting sole designs do not use a thermionic cathode and require the $\mathrm{RF}$ field to initiate the current. A low $E_{\max }$ requires a lower $\mathrm{RF}$ power to initiate the current. The first crossover energy is important to the operation of electron hop funnels as discussed later.

The angle of incidence of the primary electron is also important to the SEY. Shallower impact angles yield more secondary electrons. At shallower angles, the primary electron stays closer to the surface and gives up its energy to electrons closer to the surface, and then these electrons have a higher chance to escape the material due to the smaller distance of the escape path.

The energy of the emitted secondary have relatively low energies $(\lesssim 10 \mathrm{eV})[2]$. Any electrons that are emitted near the energy of the primary electron are due to electron backscatter, which is a highly elastic collision. The low energy electrons are referred to here as secondary electrons, and electrons with energies near the primary electron energy are referred to as backscattered electrons. 


\subsubsection{Emitting Sole Operation}

The cold cathode emitting sole operation is represented in Fig. 2.18. By applying energy to an emitting sole device, electrons are slammed into the cathode. From that one electron collision, multiple secondary electrons can be formed, which also can strike the cathode and produce their own secondary electrons. In this way, starting with one electron and some input energy, many more electrons can be generated over a short distance.

\section{$<1 \mathrm{~ns}$}

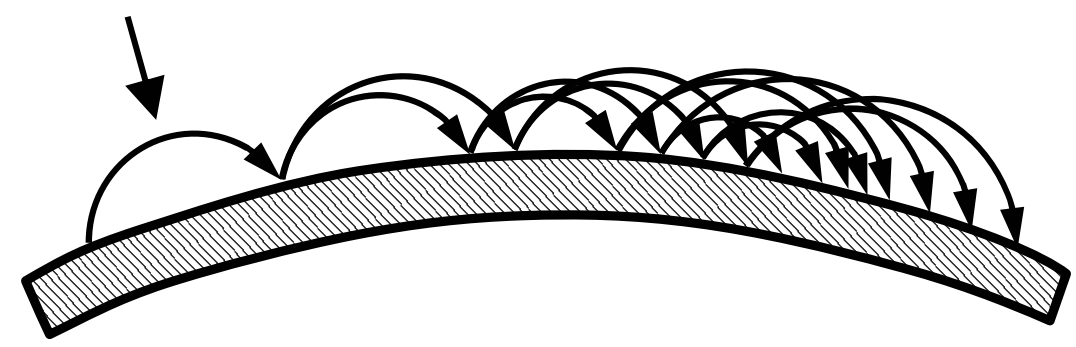

Figure 2.18: Diagram showing the 'multiplication' of electrons on the surface of an emitting sole cathode[2].

Emitting sole cathodes can be used in conjunction with an alternate cathode source or by itself. A thermionic cathode can be used to initiate the beam, and any electrons which gain energy from the RF wave, collide with the sole and produce new electrons which will increase the current density and improve efficiency. Note that electrons which collide with the cathode are in an unfavorable location to give up energy to the RF wave. A few mechanisms exist in which these out of phase electrons shift their phase to a favorable location to give up energy to the RF wave, which is explained in [54]. Emitting sole designs, which do not use an alternate source, initiate the current using the input RF signal to accelerate 'stray' electrons into the cathode. 
The choice in cathode material is important. A material with high $\delta_{\max }$ is desired, and it must be able to withstand the heating due to the electron back bombardment. Also, the surface should be inert in order to maintain consistent operation for the lifetime of the CFA. Slight changes in surface can cause significant changes in the SEY properties of the material, and special precautions must be made in order to maintain the desired SEY. Diamond film on a molybdenum substrate has extremely high $\delta_{\max }\left(\delta_{\max }>20\right)$ but degrades rapidly to a yield of unity due to ion and electron bombardment, which makes their use questionable [2]. More common values of $\delta_{\max }$ of materials used in CFAs are around 2, such as Beryllium oxide $\left(\delta_{\max }=2-2.8\right.$ depending on the surface quality) $[2,55]$, platinum $\left(\delta_{\max }=2.2\right)[56,57]$, and Tungsten Oxide $\left(\delta_{\max }=2.3\right)[2,58]$.

\subsubsection{Field Emitters}

Field emitters utilize strong electric fields along with sharp geometric contours to emit electrons from a conducting material into vacuum via the tunneling effect [7]. There are two general types of field emitters: gated field emitters and field emitters with no gate. Fig. 2.19 shows the configuration of a gated field emitter. A non-gated field emitter is one without the control electrode in the figure. Ungated emitters operate in a diode configuration in which the anode to tip voltage causes field emission. In gated field emitters, the control electrode is used to control the emission current, which makes them more flexible for use as a cathode. At a certain threshold, when the electric field at the emitter tip is on the order of $10^{9}-10^{10} \mathrm{~V} / \mathrm{m}$, the emission current increases rapidly due to the tunneling effect. At very small geometries, only a modest voltage $(\approx 100 \mathrm{~V})$ is needed to create the electric fields necessary for field emission. 


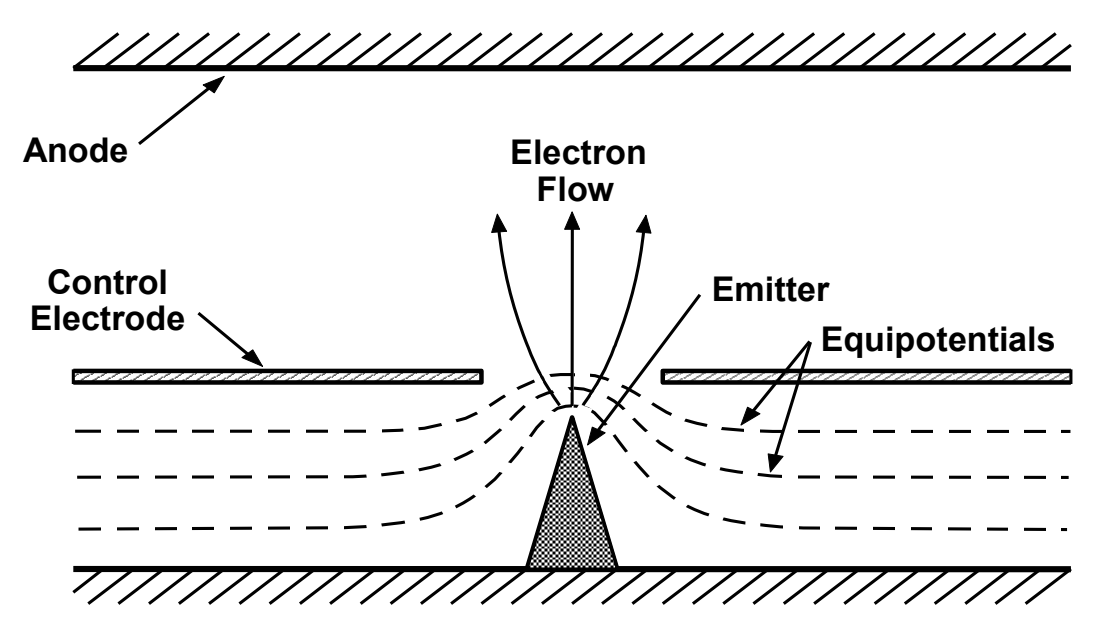

Figure 2.19: Gated field emitter diagram showing the field enhancement near the needle tip [2].

The current density near the emitter tip is in the $10^{6}$ to $10^{12} \mathrm{~A} / \mathrm{cm}^{2}$ range [33]. By having an array of $10^{7}$ tips $/ \mathrm{cm}^{2}$ theoretical current densities of $1000 \mathrm{~A} / \mathrm{cm}^{2}$ are possible. Maintaining this high current density over a broad area, however, is difficult to achieve, and current densities of $20 \mathrm{~A} / \mathrm{cm}^{2}$ at $120 \mathrm{~mA}$ [9] are currently achievable. Recent results have demonstrated current densities of $100 \mathrm{~A} / \mathrm{cm}^{2}$ using silicon tips by Guerrera et. al. [25-27]. These new GFEAs from Guerrera are used as a model of the GFEAs used in this dissertation, so a summary of that work and a discussion on relevant characteristics will be discussed in Sec. (2.6.3.2). But first, a discussion on the physics and the issues of FEAs is presented.

\subsubsection{FEA Physics and Practical Issues}

To understand the relationship between the electric field and electron emission from the surface of the material, the energy level diagram of the material at the surface is used. Figure 2.20 shows the energy level diagram at the surface of a material with and without an applied electric field. 


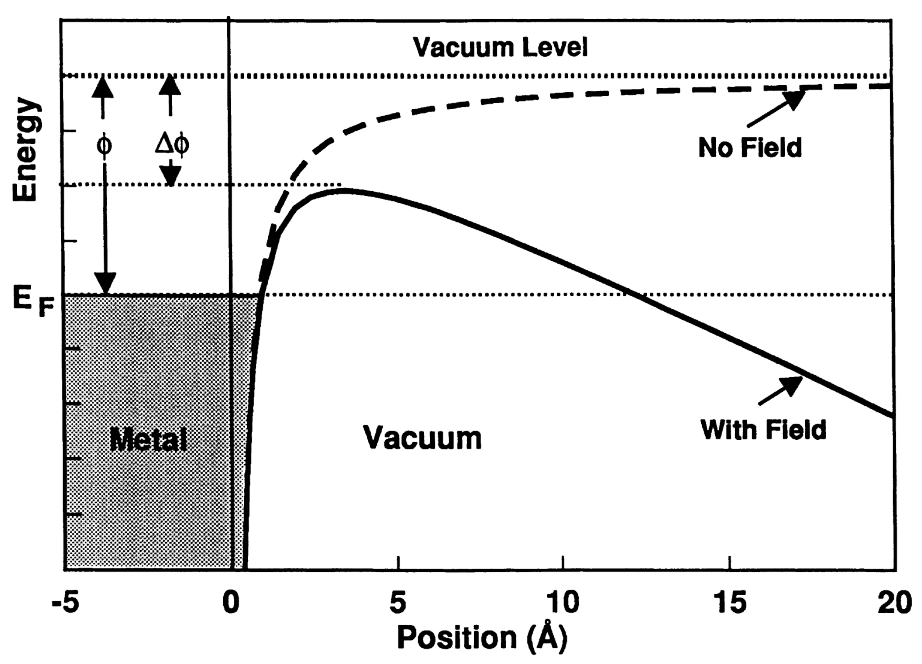

Figure 2.20: Energy level diagram at the surface of a material with and without an applied electric field [24].

The dashed line is the energy level diagram for the material with no electric field. Without the electric field, an electron must overcome the potential barrier given by the work function of the material, $\phi$. By applying an electric field, a lowering of the potential barrier is observed, $\Delta \phi$. With high electric fields, the energy barrier becomes very narrow. Because of the wave like nature of the electron, there is a probability that the electron exists in the vacuum side of the barrier even though it does not have sufficient kinetic energy to escape, which is called the tunneling effect. At a certain threshold, when the electric field is on the order of $10^{9}-10^{10} \mathrm{~V} / \mathrm{m}$, the current increases rapidly due to this effect. The current density $(J)$ is found by using the shape of the energy barrier, integrating the probability function of an electron tunneling through the barrier, and multiplying by the electron supply function. The current density due to an applied electric field is shown in Eq. (2.22). This equation is known as the Fowler-Nordhiem equation. 


$$
J=1.42 \times 10^{-6} \frac{E^{2}}{\phi} \exp \left(\frac{10.4}{\phi^{1 / 2}}\right) \exp \left(\frac{-6.44 \times 10^{7} \phi^{3 / 2}}{E}\right)
$$

There are many benefits of the gated field emitter over thermionic and emitting sole cathodes. The low transconductance, low capacitance, and small gate-emitter gap allow for a fast modulation of the electron beam. Modulation frequencies of $10 \mathrm{GHz}$ are currently achievable [32]. Also, because there is no need for a heating assembly or very high voltage supplies, the efficiency of GFEAs are higher, and smaller assemblies are possible. Another advantage is that the construction of a cathode with highly resolved spatial control and integration in an MVED is much easier than using a thermionic cathode. For these reasons, GFEAs are proposed in this work.

The main issue with GFEAs are their susceptibility to ion back bombardment and oxidation. Even at very low pressures for conventional tubes $\left(10^{-7}-10^{-9}\right.$ Torr $)$, desorbed gas neutrals from electron bombardment/heating are ionized by the emitted electrons which then accelerate back towards the cathode and can damage the emitter tip. This lowers the field enhancement factor which degrades the performance. Oxidation also can occur on the emitter tips. This phenomenon increases the work function of the emitter which also degrades the performance. Oxidation, however, is reversible to a certain extent [24].

Beam uniformity and beam convergence is another concern with FEAs. Creating an array of consistent emitter tips is difficult. Because of this, the current densities vary from tip to tip and cause a non-uniform current density. Also, due to the emission characteristics and the lack of focusing at the emitter tips, a high transverse velocity component in the electron beam is expected. High current densities at the emitter tip can cause some space charge defocussing as well.

With the current GFEAs, integration with MVEDs is difficult, mainly due to the 
low lifetime of the emitters at high current densities. Even so, lower power devices have been described [59]. Also, considerable improvements have been made in GFEA research in the past 20 years, and GFEA improvements are expected.

\subsubsection{Discussion of Silicon GFEAs Fabricated at the Massachusetts Institute of Technology}

Field emitters developed at MIT [25-27] have shown many desirable characteristics for use in MVEDs and are a promising candidate for current modulation. Current densities $>100 \mathrm{~A} / \mathrm{cm}^{2}$ with gate-to-emitter voltages $<75 \mathrm{~V}$ have been demonstrated with lifetimes $>100 \mathrm{~h}$. The low gate-to-emitter voltage is of special note because it provides an easy way to modulate the current with relatively low power. The I-V curves for various array sizes are shown in Fig. 2.21. The current varies exponentially with voltage. With a change of roughly $15 \mathrm{~V}$, the current varies by about 2 orders of magnitude. 


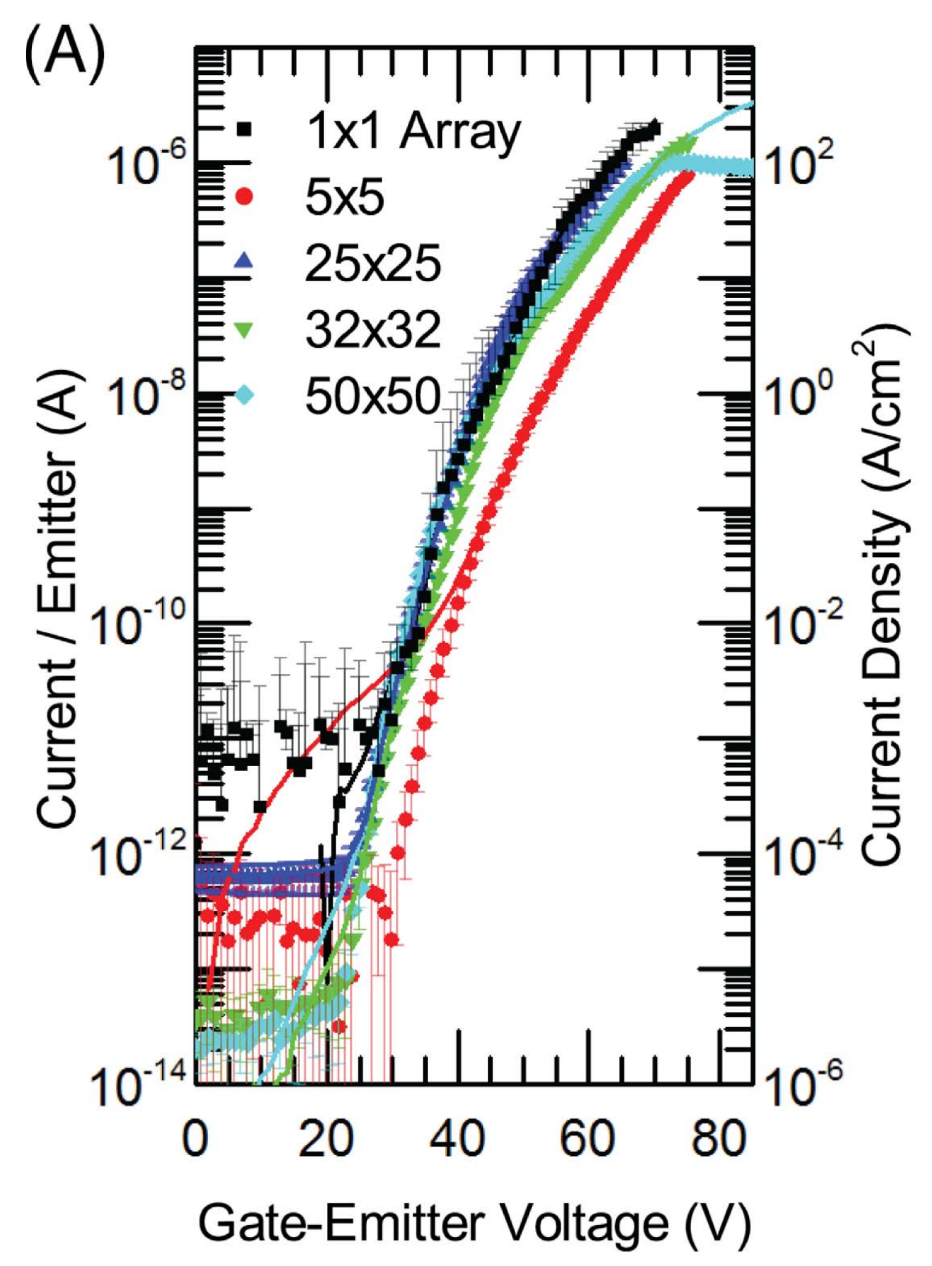

Figure 2.21: The current density vs. the gate emitter voltage of the gated field emitters fabricated by Guerra et. al. [25]

\subsubsection{Hop Funnels}

Hop funnels are structures made from insulating material which use secondary electron emission to transmit current[36-38]. Fig. 2.22 shows a diagram of an electron hop funnel and the simulated electron trajectories for two different hop electrode voltages. Fig. 2.22a(a) shows a case where the hop funnel transmits current with a hop electrode voltage of $750 \mathrm{~V}$. Electrons emitted from an electron source move up due to the electric field created by the hop electrode and collide with the funnel wall. 
Secondary electrons created from this collision travel up the wall due to the electric field, collide with the wall, and create secondary electrons of their own. This cycle repeats until no secondary electron is created or until the electron leaves the funnel exit. Fig. 2.22b(b) shows an unfavorable condition where transmission does not occur where the hop electrode is $0 \mathrm{~V}$. The electric field created by the hop electrode does not accelerate the emitted electrons above the energy at which the first crossover occurs on the Secondary Electron Yield (SEY) curve. These electrons produce less than 1 secondary electron per collision and eventually charge the funnel wall negatively. This negative charge accumulates and counteracts the electric field of the hop funnel and repels the electrons back towards the cathode.

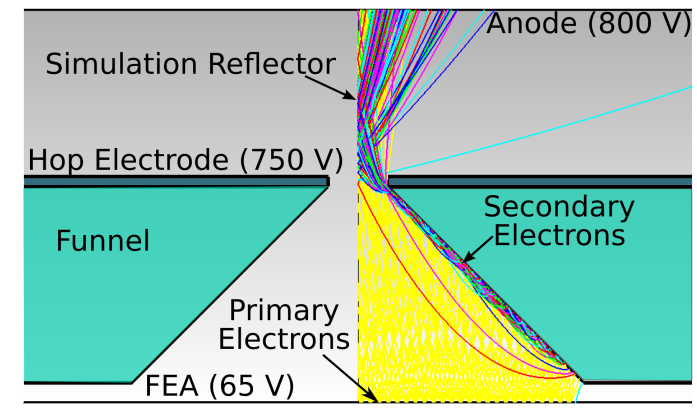

(a)

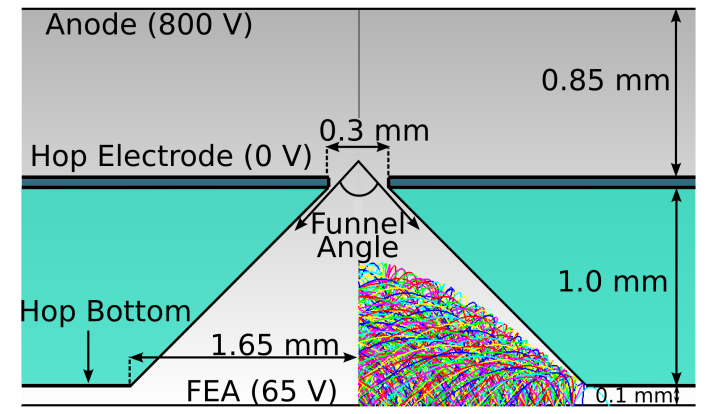

(b)

Figure 2.22: Hop funnels used in [37], showing the operation during (a) full electron transmission and (b) no transmission using the Lorentz $2 \mathrm{E}$ [60] simulation.

The majority of the electrons leaving the funnel are born at the potential of the hop funnel wall [37]. The average kinetic energy of the electrons when they are born is approximately $5 \mathrm{eV}$, determined by secondary electron emission. Using this property of hop funnels, one can control the energy of the electrons leaving the source. Also, by adding another electrode, one can control the potential outside the funnel, while 
having separate control of the energy at which the electrons are born. Figure 2.23 shows this design. The hop electrode controls the energy at which the electrons are born, and the sole electrode controls the potential which is seen from anywhere above the funnel structure. This is the design proposed for the distributed cathode in the CFA. By biasing the hop electrode less negative than the sole, electrons cycloidal in the interaction region are born at a potential less negative than the sole. Without the RF wave, this prevents the cycloidal electrons from being collected on the sole.

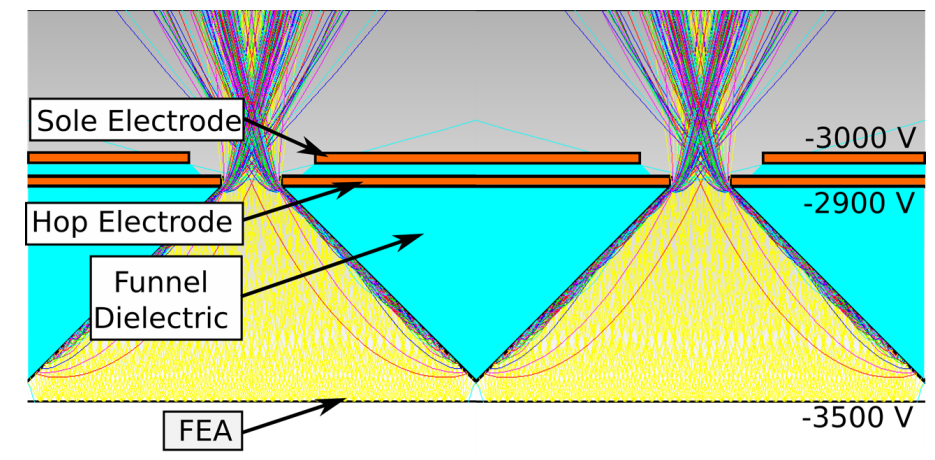

Figure 2.23: Hop funnel structure with sole electrode using Lorentz 2E [60]

\subsection{Simulation}

Three simulations are used in this work: COMSOL [41], SIMION [40] and Vsim [42]. COMSOL is a finite element solver; SIMION is an electrostatic particle trajectory code; and Vsim is a finite difference particle in cell (PIC) code. This software will be discussed in more detail in this section

\subsubsection{COMSOL}

COMSOL is a very user friendly multi-physics solver which uses the finite element method. One can build complex geometries, create complex mesh, and solve many 
different problems all in one program. To build a model, one must create the geometry, mesh the geometry, and then choose the appropriate solvers.

COMSOL has many different features to create the geometry. These are very similar to many cad type programs, and the features are not discussed in detail here. General features include: workplanes, rectangles, spheres, etc.

The meshing algorithm allows individual control of regions or boundaries and of the mesh technique. There are two types of mesh: structured and unstructured. The unstructured mesh used here consists of tetrahedrons in the region with triangles on the boundaries. The mesh can also be non-uniform, meaning that the mesh can change size in order to resolve very small features without the cost of resolving regions which need fewer elements.

Three important meshing parameters are the minimum mesh size, the maximum mesh size, and the growth rate. The minimum mesh size prevents the generation of too many elements which limits the memory usage. The maximum mesh size limits the error of the model and ensures that the features of the geometry of the $\mathrm{RF}$ wave can be resolved. The growth rate parameter limits the change in size of the adjacent elements. Mesh quality can be controlled from these mesh parameters. Having too large of a mesh and having large growth rates introduces error. The mesh should be the smallest size and the smallest growth rate within computational memory constraints.

COMSOL has many different solvers that can be used to study a problem: timedependent, stationary, or eigenfrequency solver. The time dependent solver is used to see phenomena development in time. The stationary solver determines the steady state of the phenomena. The eigenvalue solver determines the natural harmonic oscillations of a time dependent problem. COMSOL was used to determine the 
dispersion and the standing wave pattern of the meander line slow wave circuits used in the CFA designs. Because only the steady state solution is needed to determine the standing wave pattern, the stationary solver is used.

There are many different algorithms that can be used. The algorithms are broken up into two categories: direct solvers and iterative solvers. Direct solvers directly solve the system of linear equations using a LU factorization (lower upper factorization) method. These solvers generally use a lot of memory and can be slow. Iterative solvers start with an initial 'guess' of the solution and iteratively make new 'smart guesses' that are hopefully closer to the actual solution. When the error between the iterations becomes smaller than a convergence criterion, the solution is considered to be found. Iterative methods use less memory and can be faster. There are many different iterative solvers, and the difference among them is in the method they use to 'guess' the next iteration.

The study performed in this work is a frequency sweep which uses a stationary iterative solver. The algorithm used in this work is the default, which is a biconjugate gradient stabilized iterative (BiCGStab) method [61]. This method uses the multifrontal massively parallel sparse (MUMPS) direct solver [62] to create the initial guess.

\subsubsection{SIMION}

SIMION is a 3D finite difference particle trajectory code for electrostatic fields. The program has a graphical interface in which one can create the geometry and boundary conditions, create particle sources, and post process all in one program. The code also has a very extensive scripting language to build the geometry, inject particles, 
perform real-time data processing, and perform post processing. This code is used to study the electron optics in the CFA.

The simulation only has a static electric field solver, a static magnetic field solver, and a particle trajectory solver. No electromagnetic waves can be modeled. The geometry or the potentials of electrodes can be altered during simulation, but the code still uses the electrostatic solver. Space charge can also be modeled but is not used in this work.

\subsubsection{Electrostatic Solver}

To create the static electric fields, SIMION uses potential arrays. The user defines the boundary conditions of each electrode or electrodes and assigns them a number. Each unique number corresponds to its own potential array. Each potential array is solved individually to find the electric field at each point. Because of the additive solution property of the Laplace equation, each separate potential array can be added together to find the electric field in the total geometry.

The algorithm to solve for the electric field for each potential array is a dynamically self-adjusting over-relaxation method [63, 64]. This refining algorithm is only called once, unless the user requests otherwise, and the code uses a fast adjust method to alter the electrostatic field upon a change in electrode voltage. After the first solution of a potential array, each point can use a scaling factor to account for a different electrode voltage. After the potential array is updated, it can then be added to the other potential arrays to get the total solution. 


\subsubsection{Magnetic Field Solver}

The code also has potential arrays for static magnetic fields. Magnetic fields are normally represented and measured as gradients, and to utilize a similar solver as the electrostatic, SIMION needs magnetic potentials. The code uses vector magnetic potentials to create magnetic fields. Magnetic 'potentials' can be defined in the potential array and can solve for the magnetic field in the same way as for the electrostatic solver. This is not the best way to solve for the magnetic field because magnetic poles do not have uniform magnetic potentials. Magnetic fields generated by this solver must be carefully studied for accuracy. An external magnetic field can also be input manually. Since the static magnetic field in the CFA is uniform, one can supply a constant for the magnetic field at all points in the particle trajectory.

\subsubsection{Particle Push}

The force on the particles is calculated from the electrostatic field, the magnetic field, and the charge repulsion. Each of the forces are then added together to find the total force. With these forces, the particle trajectories are determined using an adaptive time-step 4th order Runge-Kutta method. It should be noted that the trajectory algorithm is 'blind' to boundaries and sharp gradient edges.

To detect boundaries, on each trajectory calculation, the algorithm detects if an edge is crossed. If the edge is detected, the algorithm tries new time steps until it can approach the wall in an accurate manner. Detecting sharp gradient edges is achieved by testing the coefficient of variation squared for the four Runge-Kutta acceleration terms against an accuracy level [64]. The time step is reduced until all values are less than the upper limit or the minimum time step size has been reached. 


\subsubsection{Vsim}

The term 'particle-in-cell' (PIC) refers to a technique of tracking macroparticles in a Lagrangian frame, while the moments of the distribution such as densities and currents are computed simultaneously on stationary Eulerian mesh points. Macroparticles represent the mass and charge of a large number of single particles. This approximation is used to reduce the computational load by simulating less particles. Vsim is a $3 \mathrm{D}$ PIC code which uses the finite difference method to determine electric fields and magnetic fields and uses these fields to push the particles.

Time-varying electromagnetic fields and electrostatic fields can both be calculated on the finite difference mesh. Any initial space charge in the system due to the charged macroparticles are accounted for in the electrostatic solver. The electric field created by the movements of charged macroparticles are accounted for in the electromagnetic solver. Currents can also be defined in this code, and the electromagnetic fields they create are accounted for in the electromagnetic solver. Charge densities can also be defined and are accounted for in the electrostatic solver.

Many different particle dynamics are also included in the code. Particle to particle interactions, secondary electron emission, and photon emission can all be modeled. Monte-Carlo methods are used to model these phenomena [65-67]. These particle interactions are not used in this work.

\subsubsection{Static electric field solver}

The static electric field solver uses the finite difference grid. Given all the boundary conditions and charge distribution $(\rho)$ the potential $(U)$ within the domain can be solved with Poisson's equation. In vector form, Poisson's equation is given by Eq. 
(2.23). The numerical expression is given by Eq. (2.24), where $u_{i, j, k}$ is the potential at a specific grid point location $U(i \Delta x, j \Delta y, k \Delta z)$, and $\Delta x, \Delta y$ and $\Delta z$ are the spacing between the grid locations in the $x-, y-$ and $z$-directions. Poisson's equation requires an iterative solver, and there are many algorithms which can be used. The choices are bi-conjugate gradients stabilized (bicgstab) [68], conjugate gradient squared (cgs), generalized minimal residual (gmres) [69], conjugate gradients (cg), or transposefree quasi minimal residual solver (tfqmr). The user has control over many other parameters of these algorithms not shown here. To determine the electric fields, the gradient of $U$ is found.

$\nabla^{2} U=\rho$
$\frac{u_{i-1, j, k}-2 u_{i, j, k}+u_{i+1, j, k}}{\Delta x^{2}}+\frac{u_{i, j-1, k}-2 u_{i, j, k}+u_{i, j+1, k}}{\Delta y^{2}}+\frac{u_{i, j, k-1}-2 u_{i, j, k}+u_{i, j, k+1}}{\Delta z^{2}}=\rho_{i, j, k}$

\subsubsection{Electromagnetic Field Solver}

The electromagnetic solver uses the Yee finite difference time domain (FDTD) scheme[70]. The method solves Faraday's and Ampere's laws, shown in Eqs. (2.25) and (2.26), where $\mathbf{E}$ and $\mathbf{B}$ are the electric and magnetic field vectors and $\mathbf{J}$ is the current density.

$$
\begin{aligned}
& \frac{\partial \mathbf{B}}{\partial t}+\nabla \times \mathbf{E}=0 \\
& \frac{\partial \mathbf{D}}{\partial t}-\nabla \times \mathbf{H}=\mathbf{J}
\end{aligned}
$$


In Cartesian coordinates, Eqs. (2.25) and (2.26) are expanded to the following system of scaler equations:

$$
\begin{gathered}
-\frac{\partial B_{x}}{\partial t}=\frac{\partial E_{z}}{\partial y}-\frac{\partial E_{y}}{\partial z} \\
-\frac{\partial B_{y}}{\partial t}=\frac{\partial E_{x}}{\partial z}-\frac{\partial E_{z}}{\partial y} \\
\frac{\partial B_{z}}{\partial t}=\frac{\partial E_{x}}{\partial y}-\frac{\partial E_{y}}{\partial x} \\
\frac{\partial D_{x}}{\partial t}=\frac{\partial H_{z}}{\partial y}-\frac{\partial H_{y}}{\partial z}-J_{x} \\
\frac{\partial D_{y}}{\partial t}=\frac{\partial H_{x}}{\partial z}-\frac{\partial H_{z}}{\partial y}-J_{y} \\
\frac{\partial D_{z}}{\partial t}=\frac{\partial H_{x}}{\partial y}-\frac{\partial H_{y}}{\partial x}-J_{z}
\end{gathered}
$$

The finite difference grid defines electric fields on the middle of the edges, and the magnetic fields in the center of the face. Figure 2.24 shows a grid cell and the various positions of the electric and magnetic field components. These positions are chosen so that the boundary condition for a perfect electric conductor (PEC) on the edge of the cube contains and sets the perpendicular components of the electric field and normal component of the magnetic field to zero. For example, plane surfaces normal to the $x$-axis contain the points where $E_{y}, E_{z}$, and $H_{x}$ are defined. 


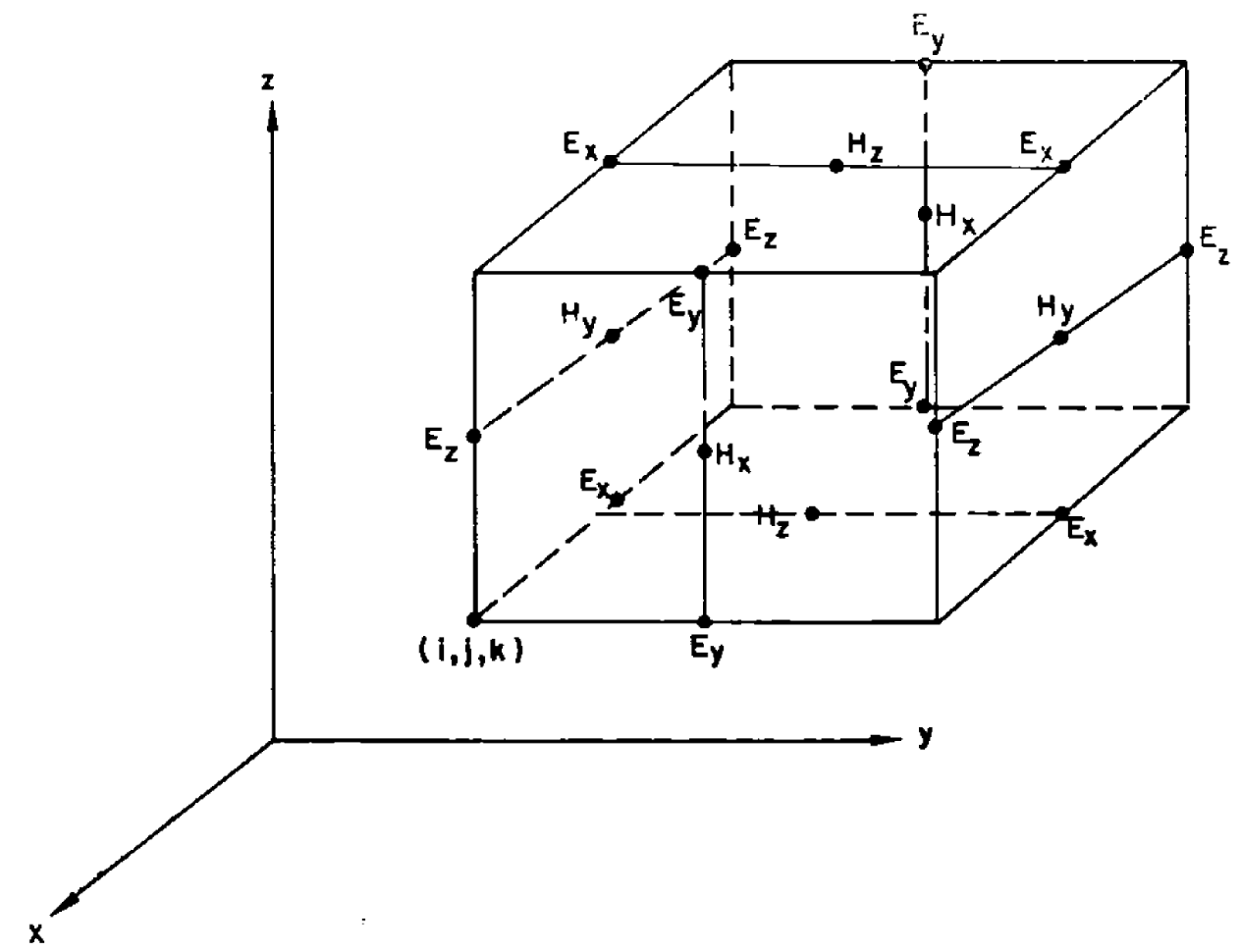

Figure 2.24: Yee grid showing the position of the various field components. Electric field components are on the middle of the edges and magnetic field components are on the center of the faces.

The numerical calculation flow is shown in Fig. 2.25 [71]. First the electric and magnetic fields are initialized to zero. Using Faraday's law, the electric fields are updated in the interior of the domain. Then the boundary conditions are updated. These boundary conditions can be static or time-varying depending on the simulation problem. After the electric fields are updated, the magnetic field is updated using Ampere's Law. This leapfrog approach using the Faraday-Ampere-Faraday-Ampere updating scheme repeats until the maximum timesteps are achieved. 


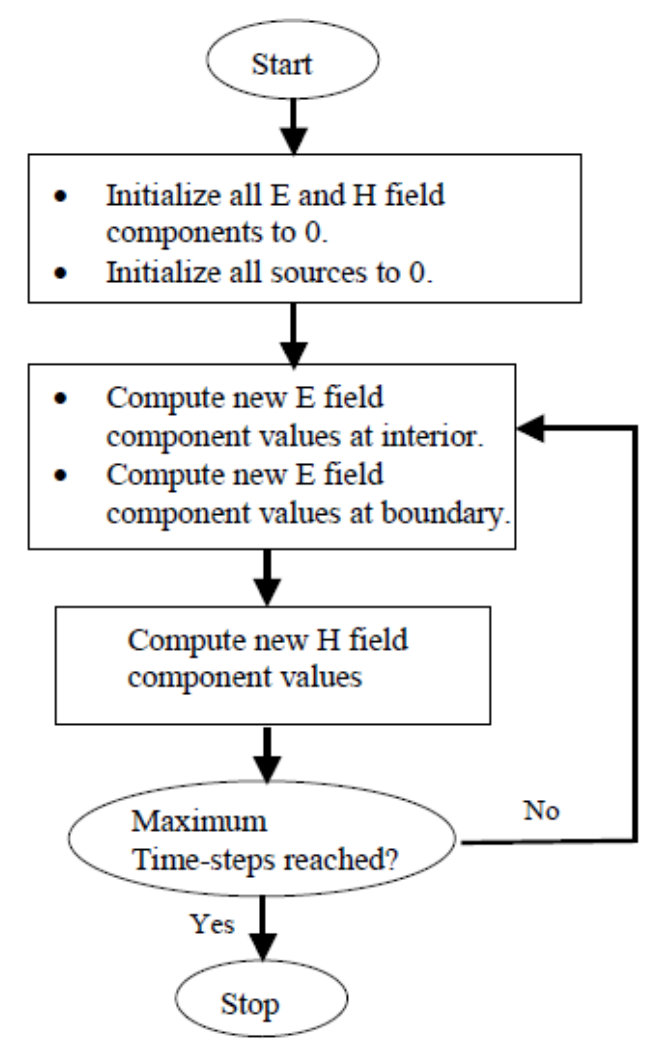

Figure 2.25: FDTD simulation flow [71].

The maximum timestep that can be used with the FDTD scheme is limited by the time it takes light to transmit through a cell known as the Courant-Friedrichs-Lewy (CFL) stability criterion [72]. The Courant condition is shown in Eq. (2.27). With timesteps that exceed the Courant condition, the FDTD scheme is unstable.

$$
\Delta t<\frac{1}{c \sqrt{\frac{1}{\Delta x^{2}}+\frac{1}{\Delta y^{2}}+\frac{1}{\Delta z^{2}}}}
$$

The FDTD grid is a Cartesian or cylindrical grid which approximates every boundary with this grid. Curved boundaries, ones which do not align well with the cell edges, are usually approximated by a stair-step method [73]. This method 
only has a first-order accuracy with grid size. Another method which is available in VSim is the Dey-Mittra [74, 75] cut-cell method. This method has a second order accuracy with grid size. because there are only a few unimportant curved boundaries used in the CFA in this research, the Dey-Mittra method is not used, so a review is not provided.

\subsubsection{Particle Push Algorithm}

To model each electron in an beam would be very impractical due to computational and time constraints. Particles in Vsim are modeled as macroparticles, where one macroparticle has the charge and mass of many particles. To move the particles, the code uses the Boris-Push Lorentz force equation [76]

$$
\frac{\partial \gamma m v}{\partial t}=q(\mathbf{E}+v \times \mathbf{B})
$$

where $m, q, v$ and $\gamma$ are the mass, charge, velocity of electron and the relativistic factor, respectively.

VSim can also model the random interactions between particles or the random production of particles. This uses a statistical approach called the Monte-Carlo collision model [65-67]. No particle to particle collisions are modeled nor are any particles created from collisions in this dissertation, so no discussion is provided on the Monte-Carlo model. The only interaction between particles is through the coulomb force between particles through the electromagnetic solver. 


\subsection{State of the Art in MVEDs}

\subsubsection{Cylindrical Emitting Sole CFAs}

The first cylindrical CFA was developed by William C Brown with Raytheon [77]. He called the device the Amplitron and it was based on the magnetron. It was basically a magnetron design (cylindrical reentrant design with a center thermionic cathode, and a strapped vane structure slow wave circuit) which was altered to accommodate an input and an output. After this demonstration, many configurations have been studied. A few examples are given in $[53,78,79]$.

Cylindrical, reentrant CFAs, which use an emitting sole cathode, are the most common CFAs in use today due to their compact size and the efficient recycling of unspent electrons. These conventional CFAs were explained in the beginning of Chapter 2. There are only a few published modern designs as many of the designs are proprietary and unpublished, but the general design is the cylindrical, reentrant and emitting sole CFA. The emitter materials vary for the application where the desired qualities of the emitting sole are a high secondary electron yield, good heat dissipation, and robust performance [2]. The desired qualities of the thermionic cathode is a low work function, high melting point, and long lifetimes [2]. No one to date, however, has published results showing the incorporation of GFEAs in a CFA. The qualities of the slow wave circuit that are important are bandwidth, coupling impedance, unwanted mode suppression, and power/heat dissipation. The slow wave structures also vary depending on the application. The most common slow wave structure in forward wave cylindrical CFAs is a double helix coupled vane shown in Fig. 2.26 [2]. This structure is made up of two helices and is supported by metal vanes. The metal vanes provide structure for the helices and also help dissipate heat. 


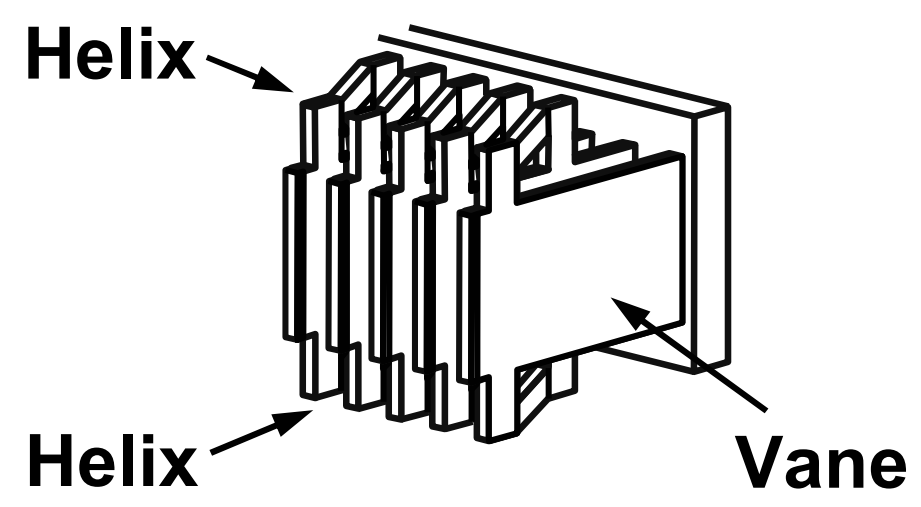

Figure 2.26: Double helix coupled vane slow wave structure commonly used in CFAs. [2].

\subsubsection{Power Capabilities}

The current power capabilities of CFAs are comparable to that in 1985, and a list of examples is shown in Fig. 2.27. Gain ranges from $7-20 \mathrm{~dB}$, efficiencies from $50-75 \%$, and bandwidths up to $10 \%$. Information on the exact design of these high power configurations is difficult to find. Generally, the only information available is the general figures of merit such as gain, output power, efficiency and noise level. A few examples without the design specifications in the lower frequency range $(450 \mathrm{MHz}-4 \mathrm{GHz})$, which is more relevant to the low frequency design proposed here, are found here [80-82]. The only thorough description of the design specifications found was of an X-band (11.424 GHz) crossed-field amplifier with an output of $300 \mathrm{MW}$ [83], which is described in the next section. 

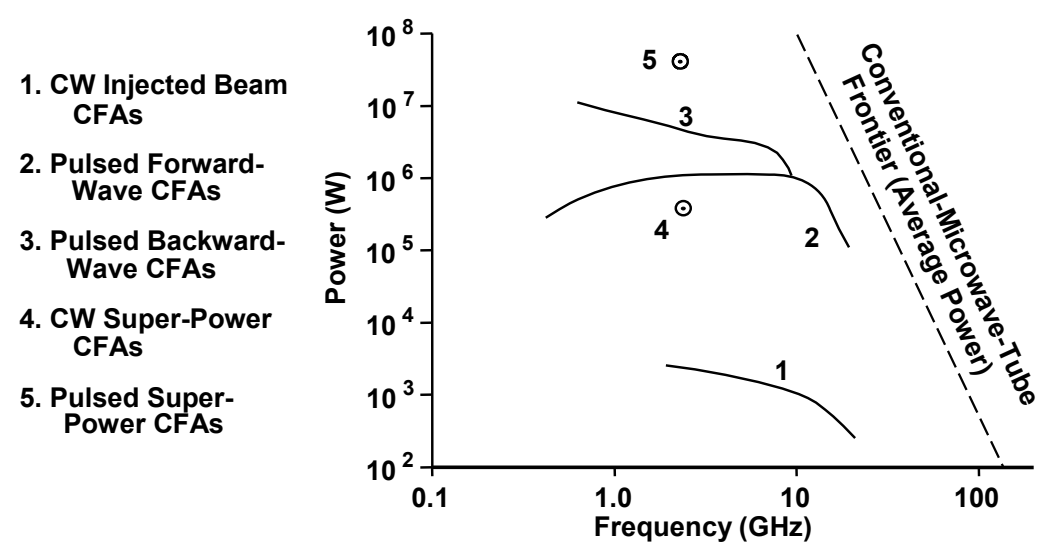

Figure 2.27: The current power capabilities of published CFA data. [2].

There are three factors that limit the output power of CFAs $[2,17]: 1)$ a limitation in the available cathode current, 2) the onset of a competing oscillation where the anode-to-sole voltage has reached a region of synchronism, or 3) a limitation in gain of the main amplifying mode when the RF drive power is no longer able to retain lock at the higher output power. The first two limitations can be avoided with appropriate design. Proper cathode design can increase the current to avoid the limitation set by factor 1. To prevent unwanted oscillation, proper junction matching techniques can be used to prevent reflections within the circuit, and selective attenuation techniques for the unwanted frequencies can be used $[7,17,84]$. The third limitation is an intrinsic limit resulting from the basic interaction process and is difficult to avoid. There are a few techniques to minimize the mode interference [7, 17], but mode interference remains the main limiting factor to gain and output power of CFAs.

\subsubsection{High Power X-Band Crossed Field Amplifier}

To demonstrate typical electron beam current densities and output powers, the specifications for a $11.424 \mathrm{GHz}$ CFA designed by Eppley et. al. [83] is presented here. The 
exact dimensions and description of the design is given in that work, but only a few characteristics are described here. This is a cylindrical format, cold cathode emitting sole, backward wave, reentrant CFA, similar to the one shown in Fig. 2.2 except this is a backward wave device. The design in this work was still in development at that time and presents no experimental results, but simulation results showed $300 \mathrm{MW}$ of output power at $65 \%$ efficiency with a RF drive power of $6 \mathrm{MW}$. The cathode current density was $41 \mathrm{~A} / \mathrm{cm}^{2}$, and $2600 \mathrm{~A}$ was observed at the anode. This current density is typical in CFAs, and this current density is currently achievable by GFEAs [25-27]. These operating characteristics will be used to explore GFEA use in high power devices in Sec. 7.8.

\subsubsection{Cathode Driven Crossed Field Amplifiers}

The cathode driven CFA $[85,86]$ has the basic format of the conventional cold cathode CFAs, but uses a slow wave circuit on the cathode as well as on the anode. This configuration decouples the output circuit from the input circuit when no electrons are present. The coupling occurs when electrons are present and an RF input signal is applied to the cathode circuit. Figure 2.28 shows 2 different variants of a cathode driven configuration with a comparison to conventional CFAs. Conventional CFAs, shown in Fig. 2.28(a), use a smooth cylinder to emit electrons. The RF electric fields from the anode are weakest at the cathode because it is radially disposed from the anode. The electron cloud at the cathode thus has a weaker frequency-determining component which produces a noise component, typically $50 \mathrm{~dB}$ below the output signal. By driving the RF at the cathode, the RF drive signal is highest at the cathode. Figure 2.28(b) shows the input signal on the cathode circuit alone. This showed an improvement to gain, but no improvement to noise. The importance of control over 
the entire electron trajectory is apparent from the cathode-driven only experiment, so a hybrid approach was developed. Figure 2.28(c) shows a hybrid approach, where the RF drive is applied to both the anode and cathode, providing improved electron trajectory control. The hybrid approach showed the gain improvement observed in the cathode-driven only experiment along with dramatic improvements to the signalto-noise ratio $(20 \mathrm{~dB} / \mathrm{MHz})$ over conventional CFAs.

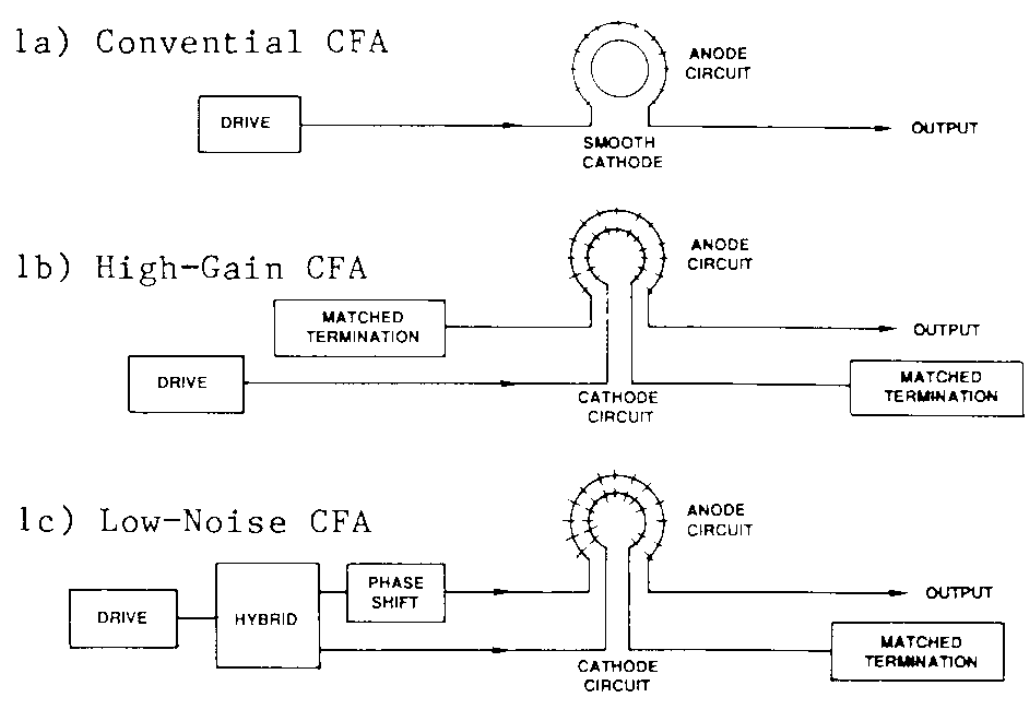

Figure 2.28: (a) conventional CFA comparison with a (b) cathode-driven and a (c) hybrid variant. [85].

This approach isolates the source power from load reflections. By decoupling the output from the input, the stability of the CFA is improved. This also extends its applications to ones where a variable load is used, such as particle accelerators [86].

\subsubsection{Linear Format Injected Beam CFAs}

A less commonly used CFA today is the linear format injected beam CFA. These CFAs were explained briefly in the beginning of Chapter 2. These devices use a variety of different slow wave circuits, but the general format is the same as described 
in the beginning of the chapter. The designs use a Kino type gun [87] to inject the beam current. Figure 2.29 shows both short and long type Kino guns. This gun design allows for dense electron beam in the presence of the magnetic field in CFAs. Another similarity in the linear injected beam CFAs is the use of depressed collectors $[7,88]$ to collect the electron beam at the end of the tube in order to increase efficiency. Depressed collectors are not discussed in this dissertation, but they increase efficiency of the device by essentially recycling leftover energy in the electron beam collected at the end of the tube and using it for beam emission.

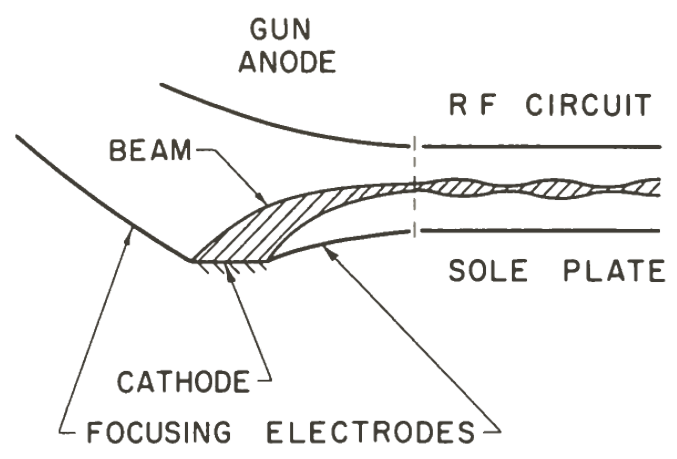

(a)

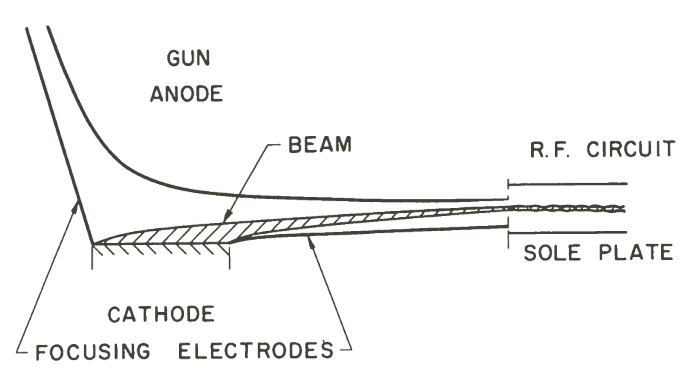

(b)

Figure 2.29: (a) Short and (b) long Kino electron gun schematics [87].

From the 1950s through the early 1970s much research was performed on these MVEDs because of the high efficiency of the interaction. These devices have since been replaced by TWTs because of their lower cost, higher gain, and greater stability [4]. Even though these MVEDs are not very common today, this is the type used in this dissertation, and a summary of them is given here. Many different Linear format injected beam CFAs have been tested [16, 84, 89-91], and a few important observations are noted here.

The exact description of the devices are not given here, but a summary of the 
general conclusions of those experiments is given. The maximum gain observed among these devices is in the $25-30 \mathrm{~dB}$ range $[84,90]$. Two general limits to getting high gain in these devices are (i) unwanted oscillations outside the matched band and (ii) beam noise. By using selective attenuation on the slow wave circuit and careful circuit termination, the oscillations can be suppressed. Selective attenuation introduces a frequency sensitive loss which attenuates unwanted signals and passes the designed frequency. Gilmour [2] discusses a few attenuation techniques to prevent backward wave oscillations in TWTs. Noise created by the beam is another limit to the gain of the device, and much of the noise is caused by the electron gun design and use of the thermionic cathode. A detailed discussion on noise reduction in CFAs is given by Gilgenbach et. al. [92].

The gain of linear injected beam devices is very sensitive to the beam injection technique $[19,91]$. The efficiency of the device depends largely on the cycloiding of the beam in the interaction space, which is highly dependent on beam injection. The experiments by Cooke and Döhler $[19,91]$ showed that by improving the electron gun optics and making the beam injection "smoother," significant gains to efficiency are observed.

Not only is the beam injection important, but the choice of the beam trajectory itself is important. The beam can be a Laminar flow type or a cycloidal type with varying cycloid radii. An article by Locke [16] developed a theoretical model to model highly cycloidal beams, compared it with experiments, and determined that CFAs which implement a highly cycloidal beam only require $35 \%$ of the interaction length of a laminar-beam type for the same output power, gain, and efficiency. The reason given for this improvement is that out-of-phase electrons which extract energy from the RF wave on the circuit are quickly removed from the device by the sole in 
a highly cycloidal trajectory. These out-of-phase electrons, if they remained in the interaction region, would continue to remove energy from the RF beam, but since

they are more easily removed from a highly cycloidal beam, less energy is removed. The research in this dissertation used the highly cycloidal beam.

\subsubsection{Linear Format CFA at Northeastern University}

The CFA described in this section was compared against the simulation results in this dissertation, so a detailed description is given here. In 1991, a group at Northeastern University in Boston, MA developed an injected beam linear format CFA which uses a $150 \mathrm{MHz}$ meandering microstrip line slow wave circuit $[14,15]$. This design is used in this dissertation and is shown in Fig. 2.30. The slow wave circuit was comprised of a meandering $1 / 8$ inch diameter copper tube placed on a $1 / 16$ inch thick Teflon dielectric which was placed on a copper ground plane. The circuit was $40 \mathrm{~cm}$ long and $25 \mathrm{~cm}$ wide with a $1 \mathrm{~cm}$ pitch. This slow wave design has a retardation of $R=33$. At the operating frequency of $150 \mathrm{MHz}$, the device length is only 6 slow wave wavelengths long. This length is rather short but is sufficient to see moderate gain. The sole to anode gap was $2.5 \mathrm{~cm}$.

Electrons are emitted from a $2 \%$ thoriated tungsten filament 10 mil in diameter, and the cathode generates an electron beam about $10 \mathrm{~cm}$ wide. A focusing electrode is used to inject electrons into the interaction region as shown in Fig. 2.30. The electron beam is highly cycloidal in order to maximize the interaction over short distances [14-16]. Many of the experiments use an electron beam current of $150 \mathrm{~mA}$. This current is used for most of VSim simulations for ease of comparison with experiment and ease of implementation due to the fact that this current is below the space charge limit of the configuration. 


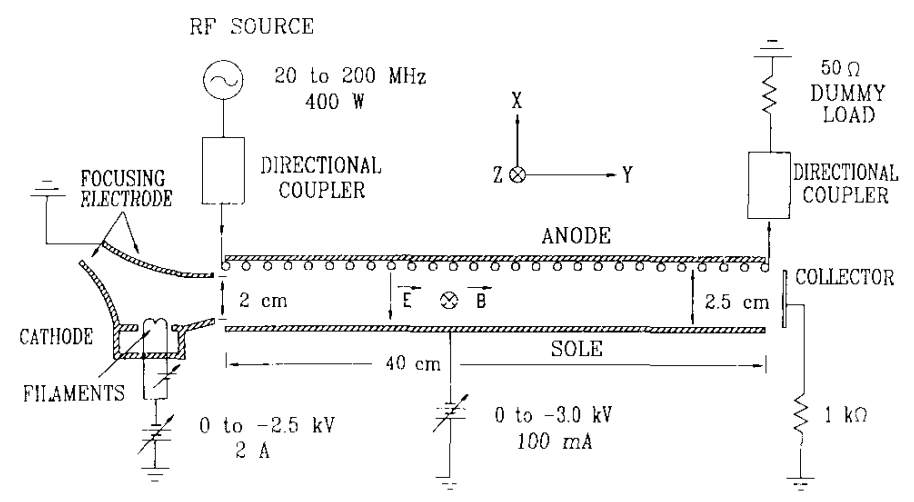

Figure 2.30: Northeastern CFA schematic in Browning et. al. [14, 15]

The goal of the Northeastern work was to implement in-situ measurements of the electron plasma inside the CFA interaction space during operation. This goal resulted in a low frequency CFA so that the interaction region and RF wavelength were large enough to allow diagnostic probes to be used. Measurements were performed for RF power vs. device length, electron density vs. device length, electron energy distribution of the beam, bandwidth, gain vs. electron beam current, and Langmuir probe current. The measurements of gain vs. frequency and gain vs. beam current are shown in Figs. 2.31 and 2.32, respectively. These two plots will be compared against the VSim results to validate the model. 


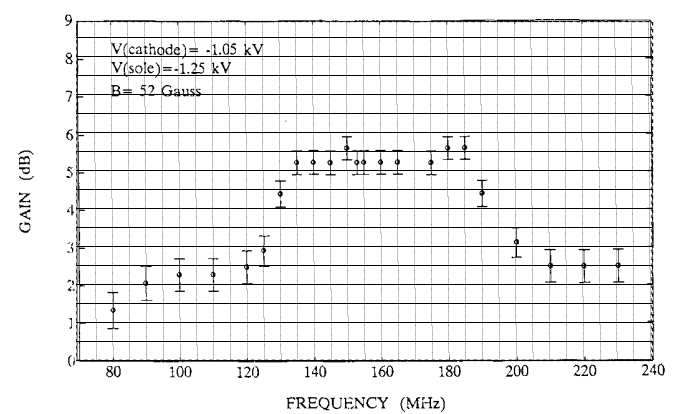

Figure 2.31: Northeastern CFA Gain vs. frequency plot in Browning et. al. $V_{a s}=1250 \mathrm{~V}, B=$ $5.2 \mathrm{mT}[14]$

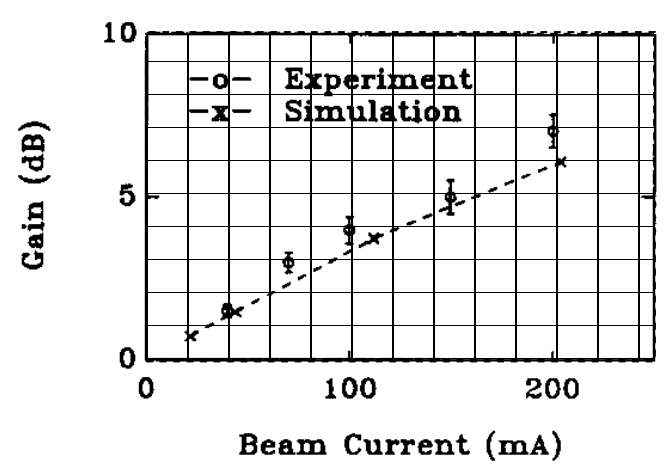

Figure 2.32: Northeastern CFA Gain vs. Beam current plot in Browning et. al. $V_{A S}=1200 \mathrm{~V}$, $B=5.5 \mathrm{mT}[\mathbf{1 5}]$

Another relevant result in this work is the measurements of RF power vs. device length. Fig. 2.33 shows the RF power near the circuit along the length with and without an electron beam. Without the beam, a standing wave pattern emerges whose amplitude fluctuates between 5 and 1 for the entire length. With the beam, a standing wave is also present, but with an increase in amplitude from 0 to $20 \mathrm{~cm}$ and then a constant amplitude from then on. From this result, it was concluded that the gain occurs within the $10-20 \mathrm{~cm}$ length and not the rest of the circuit. This result that the gain occurs only in the first portion of the circuit, if it is true, helps motivate the use of a distributed cathode. Because the gain saturates over relatively short distances, more gain can be achieved by injecting more current after this point. And, in general, the current can be tailored in such a way to help control the gain down the length of the circuit. It should be noted, however, that the RF field is found not to be a good indication of the gain along the length of the circuit based on a simulation study later in this dissertation. 


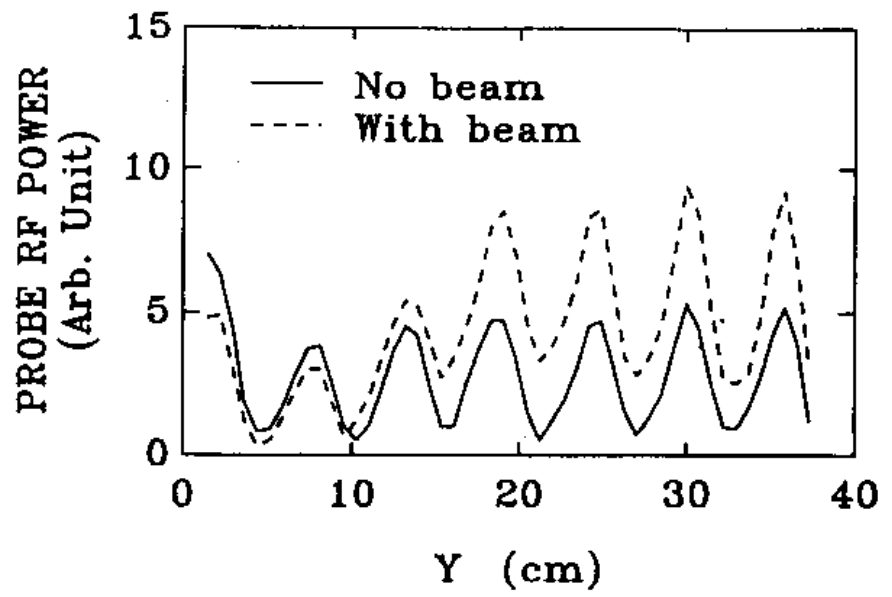

Figure 2.33: Northeastern CFA Gain vs. circuit with and without an electron beam in Browning et. al. $P_{r f}=10 \mathrm{~W}, V_{A S}=1200 \mathrm{~V}, B=5.5 \mathrm{mT}[15]$

\subsubsection{Simulation of a Distributed Cathode in a Rising Sun Magnetron}

This ongoing research [13, 93-95] focuses on simulation of a rising sun magnetron with a controllable distributed cathode. That work proposed to use gated field emitters as the cathode instead of a thermionic cathode. To test the benefits of using field emitters, simulations were performed using a controllable cathode source. By modulating the injected current to control the spokes in the magnetron device, improved startup times and efficiency were observed and showed reliable dynamic phase control [94]. Startup times were improved from $100 \mathrm{~ns}$ for the continuous current case to $40 \mathrm{~ns}$ for the modulated cathode case. Efficiencies in the work are not considered the absolute efficiency of the device, but relative comparisons can be made and the efficiency was improved from $80 \%$ for continuous current to $95 \%$ for modulated current. The work also showed reliable and efficient phase control of the oscillations. And the work showed that the phase can be actively controlled even after the device was oscillating. By shifting the phase of the modulated cathode emission, 
the spoke locations can be controlled, which controls the phase of the output. The work also determined that only $20 \%$ of the total current needs to be modulated in order to get the majority of the benefit to startup time, efficiency, and phase control[95]. Magnetrons are very similar to CFAs, and the promising results in that work indicate that the CFA will benefit from GFEA integration as well.

\subsubsection{Field Emitter Use in Microwave Vacuum Electron Devices}

The use of FEAs in MVEDs has been proposed and implemented for microtriodes [5, 96, 97], klystrodes [6, 32, 96, 97], twystrodes [96], gyrotrons [20], magnetrons [12, 28, 29, 98], and TWTs $[8,9,99,100]$. The use of GFEAs in gated emission devices such as the microtriode, klystrode, and twystrode is very appealing due to the low transconductance (the ratio of the change in current at the output terminal to the change in the voltage at the input terminal of an active device), short transit times (the time for an electron to travel from the emitter to the gate), and the small package. This approach would allow for high gain, high frequency, small devices. The advantages of GFEAs in TWTs, aside from the improvements to size and efficiency, is the ability to pre-bunch the beam before entering the interaction region. These "emission gated TWTs" can greatly improve the RF performance.

\subsubsection{FEA Use in CFAs}

No one to date has published experimental results utilizing FEAs in CFAs, but there has been, however, some theoretical work done by Sokolov et. al. $[18,96]$ with a distributed FEA cathode in a microelectronic CFA. In that work, the interaction

space had to be quite long (tens of wavelengths) in order to accommodate the use of a FEA cathode. This is a disadvantage since the losses in microelectronic lines are 
much greater. The focus in that work was about the use of two delay line structures to minimize losses in the delay line. The use of two delay lines showed $7 \mathrm{~dB}$ improvement over just using one long delay line.

\subsubsection{FEA Use In TWTs}

The TWT work by Whaley et. al. [8,9] is the only published work of a manufactured forward wave device which merits a short discussion. The general design of the TWT remains unchanged from standard TWT designs with only the addition of a GFEA. The main differences are in the beam injection region. Special design considerations were implemented to focus the beam and to protect the GFEA from ion back bombardment. GFEAs have significant beam spread due to the lack of focusing on the emitter tip and space charge defocussing, and the TWT requires good focusing to be optimum. Also, the GFEA has the ability to have independent control over current, decoupled from the accelerating voltage. The focusing technique must be able to work over a wide range of beam currents and acceleration voltages to realize the full potential of GFEAs. Ion back bombardment will degrade the performance of the GFEA and limit the lifetime of the device; therefore protection is necessary.

To properly modulate the cathode, a resonant matching circuit, based on a design from Calame et. al. [97], was implemented and is shown in Fig. 2.34. This circuit reduces the necessary RF drive power of the GFEAs, and also allows for a way to maintain the bandwidth of the device. The drive power in this circuit when used as a resonant matching system is given by Eq. (2.28), where $C_{\text {eff }}$ is the GFEA capacitance, $\omega$ is the drive frequency in $\mathrm{rad} / \mathrm{s}, V_{0}$ is the $\mathrm{RF}$ amplitude of the signal, and $Q_{t o t}$ is the total quality factor of the system. For higher bandwidth applications, the drive 
power of the non-resonant design is given in Eq. (2.29), where $R_{\text {eff }}$ is the effective resistance of the GFEA and because $R_{e f f}$ and $C_{\text {eff }}$ are very small, $\omega R_{e f f} C_{\text {eff }} \ll 1$.

$$
\begin{gathered}
P_{r f}=\omega C_{e f f} V_{0}^{2} / Q_{t o t} \\
P_{r f}=\frac{1}{4} \omega C_{e f f}\left[\omega R_{e f f} C_{e f f}+\sqrt{1+\left(\omega R_{e f f} C_{e f f}\right)^{2}}\right] V_{0}^{2}
\end{gathered}
$$

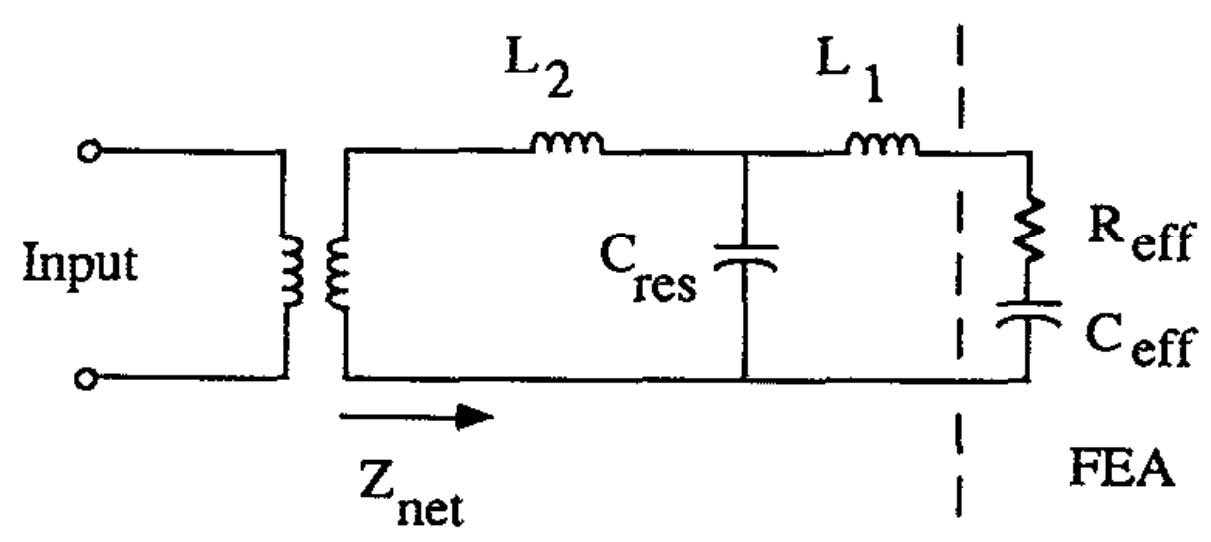

Figure 2.34: GFEA matching circuit used in the TWT work [8], proposed by Calame [97]

Whaley et al. [8, 9] have successfully created and operated a $100 \mathrm{~W}$ TWT with the use of GFEAs. They developed a new way to focus the beam from GFEAs using multiple lenses. They also implemented a ion shield using a region of positive potential relative to the system between the cathode and the interaction region. They were successful in implementing a $100 \mathrm{~W}, 5 \mathrm{GHz}$ TWT with a small signal gain of $32.7 \mathrm{~dB}$, a saturated gain of $22.1 \mathrm{~dB}$, and a circuit efficiency of $24 \%$. Life tests of this device were rather short with $150 \mathrm{~h}$ of cumulative pulsed operation. 


\subsubsection{FEA use in Magnetrons}

There has been ungated FEA work performed on relativistic magnetrons [29, 101]. Relativistic magnetrons use explosive emission [30, 31] for the electron emission. Explosive emission is a phenomenon observed when a field electron emitter explodes due to very high current density. By using very high voltage pulses $(>500 \mathrm{kV})$ enormous amounts of currents can be observed $(>5-10 \mathrm{kA})$ from explosive emitters [28]. In relativistic magnetrons, gigawatts of pulsed power can be observed at efficiencies of 20-40\%. Many different types of cathodes have been developed and studied [102-106] with a focus on the minimization of plasma formation.

The most relevant work to the distributed cathode research in this dissertation is the transparent cathode work $[11,12,98]$. Figure 2.35 shows the transparent cathode configuration in an A6 magnetron [98]. Instead of using a solid cathode as the electron source, a series of six cathode strips are used. Instead of a uniform current originating from a center solid cathode, there are six discrete sources of electrons. The transparent cathode has two main advantages: the azimuthally modulated electron emission (cathode priming) shortens the rise time of power generation and the absence of the solid core allows strong azimuthal electric fields near the cathode, improving the electron beam- RF wave interaction [107]. The number and position of the cathode strips was found to affect the mode and operation of the device. With the number of strips equal to half of the cavities, the $\pi$ mode grows rapidly; when the number of strips is equal to the number of cavities, the $2 \pi$ mode is excited. Varying the angle found a difference of $90 \%$ of the maximum output power from optimum and unfavorable positions. 

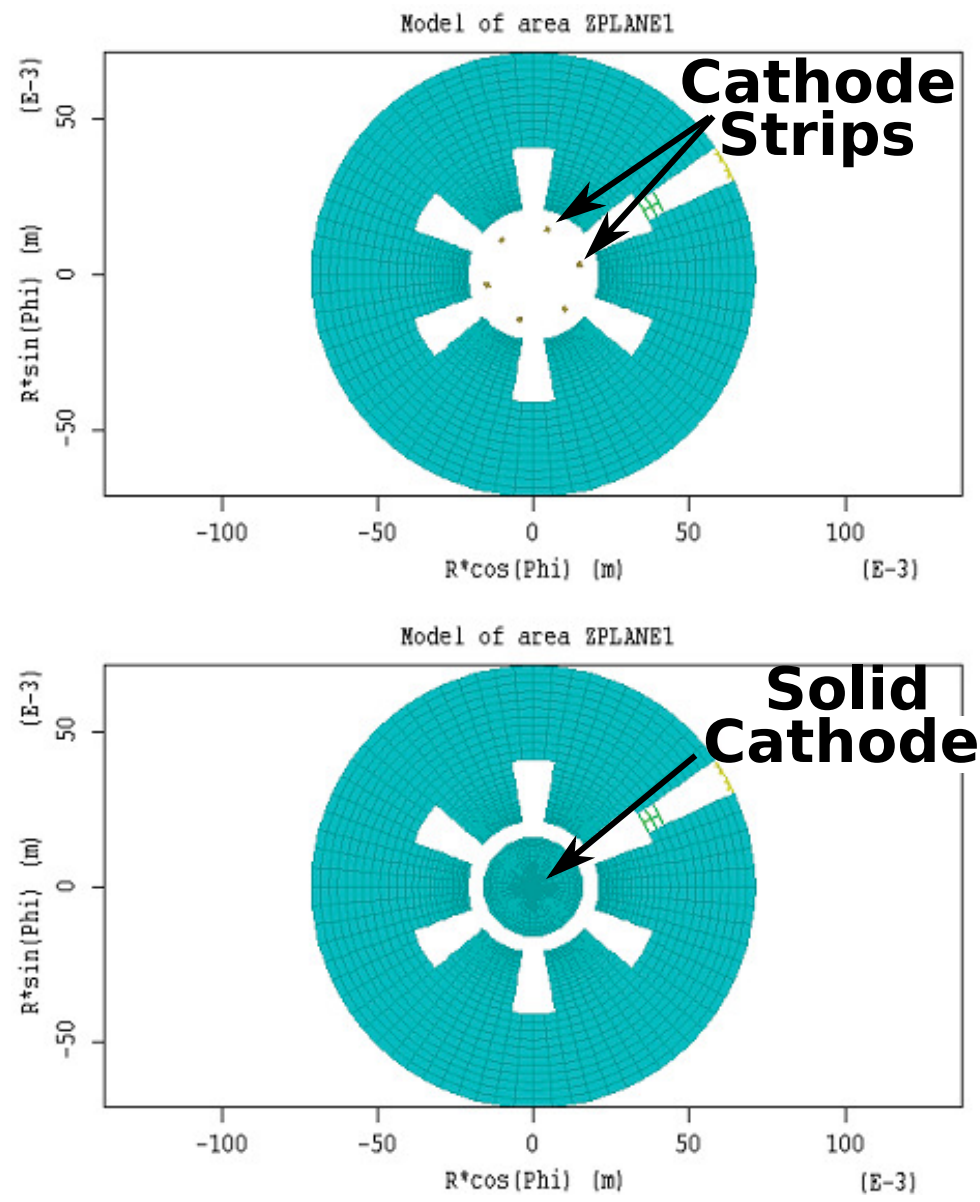

Figure 2.35: (top) The transparent cathode configuration with 6 cathode strips and (bottom) a solid cathode [98]. 


\section{CHAPTER 3}

\section{RESEARCH OVERVIEW}

The goal of the research is to study new CFA designs which use a controllable, distributed cathode to tailor the electron current injection to improve gain, efficiency, and noise. Originally, experiments and simulations using VSim [42] were proposed to study the effects of the electron current profiles from a distributed cathode, but the experimental design showed no electron beam interaction with the RF wave on the circuit. An extensive investigation of the electron beam trajectories and the dispersion characteristics of the slow wave circuit ruled out these as the source of the

problem. It was determined that the maximum current available cathode was less than the minimum current needed for interaction. Because of this, the research focus was shifted to a simulation of a CFA design studied at NU [14, 15]. Two different CFA designs were tested experimentally here at BSU, and three different designs were studied via simulation. This chapter presents the proposed experimental design and outlines the chronology of research.

\subsection{Proposed Experimental Design}

The proposed CFA design is a linear format with a meander microstrip line for the slow wave circuit. GFEAs in conjunction with hop funnels were proposed to implement the controllable distributed cathode. GFEAs provide a simple way to have a controllable 
distributed cathode. Hop funnels provide the protection for the GFEAs from the high electric fields and current densities of the interaction region and provide a way to control the energies of the injected electrons separately from the sole potential. Two different configurations are proposed in this work: an injected beam and a distributed beam.

\subsubsection{Injected Beam Configuration Experiment}

The injected beam CFA pictorial schematic is shown in Fig. 3.1 along with the dimensions. The electron trajectory is shown as the red cycloidal line. The electrons are emitted from the GFEA and follow the cycloidal trajectory due to the crossed magnetic and electric field. The cycloidal trajectory in the figure is a pictorial representation and not representative of the actual trajectory. The electrons enter the interaction region, interact with the RF wave on the circuit, and collect either on the slow wave circuit or the end collector. The electric field in the interaction region is controlled by the potentials on the sole and slow wave circuit. The magnetic field is controlled by external Helmholtz configuration. An RF wave is input on the slow wave circuit on the left, and if the electron velocity is close to the phase velocity in the interaction region, the $\mathrm{RF}$ wave will be amplified at the $\mathrm{RF}$ output on the right. Note that the GFEA cathode is below the sole electrode in some parts; this is because the GFEAs available to the group were large $(9.5 \times 12.5 \mathrm{~cm})$, and this was the best way to fit the cathode in the CFA chamber. The GFEA cathode and slow wave circuit are discussed in the next sections. 


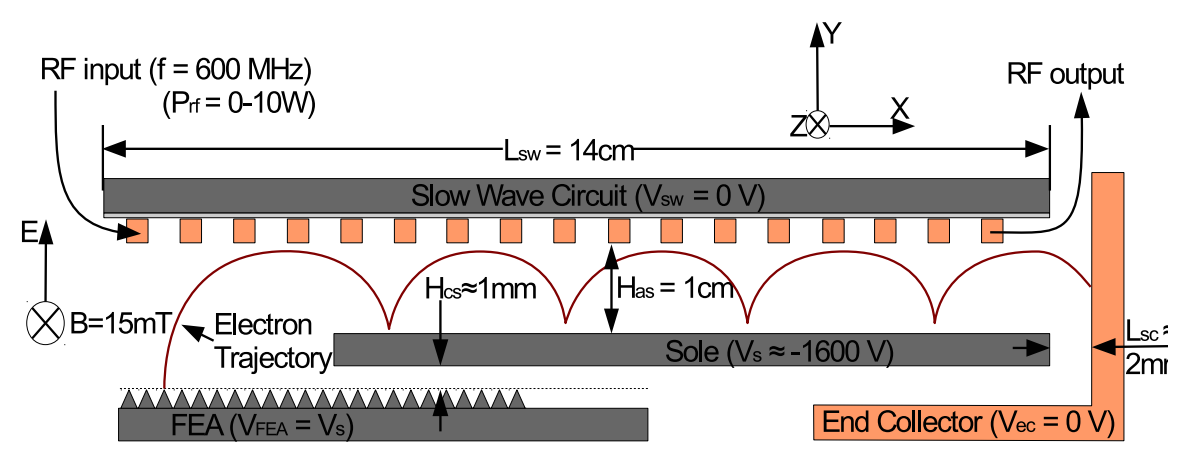

Figure 3.1: Schematic representation of the injected beam CFA design with dimensions, not to scale.

\subsubsection{Meander Line}

A meander line microstrip circuit is used as the slow wave circuit. The meander line circuit is used because of its ease of manufacture and ease of impedance matching. The practical use of meander lines is limited by the inability of the circuit to dissipate power and by dielectric charging. Because of the lower power operation of this CFA, the meander line is sufficient.

Figure 3.2 shows the geometry and dimensions of a generic meandering microstrip circuit. The exact dimensions and parameters of operation of the circuits used in this work are listed in Table 3.1. The circuits were designed to be at least 6 slow wave wavelengths long and to fit in the chamber available to our group. Two circuits were designed and used. The first circuit, SW1, experimentally demonstrated undesirable phase velocities, so a new design, SW2, was developed. Much of the results are redundant between the circuits, so the experimental focus is on the circuit called SW2. 


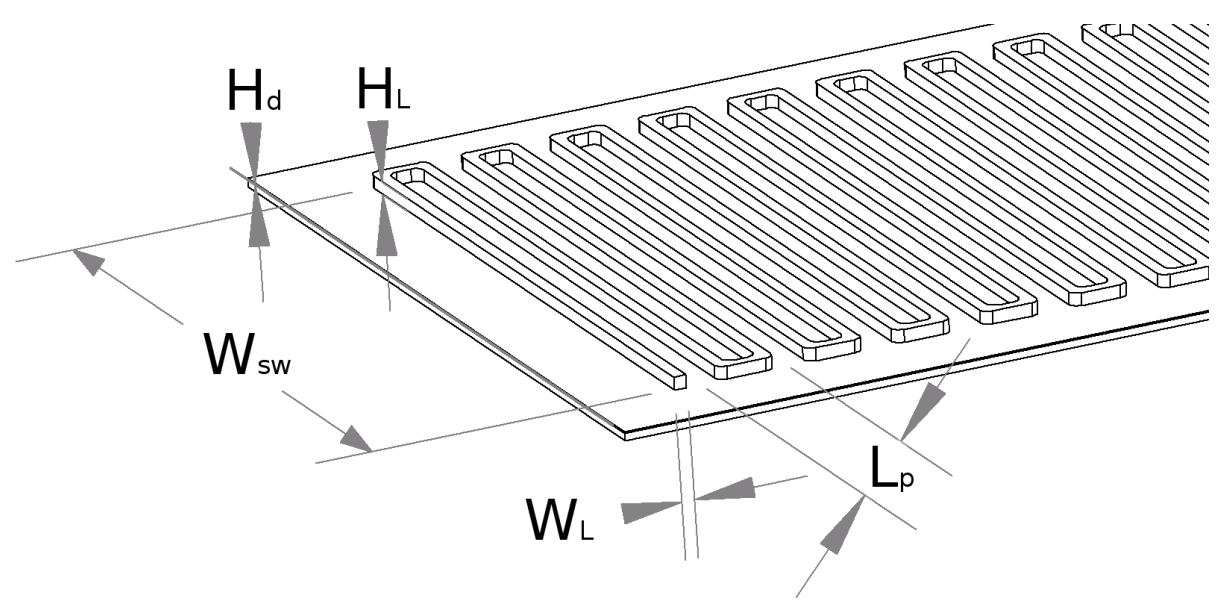

Figure 3.2: The diagram showing the meander line microstrip. A metal line meanders over a dielectric with thickness $H_{d}$ over a ground plane.

Table 3.1: Slow wave specifications

\begin{tabular}{|c|c|c|c|c|c|c|c|c|}
\hline Name & $\begin{array}{c}\text { Period } \\
(\mathrm{P}) \\
{[\mathrm{mm}]}\end{array}$ & $\begin{array}{l}\text { Width } \\
(\mathrm{W}) \\
{[\mathrm{mm}]}\end{array}$ & $\begin{array}{l}\text { Line } \\
\text { Width } \\
{[\mathrm{mm}]}\end{array}$ & $\begin{array}{l}\text { Line } \\
\text { Height } \\
{[\mathrm{mm}]}\end{array}$ & $\begin{array}{c}\text { Dielectric } \\
\text { Thickness } \\
\text { [mm] }\end{array}$ & $\begin{array}{c}\text { Effective } \\
\text { dielectric } \\
\left(\epsilon_{r}\right)\end{array}$ & $\begin{array}{c}\text { Estimated } \\
\text { Retardation } \\
\text { (R) }\end{array}$ & $\begin{array}{c}\text { Operating } \\
\text { Frequency } \\
{[\mathrm{MHz}]}\end{array}$ \\
\hline SW1 & 8 & 50 & 1.5 & 1.8 & 0.5 & 1.796 & 18.09 & $800-1000$ \\
\hline SW2 & 7 & 74 & 1.2 & 1.8 & 0.33 & 1.815 & 29.83 & $400-600$ \\
\hline
\end{tabular}

\subsubsection{Cathode}

The proposed cathode for this CFA is a GFEA. The GFEA available to the group was a Spindt type gated field emitter array [23] obtained from PixTech Field Emission Displays fabricated in 2001 [108]. The cathode unit was $9.5 \times 12.5 \mathrm{~cm}$ and the CFA configuration was designed around this constraint. The emission area is about $4 \mathrm{~cm}^{2}$, and the desired current was on the order of $100-200 \mathrm{~mA}$. GFEAs at the time this CFA was designed (2011) had demonstrated current densities of $20 \mathrm{~A} / \mathrm{cm}^{2}$, which would theoretically allow for $80 \mathrm{~A}$ of current from the emission area, but space charge 
limits the current with the electric fields used in the CFA to currents on the order of $100-200 \mathrm{~mA}$. At the time of this writing, current densities of $100 \mathrm{~A} / \mathrm{cm}^{2}$ have been achieved by GFEAs developed by Guerra et. al. at MIT [25-27].

\subsubsection{Distributed Cathode}

The distributed cathode configuration includes the same meander line circuit and electron source as the injected beam configuration. The difference is the sole design. The distributed cathode CFA pictorial schematic is shown in Fig. 3.3. In this configuration, electrons are emitted up into the hop funnel, 'hop' up the dielectric wall, and enter the interaction region. There are multiple injection points in this design, and electron current at each injection point can be controlled by the GFEA. The potentials between the sole electrode and the slow wave circuit control the electric field in the interaction region while the hop electrode controls the electron energy of the electrons.

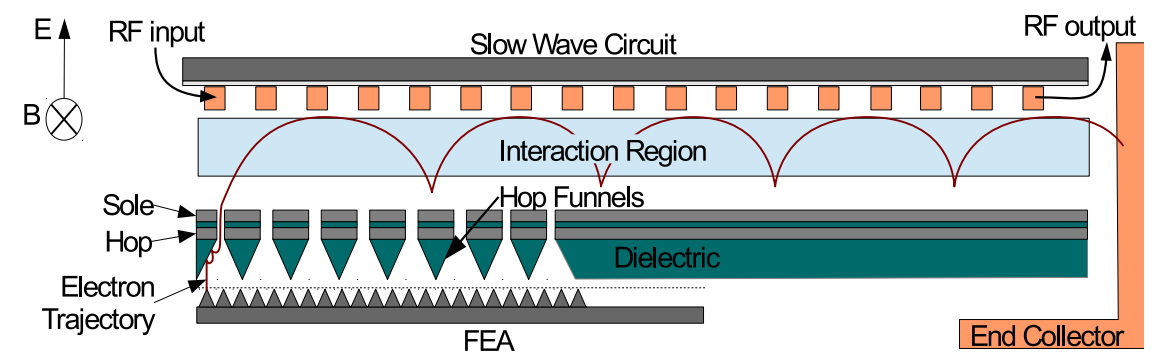

Figure 3.3: Schematic representation of the distributed cathode CFA design, not to scale. Electrons injected into the hop funnels are extracted though slits in the sole electrode. 


\subsubsection{Sole/Hop Funnels}

The hop funnels were fabricated out of Low Temperature Co-Fired Ceramic (LTCC) [109]. A schematic of the hop funnels/sole is included in Fig. 3.3. The LTCC spans the width and length of the interaction region. Two layers of metal are on the surface of the LTCC structure separated by a dielectric layer. The two metal layers are called the hop and sole electrodes. As explained in Chapter 2, the hop electrode is used to control the energy at which the electrons are born, and the sole electrode is biased more negative than the hop electrode in order to prevent cycloidal electrons from collecting on the sole.

\subsection{Research Chronology}

Because no gain was observed experimentally, the focus of the work shifted to simulation of a design used by a group at Northeastern University [14, 15], which is very similar to the injected beam configuration presented here. The Northeastern design and the simulation model are explained later. Even though the proposed designs showed no gain, valuable data was still obtained for comparison to the simulation for validation. The experimental injected beam design is the simplest control variation to easily test the general function of the CFA. The distributed beam configuration was briefly tested by the group but no results are presented from that experiment.

Three different linear format CFA designs are studied in this dissertation. Two were developed at BSU for this dissertation (CFA1 and CFA2 which use slow wave circuits SW1 and SW2, respectively), described above, and one was designed at Northeastern University $[14,15]$ to perform in situ measurements of the interaction region. Each of the designs contribute to the research but the main contributions 
come from simulation of a distributed cathode variant of the NU CFA design. This section describes the research flow and major mile markers of the research. Figure 3.4 shows a visual diagram of the research flow. 


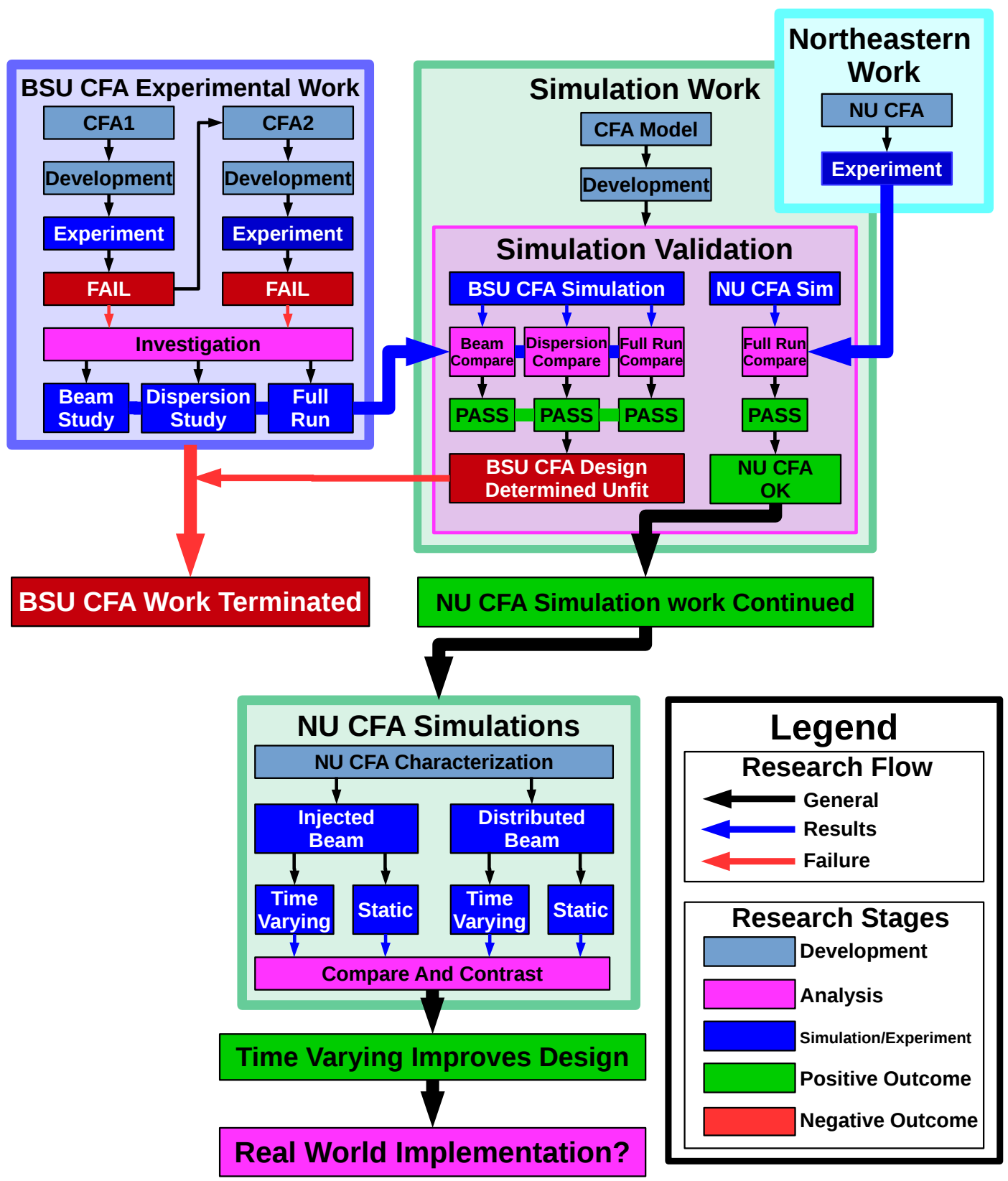

Figure 3.4: Diagram outlining the research flow of the three CFA designs. The BSU experimental work was used to validate the simulation model, but all work on the BSU CFAs were terminated after determining the design was unfit. Results from the Northeastern CFA experimental work were also used to validate the simulation model, and the design was used for the distributed cathode studies. 
The original goal of this research was to experimentally and computationally study the effects of different emission profiles using a fully controllable distributed beam in a CFA. The first two designs, CFA1 and CFA2 which use slow wave circuits called SW1 and SW2, respectively, were developed at BSU for this purpose. Injected beam CFA experiments showed no RF interaction with the beam. Three different possibilities were investigated to determine the problem: slow wave circuit dispersion, electron beam trajectory, and insufficient electron beam current. Dispersion measurements were performed and compared with COMSOL and VSim simulations. Dispersion measurements showed a higher phase velocity than predicted from simulation but corroborated the general behavior and trends.

The available equipment prevented matching the electron beam $\mathbf{E} \times \mathbf{B}$ velocity to the high phase velocity of SW1, which instigated the development of SW2 used in CFA2. Measurements concluded that SW2 also had a higher phase velocity than predicted from simulation, but allowed for a testable CFA setup with the available equipment. Still no RF wave electron beam interaction was observed.

A thorough investigation to determine the reason for the lack of RF wave interaction with the electron beam showed that much more current was required than was capable of the GFEAs used in the experiment. VSim modeling and analytical analysis using Pierce theory both showed a current of $150 \mathrm{~mA}$ is needed to observe appreciable gain where only $5 \mathrm{~mA}$ of current was available from the PixTech cathodes. The BSU CFAs would never show RF interaction with the electron beam with the available equipment, so the design was shifted to the experimentally verified design from NU. The notable milestones in the BSU CFA experiments and simulations were dispersion experimental validation of the VSim model, simulated confirmation of experimental problem with the design, and the decision to shift to a simulation focus of the NU 
CFA design.

The next phase in the research was the experimental validation of the RF wave interaction with the electron beam in VSim using the NU CFA. VSim simulations were performed to model the exact operating parameters of the NU CFA design. VSim results matched the NU experimental results rather well, and it was determined that VSim model using the NU CFA design was a viable method to test a distributed cathode.

After the model was validated against experiments, the focus shifted to characterization of the various cathode implementations. Four different methods were tested: static injected beam, modulated injected beam, static distributed beam, and a modulated distributed beam. Each of these methods was compared, and it was determined that the time varying methods improved the design. This is expected and compelling, but the impact of this result is questionable without a viable method to implement the GFEAs in high power devices. The discussion then shifts to implementation ideas. 


\section{CHAPTER 4}

\section{CFA EXPERIMENTS AND MEASUREMENTS}

This chapter describes the experiments performed on SW1 and SW2. The full CFA setup with these circuits never showed any gain, but a brief description is given here. Experiments characterizing the electron beam and slow wave circuit dispersion were successful and used to validate the VSim simulation model, so a more detailed description is given for those setups.

\subsection{Full CFA Setup}

The vacuum chamber, electromagnets, the CFA Structure, measurement hardware and LabVIEW software has been built and tested. A summary of the setup is given here.

\subsubsection{Vacuum Chamber and Electromagnets}

The chamber system is shown in Fig. 4.1. The electromagnets surround the chamber and allow for a nine inch sphere of uniform magnetic field inside the chamber. The CFA fits inside this sphere. The pressure of the chamber during operation was in the $10^{-7}$ Torr range. 


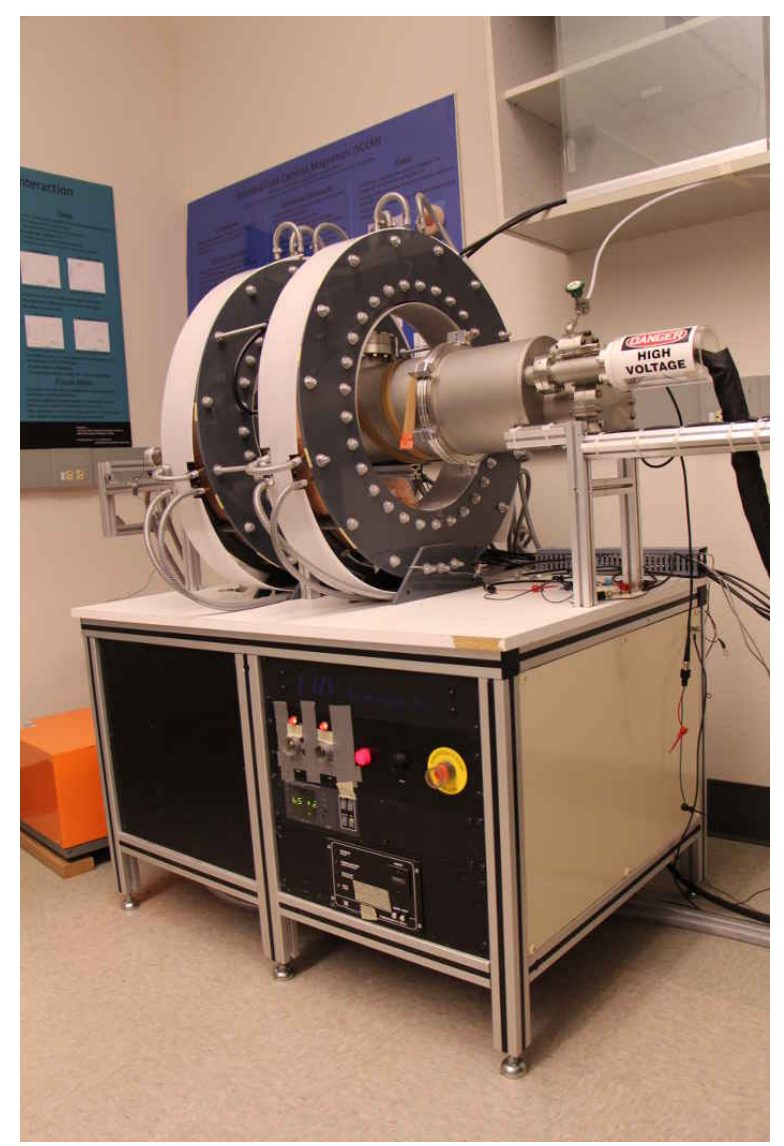

Figure 4.1: Photograph of the electromagnets and the chamber system where the CFA experiments are run.

\subsubsection{CFA Structure}

The experimental CFA pictorial schematic is shown in Fig. 4.2. The resistors in the schematic are to measure current to the electrodes and to limit arcs. These resistors are fixed resistors, sized so that the voltage drop is well above the noise level and within the range of the analog to digital converters $(\sim 1 \mathrm{~V}$ drop at the expected current, $1 \mathrm{k} \Omega$ to $1 \mathrm{M} \Omega$ at $1 \mathrm{~mA}$ to $1 \mu \mathrm{A}$ currents). The Interaction region is the gray region in between the sole and the slow wave circuit. This region is where the electrons interact with the RF wave. The end hats in the schematic are shown as dashed lines 
to represent that they are not in the interaction region, but they bound the region on both sides in the z-direction. These electrodes help contain the electron beam within the interaction region.

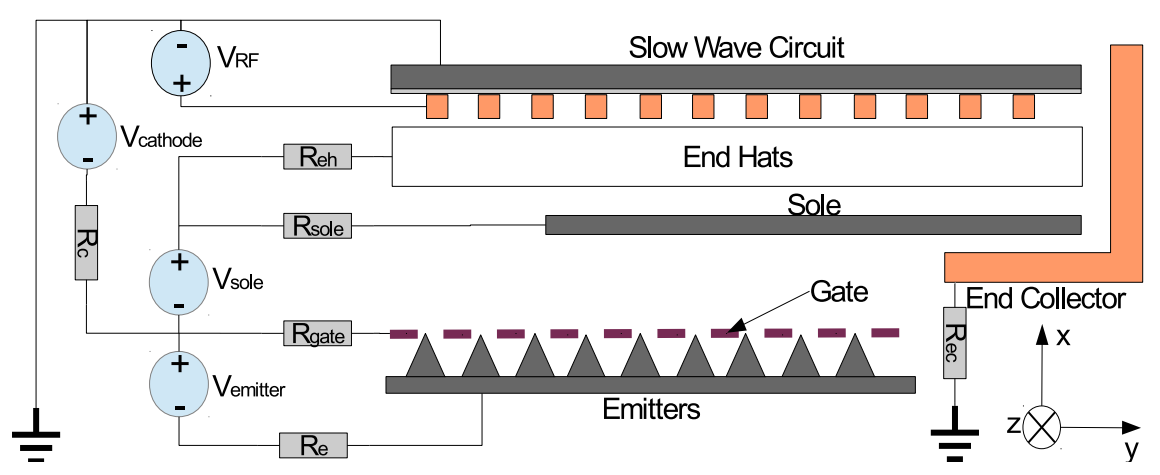

\section{Figure 4.2: Schematic representation of the CFA design, not drawn to proportion.}

The reason the GFEA extends below the sole electrode is due to the size of the GFEAs available. Section 4.1.4 describes the PixTech cathodes in detail. The CFA design must accommodate this large cathode structure. Fig. 4.3 shows a photograph of the CFA structure without the slow wave circuit. The slow wave circuit in this figure would sit over the sole, overlapping the end hats a little bit. Electrons are emitted from the GFEA and cycloid down, in this view, between the sole and the slow wave circuit. The end hats prevent the electrons from escaping out the sides of the device. To control the emission of the GFEA, Kapton coated wire is fixed to the PixTech cathode by a combination of silver paste and tape and is labeled gate connection in the figure. 


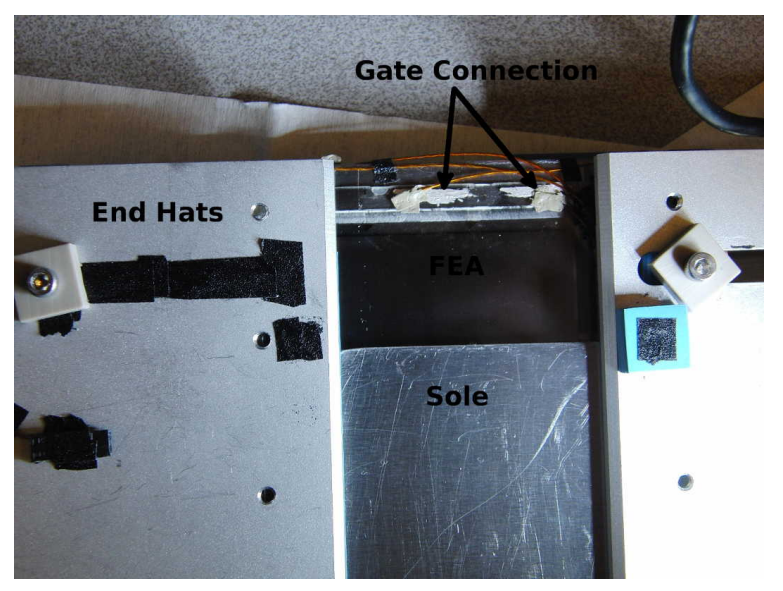

Figure 4.3: Top down view of the CFA structure without the slow wave circuit.

\subsubsection{Slow Wave Circuit}

Two meander lines were built and studied. The dimensions of each circuit are provided in Chapter 3. Fig. 3 shows one of the two circuits, SW2, built in this work. The circuit is a type of microstrip that meanders at $90^{\circ}$ angles over a Teflon dielectric which is over a conducting ground plane. Note that the wire height is fairly large for a strip line. This is to minimize the charging of the dielectric during amplification in the CFA configuration. Any electrons that manage to come close to the slow wave structure will be scrapped off by the protruding wire as opposed to striking the Teflon.

Of course, with high RF power and amplifications, microstrip slow wave structures encounter high electron bombardment. The meander slow wave structure will most likely not be able to handle high electron bombardment, and this is one limiting factor of the maximum power output. 


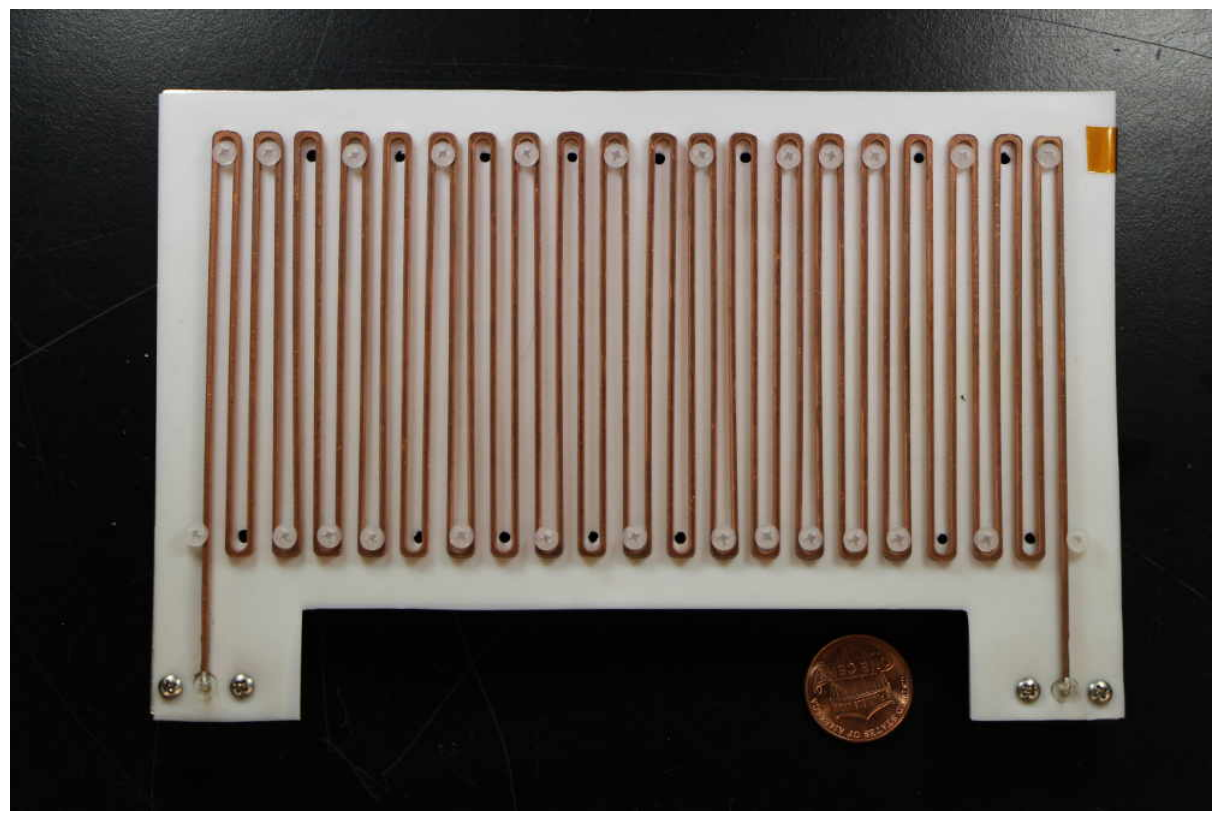

Figure 4.4: Photograph of slow wave circuit SW2. A rectangular copper wire meanders on top of a Teflon dielectric which is on top of an aluminum ground plane. The copper wire is fixed to the ground plane by polypropylene screws. The input an output ports are SMA connectors which are connected to the copper wire by silver paste.

\subsubsection{GFEA}

The main reason the experiment never showed any electron beam interaction with the RF wave was due to the inability of the cathode to supply the necessary current. This section describes the cathode itself, the various problems encountered when using the cathodes, and the reason for the low obtainable current.

The electron source used in this research is a Spindt type gated field emitter array [23] obtained from PixTech Field Emission Displays fabricated in 2001 [108]. To remove the cathode from the display assembly, the glass frit seal was broken. Many of the cathodes were damaged during the removal process. Many of the cathodes used in the experiment were cracked from disassembly. The main side effect of a cracked 
cathode is an increase in electrical short circuits between the gate and the emitter tip which increases leakage current. The increased leakage current limits continuous operation time due to ohmic heating and limits the cathode current control. To limit the leakage current, all the gates and emitters not used were left floating. Ideally these unused sections would be reversed biased to prevent current emission. Because of the complicated network of shorts between gate and emitters a various locations of the cathode, some parts of the cathode would be forward biased due to forward biasing of the active section. From this, unwanted emission sites were active and had to be accounted for in the CFA design. To prevent unwanted current from entering the interaction region or damaging components, usually a piece of metal was placed to intercept the current.

Figure 4.5 shows an example of one of the PixTech cathodes laid out on the CFA platform. Notice the crack on the bottom left corner. This is caused by the disassembly process. The lighter color streaked portion on the left side of the cathode is damage caused by arcing when operating the CFA in preliminary work. These preliminary experiments experienced many arcs and damaged many cathodes. The current CFA configuration prevents cathode damage. The gate connections are on the top edge of the cathode, and the emitter connections are along the left edge. the majority of the cathode sits under the sole, the metallic sole platform, and the end hats except for the emitting portion, as shown in Fig. 4.3. 


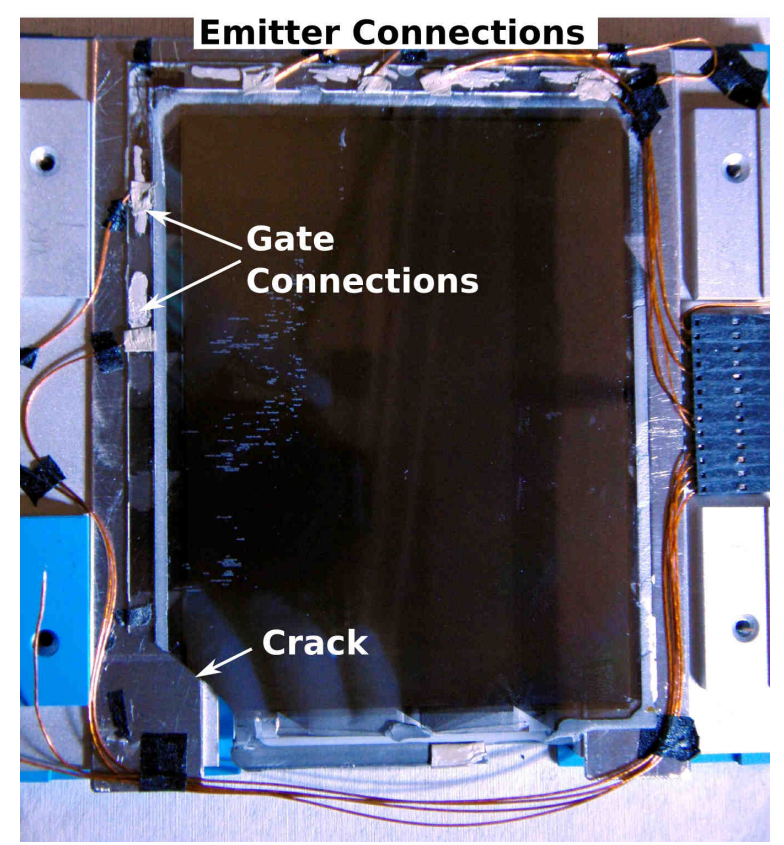

Figure 4.5: Top down view of the CFA structure without the slow wave circuit and the end hats to show the PixTech cathode and the gate and emitter connections.

\subsection{Meander Line Dispersion Measurements}

To determine the phase velocity of the circuits, some experiments were performed. These measurements are compared with dispersion measurements simulated in COMSOL and Vsim. The experimental setup is outlined here.

\subsubsection{Experimental}

Two different experiments were performed on the meander lines to determine the dispersion characteristics: (1) measure the standing wave pattern and (2) determine the S-parameters using a Network analyzer. The main goal of these experiments was to find the phase velocity of the slow wave circuit at the operating frequency of the 
device. A good portion of the dispersion curve is displayed to confirm the experiment and to properly characterize the meander line circuit.

\subsubsection{Dispersion Characteristics From Standing Wave Measurements}

An $\mathrm{X}-\mathrm{Y}$ stage was developed to measure the standing wave above the circuit. Fig. 4.6 shows the experimental setup. The circuit is energized with an RF signal at a particular frequency, and the other end of the circuit is terminated into a short. A shorted load yields a very clean and distinct standing wave pattern. To measure the electric field intensity, a small wire sticking out of a coaxial cable was used as an antenna. This antenna closely resembles a simple monopole, but instead of an infinite ground plane, an outer conductor from the coaxial cable is used. Although not perfectly polarized, the 'monopole' antenna is more sensitive to electric fields parallel to it, and in this case the y-polarized electric field. A spectrum analyzer is used to measure the field intensity. Stepper motors are used to step in the $\mathrm{x}$ and $\mathrm{z}$ directions. LabVIEW code was developed to interface with all the equipment, to step the stepper motor, and to take a measurement. An array of electric field intensities can be gathered on the $x-z$ plane right above the circuit to map the standing wave pattern at different frequencies. 


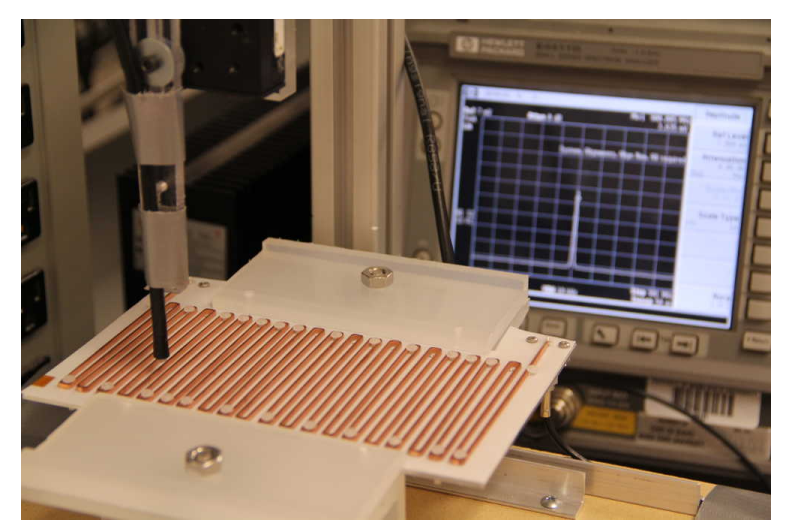

Figure 4.6: Photograph of the standing wave measurement setup. The slow wave circuit sits on top of an $x-y$ stage, and a coaxial cable connected to a spectrum analyzer on one end and the other end is placed right over the slow wave circuit with the center conductor exposed.

To extract the dispersion characteristics from this data, the standing wavelength in the $x$-direction was found using either a 2D spatial FFT or a $1 \mathrm{D}$ spatial FFT along the center of the meander line in the $x$-direction. From transmission line theory, the traveling wavelength is twice the standing wavelength. In this way the wavelength can be determined for each frequency, and the dispersion diagram can be created. More details on this method are given in Section 6.2.2.1.

\subsubsection{S-Parameters}

Using a network analyzer, the S-parameters of the circuit can easily be found. The network analyzer was used to measure the S-parameters up to $3 \mathrm{GHz}$. Because of the periodicity of the circuit, certain cutoff conditions $\left(S_{21}<-20 \mathrm{~dB}\right)$ should exist as outlined in the Chapter 2. The S-parameters are used to confirm the results of the dispersion diagram created by the standing wave experiments. 


\section{CHAPTER 5}

\section{SIMULATION SETUP}

Three different simulation tools were used to study different aspects of the CFA design. COMSOL [41] is used to study the dispersion of the meander line slow wave circuit. SIMION [40] is used to study the electron trajectories, and Vsim [42] is used to study the full electron beam and RF wave interaction. The post processing of the results is generally done in MATLAB [110]. This chapter describes the setup of each simulation tool and of the post-processing techniques to analyze the results.

\subsection{COMSOL Setup}

Simulations were performed in COMSOL to determine the dispersion characteristics of the slow wave circuits via the standing wave pattern and the S-parameters. One approximation was used on the port. Instead of a coaxial port, a simple rectangular port at the end of the microstrip was used. This approach was used to minimize the number of elements in the model. Another approximation is that the Teflon screws of the circuit are absent in the model, also to minimize elements. The domain boundary conditions are perfectly matched layers (PMLs). The general slow wave model is shown in Fig. 5.1 along with the mesh. This mesh quality is the best that can be offered due to the memory limitations of the computer, 15 GB. 


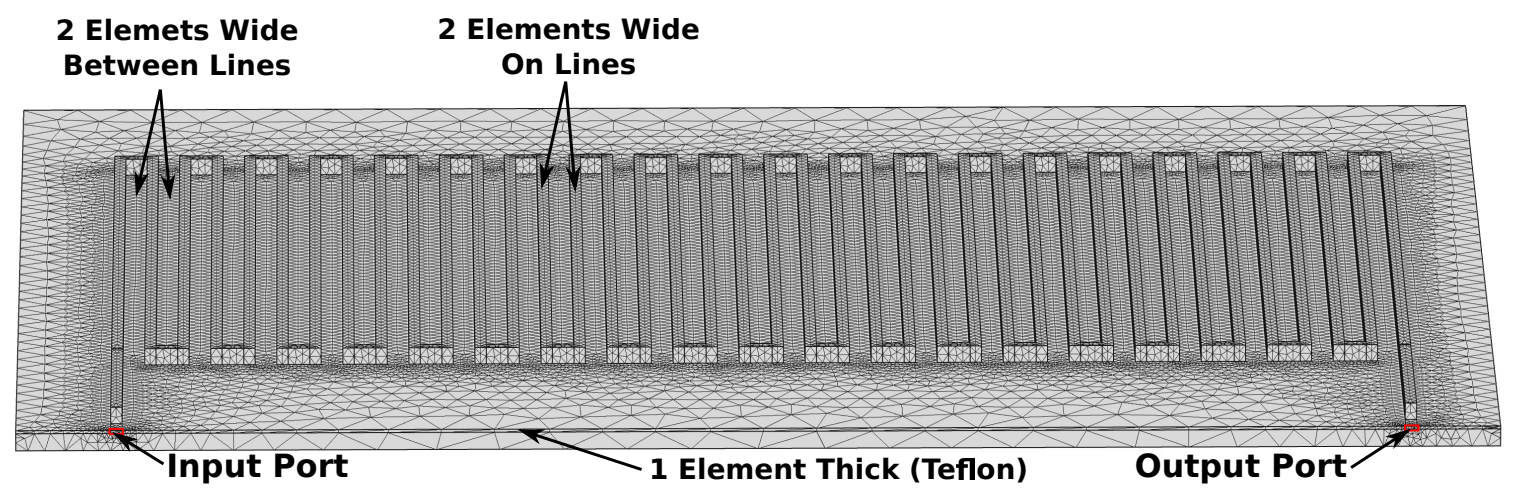

Figure 5.1: COMSOL model for SW2 showing the generated mesh

The regions in the mesh that require a small mesh size are in between adjacent wires, the wire itself, and the Teflon. The best mesh achieved within memory constraints is 4 elements between wires, 4 elements on the wires, and 1 element for the Teflon height for SW1. It was found that the minimal requirements are 2 elements between wires, 2 elements on the wires, and 1 element for the Teflon height. The difference in the results from both cases were very minimal. All simulations presented here are with these minimal requirements for mesh size.

The 'free space' meander line simulations were performed. Also, due to the design constraints of the CFA configuration such as the sole and end hats, these were also added into the simulation to see the effect on the dispersive characteristics. The sole and end hats were implemented by using conducting blocks with no actual structural support. The structural support in the real CFA is far removed from the interaction region and is not needed for the simulation.

\subsection{SIMION Setup}

A side view, normal to the $x$ - $y$-plane, of the 3D CFA setup is shown in Fig. 5.2. The mesh cell size for the electric field calculations is $1 \mathrm{~mm}$. The particle source emits a 
number a particles from a square patch on the cathode. The energy of the emitted particles is of a Gaussian distribution centered about $60 \mathrm{eV}$ with a standard deviation of $5 \mathrm{eV}$ [108]. The direction of the emitted particles is of a cone distribution with a $60^{\circ}$ half angle to model the GFEA emission characteristics.

Each particle corresponds to a possible trajectory. This trajectory is plotted from cathode to the electrode at which it collects. There are 4 electrodes that are monitored: the sole, anode, end collector, and the emitter/gate. By using many trajectories emitted from the emitter with a distribution of energies characteristic of a FEA, the currents at each electrode can be monitored and compared with experiment.

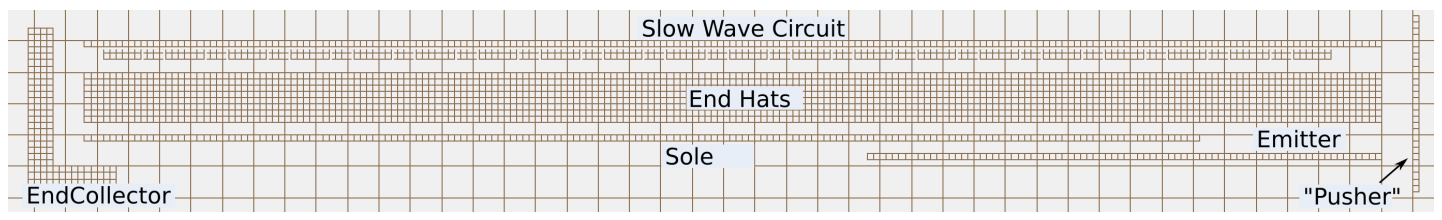

Figure 5.2: SIMION CFA configuration from the side (normal to the $x-y$ plane). Electrons cycloid from right to left in this model.

\section{$5.3 \quad$ Vsim Setup}

The process of setting up the model went through a few stages and different approaches as a part of this research. Some approaches were abandoned and are irrelevant to the working model; however these approaches are valuable for anyone interested in using Vsim and provide valuable insight; therefore descriptions of these approaches are given here.

First, the general model outline is described along with the solvers, geometry, and diagnostics. Then the different grid types are discussed along with their effects on the implementation of the solvers, geometry, and diagnostics. Also, the different types 
of cathode implementations are discussed: the injected beam configuration and two different types of distributed cathode implementations.

\subsubsection{The VSim Model}

The model requires a creation of the geometry on the grid, an electrostatic field solver, an electromagnetic solver, and a particle push algorithm. Fig. 5.3 shows a 3D view of the injected beam configuration on a uniform grid. The electrostatic (ES) solver is needed to implement the cathode, sole, end hats, and slow wave circuit potential. A separate solver is needed to implement the RF waves on the slow wave circuit. The particles need to interact with both the static and RF electric and magnetic fields (via the particle push algorithm). The electrons also generate electromagnetic fields themselves.

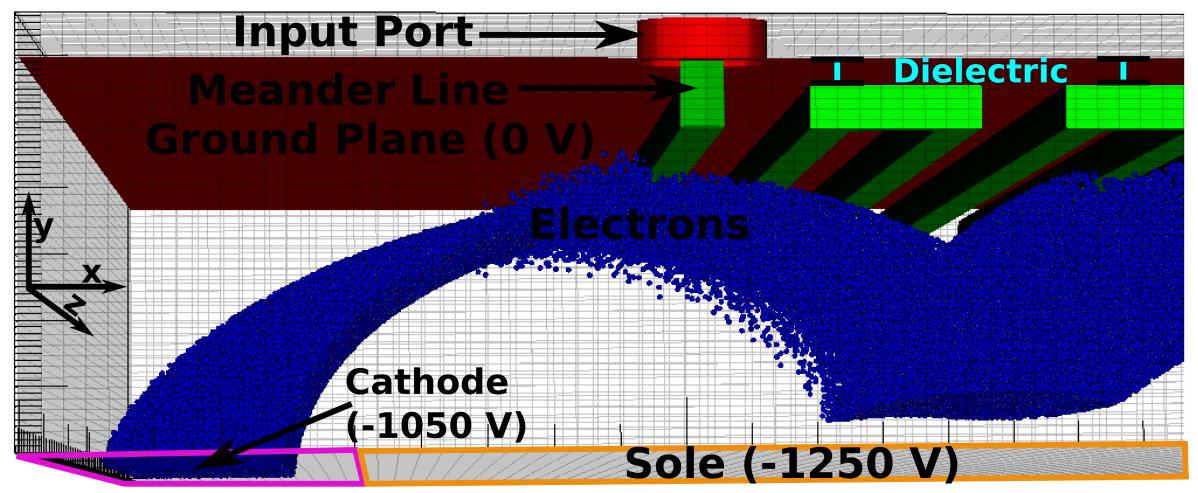

Figure 5.3: Vsim Geometry with electrons. The RF wave is input on the edge of the domain, within the coaxial port. The RF wave travels within the dielectric region between the ground plane and the green meander line. Electrons are emitted from the cathode region, and cycloid right due to the crossed electric and magnetic fields. The electrons interact with the RF wave and give up their energy to amplify the RF wave.

The following is a summary of the model implementation and is explained in detail in the following sections: 
- Define the grid and create the geometry

- Create electrostatic boundary conditions and solve the electrostatic field

- Define RF ports and boundaries and define the electromagnetic solver

- Add both the RF and ES fields to create the total electric field

- Define the particle parameters, sources, and sinks

- Define any diagnostics

Table 5.1 shows a summary of all the parameters involved with the implementation of the model. The following sections describe these parameters in detail. These parameters are referred to for the rest of the dissertation, so this table provides a good reference.

Table 5.1: Summary of Parameters

\begin{tabular}{lll}
\hline Category & Parameter & Description \\
\hline Voltages: & $V_{a s}$ & Voltage from Anode to Sole \\
& $V_{c s}$ & Voltage from Cathode to Sole \\
& $V_{b e}$ & Voltage of the Beam Electrode \\
& $V_{e h}$ & Voltage of the End Hats \\
& $V_{\text {cathode }}$ & Potential of the Cathode \\
& $V_{\text {sole }}$ & Potential of the Sole \\
\hline SW Circuit: & $L_{p}$ & Length of the Pitch \\
& $W_{s w}$ & Width of the Slow Wave Circuit \\
& $L_{s w}$ & Length of the Slow Wave Circuit \\
& $W_{L}$ & Line Width \\
& $H_{L}$ & Line Height \\
& $H_{d}$ & Dielectric Height \\
& $L_{\text {coax }}$ & Coaxial Cable Length \\
& $R_{\text {Icoax }}$ & Radius of the Coaxial Inner Conductor \\
& $R_{\text {Ocoax }}$ & Radius of the Coaxial Outer Conductor \\
\hline CFA Dimensions: & $H_{a s}$ & Height from Anode to the Sole \\
& $H_{c b e}$ & Height from the Cathode to the Beam \\
& $L_{c}$ & Electrode \\
& Cathode Electrode Length
\end{tabular}




\begin{tabular}{|c|c|c|}
\hline Category & Parameter & Description \\
\hline & $L_{e}$ & Emission Region Length \\
\hline & $W_{e}$ & Emission Region Width \\
\hline & $L_{s}$ & Sole Length \\
\hline & $L_{c s}$ & Length between the Cathode and Sole \\
\hline & $L_{c o}$ & Cathode Offset Length \\
\hline & $H_{b e}$ & Height of beam electrode \\
\hline \multirow[t]{3}{*}{ VSim Grid: } & $d X, d Y, d Z$ & Cell length in $\mathrm{X}, \mathrm{Y}$, and $\mathrm{Z}$ \\
\hline & $N_{L W}$ & Number of Cells per Line Width \\
\hline & $N_{d}$ & Number of Cells per Dielectric \\
\hline \multirow[t]{6}{*}{ Other Parameters: } & $I_{\text {beam }}$ & Electron Beam Current \\
\hline & $P_{r f}$ & RF input Power \\
\hline & $P_{g f e a}$ & GFEA Drive Power \\
\hline & $H_{c}$ & $\begin{array}{l}\text { Height of the cathode from } y=0 \text { edge for } \\
\text { divergence free region }\end{array}$ \\
\hline & $L_{e 2 e}$ & $\begin{array}{l}\text { Length between emitter to emitter of } \\
\text { segmented cathode }\end{array}$ \\
\hline & $J_{y}^{*}$ & $\begin{array}{l}\text { Approximated current density used in } \\
\text { cathode approximation } 3\end{array}$ \\
\hline \multirow[t]{7}{*}{$\begin{array}{l}\text { Spatial Emission } \\
\text { Profile: }\end{array}$} & $\phi_{x}$ & $\begin{array}{l}\text { Spatial phase shift used for the sine wave } \\
\text { emission profile }\end{array}$ \\
\hline & $\phi_{t}$ & $\begin{array}{l}\text { Time phase shift used for the sine wave } \\
\text { emission profile }\end{array}$ \\
\hline & $\phi_{\text {offset }}$ & $\begin{array}{l}\text { Phase shift offset from the } R F \text { accelerating } \\
\text { region for the sine wave Profile }\end{array}$ \\
\hline & $J_{p}$ & Peak current density of the emission profile \\
\hline & $I_{t o t}$ & Total emitted current \\
\hline & $f_{D C}$ & $\begin{array}{l}\text { Fraction of the current density that is } \\
\text { uniform }\end{array}$ \\
\hline & $J_{e}$ & Emission current density \\
\hline
\end{tabular}

\subsubsection{Grid}

The grid can be uniform or non-uniform. The cell sizes $(d X, d Y, d Z)$ of the uniform grid remain constant throughout the entire domain; whereas the cell sizes of the 
non-uniform grid can vary in the same direction. The cell size throughout the domain is determined by the resolution requirement of the smallest geometry in the domain. Because of this limitation, the number of cells in a uniform grid can be quite large. The non-uniform implementation can have small cells located where they are needed and increased cells sizes where the resolution requirements are less stringent. A detailed description of the non-uniform and uniform grid can be found in sections 5.3.3 and 5.3 .4 , respectively.

The minimum requirements on the $x$ and $z$ axis are determined by the slow wave circuit line thickness. The geometric dimensions for the top view (normal to the $y$-axis) on a uniform grid is shown in Fig 5.4. Fig. 5.4 does not show the actual dimensions of any slow wave circuits used in this work but is used for a good pictorial representation. Note that in this case, the line width is two cells wide $\left(N_{L W}=2\right)$, which is the same for $d X$ and $d Z$. This resolution is the chosen resolution for the CFA models used in this work. It would be more desirable to increase this resolution, but each increase in number of cells per line width, $N_{L W}$, increases the number of cells in both the $x$ and $z$ axis. Studies on the effects of the $N_{L W}$ show minimal gains to accuracy for $N_{L W}>2$. Also note that due to this coarse resolution, the inner conductor of the coaxial port is square rather than circular. 


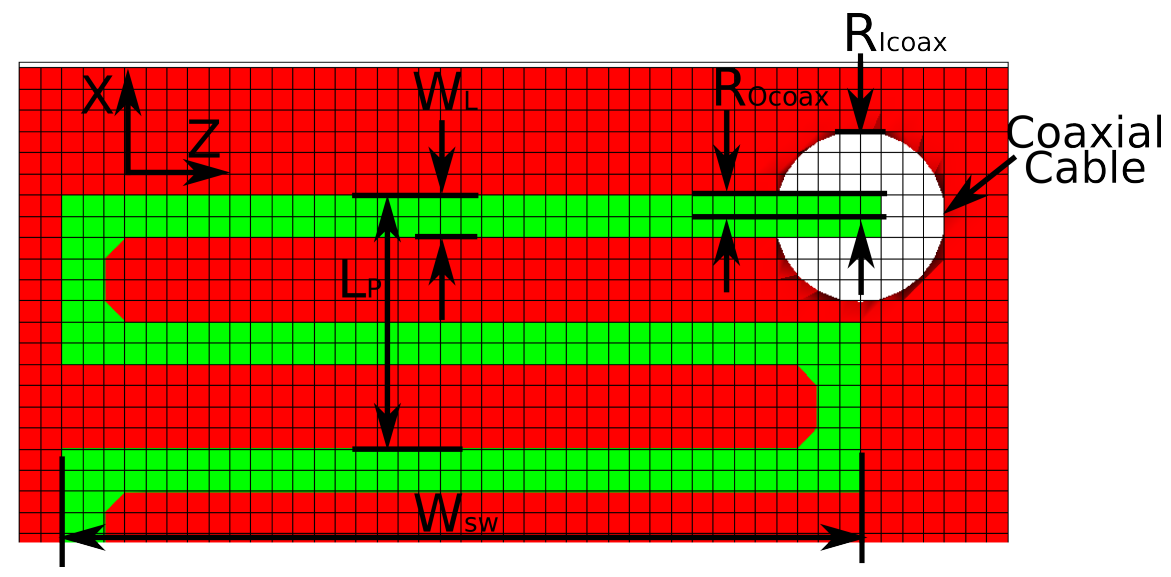

Figure 5.4: View of the dimensions of the slow wave circuit and ports from the top view, normal to the $y$-axis. The green meander line comes down (in the $y$-direction) through the outer conductor of the coaxial cable, and then meanders above the dielectric (not shown) and ground plane shown in red on the $x-z$ plane.

The minimum requirement in $y$ is determined by the dielectric thickness. The geometric dimensions on a uniform grid for the side view (normal to the $z$-axis) is shown in Fig 5.5. These dimensions are the actual dimensions used for SW3, the circuit used in the NU CFA. In this case the number of cells per dielectric, $N_{d}$, is two. These studies are also not presented here for brevity. This resolution is chosen for the simulations used in this work. Once again, more cells would be desirable but would increase the model size greatly. 


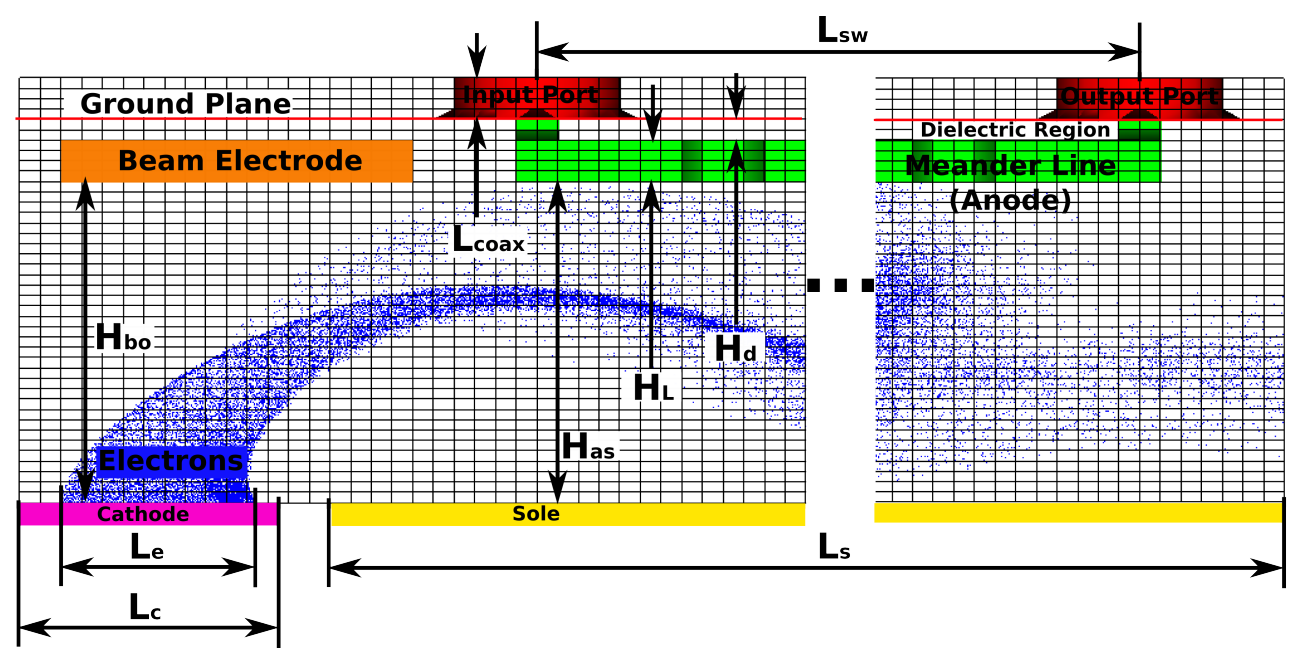

Figure 5.5: View of the VSim model, showing the dimensions of the slow wave circuit and ports from the side view, normal to the Z-axis for the NU CFA study.

\subsubsection{Create the geometry}

There are two methods to create a geometry in VSim, and this section focuses on the first method. The first method uses spatial coordinates to define the geometry rather than cell coordinates. These spatial coordinates are then translated to the grid coordinates for implementation of the finite difference method. This method allows for implementation of complex geometries. The slow wave circuit, the coaxial input and output ports, and all the exterior of the domain are defined using this method. The other method creates boundary conditions using cellular coordinates but is limited to cubic geometries. This method is defined and discussed in the electrostatic and electromagnetic sections. Many of the electrostatic boundary conditions are defined in this way. Also, the dielectric of the slow wave circuit is defined in a similar way.

The geometry is created using a superposition of mathematical functions using spatial coordinates rather than cell coordinates. VSim supplies two main types of 
functions to create the geometry: fill and void. These functions, used in conjunction with mathematical descriptions of the shapes, create the geometry. The fill function fills the defined area with metal, and the void function removes metal from the defined area. Adding many functions like this together, complex geometries can be created. Note that the order in which the fill and void functions are defined affect the end result.

To define different shapes mathematically, a few notable functions are used. The Heaviside function is used to define every shape. By defining mathematical functions which are greater than zero in parts where the desired shape is located, any geometry can be generated. Another important function which speeds up the geometry evaluation process is the modulus function. When creating a periodic geometry, such as the slow wave circuit, each period is defined in the same way but with an offset. One inefficient way to define the periodic structure is to define each segment with its own equation, but with a larger number of periods, evaluating this large set of equations takes time ( $>1$ hour). By using the modulus function, one set of equations which define one period can be used to define the whole periodic structure. This approach reduces the geometry evaluation time to mere seconds.

\subsubsection{Geometric Translation to Grid}

The finite difference method uses a cellular grid to perform all calculations; therefore the spatially defined geometry needs translation to the grid. There are two ways in which the geometry is converted to the grid: using a Dey-Mittra cut-cell approach $[74,75]$ or the stair step approach [73]. Each cell in the stair step approach can only be either conductor or vacuum; whereas the cut-cell approach can "weight" each cell as both conductor and vacuum. The model used in this work is all cubic architecture 
except for the coaxial port. This model does not use the Dey-Mittra cut-cell approach, so the discussion is focused on the stair step.

In order to actually implement the correct geometry, the geometry needs to align with the grid properly. Fig. 5.6 shows (a) the geometry alignment with the grid and (b) the corresponding $y$-component of the electric field of a generic run. In Fig. 5.6(b), the $y$-component of the electric field is defined at the nodes (where the grid lines intersect). Green shaded areas correspond to where the $y$-component of the electric field is zero, which corresponds to conducting regions since parallel electric fields are zero at the boundary of a conductor. Shown in Fig. 5.6(a), the geometry engulfs three nodal points in the width of the circuit. This translates to a conducting region of three nodes, shown in Fig. 5.6 as three nodes of $E_{y}=0$. This corresponds to a line width of two cells which translates to $2 d X$, which is the desired line width.

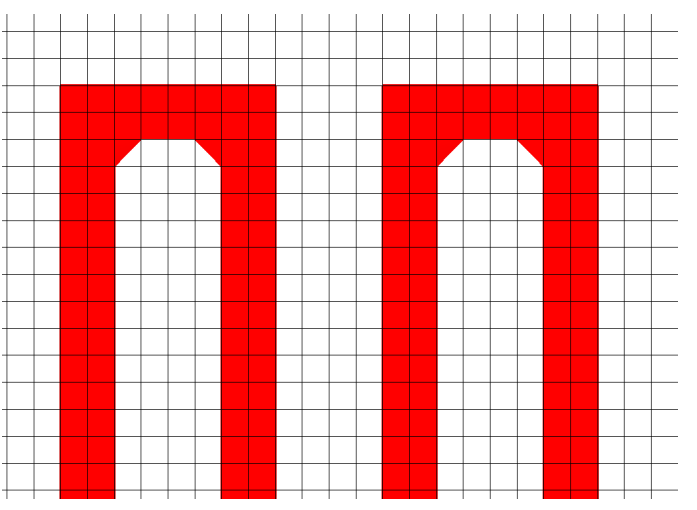

(a)

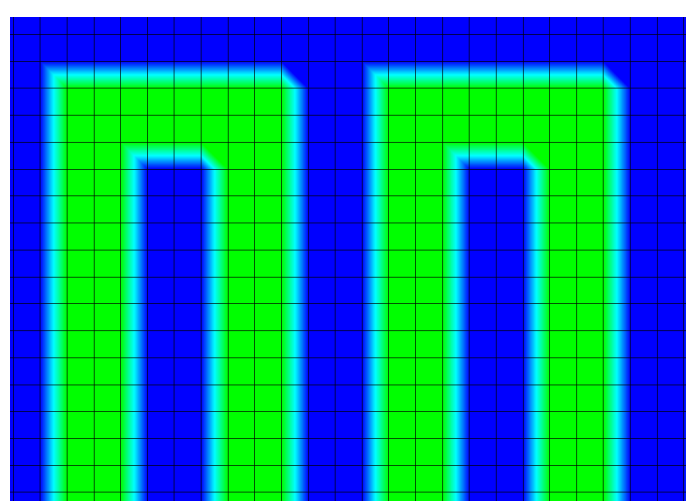

(b)

Figure 5.6: Top down view (normal to $y$-axis) of (a) the meander line geometry with a good alignment with the grid and (b) the corresponding $E_{y}$ field of a generic run . The green section denotes locations where $E_{y}=0$ which corresponds to conductor, and the blue part is vacuum.

Also note that the geometry width is two cells wide plus a small offset in order to ensure that the nodes are actually engulfed. If numerical errors actually make 
the width slightly less than two cells wide, the edge nodes will not be engulfed, and the node will be translated to vacuum erroneously. Fig. 5.7 shows a poor geometry grid alignment and the corresponding Y component of the electric field of a generic run. The geometry appears to be two cells wide but only engulfs two nodes. This translates to only two nodes being conductor which means the actual geometry is one cell wide.

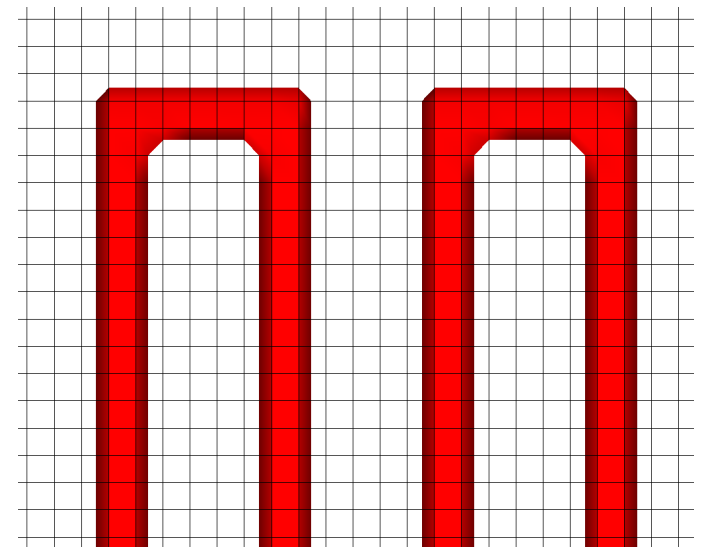

(a)

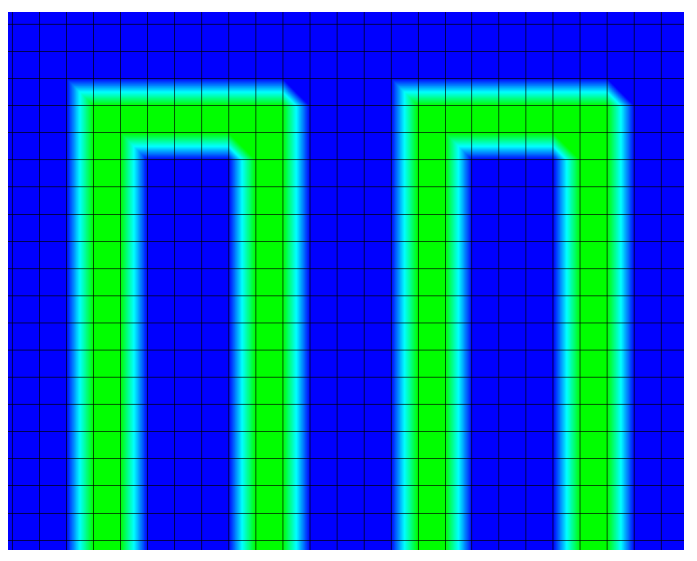

(b)

Figure 5.7: Top down view (normal to $y$-axis) of the meander line geometry with a poor alignment with the grid (a) and the corresponding $E_{y}$ field of a generic run (b). The green section denotes locations where $E_{y}=0$ which corresponds to conductor, and the blue part is vacuum.

The geometry to grid translation also has an effect on the coaxial port. Fig. 5.8 shows the coaxial cable and the corresponding $E_{y}$ field. Denoted by the blue nodes in Fig. 5.8(b), the vacuum portion has two nodes between the inner and outer conductor for most of the region and has only one node on the upper left and lower right corners. It would be desirable to have more nodes in this region to properly resolve the fields, but it is impractical due to computational time constraints. Also, proper modeling of the fields in this region is not that important so long as the power is conserved since 
the main goal of the research is to improve the gain of the device. Also, the length of the coaxial cable from the meander line to the port boundary condition is very small, which minimizes any reflections caused by an impedance mismatch caused by the coarse resolution. Resolution studies were performed on the coaxial ports, and it was confirmed that the power is properly transmitted from the meander line to the coaxial cable. These studies are not presented for brevity.

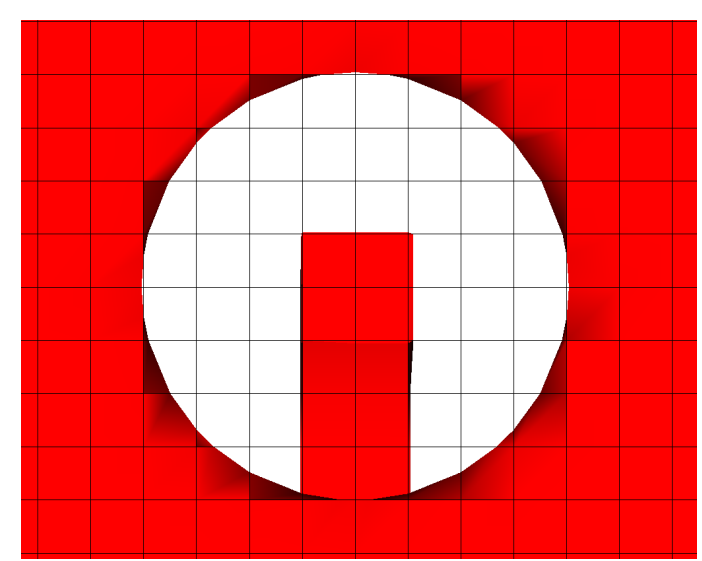

(a)

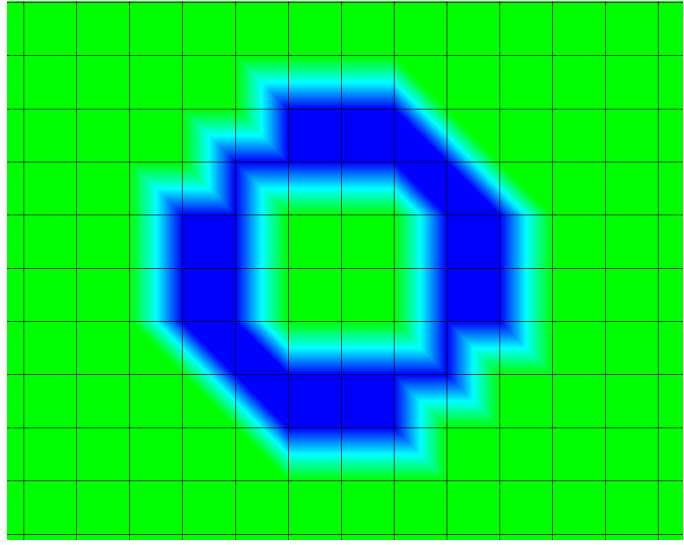

(b)

Figure 5.8: Top down view (normal to $y$-axis) of the input coaxial cable geometry (a) and the corresponding $E_{y}$ field of a generic run (b). The green section denotes locations where $E_{y}=0$ which corresponds to conductor, and the blue part is vacuum.

Proper geometry grid alignment is important, especially with the coarse geometry chosen for the model. If all geometric features are not divisible by a cell length, those features will not be implemented as expected. All geometry in the model is approximated and altered to align well with the grid. These new dimensions change the model from the desired model used in the experiment, but the geometry still provides a close approximation. Table 5.2 summarizes the slow wave circuit (SW3) and CFA parameters used in the NU experiment and the adjusted VSim parameters. 
Table 5.2: Slow wave circuit and CFA dimensions of the NU experiment and the VSim adjustments. Bold listed elements are parameters which are altered in the VSim simulation to align well with the coarse grid.

$\begin{array}{llll}\text { Parameters } & \text { Variable } & \text { NU [cm] } & \text { VSim Model [cm] } \\ \text { Pitch Length } & \mathbf{L}_{\mathbf{p}} & \mathbf{2} & \mathbf{1 . 9} \\ \text { Circuit Width } & W_{s w} & 25 & 25 \\ \text { Circuit Length } & \mathbf{L}_{\mathbf{s w}} & \mathbf{4 0} & \mathbf{3 9} \\ \text { Line width } & W_{L} & 0.3175 & 0.3175 \\ \text { Line Height } & H_{L} & 0.3175 & 0.3175 \\ \text { Dielectric Height } & H_{d} & 0.15875 & 0.15875 \\ \text { Coax length } & L_{\text {coax }} & \mathrm{N} / \mathrm{A} & 0.3175 \\ \text { Inner Coax Radius } & R_{\text {Icoax }} & \mathrm{N} / \mathrm{A} & 0.15875 \\ \text { Outer Coax Radius } & R_{\text {Ocoax }} & \mathrm{N} / \mathrm{A} & 0.635 \\ \text { Anode-to-Sole Distance } & \mathbf{H}_{\mathrm{as}} & \mathbf{2 . 5} & \mathbf{2 . 4 6} \\ \text { Estimated Retardation } & \mathbf{R}_{\text {est }} & \mathbf{3 4 . 5} & \mathbf{3 6 . 2} \\ \text { Emission Region Length } & L_{e} & \mathrm{~N} / \mathrm{A} & 1.5 \\ \text { Emission Region Width } & W_{e} & 10 & 10\end{array}$

\subsubsection{Solve Static Electric Field}

The potential of the boundary conditions (BCs) are defined here. Figure 5.9 shows the electrostatic boundaries of the injected beam configuration from the side, normal to $x-y$ plane. Complex geometries created in the previous section are defined as $0 \mathrm{~V}$. This sets the voltage of the slow wave circuit, the coaxial cable, and the edge of the domain to $0 \mathrm{~V}$. Other boundary conditions, such as the cathode, sole, end hats, and any beam optic electrodes, are defined using the cell coordinates. Periodic boundary 
conditions are defined on the $x$-domain edges. The periodic boundary conditions are used to make the electric field more uniform on the interaction region edges. This minimizes unwanted electric fields near the cathode. Without the periodic BC, the $x=0$ domain edge would be defined as one potential, which would require the beam injection location to be placed further away from the domain edge, increasing the simulation domain size. By applying periodic boundary conditions, the beam injection location can move closer to the edge of the domain without affecting the beam optics, thus decreasing the simulation domain size. This prevents the use of an end collector at the end of the domain, but the end collector has little effect on the electron beam trajectory and is not needed.

After defining the boundary conditions, Poisson's equation is solved using generalized minimal residual (gmres) [69]. The resulting potential fields are shown in Fig. 5.10. The potential is converted to electric fields by calculating the gradient $(\mathbf{E}=-\nabla \Phi)$. This function by default defines the electric fields on the edges. To properly interact with particles, the fields are interpolated to the nodes using the edgeToNodeVec Vsim updater. The electrostatic fields are only calculated once, and the nodal fields are stored so they can be added to the electromagnetic fields later to determine the total electric fields. 

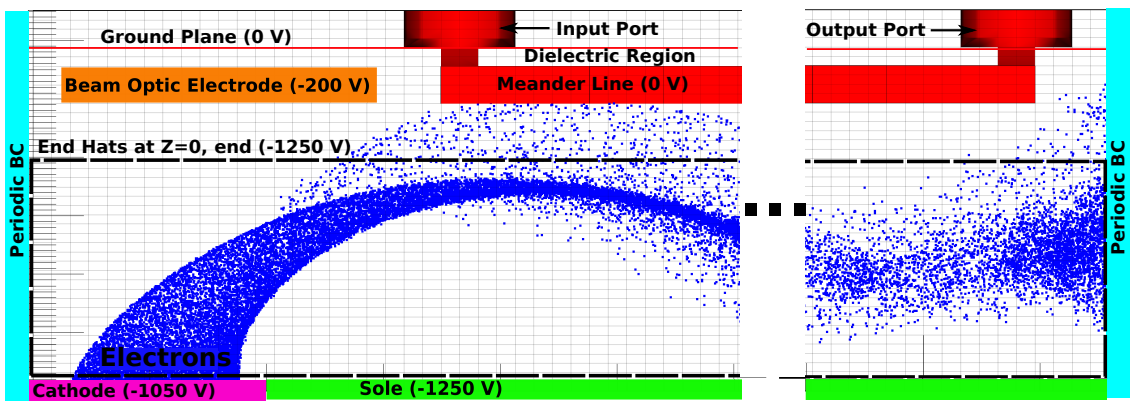

Figure 5.9: The boundary conditions used to control the electron beam injection and cycloid trajectory. The electrons are emitted at a potential $200 \mathrm{~V}$ more positive than the sole so that the cycloiding electrons do not easily collect on the sole. The beam electrode is placed there to control the beam injection into the region between the anode and sole. The end hats are outlined with a dotted line and are at $z=0$ and the upper edge of the $z$ domain. Periodic boundaries are at the edges of the $x$ and $z$ domain. The periodic BCs allow for smaller model by keeping smooth electric fields at the edges.

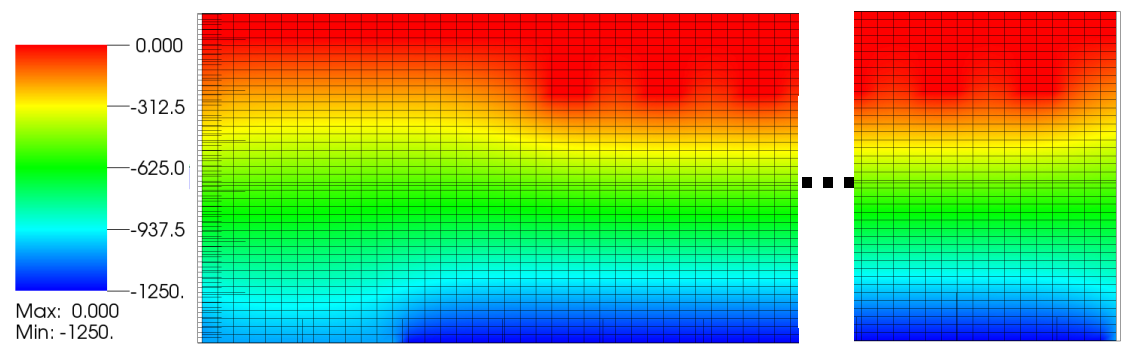

Figure 5.10: Corresponding potentials of the beam optics in the VSim model.

To prevent electron loss along the magnetic field of the device, end hats are used to reflect the electrons back. These electrodes are biased at the same potential as the sole and placed on the sides of the device. Figure 5.11 shows a top down view of the CFA with electron trajectories. Note that with $150 \mathrm{~mA}$, electrons spread out due to space charge and are contained by the end hats. 


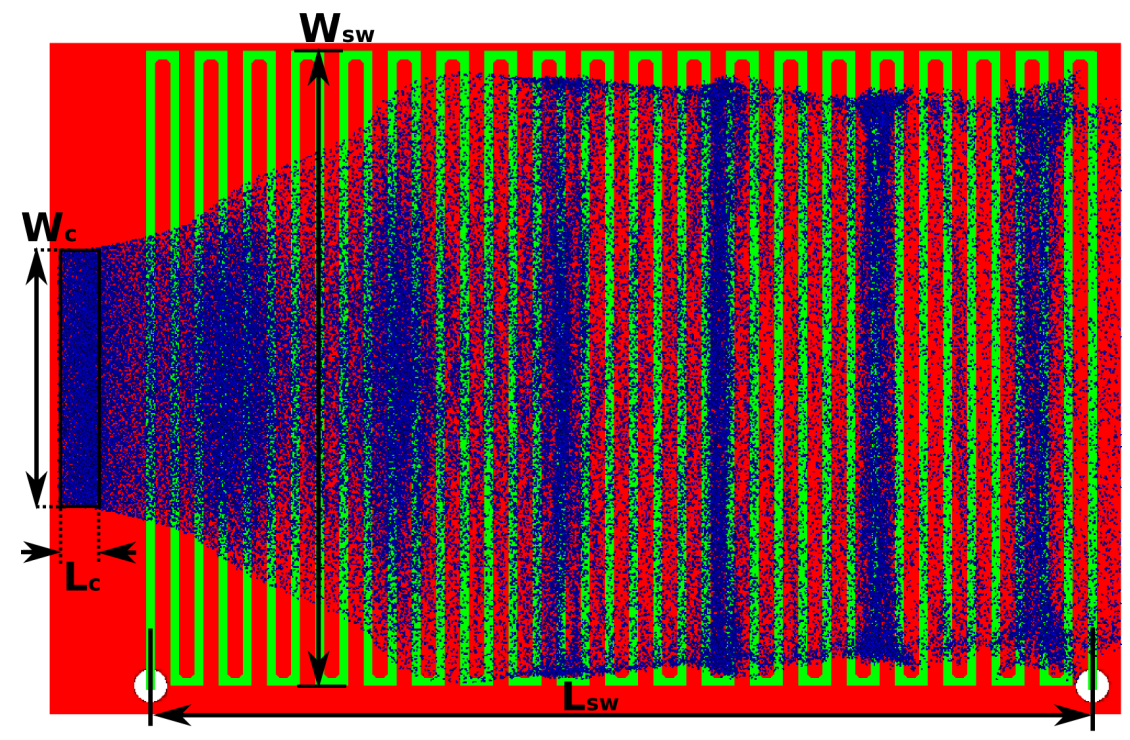

Figure 5.11: View of the dimensions model of the NU CFA with particles from the top view, normal to the $y$-axis. Electrons are shown in blue dots to demonstrate space charge spreading the beam towards the $z$ edges and to show the end hats reflecting the beam back towards the center.

\subsubsection{Solve Electromagnetic Fields}

Vsim has three basic components for solving electric and magnetic fields and stepping through time:

Define fields and BCs: This is where all the needed fields and their boundary conditions are specified. The boundary conditions can be time or spatially dependent. This is where ports, wave launchers, or any field components are defined.

Define updaters: Updaters are the functions to be applied to the fields to step through time. The updaters can be any mathematical function, and they require a field to read from and a field to write to. The updater applies a function to the input fields and updates the output fields. In this way the electromagnetic wave 
can propagate. Updaters can also be used as boundary conditions by setting certain portions of the field to a value. This method is used to implement the input and output ports and the dielectric.

Define the order of operations: This component determines the order in which to apply the updaters.

The simulation domain is defined by the previously created grid boundary. This domain applies conducting boundary conditions $\left(\mathbf{E}_{\|}=0\right)$ on the grid edges. Other boundary conditions can be defined using cell coordinates. These boundary conditions can be used to implement different cathode designs, as discussed later.

Two solvers are used. The Faraday updater reads in the current electric field and updates the magnetic field. The Ampere updater reads in the current magnetic field and any currents and then updates the electric field. To step through time, the solver alternates between the two solvers at each time step. Different boundary conditions, regions, or signals can be input into the solver by directly applying signals or by directly altering the current fields, before the solver is implemented on each time step. This method is used to create the dielectric, input signals on the port, absorb signals without reflection on the port, and send an impulse signal on the circuit to measure dispersion.

The solver and all the boundary conditions are implemented in the following order:

- Input the ES field and store for later use

- Faraday update by a half timestep:

$$
\mathbf{B}(t+0.5 \Delta t)=\mathbf{B}(t)-0.5 \Delta t((\nabla \times \mathbf{E}))
$$

- Any electric signals or boundaries are written to the field here 
- Port updater before the Ampere updater. This is an active port which absorbs waves of a specific phase velocity. This port only is only 1 cell thick rather than the many cells needed for a PML.

- Supply approximated current updaters, if applicable. These updaters are used to approximate the hop funnels used for the distributed cathode which is explained in a later section.

- Dielectric region updater: multiply the electric field located in each cell in the region by $\epsilon_{r}$ which converts $\mathbf{E}$ to $\mathbf{D} / \epsilon_{0}$

- Ampere updater:

$$
\mathbf{E}(t+\Delta t)=\mathbf{E}(t)+\Delta t\left(c^{2}(\nabla \times \mathbf{B})-\frac{1}{\epsilon_{0}} \mathbf{J}\right)
$$

- Dielectric region updater: multiply the electric field located in each cell in the region by $1 / \epsilon_{r}$ which converts back from $\mathbf{D} / \epsilon_{0}$ to $\mathbf{E}$

- Port updater after the Ampere updater

- Add waves to the port

- Faraday update to the full timestep:

$$
\mathbf{B}(t+0.5 \Delta t)=\mathbf{B}(t)-0.5 \Delta t((\nabla \times \mathbf{E}))
$$

- Interpolate the edge fields to the nodes for the particles

\subsubsection{Particles}

Particles are loaded from a 2D cathode area. Each macroparticle is sized so that there are enough particles in the electron beam to provide a smooth distribution. For 
this research, $10^{5}-10^{6}$ particles per macroparticle are used depending on the current. Particles are emitted with an initial electron energy of $5 \mathrm{eV}$. Emitted particles interact with the nodal fields using the Boris-Push algorithm [76]. Particles which enter a Particle sink are removed from the simulation. Fig. 5.12 shows the particle sinks for the injected beam configuration. Particle sinks are created for the sole, the boundary, the end collector, and the cathode. The boundary absorber includes all particles collected anywhere on the boundary but is generally only current which collects on the slow wave circuit. The cathode current helps to indicate current which is emitted but returns to the cathode due to space charge. Note that the dielectric region does not collect currents, and dielectric charging is not implemented.

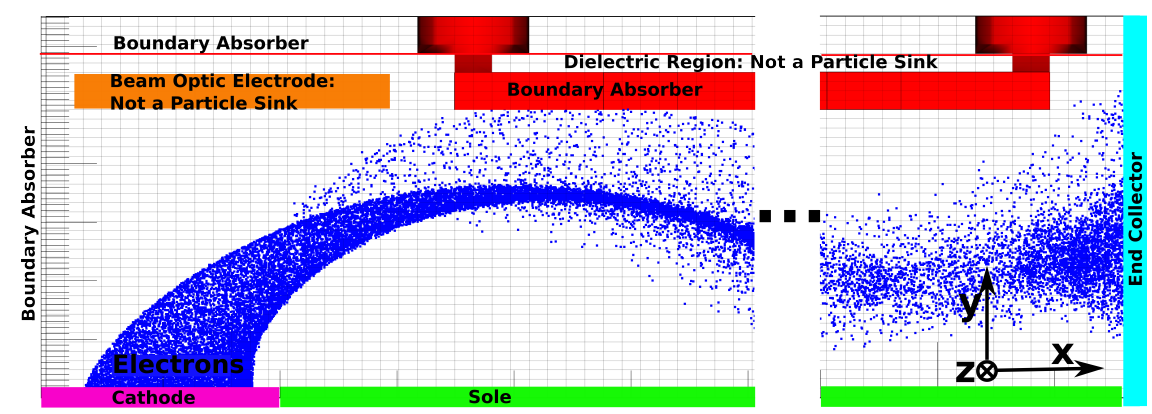

Figure 5.12: Vsim particle boundary conditions showing the the dielectric and beam electrode, which are not particle sinks, and boundary absorber, cathode, sole, and end collector, which are particle sinks.

To update the currents, the particle movement is monitored, and the resulting current density is stored in a deposition field. This field is used during the Ampere updater. The charge density can also be stored in a deposition field but is unneeded because the space charge is self consistent within the electromagnetic solver.

Particle emitters need to have a perfect electric conductor (PEC) boundary condition $(\mathrm{BC})$ in order to short out any fields created by the creation of particles. If charged particles are created within a cell and they leave, they essentially leave an 
opposite charge behind due to the electromagnetic solver. This PEC is there to short out the charge left behind.

Dielectric charging is not implemented in many simulations but can be implemented by collecting particles and not using a PEC BC at the particle sink boundary. Charges enter the cell and are removed for the simulation, but their charge remains due to the electromagnetic solver.

No particle to particle collisions are modeled nor are any particles created from collisions. The only interaction between particles is through the coulomb force between particles through the electromagnetic solver.

\subsubsection{Diagnostics}

There are many diagnostics used in the simulation. A general diagnostic is the standard dump, which dumps all fields at all spatial positions and all macroparticle positions. These are dumped at desired time steps but take up a lot of memory, so they are used sparingly. To view more specific features of the simulation, history diagnostics are used. These can monitor a specific field at specific locations and apply calculations at a desired frequency. The following are a list of the histories used:

Psuedopotential: This diagnostic measures the voltage from the anode to the cathode using the electric field at each time step. It uses the formula: $V=\int_{a}^{b} \mathbf{E} \cdot d \mathbf{l}$. It is used to measure the dispersion of the circuit and is a better choice than just measuring the electric field at one point in the domain because it smooths out much of the noise. This diagnostic is also used to measure the voltage of the input and output ports and at various points on the meander line from the center conductor to the ground plane. This diagnostic can also be used 
in conjunction with the B-Loop diagnostic to determine the impedance of the circuit and to confirm the power.

Number Of Electrons: This diagnostic measures the number of macroparticles. This is to ensure a standard distribution of particles within the domain and compare the spread between simulations.

Emitted Current: This diagnostic is used to monitor the current emitted from an xvLoaderEmitter type of emitter.

Collected Current: This diagnostic measures the current collected on all electrodes. This is an easy way to monitor and visualize where the current is going.

Integrated Poynting Vector: This diagnostic measures the input and output powers. It measures the Poynting vector at each point in the specified area and integrates it.

Field On Line: This diagnostic is used to find the electric fields along a line within the domain. This diagnostic is used to determine the electric amplification as the $\mathrm{RF}$ wave travels down the tube. It is also used to calculate the psuedopotential if the psuedopotential diagnostic is unavailable. It can also be used to replace the B-Loop diagnostic if it is unavailable.

B-Loop: This diagnostic measures the current enclosed in a loop by integrating the magnetic field around the loop, $\mu_{0} I_{\text {enclosed }}=\oint \mathbf{B} \cdot d \mathbf{l}$. This can measure the current on the slow wave circuit. This diagnostic can be used in conjunction with the psuedopotential diagnostic to determine the impedance of the circuit and to confirm the power. 


\subsubsection{Summary}

Each part of the simulation was discussed in detail, and this section describes the connection of these components in the entire simulation. The solving procedure can be broken up into 2 phases: initialize and timestep. The initialize phase includes all the actions which only need to be executed once. The timestep phase includes all the actions which are needed on each timestep. The following list summarizes the process:

- Initial Phase

- Define static electric field boundaries

- Solve static electric fields

- Define electromagnetic boundary conditions

- Define diagnostics

- Time-Step Phase

- Apply boundary conditions

- Solve electric and magnetic fields

- Add static electric field

- Update the particles

- Update and store defined diagnostics

\subsubsection{Non-Uniform Grid Model}

By using a non-uniform grid, the simulation domain size can be significantly decreased, reducing simulation time and memory requirements. The RF solver execution time improves rather linearly with a decrease in the number of cells in the domain. The particle solver execution inherits little to no benefit to a decrease in simulation 
domain size. This is because each particle still requires calculations, and a reduction in the number of cells does not reduce calculations done on each particle. In this model, a reduction of the domain size by half improves RF simulations without particles by about half. This same domain size reduction on simulations with particles reduces simulation times by about $20 \%$.

The non-uniform grid implementation allows for the cell size to change in the same direction. For example, one can change $d X$ as a function of $x$ but not as a function of $y$. The implementation of the grid is performed using 2 vectors: sectionBreaks and deltaAtBreaks. The user defines the cell size at various points, and the program alters the cell sizes in between those points smoothly. The sectionBreaks vector defines the spatial location where the mesh size is defined. The deltaAtBreaks vector defines the cell size at the points defined by the sectionBreaks. For example, with sectionBreaks $=\left[\begin{array}{ll}0.0 & 1.0\end{array}\right]$ and deltaAtBreaks $=\left[\begin{array}{ll}0.01 & 0.1\end{array}\right]$ the cell size at $0.0 \mathrm{~m}$ is $0.01 \mathrm{~m}$ and at $1.0 \mathrm{~m}$ is $0.1 \mathrm{~m}$. The cell size in between $0.0 \mathrm{~m}$ and $1.0 \mathrm{~m}$ is varied smoothly. The cell sizes defined by sectionBreaks is a strict constraint, and if the algorithm cannot vary the cell sizes to match the defined cell sizes, an error is generated. The cell sizes and number of cells in between the defined points is unknown to the user until after running the simulation, which makes any BCs defined by cell coordinates difficult. If the user wants to abruptly change the cell size from one cell to the next, two delta entries are used at the same point where the desired point is. For example, with sectionBreaks $=\left[\begin{array}{llll}0.0 & 0.5 & 0.5 & 1.0\end{array}\right]$ and deltaAtBreaks $=\left[\begin{array}{llll}0.01 & 0.01 & 0.1 & 0.1\end{array}\right]$, the cell size is 0.01 in between 0.0 to 0.5 and 0.1 between 0.5 and 1.0. One should note that geometric features should not lie directly on cell coordinates because inconsistent geometry results. The grid should be slightly shifted off the geometry. This approach does not affect the simulation but 
allows for more consistent results.

Different updaters than the ones used for a uniform grid are required to solve the EM fields. Instead of using Ampere and Faraday updaters, CoordProd updaters are used. These are more generic solvers where the user manually defines the functions. In this case the functions are identical to the ones used in Ampere and Faraday. Dey-Mittra implementation cannot be used with this solver.

The use of a Non-Uniform grid also limits the type of particle emitters available. Only RandDensSrc types of emitters were available at the time of use. Because it is not a xvLoaderEmitter type, the emitted current could not be monitored via the emitted current history diagnostic.

Many of the history diagnostics cannot be used with a non-uniform grid including Psuedopotential and B-Loop. These diagnostics can be created via the use of other histories and some post processing. For example, the psuedopotential can be acquired by using the electric field on line diagnostic and integrating along the line in post processing. This approach requires more hard drive space due to the fact that multiple data points are needed at each time step via the EFieldOnLine diagnostic rather than just one number per time step using psuedopotential.

The implementation in the model uses abrupt changes for the cell sizes. A top down (normal to $y$ ) view of the grid of a generic CFA model is shown in Fig. 5.13. In $x$, the region overlapping the meander line is two cells thick, and the region between adjacent lines is also two cells thick. In $z$, the edges where the meander line runs parallel to $x$, the grid size is $W_{s w} / 2$, and in the middle the grid size is increased to $>3 W_{s w}$. The side view (normal to $\mathrm{Z}$ ) of a generic CFA model is shown in Fig 5.14. The dielectric is 2 cells thick, and this resolution extends into the coaxial cable; the line is 2 cells thick as the sizes are interpolated to match the size in the interaction 
region, and the grid size in the interaction region can be significantly increased. This implementation reduced the number of cells by 4 times. The CoordProdSolver is inherently slower than the uniform solver, but because of the reduction of cells, a net speedup was observed. The RF solver time acquired a $3 \mathrm{x}$ speedup from this domain reduction. The particle solver, however, showed no improvement, and so the total speedup was only about $50 \%$.

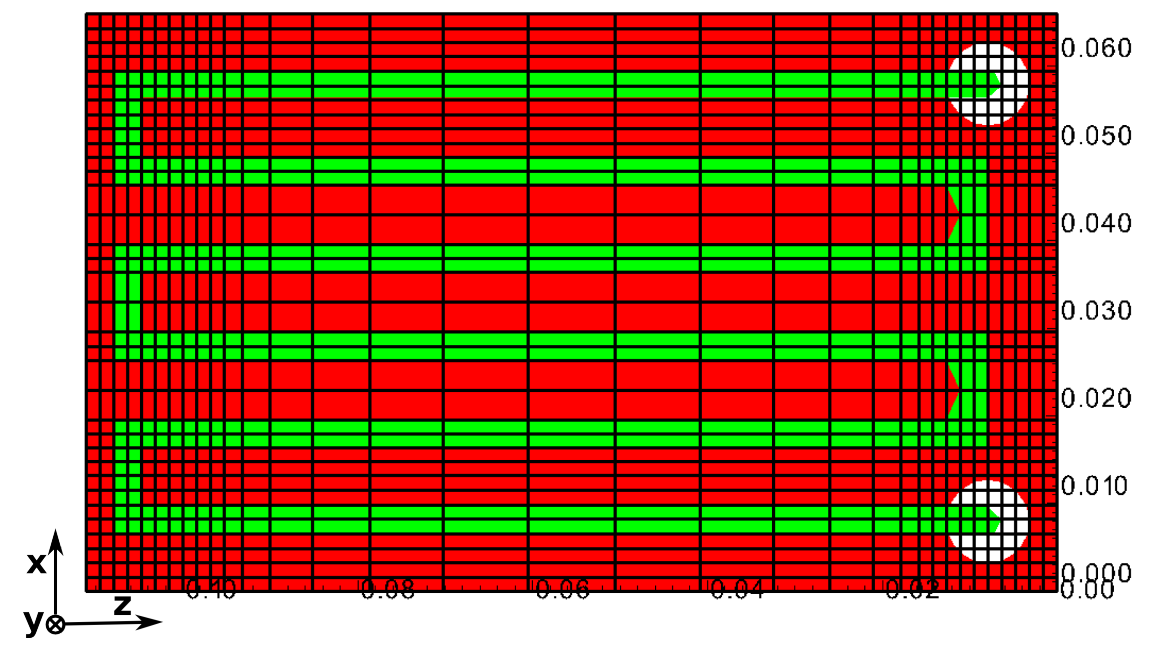

Figure 5.13: Vsim non-uniform mesh on the $\mathrm{X}-\mathrm{Z}$ plane. The green section is the meander line, red is the ground plane, The white circles are the space between the inner and outer conductor of the coaxial cable, and the black lines are the mesh. In $\mathrm{X}$, regions which coincide with the circuit is 2 cells wide and regions between the circuit is 2 cells wide. In $\mathrm{Z}$, the circuit region is 2 cells wide, but in the center, the length of the cells is increased. 


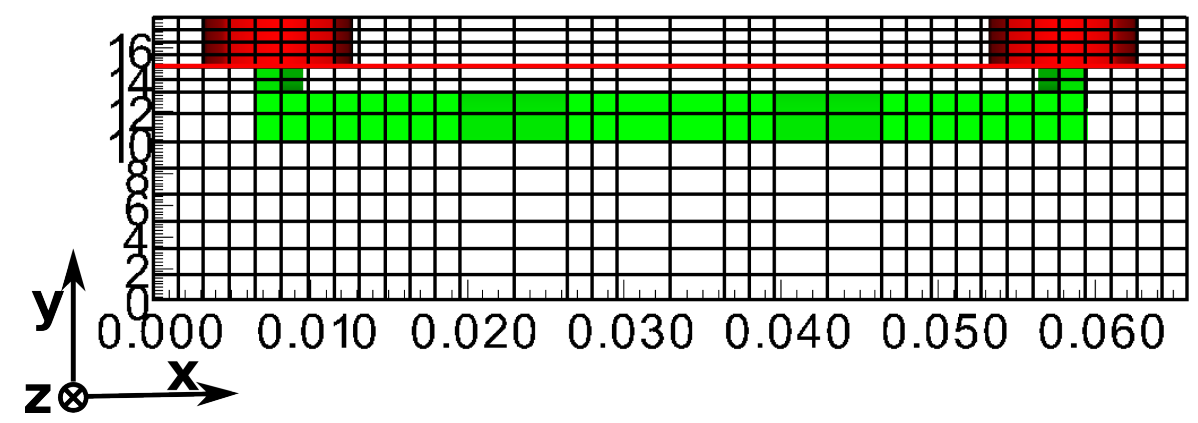

Figure 5.14: Vsim non-uniform mesh on the Y-X plane. The green section is the meander line, red is the ground plane, The white circles are the space between the inner and outer conductor of the coaxial cable, and the black lines are the mesh. In $\mathrm{X}$, regions which coincide with the circuit is 2 cells wide and regions between the circuit is 2 cells wide. In $\mathrm{Z}$, the circuit region is 2 cells wide, but in the center, the length of the cells is increased.

The RF simulations with the non-uniform model showed good agreement with the uniform model. But the simulations with particles showed an anomaly. At the locations of a change in cell size, a charge accumulation was observed. The electric fields at these points acquired a DC bias. The longer the simulation time, the more charge accumulation was observed. Fig 5.15 shows the x-component along $\mathrm{x}$ of an electric field fieldOnLine diagnostic, which shows the charge accumulation. From Gauss's Law, the electric field shows charge accumulation, in the absence of RF fields, by a changing electric field. The CFA simulation was run for 200.0 ns with particles and RF, and then the RF and the electron source were turned off. The electric field in Fig. 5.15 is obtained 200.0 ns after the electron source and RF are turned off. After there were no particles and no RF, except for some tiny stray fields, the electric field should be close to zero. Note that in Fig. 5.15, from $x=0-2.0 \mathrm{~cm}$ the electric field increases smoothly, and for $x>2.0 \mathrm{~cm}$ the electric field varies periodically. For perspective, the periodic variations are larger than the electric field created by the RF 
field alone. The large periodic variations in the electric field are thought to be caused by the cell size changes. This, surprisingly, did not affect the overall performance of the device, and the input and output powers matched fairly well with the uniform implementation. Even with the relatively good agreement in the powers, the huge charge accumulation casts doubt on the results, so the non-uniform implementation was dropped.

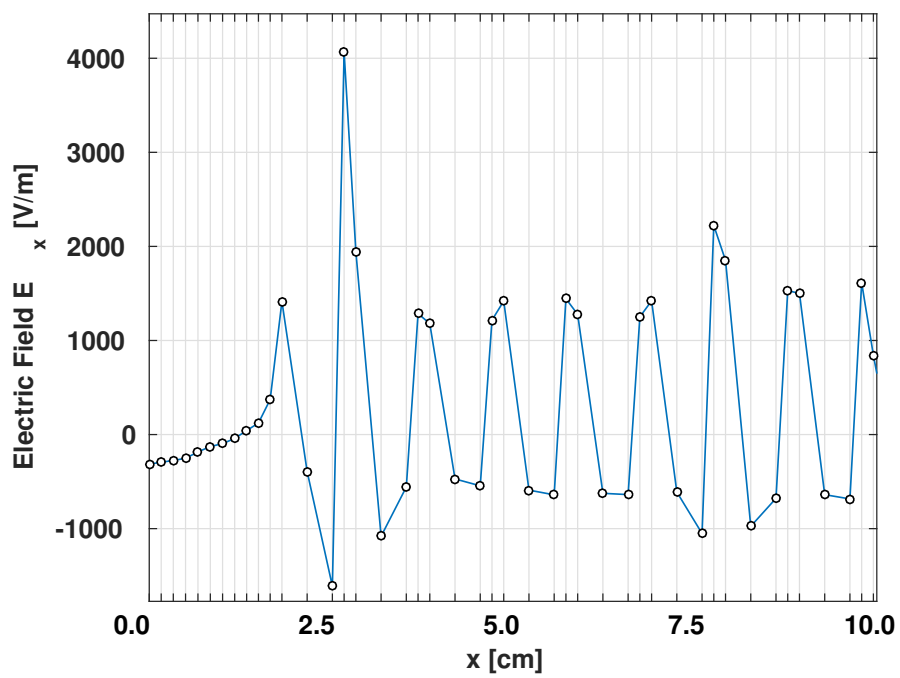

Figure 5.15: E-field diagnostic showing the charge accumulation in the non-uniform grid model.

\subsubsection{Uniform Model}

Because the non-uniform model showed some non-physical results, the working CFA model was changed to a uniform implementation. The uniform model defines the simulation domain size in meters and number of cells. Like the non-uniform model, the line of the SW circuit is 2 cells thick, and the dielectric is 2 cells thick. This defines the grid size everywhere within the domain. All dimensions of the slow wave circuit must be divisible by the cell dimension, so the SW circuit pitch and other dimensions are slightly altered to accommodate this grid. For SW3 the pitch is changed from 
$2.0 \mathrm{~cm}$ to $1.9 \mathrm{~cm}$, and the height of the interaction region is changed from $2.5 \mathrm{~cm}$ to $2.46 \mathrm{~cm}$. This has a small affect on the dispersion characteristics of the circuit. Figs. 5.4 and 5.5, shown in Sec. 5.3.1.1, show the top and side view respectively. Fig. 5.4 does not actually show the actual SW3 dimensions but shows a compact version which easily displays the geometry here.

The uniform model has a few advantages and disadvantages when compared to the non-uniform one. The most important advantage is that the model shows no charge accumulation with particles. The model is easier to conceptualize considering the user only defines the length and the number of cells. All the histories and particle sources can be used. The main disadvantage is that the model takes much

longer to run. The number of cells increased 3-4 times from the non-uniform to the uniform model. RF simulations times, without particles, suffered 3-4 time increase. Simulations with particles suffered a 1.5-2 times increase to simulation time. Also, less resolution is used in the dielectric region in order to keep simulation times practical. Exact representation of the experiment cannot be implemented because the geometric features must be divisible by the cell size.

\subsubsection{Injected Beam Cathode}

The injected beam configuration is shown in Fig. 5.3. Sometimes it is used in conjunction with a beam optic electrode, shown in Fig. 5.9. The cathode electrode is less negative than the sole electrode to allow the cycloidal beam to cycloid down the tube without collecting on the sole. The size of the emission area is comparable to the area of the cathode used in the NU CFA work [14, 15], but the beam optics are very different for the ease of implementation. At lower currents $(<200 \mathrm{~mA})$, the sophisticated beam optics in that work are not needed in the simulation. 


\subsubsection{Distributed Cathode}

The distributed cathode must emit electrons throughout the tube and must prevent cycloidal electrons from collecting back on itself. This requires electrons to be emitted at a potential less negative than what incoming cycloidal electrons see when cycloiding back to the cathode/sole region. This is a contradiction due to the fact that the potential of the cathode/sole region cannot have two simultaneous potentials. The proposed experimental method here was to use hop funnels [36, 37], explained in Sec. 2.6.4. To fully simulate the hop funnels would be computationally expensive. The resolution required to simulate the hop funnels would make the simulation unfeasible. The hop funnels are on the order of $1 \mathrm{~mm}$ and require 10-100 cells to resolve, and to simulate them in conjunction with the CFA which is tens of centimeters long, would take too much time.

To implement this distributed cathode in simulation and keep the grid resolution requirements relaxed, three methods were proposed. The first and third methods are similar to each other and are physically impossible, but have the virtue of being an easy and versatile implementation in simulation. The second implementation is physically possible, but represents a very coarse implementation of something like

hop funnels. The third implementation is used for the distributed cathode work in this dissertation.

\section{Cathode Approximation One: Raised Cathode With Virtual Conductor}

This approximation emits electrons from a location a few cells above the sole from a location within the vacuum. This implementation is not used for the final simulations but may be insightful about the operation of the CFA. This cathode approximation is 
shown in Fig. 5.16. The approximation is identical to the injected beam model except the cathode potential is removed, and electrons are emitted above the sole within the interaction region. When emitting electrons within the domain, the electromagnetic fields must be shorted out in order to prevent a charge build up at that cell. A region called $E_{R F}=0$ region is introduced here. This region applies a conducting boundary condition $\left(E_{\|}=0\right)$ right at the boundary and a $E=0$ within the region. Particles, however, are allowed to enter and leave the region. Also, the static electric field remains untouched in this region. Particles can enter the region, be affected by the static electric field, and leave. This implementation allows particles to be emitted at a higher potential and cycloid down the tube without being collected but at the cost of shorting out the electromagnetic fields immediately adjacent to the sole. This, of course, is physically unrealistic, but since the $E_{R F}=0$ region is far away from the circuit, it was thought that the effect on gain would be minimal. It was believed that because the majority of the bunching and energy coupling between the electron beam and SW circuit occurred near the circuit, the $E_{R F}=0$ region would not affect gain. However, this assumption was shown to be incorrect. A study of the sensitivity of the region is provided in sec. 7.5.1. 


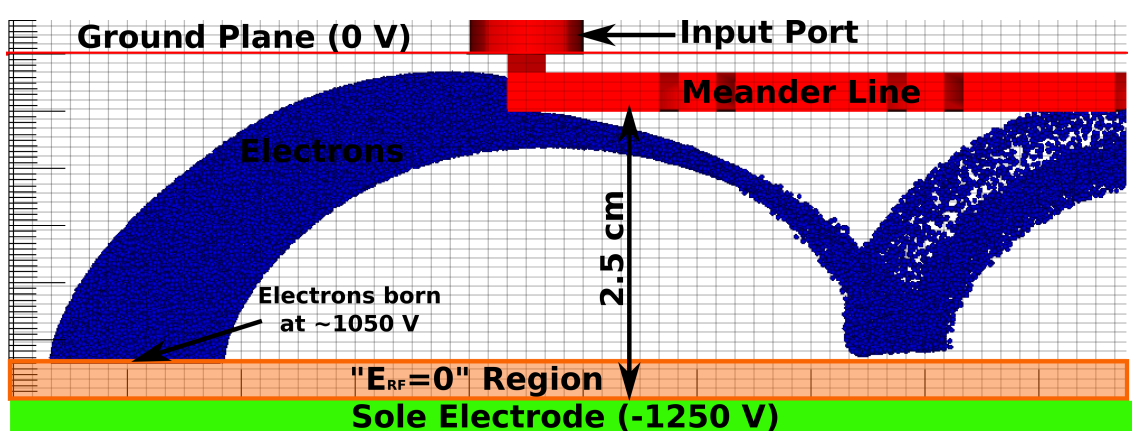

Figure 5.16: Cathode/Sole approximation one. The $E_{R F}=0$ region is placed right above the sole electrode so that electrons can be emitted into the interaction region from a potential less negative than the sole. The $E_{R F}=0$ sets the electric fields equal to zero to prevent accumulation of charge at the electron emission location.

\section{Cathode Approximation Two: Segmented Cathode}

This approximation coarsely resolves the cathode and the sole. The idea here is to have small cathode sections surrounded by longer sole sections. Electrons are emitted from the cathode sections and cycloid down the tube, but when they cycloid back towards the cathode/sole region, they are more likely to see the sole potential and are repelled. The logical method described here, where electrons are emitted directly from the cathode region, has undesirable trajectories because the fringe electric fields caused by the cathode and sole regions are so close to each other. It was found that the electron emission points should be offset slightly in order get the desired results. Both implementations are discussed here.

The first implementation of this approximation is shown in Fig. 5.17. A close up of the geometry is shown in Fig. 5.18 which shows the dimensions. The pink regions are at the cathode potential, and the green regions are at the sole potential. The cathode and sole are separated by a cell so that the fringe electric fields near the cathode edges are weaker. Also, the cathode and the sole are offset from the $Y=0$ domain edge 
so that the cathode and sole can be separated by vacuum. Having the cathode/sole region on the domain edge requires the separating region to be defined, which would cause undesired fringe fields. This cathode/sole offset requires a $E_{R F}=0$ region to be defined in order to short out any charge accumulation at the cathode injection points. Also, the $E_{R F}=0$ region must collect particles; because if it does not, particles end up hovering at the boundary indefinitely because of the fringe electric fields.

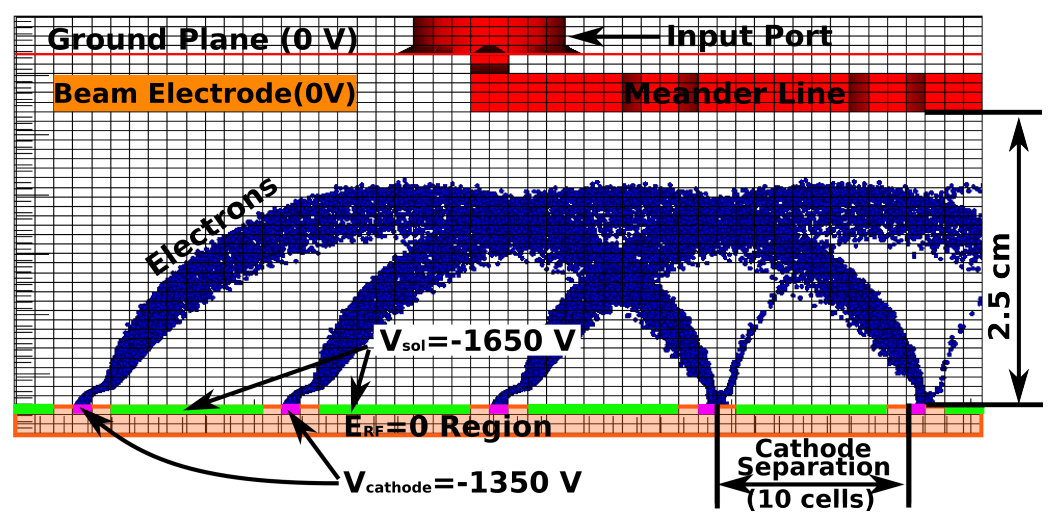

Figure 5.17: The first configuration of the cathode/sole approximation 2 . Cathode potentials are pink, and sole potentials are green. Only the first three cathode potentials emit electrons. Electrons in this case have an 'erratic' trajectory as they leave the cathode. Also, the cycloid radius is a multiple of the cathode separation length, and many electrons just squeeze right back through the cathode potential at the right two cathode potential locations and are collected on the cathode/sole region. 


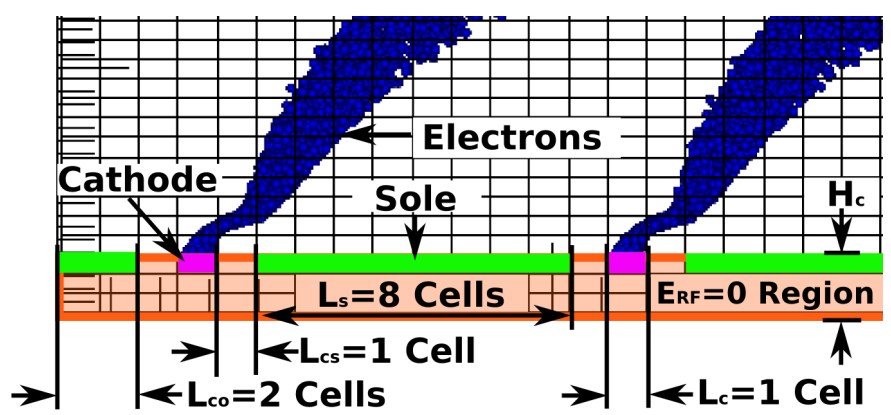

Figure 5.18: A close up on the cathode/region of cathode approximation two, showing the dimensions. This view also shows the 'erratic' electron trajectories as they leave the cathode

Note that the electron trajectories in this implementation can be 'erratic.' In Fig. 5.18 the current comes off the cathode in a curved trajectory. Depending on $V_{c s}$, $V_{a s}$, and $B$, other awkward trajectories can be observed, and this configuration is susceptible to losing current to the cathode/sole region right at the injection point. These erratic trajectories are caused by the fringe fields. The trajectories in Fig. 5.18 are not that erratic, but the gain was difficult to optimize with this type of cathode. In Sec. 7.5.2 a detailed description on the gain optimization is discussed. Note that with the cathode separation of 11 cells at this electric and magnetic field, the cycloid radius is aligned with a cathode potential segment farther down the tube, so most of the current is allowed to collect back on the cathode/sole region. To decrease this lost current, the cathode separation can be changed. This type of injection, however, never yielded the optimum gain, and the erratic trajectories were always present. For this reason, a new cathode configuration was used.

To improve the emission, the electron sources were moved to the right, in the buffer region between the cathode and sole. Fig. 5.19 shows the new electron emission placement. The $x$-component of the electric field in this region points to the right, which causes a force to the left on the electrons. This fringe force 
in combination with the magnetic field, which applies a force to the right as the electrons are leaving the cathode, causes the electron stream to move up away from the cathode into the interaction region in one 'clean' stream. The potential at which the electrons leave is not exactly known because they are born in between the cathode and sole potentials, somewhere between $V_{\text {cathode }}$ and $V_{\text {sole }}$. Also, this implementation is physically impossible because electrons are emitted from within a vacuum region, but the approach allows simulation of the distributed cathode using a coarse grid.

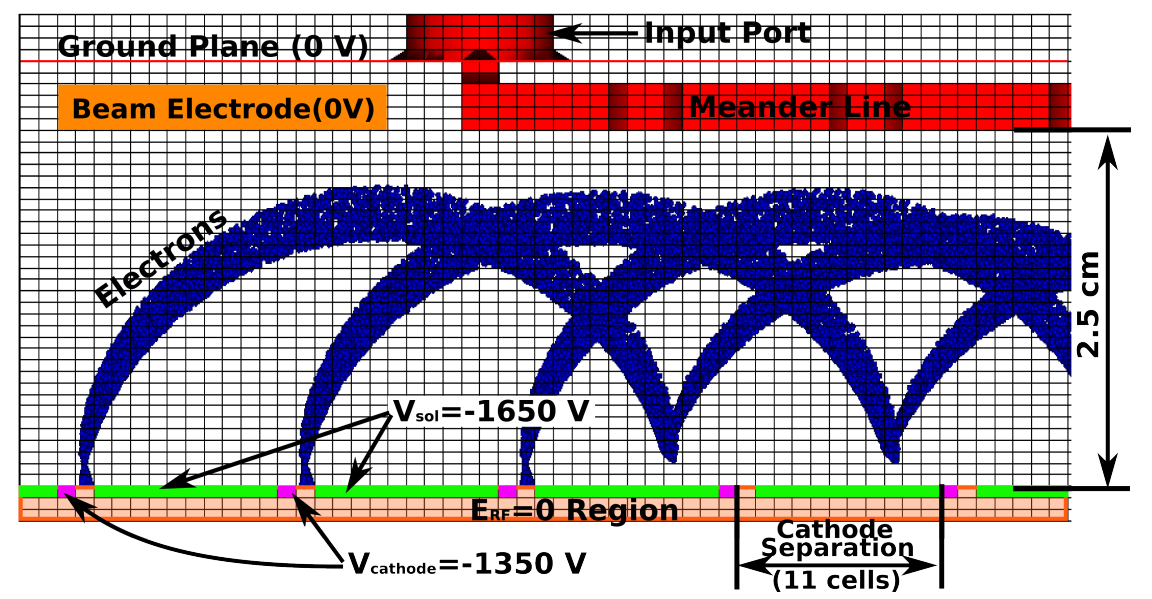

Figure 5.19: The second configuration of the Cathode/Sole approximation 2. Electron emission points are offset to the right of the cathode potentials (pink) in between the cathode and the sole. The cathode separation is optimized so that cycloid radius is offset from cathode segments down the tube and electrons can be repelled back into the interaction region.

This new configuration showed better gain than the previous configuration. Note that the cathode separation in Fig. 5.19 is optimized so that the cycloiding electrons approach the cathode/sole region on the sole part, and all the current is repelled back into the interaction region. The parameters shown in the figure are the optimized parameters for the highest gain. Details on the optimization are given in Sec. 7.5.2.

This approximation requires the cathode regions to be small, and the sole regions 
to be larger. This way the cycloidal electrons are more likely to 'see' the sole potential and be repelled. Also, the distance between cathodes needs to be optimized so that it does not coincide with the cycloid radius. With these small cathode regions, space charge can be an issue. Also, the fringe fields can cause unwanted beam trajectories. Care must be taken to properly inject the desired current. Another effect is that the cathode/sole approximation affects the electric field within the interaction domain, which can affect the $\mathbf{E} \times \mathbf{B}$ velocity.

\section{Cathode Approximation Three: Raised Cathode With Approximated Cur- rent}

This approximation is very similar to the first approximation, but instead of a $E_{R F}=$ 0 region, a region which supplies an approximated current is used to prevent charge from accumulating at the point of injection. To emit electrons at a potential less negative than the sole, electrons are emitted above the sole in vacuum, within the interaction region. To prevent an accumulation of charge at the point of injection without greatly affecting the electric fields and electron movement in this region, a divergence free region is used.

The divergence free region attempts to make the divergence of current density equal to zero everywhere, $\nabla \cdot \mathbf{J}=0$. To make the divergence equal to zero everywhere, an approximated linear current density in the $y$-direction, $J_{y}^{*}$, is supplied to each cell with $\nabla \cdot \mathbf{J} \neq 0$ so that $\nabla \cdot \mathbf{J}+J_{y}^{*}=0$. On each timestep, the approximated linear current density is applied to cells with $\nabla \cdot \mathbf{J} \neq 0$ which will most likely cause the cell below to have $\nabla \cdot \mathbf{J} \neq 0$. On the next timestep, the cell below will also apply an approximated linear current density. In this way, the current will propagate all the way to the conductor on the $y=0$ edge. By having the current originate from a 
conductor, the infinitely growing electric field is prevented. Fig. 5.20 shows the steps for the approximated current to propagate to the edge of the domain. 

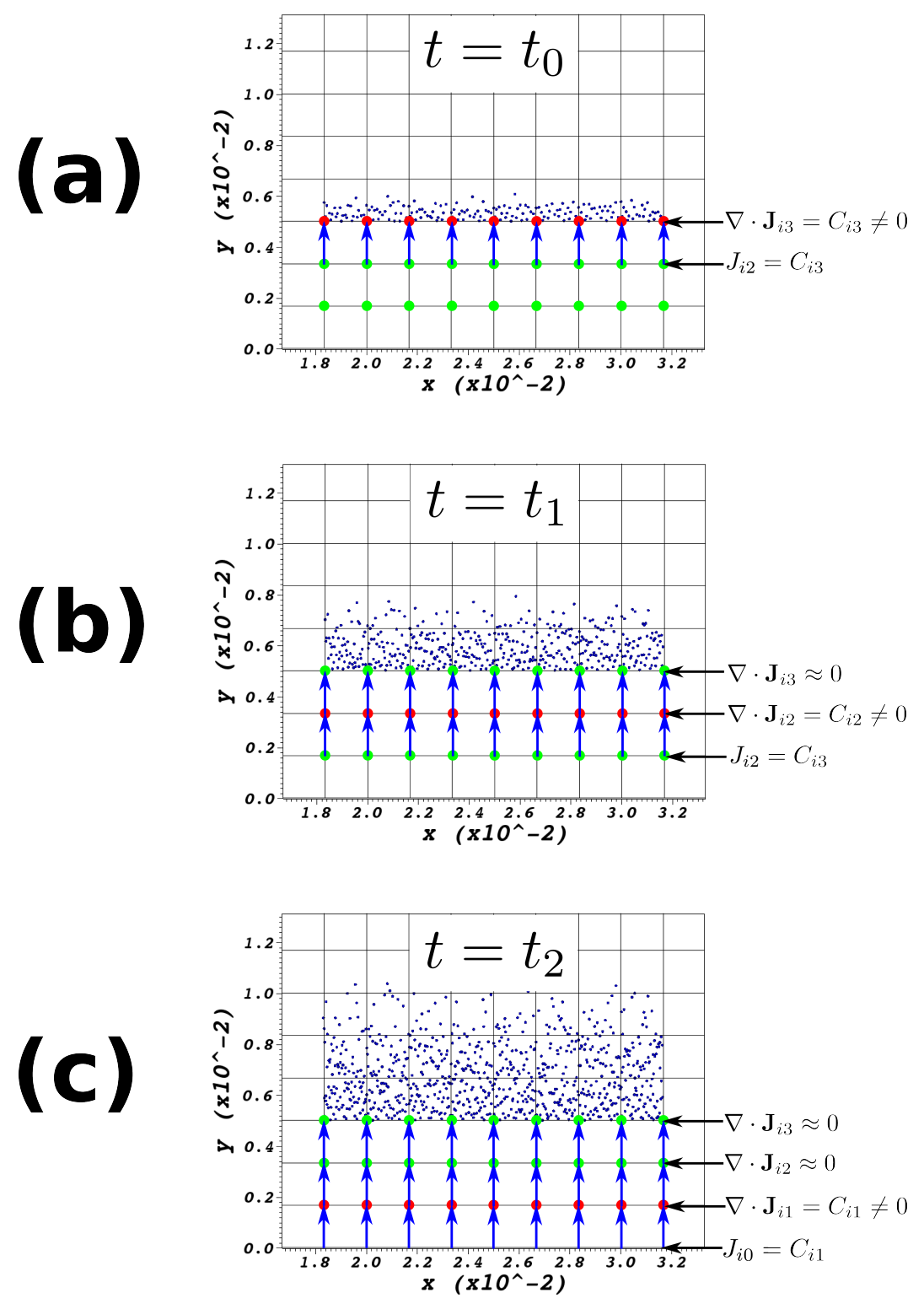

Figure 5.20: A simple example of the divergence free region current propagation from the injection point to the domain edge at (a) $t=t_{0}$, (b) $t=t_{1}$, (c) $t=t_{2}$. Red dots indicate cells where the divergence is not equal to zero, and green dots are divergence free points. Index notation is used where $\mathbf{J}_{i j}$ indicates the current density at cell number $i$ in the $x$-direction. The divergence free region in this example is 3 cells high, and takes 3 timesteps for the current originating from the third row of cells to propagate to the $y=0$ edge. 
The approximated current propagation shown in Fig. 5.20 takes 3 timesteps to reach the $y=0$ domain edge. This is because the electron emission point is 3 cells above the edge of the domain in this example. On the first timestep, shown in Fig. 5.20 (a), current is emitted on the top of the third cell, and because current is being created here, the divergence is not zero $\left(\nabla \cdot \mathbf{J}_{i 3}=C_{i 3}\right)$. Note the use of index notation where $\mathbf{J}_{i j}$ indicates the current density at cell number $i$ in the $x$-direction, and cell number $j$ in the $y$-direction. Red points in the figure are points where the divergence is not equal to zero, and green points are points where the divergence is zero. In order to make the divergence equal to zero on each cell on the third row of cells, the cells below add an approximate current equal to the divergence of the cells above. This causes the divergence on the second row to not equal zero, but on the next timestep shown in Fig. 5.20(b) an approximated current is applied to the first row of cells. This causes the first row to have a non-zero divergence, and on the text timestep, shown in Fig. 5.20(b), applies an approximated current on the boundary. This approach allows the electron current to originate from the conductor while being born from a different potential. In this case, since the current is emitted 3 cells above the boundary, it takes 3 timesteps to propagate the approximated current to the domain edge. Note that the approximated current constantly applies a current density to the cell directly below cells where the divergence is not zero. In this way, small numerical errors or fluctuations in current are accounted for exactly.

This iterative method was found to be the best method to prevent any accumulation of charge from the current emitted within the domain. Because the emitted current density is known to the user, another method is to manually supply the current on each cell from the $y=0$ edge all the way to the emission site. This method helped, but slight errors still caused significant charging. The only way to prevent charging 
required reading the divergence everywhere and setting it to zero iteratively.

\section{Divergence Free Region Side Effects}

A complete study of the divergence-free region effects in the full simulation is provided in Sec. 7.5.3. The approximated current density is found to have a small effect, but there are specific limitations and specific phenomena associated with this method. This section outlines the limitation and effects caused by the approximation.

As a consequence of requiring all divergence equal to zero in this region, any desired electric fields created by current, such as cycloiding current entering the region, will also be set to zero. Ideally, only the emitted current will be accounted for, and incoming current ignored. There is no easy way to differentiate between emitted and cycloiding current without error. This causes the divergence free region to introduce unwanted fields, but the effect on the simulation was acceptably small. Fig. 5.21 shows the cycloiding electron's effect on the divergence free region on the electric field compared to a simulation without the region. 


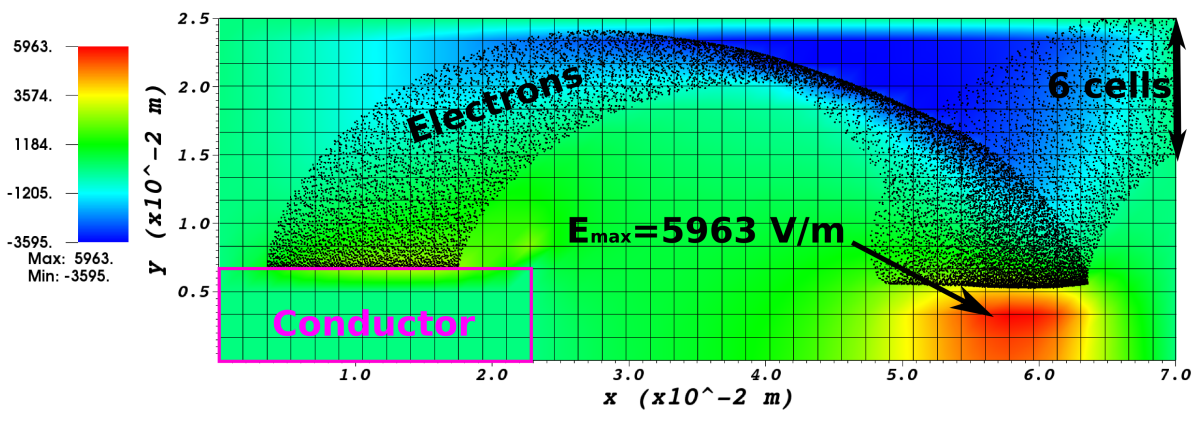

(a)

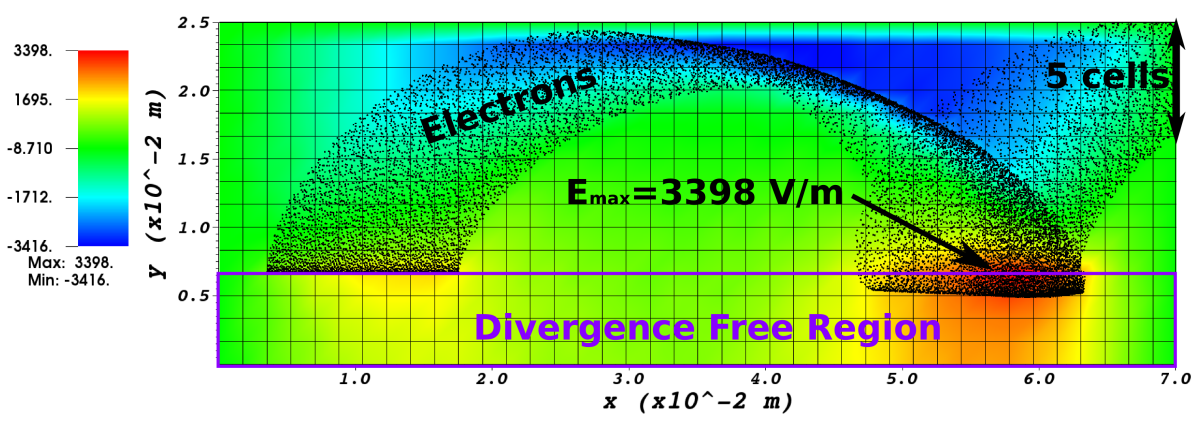

(b)

Figure 5.21: The $y$-component of the electric field overlaid with cycloiding electrons in a (a) standard vacuum region and in a (b) divergence free region. The electron beam spread at $x=7.0 \mathrm{~cm}$ is shown in the figures to emphasize the difference in electron beam trajectories.

There are two major effects on the simulation from electrons cycloiding into the divergence free region: the electric fields between the electrons and the sole at $y=0$ appear smaller, and there is less space charge effect on the electron trajectories. The electric field between the electron beam and the sole without a divergence free region, shown in Fig. 5.21(a), is stronger than the electric field with the region, shown Fig. 5.21(b). Consequently, the larger electric field in Fig. 5.21(a) spreads the electrons out more, 6 cells shown at $x=7.0 \mathrm{~cm}$, after the cycloid rather than the spread in Fig. $5.21(\mathrm{~b}), 5$ cells shown at $x=7.0 \mathrm{~cm}$. The electron beam near the sole creates 
a static electric field field between the beam and the conductor. The reason the electric field between the beam and the sole, when using the divergence free region, is smaller is due to the charge within the beam being farther from the sole. The actual beam approaches the sole within about 3 cells. With the divergence-free region, the electron beam also approaches within 3 cells, but because charge does not exist in the divergence free region, the electric field occurs from the top of the divergence free region to the sole, about 4 cells. This apparent larger distance decreases the electric field there, and consequently, space charge effects at the bottom of the cycloid are weaker.

Another effect of emitting electrons from a divergence free region rather than from a conducting $\mathrm{BC}$ is the electric field caused by space charge at the emission site is not so strong. Fig. 5.22 shows the y-component of the electric field overlaid with electrons of two different cathode implementations. Fig 5.22(a) shows an example of current emitted from a conductor, and Fig. 5.22(b) shows current emitted from a divergence free region. Note that the electric field is stronger in Fig. 5.22(a) than 5.22(b). This difference in Fig 5.22(a) is because the charge in the electron beam is immediately adjacent to the emitting conductor, so the electric field is stronger. In Fig. 5.22(b) the charge in the beam is 4 cells away from the conductor, so the field is weaker. Note that even though the divergence free region supplies current from the $y=0$ edge, no charge exists in the region. So the electric field shown in Fig. 5.22(b) is just the static electric field from the charge in the beam to the edge. This weaker electric field at the emission site reduces the space charge limited current effect, which allows a larger current to be injected than normally would be allowed. 


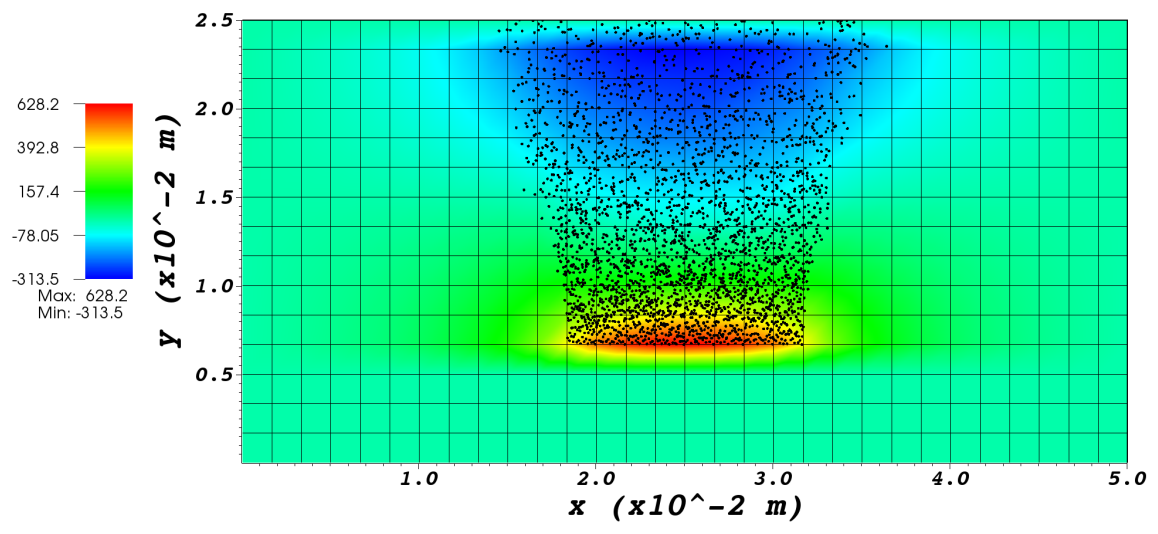

(a)

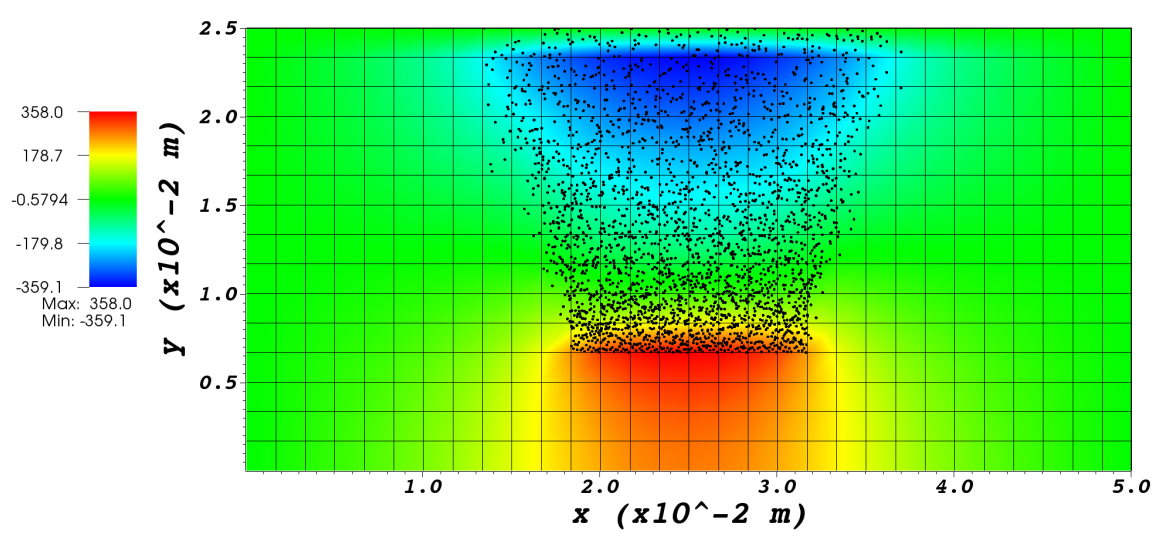

(b)

Figure 5.22: The y-component of the electric field overlaid with electrons electrons in (a) a standard vacuum region and (b) in a divergence free region

Even though the space charge effects at the emission site are smaller when using the divergence free region, electrons look to spread out more. This is due to the $x$-component of the electric field at the emission site. Fig. 5.23 shows the $x$-component of current emitted from a conductor, 5.23(a), and current emitted from a divergence free region, 5.23(b). The conductor in Fig. 5.23(a) shorts out the $x$-component of the electric field created by the beam; whereas the divergence free region in Fig. 5.23(b) 
does not. This added $E_{x}$ field right at the emission site spreads the beam out a little more.

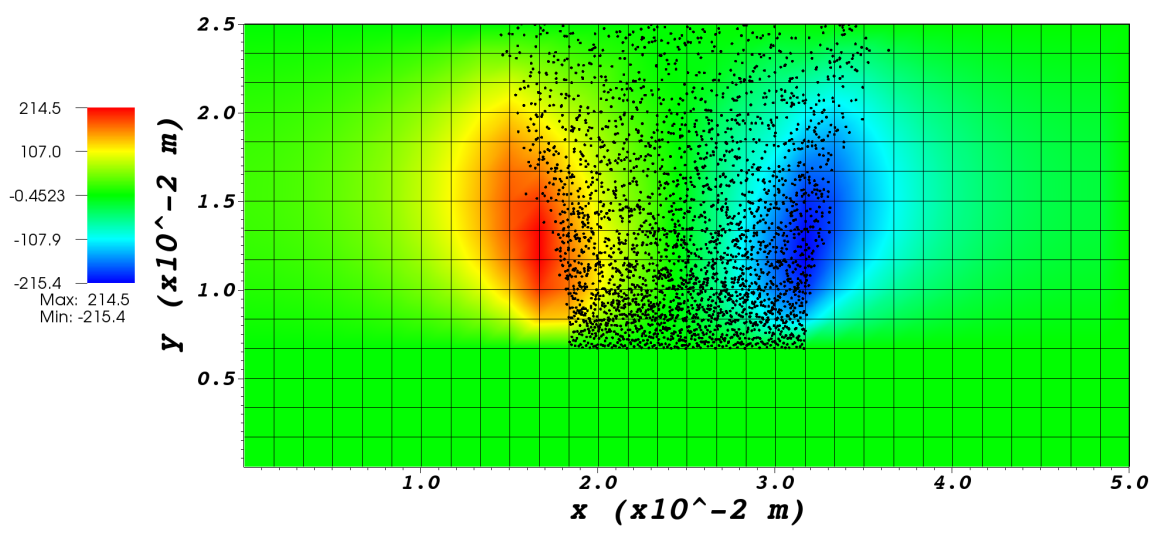

(a)

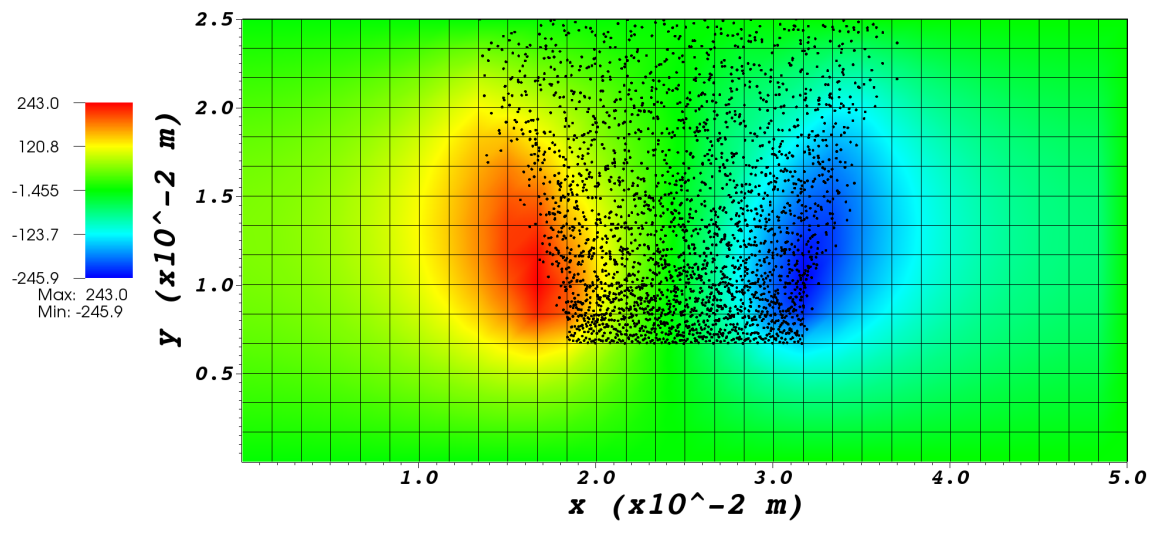

(b)

Figure 5.23: The x-component of the electric field overlaid with electrons (a) in a standard vacuum region and (b) in a divergence free region

This effect limits the use of the approximation to simulations where the beam current is moderately less than the space charge limit. In order to introduce these space charge effects, they must be manually created. One way to do this is to make the electric fields at the point of injection look as if electrons were being emitted from a conductor. In order to approximate the conductor fields, image theory can be 
utilized to pre-load a charge density within the divergence free region which mirrors the expected current in the interaction region. This approach mimics the electron trajectories emitted from a conductor at the emission location. If this works, this would be an improvement, but the actual emission point should be at $y=0$. In order to emit electrons from a location within vacuum and have the trajectories as if they were emitted from a conductor located at $y=0$, the electrons can be emitted with the theoretical energies of electrons emitted from $y=0$. With both the static electric and magnetic fields, this would be difficult and would require an extensive study. These methods were not studied here, and the proposed method was deemed sufficient as emitted current is below the space charge limit.

\subsubsection{Distributed Cathode With Spatial and Time Varying Current}

In addition to just trying different spatial current distributions, spatially-time varying currents can also be used. Spatially-time varying currents have been simulated in magnetrons [13, 93-95] and have shown improvements to efficiency and demonstrated phase control, among other things. For simplicity, in the simulation, discrete emission locations are not used, and the current varies continuously along $x$. To implement this in VSim, the relMacroFluxFunc function is used.

The relMacroFluxFunc is a function whose inputs are spatial and time coordinates, and output is a value from 0-1. Each time there is a particle load attempt, a random number from $0-1$ is generated, and if the random number is less than the value of relMacroFluxFunc, then the particle is loaded; otherwise it is not loaded. This can create non-uniform loading density and variations in time. Note that with a specified number of macroparticles to be emitted per time step and because not all particles are actually emitted, there will be less current actually emitted than what is specified. 
The number of macroparticles per timestep needs to be increased so that the average number of particles emitted yields the desired current.

Four different profiles are studied in this work: two spatial and two time-varying. By varying the spatial profile, the beam injection shape can be tailored to see the effects on gain, efficiency, and noise. The effects on cathode length and the current density vs. length are studied. The time-varying periodic profiles can pre-bunch the beam synchronously with the RF wave to improve gain, efficiency, and noise on the output. These profiles are described in the following sections.

\subsubsection{Spatial Current Profiles}

The two spatial profiles are a uniform profile and a linear profile. The uniform current density profile is constant throughout the cathode, and the total current equals the desired current. Eq. (5.1) describes the uniform profile current density. The total current is $I_{\text {tot }}$ and the sweep variable here is the length of the cathode, $L_{e}$, to find the effect from the length of the cathode. The second profile is a linear profile described by Eq. (5.2) where the terms are added when the slope is positive and subtracted when negative. $f_{D C}$ is the fraction of the current to be emitted uniformly, and $J_{p}$ is the peak current density in $[\mathrm{A} / \mathrm{m}]$ and is chosen to obtain the desired total current. The current densities given here are in $[\mathrm{A} / \mathrm{m}]$ because they vary only in the $x$-direction and the current is uniform in the $z$-direction. Fig. 5.24 shows three different spatial profiles.

$$
J_{e}(x)=\frac{I_{t o t}}{L_{e}} \quad\left[\frac{\mathrm{A}}{\mathrm{m}}\right]
$$




$$
J_{e}(x)=J_{p}\left(f_{D C} \pm \frac{1-f_{D C}}{L_{e}}\left(x-x_{\text {start }}\right)\right) \quad\left[\frac{\mathrm{A}}{\mathrm{m}}\right]
$$

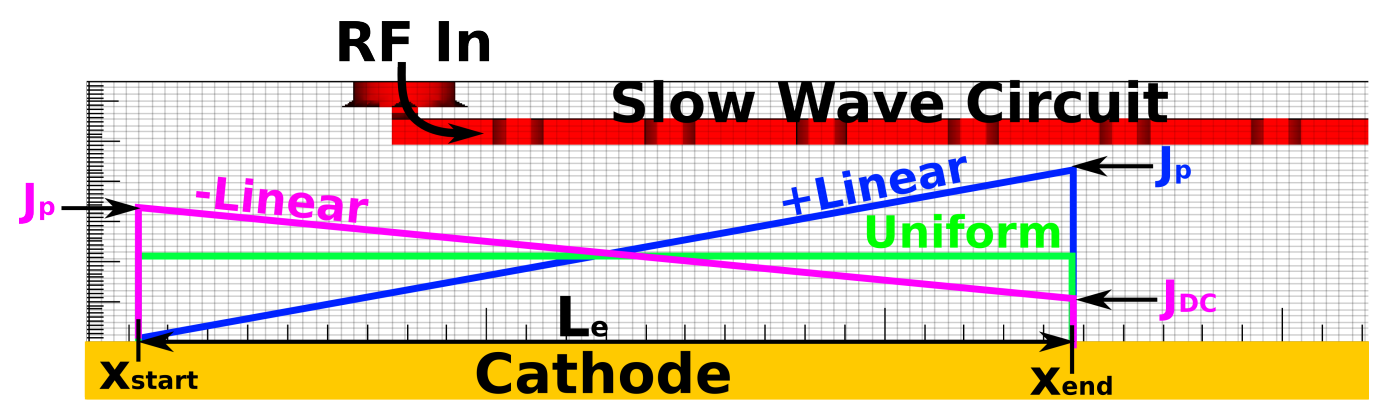

Figure 5.24: Three spatial profiles: linear profile with positive slope and no DC current in blue, linear profiles with negative slope and $50 \%$ DC current in magenta, and a uniform profile in green.

\subsubsection{Sine Wave Emission Profile}

This profile was the easiest time-varying profile to implement and allows for a smooth current density transition from current density peaks to nulls which may have an effect on noise. The sine wave profile is shifted to be between $0-J_{p}$ and travels through time and $x$. Eq. (5.3) shows the simplest form of the equation, with no DC current. $\omega$ is the angular frequency, $\beta$ is the wave number associated with the retarded wave, and $\phi$ is the phase offset used to synchronize maximums in beam currents with minimums of the $x$-component of the electric field. $\phi=\phi_{t}-\phi_{x}+\phi_{90}$, where $\phi_{t}$ accounts for a time offset, $\phi_{x}$ shifts the sinusoid starting point to under the input coax, and $\phi_{90}$ shifts the maximum to be under the input coax. $\phi_{\text {offset }}$ ranges from 0 to $\pi$ and is the controlled phase offset used determine the optimum synchronization between the beam profile and the RF wave. Fig. 5.25 shows a plot of the current distribution compared with the $R F$ wave at $\omega t=\phi_{t}$ with $\phi_{\text {offset }}=0 \mathrm{rad}$. The maximums in the emission profile follow the minimums in the $\mathrm{RF}$ wave with $\phi_{\text {offset }}=0 \mathrm{rad}$. 


$$
J_{e}(x, t)=J_{p}\left(\frac{1}{2}+\frac{1}{2} \sin \left(\beta x-\omega t+\phi+\phi_{\text {offset }}\right)\right) \quad\left[\frac{\mathrm{A}}{\mathrm{m}}\right]
$$

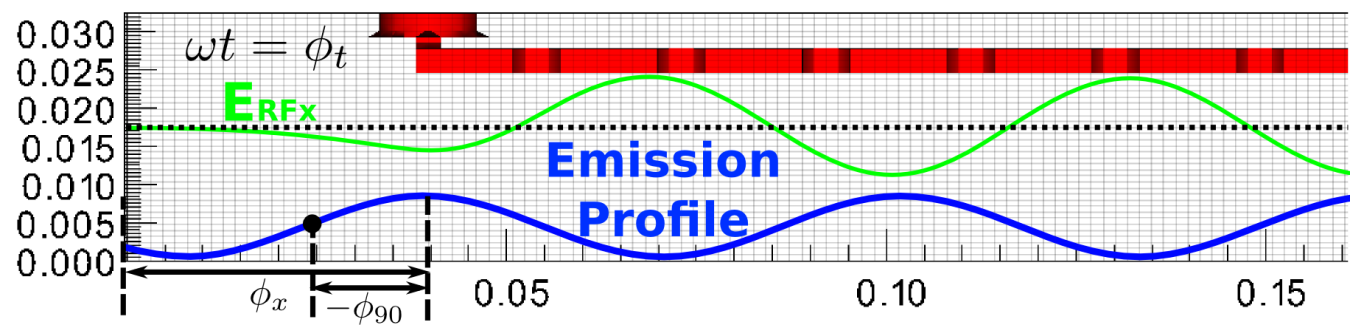

Figure 5.25: The sine wave electron emission profile compared to the $E_{R F x}$ field with $\phi_{o f f s e t}=0 \mathrm{rad}$ at $\omega t=\phi_{t}$. In this case the profile peaks are in the accelerating regions of the $R F$ wave (out of phase).

\subsubsection{Square Pulse Emission Profile}

This profile can vary the current density peaks and can test the effect of synchronous current on the RF wave. It uses square pulses that travel synchronously with the RF wave. This profile is similar to the sine wave profile but has the ability to use varying pulse widths to provide tightly focused beams to better interact with the RF wave on the circuit. The approach is designed to optimize injection of synchronous current. Each pulse can be defined by two Heaviside functions which travel in time as shown in Eq. 5.4. Each pulse would have to be defined explicitly for the entire simulation time. To make it easier, the sine wave function is used in conjunction with the max and ceil functions, shown in eq. (5.5). The function $\max (a, b)$ takes the maximum value $a$ and $b$, ceil $(a)$ rounds up to the nearest integer, and $y_{L p}$ is the $\mathrm{y}$ value corresponding with the desired pulse width $L_{p}$. Using eq. (5.5), all the pulses are defined for all time. Fig. 5.26 shows the square pulse emission profile at $\omega t=\phi_{t}$ compared with the x component of the RF field on the circuit. 


$$
\begin{gathered}
J_{\text {pulse }}=J_{p}\left(H\left(x+\frac{L_{p}}{2}-x_{\text {offset }}-v_{p} t\right)-H\left(x-\frac{L_{p}}{2}-x_{\text {offset }}-v_{p} t\right)\right) \quad\left[\frac{\mathrm{A}}{\mathrm{m}}\right] \\
J_{e}(x, t)=J_{p}\left(\operatorname{ceil}\left(\max \left(y_{L p}, \sin \left(\beta x-\omega t+\phi+\phi_{\text {offset }}\right)-y_{L p}\right)\right)\right) \quad\left[\frac{\mathrm{A}}{\mathrm{m}}\right]
\end{gathered}
$$

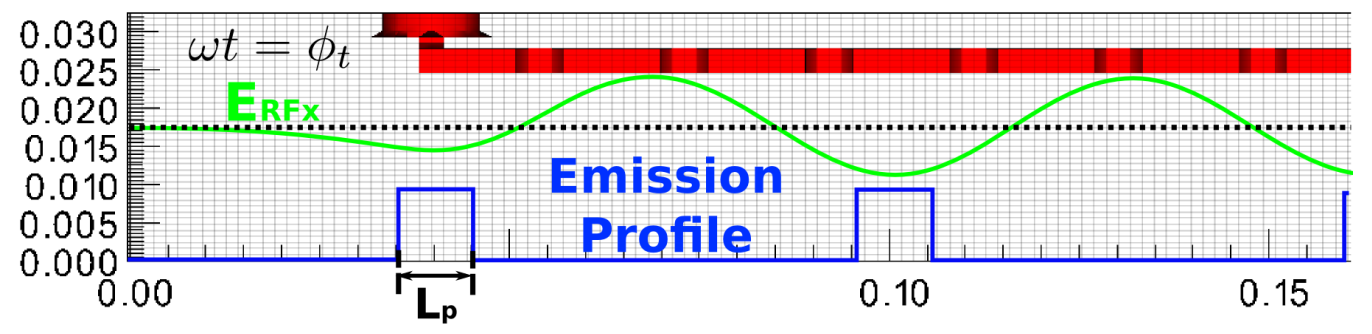

Figure 5.26: The square pulse electron emission profile compared to the $\mathbf{R F}$ electric field in the $x$-direction $\left(E_{R F x}\right)$ at $\omega t=\phi_{t}$. In this case the pulse is "out of phase" with the RF field.

\subsubsection{GFEA Modulated Current Characteristics}

Modulation of the current requires control over when the cathode turns on and emits current and when it turns off. The calculation of GFEA power consumption uses data from the GFEAs studied at Massachusetts Institute of Technology (MIT) [25-27] discussed in Sec. 2.6.3.2. To turn the current mostly off, the gate-to-emitter voltage must be $\lesssim 15 \mathrm{~V}$. To modulate the current from $100 \mathrm{~mA} / \mathrm{cm}^{2}$ to $\sim 0 \mathrm{~mA} / \mathrm{cm}^{2}$, a voltage swing of $30 \mathrm{~V}$ is needed $(15 \mathrm{~V}$ to $45 \mathrm{~V})$. But if "off" was considered to be $1 \%$ of the maximum current $\left(1 \mathrm{~mA} / \mathrm{cm}^{2}\right)$, a voltage swing of only $15 \mathrm{~V}$ is needed. Approximately 
$15 \mathrm{~V}$ is needed to change the current by 2 orders of magnitude at all currents. This reduces the power consumption of the cathode modulation significantly.

To modulate the current, a square gate-to-emitter voltage pulse can be used to turn on and off the current. The GFEA consists of a ground plane with tips and a gate plane above and surrounding the tips separated by a dielectric. This is a capacitive load and can be modeled as a capacitor. To turn on and off the current using a square pulse, the cathode consumes the power as calculated by Eq. (5.6). Using $V_{p p}=15 \mathrm{~V}$, estimating $C=3 \mathrm{nF} / \mathrm{cm}^{2}$, and using the operating frequency of the NU CFA, $f=150 \mathrm{MHz}$, the power consumption of the GFEA is $\approx 50 \mathrm{~W} / \mathrm{cm}^{2}$. The square pulse also limits the upper frequency that can be used, limited by the charge-up time of the capacitor whose cutoff frequency, $f_{c}$, is determined by Eq. (5.7), where $R$ is the series resistance.

$$
\begin{gathered}
P_{g f e a}=\frac{1}{2} C V_{p p}^{2} f \\
\tau_{r c}=R C=\frac{1}{2 \pi f_{c}}
\end{gathered}
$$

Rather than using a square gate-to-emitter pulse to turn on and off the current on the GFEA with minimal power consumption, a sinusoid input signal and a resonant circuit can be used instead. This reduces the bandwidth of the device, but reduces the power consumption dramatically. By using the resonant RLC circuit shown in Fig. 5.27, the load impedance can be designed to be purely real and matched to the transmission line, which is $50 \Omega$. The GFEA is modeled by the capacitance, $C_{g f e a}$, and $L$ and $R$ are the added elements to adjust the load. By sizing $L=1 / \omega_{0}^{2} C$, the reactive impedance is near zero. $R$ is sized such that the real impedance is equal to 
$50 \Omega$ after accounting for the line resistances. The power dissipated into this load is given by Eq. (5.8) where $V_{a m p}=V_{p p} / 2$. Using $V_{p p}=15 \mathrm{~V}$ and $R=50 \Omega$, the power consumption of the GFEA is $\approx 0.5 \mathrm{~W}$. This is much lower power consumption than using a square pulse and is not dependent on the area of the GFEA used since the inductor is sized accordingly.

$$
Z_{L O A D}=R+j \omega L+\frac{1}{j \omega C}=R+\frac{j \omega_{0}}{\omega_{0}^{2} C}-\frac{j}{\omega_{0} C}=R
$$

$$
P_{g f e a}=\frac{1}{2} \frac{V_{a m p}^{2}}{R}
$$

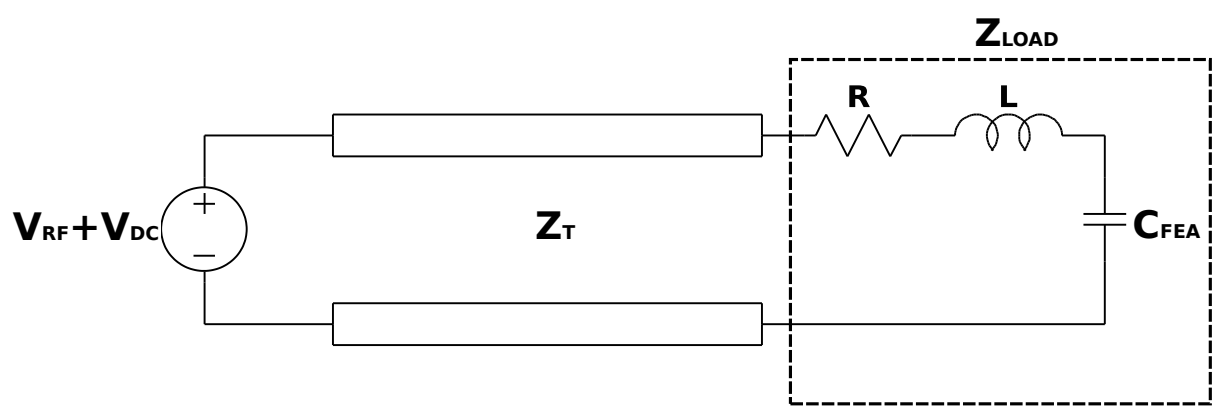

Figure 5.27: Proposed resonant circuit to minimize consumed power by the GFEA.

This resonant circuit would decrease the bandwidth of the device due to the resonant circuit itself and may prove difficult to implement. Another proven design from Calame [97], explained in Sec. 2.8.5, can be used instead. The Calame circuit when used as a resonant circuit, would have drive powers similar to the resonant circuit proposed before $(\approx 0.5 \mathrm{~W})$. When used as a non-resonant circuit to achieve a higher bandwidth, the RF drive power would be comparable to square pulse estimate $(\approx 50 \mathrm{~W})$. 
Emitted electrons when using a sinusoid input signal on the gate-to-emitter input forms a pulse shape due to the exponential I-V relationship shown in Fig. 2.21. The pulse shape for two sinusoidal inputs are shown in Fig. 5.28. The input to the GFEA is sinusoidal with an offset. Fig. 5.28(a) shows a the current density output for a sinusoid which varies from $20-50 \mathrm{~V}$. The pulse width is less than half the period. Changing the sinusoid to vary from $35-50 \mathrm{~V}$ results in the pulse shape shown in Fig. 5.28(b). The pulse width is slightly larger, but maintains the same shape. These current density pulses created by the sinusoidal input are ideal for use in the modulated cathode. The sinusoidal input can turn on and off the current efficiently.

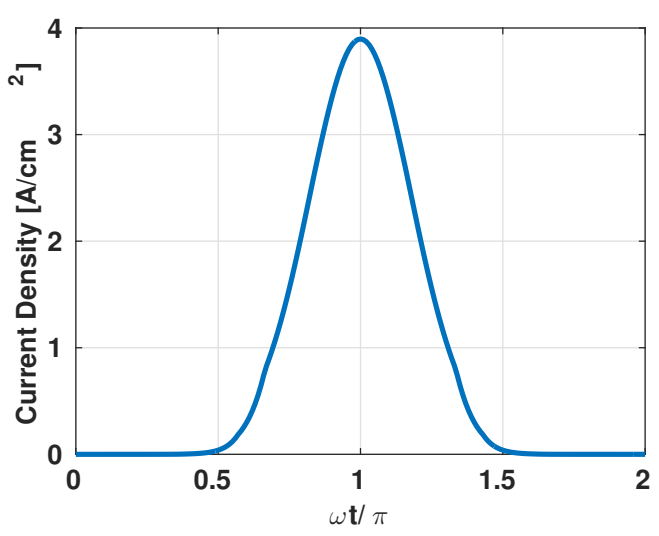

(a)

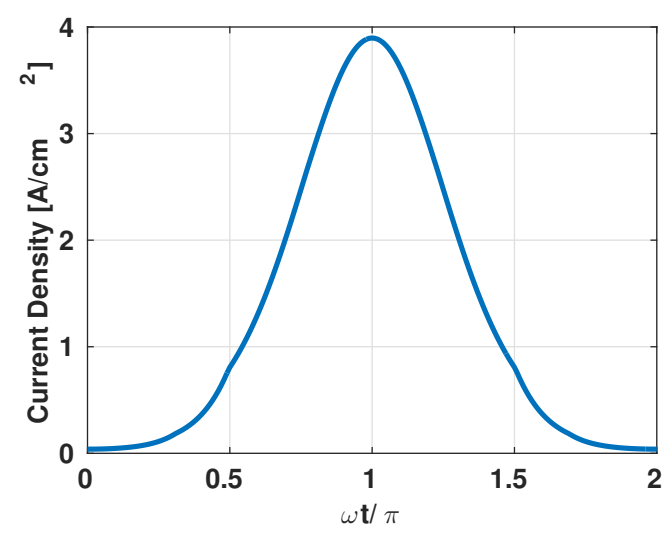

(b)

Figure 5.28: Calculated electron current density pulses from the MIT GFEAs [25] for a sinusoidal input from (a) $V_{g}=20-50 \mathrm{~V}$ and (b) $V_{g}=$ $35-50 \mathrm{~V}$. 


\section{CHAPTER 6}

\section{EXPERIMENTAL AND SIMULATED RESULTS AND DISCUSSION OF BSU CFA}

\subsection{CFA Experiments}

The injected beam experiment at Boise State University using the SW1 and SW2 configuration was performed many times without success. No electron beam interaction with the RF wave was observed. At first, arcing on the cathode was common, and the cathode was constantly damaged. The setup eventually minimized this damaging arcing, and this cathode section functioned for many hours. Low amplitude arcing somewhere else, however, is a constant occurrence at higher voltages, but these arcs do not noticeably damage the cathode. The arcing rate was also observed to increase when the RF wave and electron beam were turned on simultaneously. The reason for the arcing is currently unknown. There are many locations where electrodes are close to each other and have a high potential difference, so pinpointing where the arcing occurs has been difficult.

To determine the reason there was no RF-wave-beam interaction is discussed in the following sections. The potential problems are listed below. The first two potential problems are studied in detail; however the last one is much more difficult to test

with the cathodes available to the group and can only be studied by simulation in 
Vsim.

1. SW circuit dispersion: extensive study on this is provided in Sec. 6.2 and is ruled out as a problem

2. Poor electron optics: extensive study on this is provided in Sec. 6.3 and remains a potential problem

3. Low injected current from the GFEA: simulations results are discussed in Sec. 6.4 and indicate this as the major problem

\subsection{Meander Line Study}

The main goal of these experiments was to find the RF phase velocity at the operating frequency. For brevity, the focus of this will be on SW2. The same analysis was performed on SW1 to determine the predicted circuit retardation.

\subsubsection{S-Parameters}

The S-parameters for SW2 are shown in Fig. 6.1. The plot shows both the simulated (COMSOL) and measured (network analyzer) S-parameters. The plot shows a cutoff region for both simulated and measured results that match reasonably well. The cutoff frequency differers between the simulated and measured results by $200 \mathrm{MHz}$. This difference is discussed in Sec. 6.2.3. 


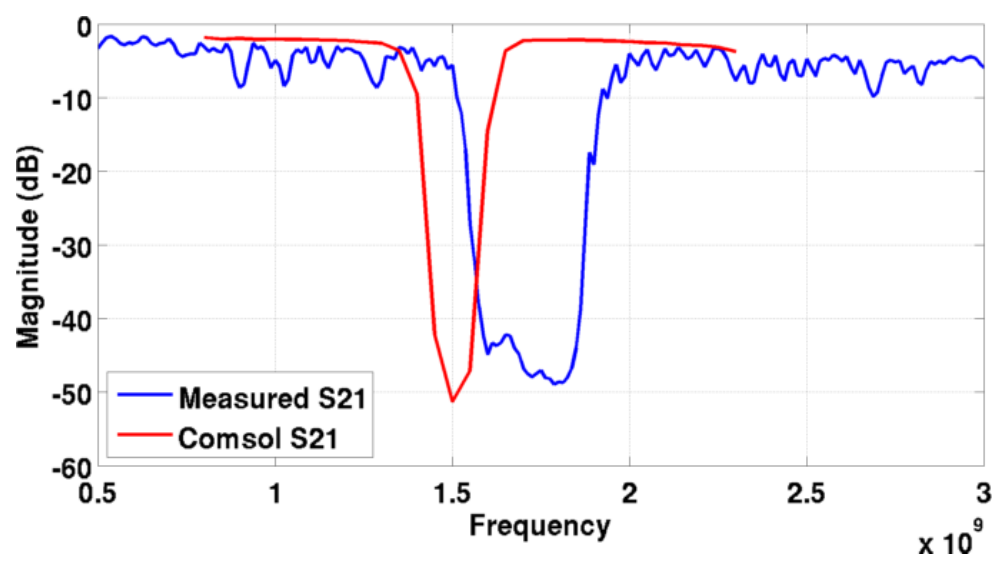

Figure 6.1: S-parameters of SW2 from both simulation (COMSOL) and measured (network analyzer) showing the cutoff frequency.

\subsubsection{Dispersion}

The dispersion was found experimentally and via simulation in COMSOL and Vsim. The dispersion can be determined in many different ways. Two common methods are described in sections 6.2.2.1 and 6.2.2.2. Another common method to determine the dispersion via simulation uses an eiganfrequency solver with periodic boundary conditions, and is well described by McCowen [111]. The experimental method and the COMSOL simulated method use the steady-state standing wave pattern to measure dispersion. The Vsim simulation uses a time-domain method.

\subsubsection{Experimental and COMSOL Method}

The standing wave patterns above the circuit at multiple frequencies were used to determine the dispersion of the circuit. The standing waves are obtained experimentally via the method described in Section 4.2.1.1. COMSOL standing waves were obtained via the method described in Section 5.1. 
To extract the dispersion characteristics from this data, the standing wavelength in the $x$-direction is found using either a $2 \mathrm{D}$ spatial FFT or a $1 \mathrm{D}$ spatial FFT along the center of the meander line in the $x$-direction. From transmission line theory, the traveling wavelength is twice the standing wave wavelength. In this way the wavelength can be determined for each frequency, and a dispersion diagram can be created.

Two examples of the standing wave pattern for the experiment and the simulation are shown for SW2 in Fig. 6.2 and 6.3, respectively. The examples shown are indicative of the range of results. Fig. 6.2(a) shows a frequency where a clean and organized pattern can be observed; whereas Fig. 6.2(b) shows a more 'erratic' pattern. These examples are difficult to interpret by themselves but give a 'feel' for the standing wave pattern. For the lower frequencies, where the slow wave wavelength is greater than two periods of the meander line, the field looks fairly uniform across the $z$-axis. At higher frequencies, where the wavelength is shorter then two periods of the meander line, the edges of the circuit have higher electric field intensity than the center.

To determine the wavelength, a 2D FFT is used to find the frequency components in the $x$-direction. Fig. 6.4 shows the $x$-component of the $2 \mathrm{D}$ spatial FFT of the experimental and simulated electric fields for two frequencies. For the case where $f=1.15 \mathrm{GHz}$ there are multiple wavelength components. Two of which are the forward wave and the backward wave, and a third one is the wavelength component due to the pitch of the circuit. The backward wave for the higher frequencies is mainly due to the high electric fields on the edges of the circuit. To determine if the wavelength component is a backward or forward wave, the peak is monitored as the frequency is increased. As the frequency is increased, a forward wave wavelength gets 
smaller and moves to the right in the figure. A backward wave wavelength increases and moves to the left in the figure. The pitch component is observed for many of the FFTs generated and does not move as the frequency is increased.

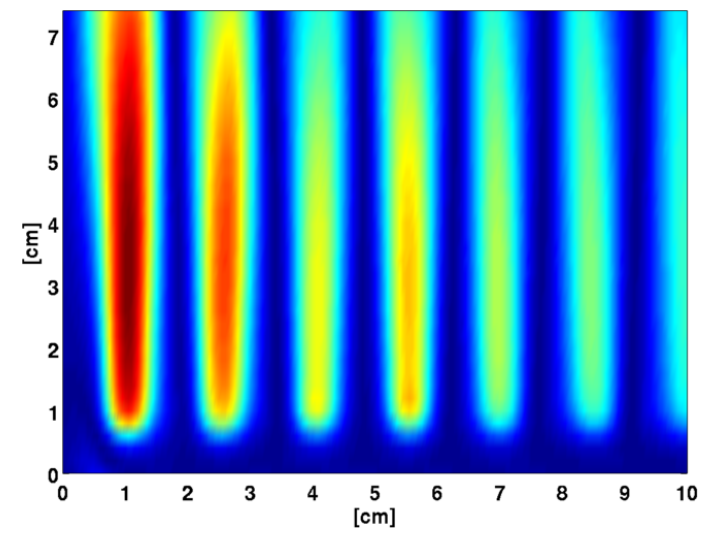

(a)

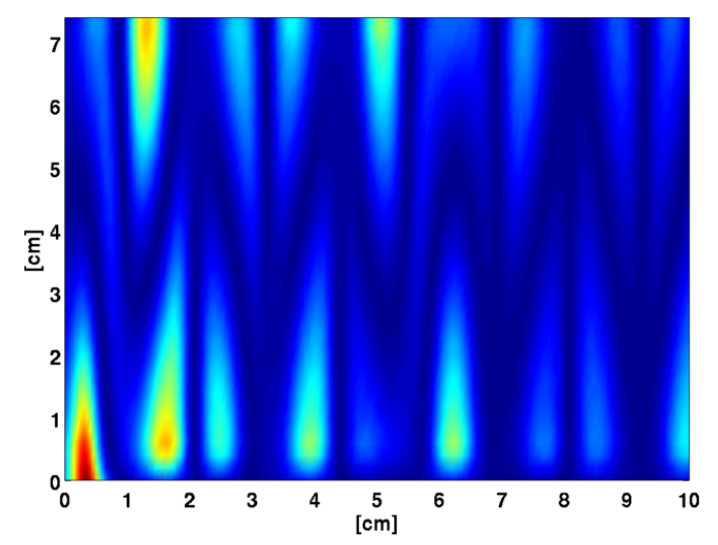

(b)

Figure 6.2: Measured electric field intensity for SW2 for frequencies (a) $500 \mathrm{MHz}$ and (b) $1.15 \mathrm{GHz}$.

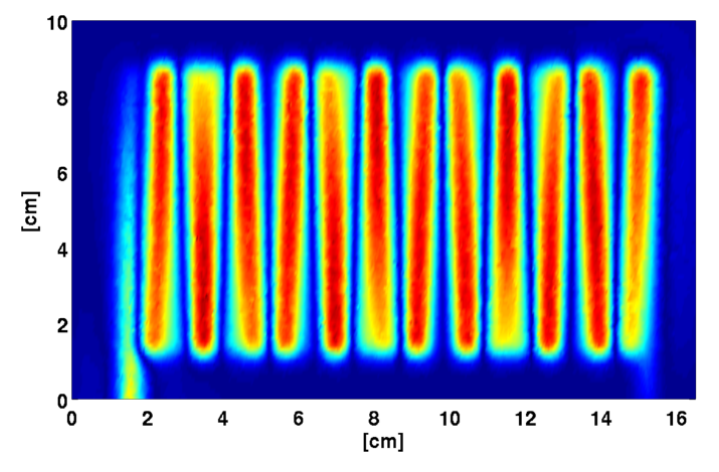

(a)

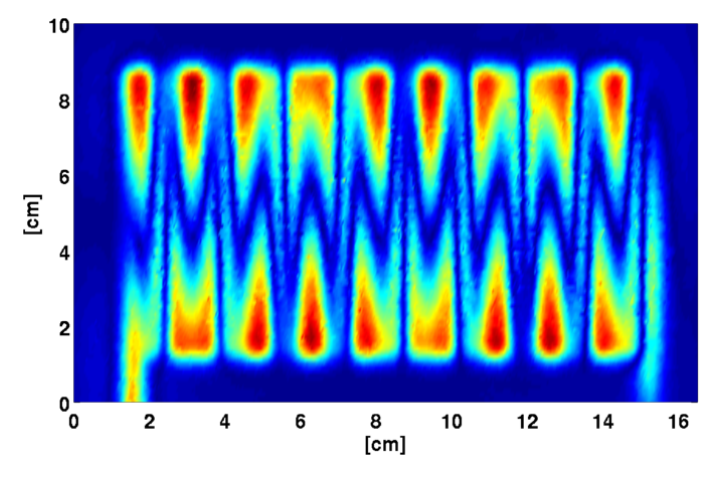

(b)

Figure 6.3: COMSOL simulated electric field intensity for SW2 for frequencies (a) $500 \mathrm{MHz}$ and (b) $1.15 \mathrm{GHz}$ 


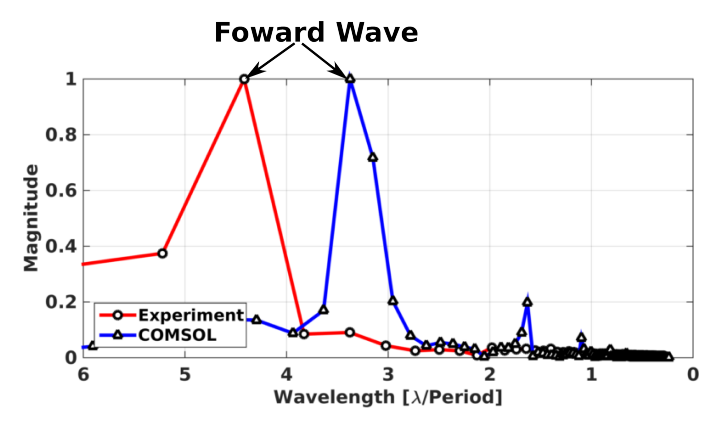

(a)

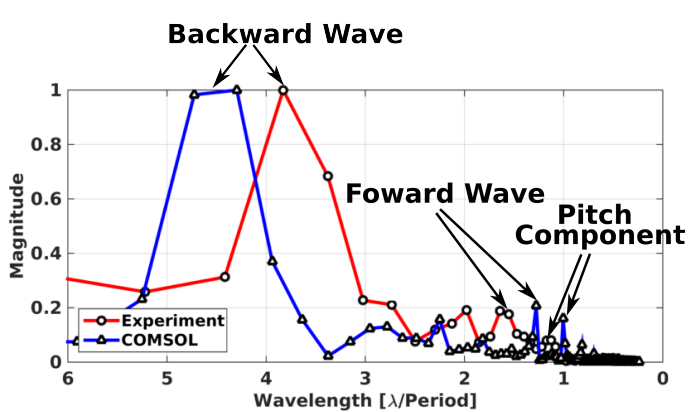

(b)

Figure 6.4: 2D spatial FFT of the measured and simulated electric field intensity for SW2 for frequencies (a) $500 \mathrm{MHz}$ and (b) $1.15 \mathrm{GHz}$.

\subsubsection{VSim Method}

This method can obtain much of the dispersion digram in one long simulation. One way is to run many simulations at each frequency and measure the wavelength of each simulation, but this would take a lot of simulation time. The single simulation method uses periodic boundaries and an impulse signal get the dispersion curve up to the first cutoff frequency. The model uses $\mathrm{N}$ pitches of the slow wave circuit which goes all the way to the edge of the domain in $x$ and is essentially continuous from one side to the other. A signal traveling down the circuit from one side goes into the periodic boundary and continues traveling on the other side of the domain. A higher number of periods used in the domain allows measurement of the lower the frequency components. An impulse is excited within the dielectric of the SW circuit in between the metal of the ground plane and the circuit. The pulse is created by writing a high frequency pulse (ten times the operation frequency of the circuit) directly to the $y$-component of the electric fields to a location within the dielectric. This pulse travels down the circuit in both directions and continuously travels forever within the domain due to the periodic boundary conditions. 
The voltage is measured outside the dielectric from the meander line to the edge of the domain. An example of the measured voltage signal for SW2 using 10 slow wave circuit pitches is shown in Fig. 6.5(a). This signal in the time domain looks like noise, but taking the FFT of it shows that the noise consists of discrete frequencies. The FFT is shown in Fig. 6.5(b). These frequency components are the modes of the circuit, and using Eq. (6.1), the wavenumber $k$ can be determined which in this case is equal to the phase constant $\beta$. Note that the frequency components are almost equally spaced from 0 to $1.3 \mathrm{GHz}$, and the spacing between the $1.3 \mathrm{GHz}$ and $1.5 \mathrm{GHz}$ peaks are slightly larger. This larger spacing corresponds to cutoff. Another important thing to note is that the placement of the voltage diagnostic in $x$ is very important. At some locations, certain modes can be missed. The reason for these missed modes is speculated to be due to a null in the standing wave pattern of that mode. By shifting the location slightly in one direction or the other, the mode reappears.

$$
k=\frac{2 \pi}{L} n
$$




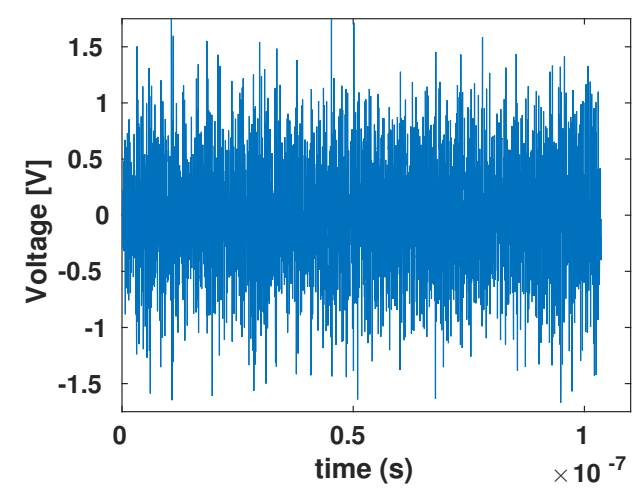

(a)

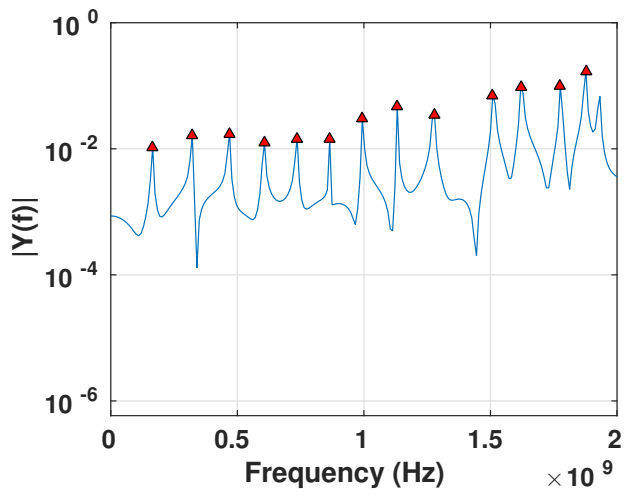

(b)

Figure 6.5: (a) Voltage through time of the VSim dispersion model using an impulse signal and periodic boundaries in $x$, and (b) the FFT of that signal.

\subsubsection{Results}

Two different dispersion diagrams are displayed in Fig. 6.6; using the COMSOL and experimental method, one is generated from the $x$-component of the $2 \mathrm{D}$ spatial FFT, shown in Fig. 6.6(a), and the other is generated from the spatial FFT only along the center of the circuit in the $x$-direction, shown in Fig. 6.6(b). The VSim result is plotted alongside the COMSOL and experimental methods using the FFT along the center. The corresponding retardations for of the dispersion on the center of the circuit is shown in Fig. 6.7. The 'jagged' edges in the dispersion and retardation plot for the COMSOL and experimental cases are caused by the resolution of the FFT. The reason for the two plots is to differentiate between the backward waves which dominate the edge of the circuit from the forward waves which dominate the center of the circuit. 


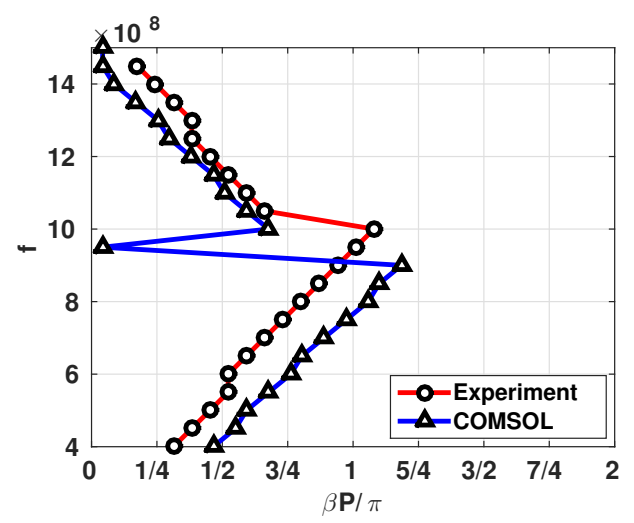

(a)

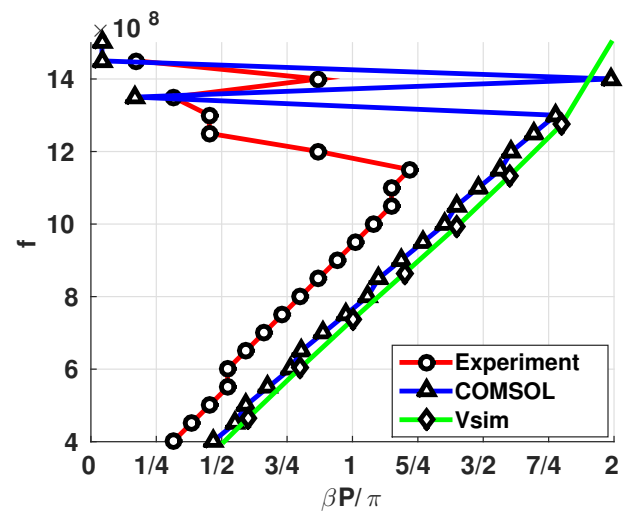

(b)

Figure 6.6: Measured and Simulated dispersion diagram for SW2 using (a) $x$-component of the spatial FFT, and using (b) the FFT along the center in the $x$-direction.

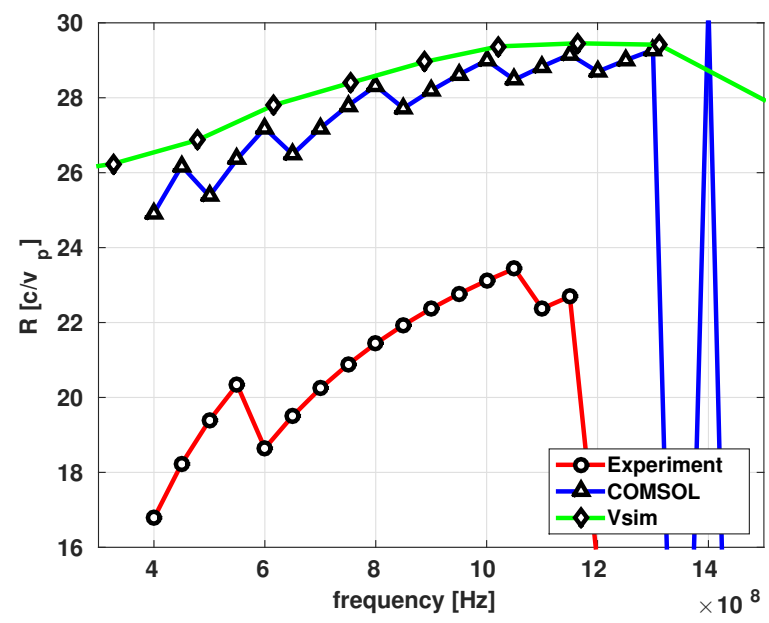

Figure 6.7: Measured and simulated retardations of SW2 using the FFT along the center in the $x$-direction.

The linear portion of the dispersion curves are portions which usually have a clear dominate wavelength. The regions between the positively sloping and negative sloping parts have erratic behavior. This erratic behavior is usually caused by not having a clear dominant wavelength. These transition regions are associated with competing 
wavelength components or could be at the cutoff region where no wave propagation is observed.

For SW2 at $600 \mathrm{MHz}$, the experimental retardation of the phase velocity is found to be approximately 18-20 in the forward wave region. This value allows for a testable CFA setup with the equipment available to the group. To match the beam velocity with the phase velocity of the RF wave and to be between the Hartree and Hull cutoff voltage, voltages less than $5 \mathrm{kV}$ can be used; this voltage is within the capability of the CFA experiment.

For SW1 the retardation of the phase velocity is found to be approximately 10 in the forward wave region. For a beam operating point to be between the Hartree and Hull cutoff voltage, voltages greater than $5 \mathrm{kV}$ are needed, which is outside the capabilities of the CFA experiment.

\subsubsection{Discussion}

The standing wave patterns between simulation and experiment seem to match reasonably well. At low frequencies $(0.4-0.9 \mathrm{GHz})$, the standing wave pattern is constant throughout the width of the circuit. At higher frequencies, the power is higher towards the edges.

The dispersion curves of VSim and COMSOL simulations in Fig. 6.6 match simple theory fairly well. On the edge of the circuit, which the dispersion curve in Fig. 6.6(a) captures, the backward wave should begin at $\beta P / \pi=1$, which it does for both experiment and simulation as indicated by the negative slope. In the center of the circuit, which is shown in Fig. 6.6(a), cutoff should begin close to $\beta P / \pi=2$, which it does in the simulation at around $1.4 \mathrm{GHz}$. The cutoff observed in the dispersion diagram of the center fields also matches the simulated $S_{21}$ parameters shown in Fig. 
6.1. The experimental cutoff in the experimental dispersion diagram down the center of the circuit is difficult to see. The forward wave is weak in the center and has many unexpected wavelength components; whereas the edge of the circuit has a relatively clean dominant backward wave mode. According to the $S_{21}$ parameters shown in Fig. 6.1, cutoff of the experimental circuit should begin at $1.5 \mathrm{GHz}$. By extending the experimental dispersion curve line to $\beta P / \pi=2$, the estimated cutoff would be about $1.5 \mathrm{GHz}$ which matches the $S_{21}$ parameters.

The dispersion plots between simulation and experiment do not actually match very well. The retardations observed for the experiment range from 17-22 while the simulation ranges from 25-30. A similar difference was also observed from SW1, where the retardations for experiment and simulation were 10-13 and 13-16, respectively. The experimental retardation is always smaller than the simulated. One source of error is the difference in the pitch from simulation to experiment. Note that the FFT plot in Fig. 6.4 shows a difference in the pitch component wavelength. The pitch component for COMSOL lies directly at $1 \lambda /$ period, which corresponds to a $7 \mathrm{~mm}$ wavelength since it is normalized to this pitch. The experimental pitch component is at $1.1 \lambda /$ periodwhich corresponds to $7.7 \mathrm{~mm}$ wavelength, which indicates the actual pitch of the circuit is slightly larger than the COMSOL pitch. This difference lowers the estimated retardation of the experiment from 30 to 27.5 , which is closer to the measured retardation of 17-22. This change lowers the error but does not account for all of it.

The reason for the difference in retardation is unknown. One major difference between the simulation and the experiment are the Teflon screws used in the experiment, which are not modeled in simulation. The Teflon screws, however, would probably increase the retardation due to the increased area of a dielectric around the 
circuit. Also, the Teflon screws are similar to the dielectric supports used in helix slow wave structures, which are shown to increase the retardation. The most probable reasons are manufacturing defects in the meander line or errors in the geometry due to hand construction of the slow wave structure. It is believed that the simulation is correct, but has different geometry than the actual experimental circuit. The circuit varies in alignment and pitch slightly at many locations compared to an exactly rendered circuit in the simulations. The structural difference is unknown, and due to time constraints and with the lack of interaction observed with the full experimental setup, investigation of SW2 was discontinued.

Another observation is that the retardation increases as the frequency is increased. Also, the estimated retardation corresponds well for frequencies right before cutoff. The estimated retardation using Eq. 2.5 for SW2 is 30, and the simulated retardation is 30 right before cutoff. This is also observed for SW1, not shown here, SW3, shown in the next chapter, and for meander lines in [48]. The reason for the lower retardation at lower frequencies is due to a decrease in inductance as the frequency is decreased, which increases the phase velocity. An explanation for why the inductance decreases with frequency for the for the helical slow wave circuit is provided in Gilmour [2], and similar arguments can be made to the meander line.

Even though the experimental retardation could not be directly confirmed with simulation, the general trends are confirmed. The increase in retardation with frequency, the backward waves along the edges of the circuit, and the cutoff point are all correctly modeled. Also, the COMSOL method matched very closely to the VSim model. Even though the magnitude of the retardation did not match, these other factors confirm the validity of the VSim simulation model, and the magnitude difference is due to spatial discrepancies with the experimental slow wave circuit 
geometry and are not a simulation problem.

\subsection{Beam Optics: SIMION Comparison With Experiment}

The electron beam in the CFA was experimentally characterized and compared with the SIMION simulation. The injected beam CFA configuration was studied to ensure proper beam formation for proper beam interaction with the RF beam. With constant electrode potentials, the currents were measured with respect to the applied B-field. This experiment determines the Hull cutoff condition and the current distribution along the tube.

\subsubsection{Experiment}

The CFA setup is shown in Fig. 4.2 in Section 4.1.2. Each of the resistors in the diagram are current sense resistors. The emitted current is monitored using $R_{c}$, which actually monitors the cathode power supply current. Only emitted current that manages to go to the anode, end collector, or any conductor at earth ground (the chamber wall for example) will be registered on $R_{c}$. Any emitted current that collects on the electrodes on CFA ground will not be registered. This method of determining emitted current turns out to be a good approximation and is very informative.

Visual cues can also be used to determine where the current is going. When electrons collide and give up their energy to an electrode, photons are emitted. Enough photons are emitted to be registered by eye, and using this light, one can determine where on the electrode the beam collides. This is particularly helpful for determining where on the end collector the beam collides. 


\subsubsection{SIMION}

The CFA setup is shown in Fig. 5.2 in Section 5.2. The currents are calculated on the End Collector, Anode, and Gate in SIMION. The current is the number of trajectories that collect on the electrode, rather than in actual charge.

\subsubsection{Results}

These results correspond to a anode-sole potential of $3000 \mathrm{~V}$. The anode-sole gap is $10 \mathrm{~mm}$. These results are only discussed briefly for brevity.

\subsubsection{Experimental}

The I-B curve is shown in Fig. 6.8. The total current is the sum of the current collected on the anode and the end collector. The end hats and sole collect no current and are not shown. The Hull cutoff condition, where current collected on the anode transitions to being collected on the end collector, occurs from $15-20 \mathrm{mT}$. The large fluctuations of end collector current in Fig. 6.8 for B-fields $>27 \mathrm{mT}$ are not noise or measurement error and are considered a real phenomenon. 


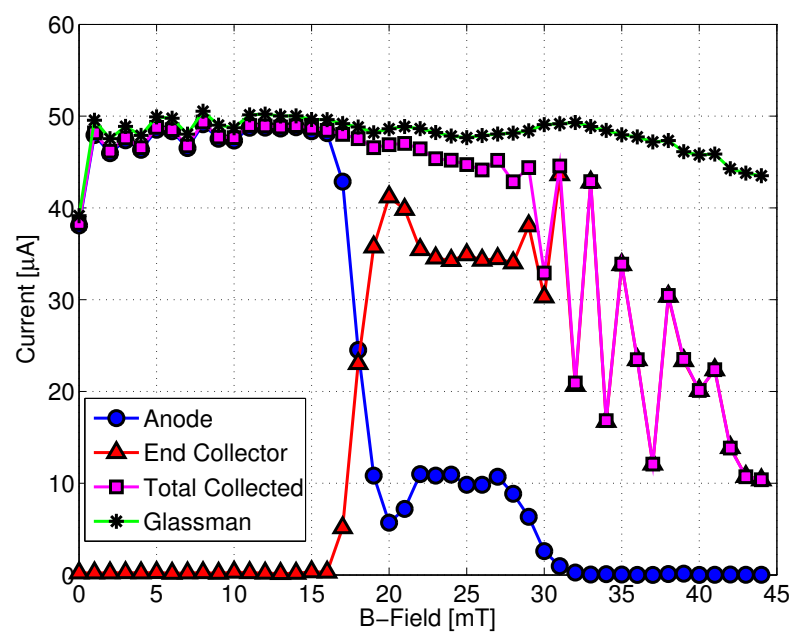

Figure 6.8: Experimental I-B Curves

\subsubsection{Simulation}

The I-B curve is shown in Fig. 6.9. The Hull cutoff condition occurs from $10-20 \mathrm{mT}$.

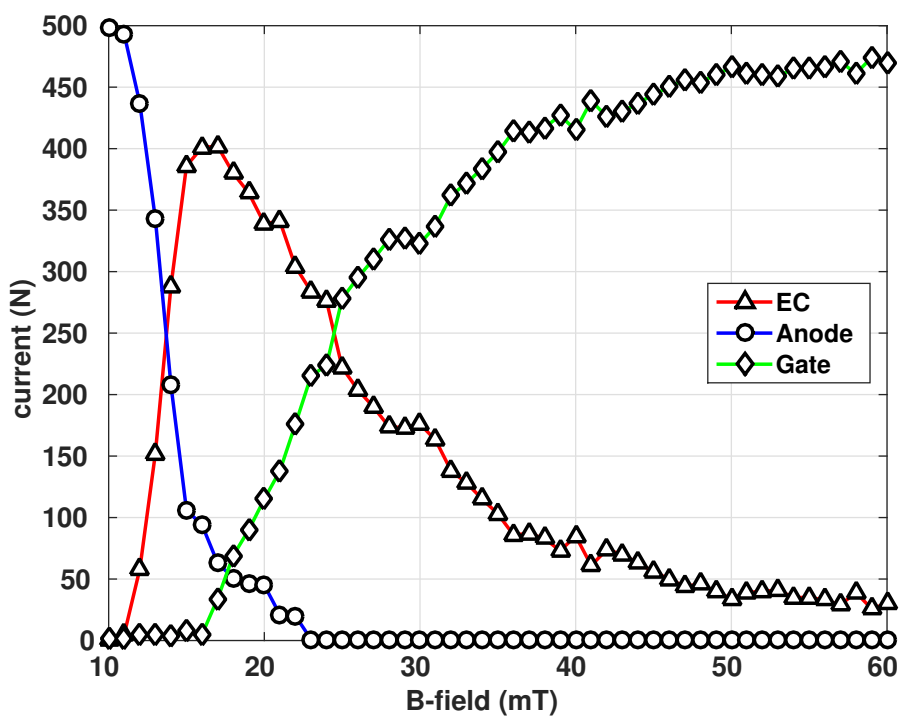

Figure 6.9: SIMION I-B Curves

Some example images of the trajectories. The black lines represent the electron trajectories. The red dots are "splats," when the electron is collected. The smaller 
grid cells are the conductor boundaries. See Fig. 5.2 to better interpret the conductor boundaries.

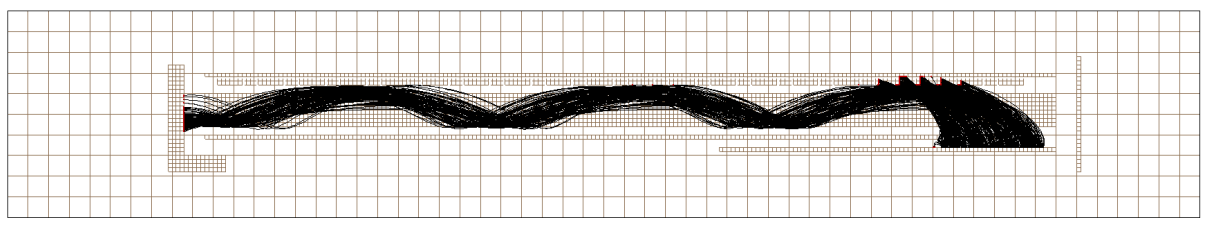

(a) $125 \mathrm{mT}$

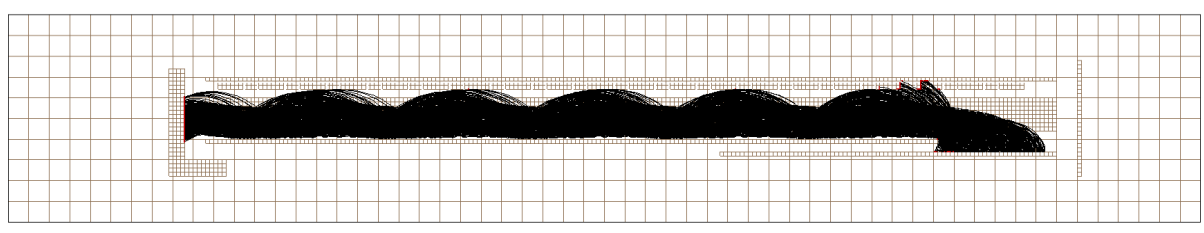

(b) $175 \mathrm{mT}$

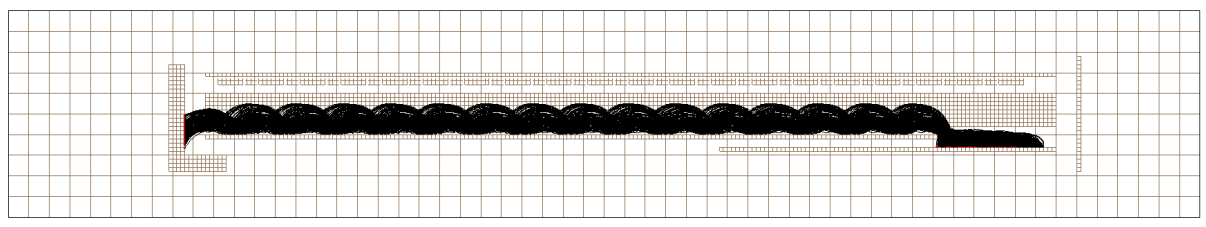

(c) $300 \mathrm{mT}$

Figure 6.10: Sample Trajectories for three different magnetic fields. With lower magnetic fields, more electron trajectories collect on the slow wave circuit, at high magnetic fields, most of the current travels down the tube to the end collector.

\subsubsection{Discussion}

The calculated magnetic Hull cutoff for this configuration is $20 \mathrm{mT}$. These experiments seem to correlate well with this. The fact that the transition of current from the anode to the end collector occurs over $5 \mathrm{mT}$ is to be expected. The Hull cutoff is the condition derived for electrons born with $0 \mathrm{eV}$ initial energy. The GFEA emits electrons with a distribution of energies which will smooth the transition. 
The main difference between the I-B Curves of the experimental and simulated results is the larger gate current for the simulated case. The large gate current is to be expected because of the initial kinetic energy of the electrons allowing them to easily collect on the gate. There is no direct measurement of the gate current current in the experiment due to the large leakage current; however the Glassman high voltage (HV) power supply current gives an estimate of the emitted gate current. The HV current measures all the current from the high voltage to earth ground. Any current that deviates from the anode, end collector, or the chamber wall to the gate will decrease the Glassman current. The HV current only decreases by $\sim 5 \%$ at the highest B field, so the emitted current cannot be returning to the gate. The simulated currents show the majority of the electrons at the highest B-field return to the gate. The reason this does not occur in the experiment is currently unknown. The unexpected measurements in beam current indicate that the electron beam is not behaving as expected and may be a reason for the lack of interaction with the RF wave.

Another main difference is that current is 'lost' in the experiment at higher magnetic fields. As indicated by the relatively constant HV current, the beam still manages to go to earth ground, however, not to any electrode being measured. It is assumed to be current going to the chamber wall. How the current makes its way to the chamber wall is unknown.

There are additional peculiarities in the experimental curves such as the "hump" at $20 \mathrm{mT}$. This effect might be recreated in the simulated curve where there is a flat region, where the currents are stable for a few data points, at around $20 \mathrm{mT}$. The reason for this hump is peculiar and the origin is unknown. The wide variations in end collector current at magnetic fields greater than $27 \mathrm{mT}$ are also peculiar and consistently repeatable. Their origin is also unknown. 
Another phenomenon that is not shown in the presented data is the current drifts in the z-direction. The photons emitted from the end collector indicate that all the current is collected on one side. This was also observed from the phosphor screen experiments performed (also not given here). As some electrons of the cycloiding beam graze the phosphor screen, the beam was observed to drift a little in the direction of the magnetic field. Tilting the magnetic field by physically angling the electromagnets was observed to counteract this drift. More experiments and quantitative study needs to be performed to determine the cause. The possibilities are that the magnetic field may not be correctly aligned or that the beam acquires some $z$-velocity component from emission or the electric field. Preliminary experiments tilting the magnetic field in SIMION show different behavior than observed in the experiment, but no conclusions can be made.

\subsection{Vsim Simulations}

A close approximation of the CFA configuration which uses SW2 was created in Vsim. Because the experiment never showed any beam interaction with the RF wave, the goal was to corroborate this observation and to determine the reason for this lack of interaction. As described in the next chapter on SW3, it was found that the beam injection affects the gain greatly and requires extensive optimization. A similar optimization technique was used on SW2, but the only parameters optimized with the SW2 model was $V_{c s}, V_{a s}$ and $B$. Also, it was found that low currents

comparable to the ones used in the experiment $\left(I_{\text {beam }}=5 \mathrm{~mA}\right)$ yielded no gain, and a much greater current was needed, $\left(I_{\text {beam }}=150 \mathrm{~mA}\right)$. With $I_{\text {beam }}=150 \mathrm{~mA}$ and the optimum parameters $V_{a s}=1700 \mathrm{~V}, V_{c s}=300 \mathrm{~V}, B=15.4 \mathrm{mT}$, and $I_{\text {beam }}=150 \mathrm{~mA}$, 
$P_{r f}=1 \mathrm{~W}$, a gain of $2.2 \mathrm{~dB}$ was observed. Figure 6.11 shows the VSim model and the corresponding electron trajectories.

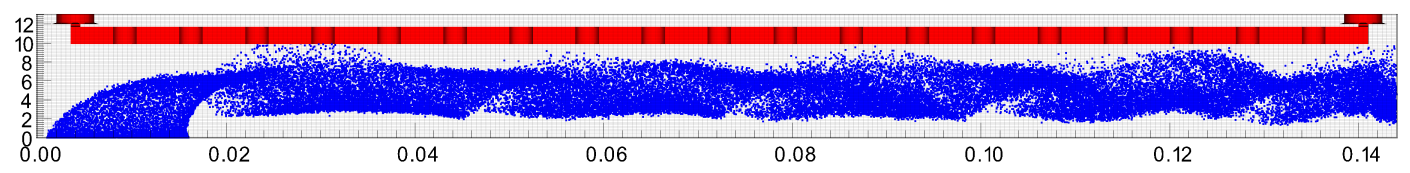

Figure 6.11: The SW2 model used in VSim with electrons shown in blue and the slow wave circuit in red

The reason for the low gain is due to the poor interaction impedance of the $\mathrm{SW}$ circuit. The interaction impedance relates the RF input power to the electric field observed in the interaction region. A higher interaction impedance means higher electric fields in the interaction region, which increases the RF wave-beam interaction, which increases the gain. The interaction impedance is found by running the simulation with the RF input and no particles, then measuring the $x$ - and $y$-components of the electric field amplitude along the interaction region at the beam location, and using Eq. 2.11 in Sec. 2.5.1 with any RF input power. The interaction impedance for SW2 is found to be only $2.5 \Omega$. To visualize the poor interaction on the device, the gain along the length of the circuit is shown in Fig. 6.12 for a circuit 1.5 times longer than SW2 at the optimum parameters. The results for the longer circuit show slope of the gain and make it easier to compare with the estimated gain from Pierce theory, summarized in Sec. 2.5. Figure 6.12 also shows the theoretical gain predicted by Pierce theory for beam currents of $I_{\text {beam }}=20 \mathrm{~mA}$ and $150 \mathrm{~mA}$. The gain is determined by monitoring the voltage and current on the circuit. More details on the gain calculations are discussed in Sec. 7.4.3.2. 


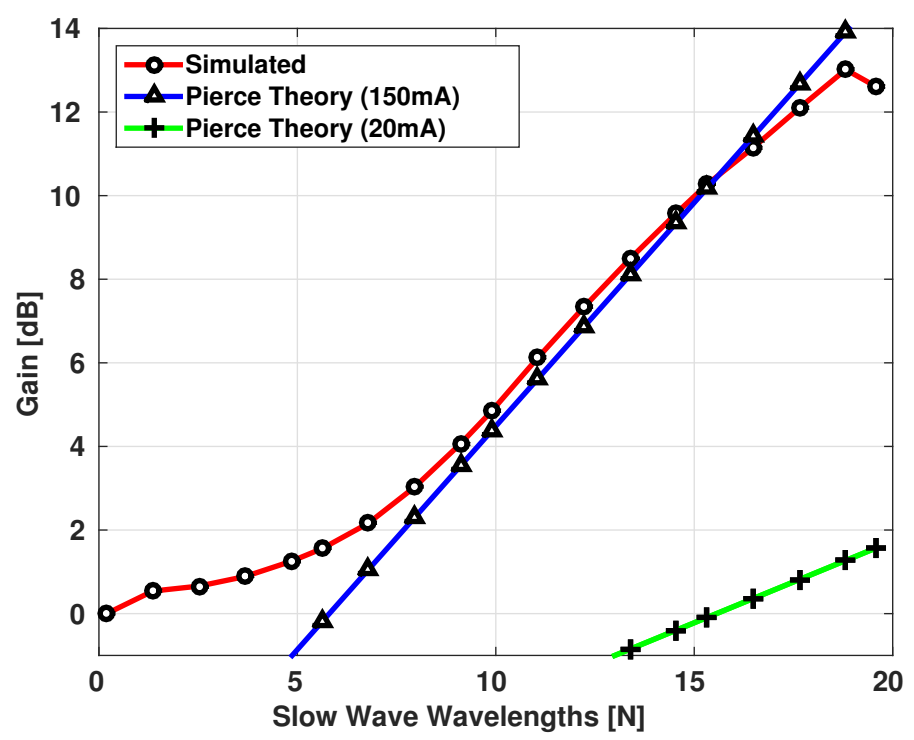

Figure 6.12: Gain along the length of the SW2 circuit compared to the predicted gain of Pierce theory for $P_{r f}=1 \mathrm{~W}, I_{\text {beam }}=20 \mathrm{~mA}$ and $150 \mathrm{~mA}$, and a circuit whose length is 2.5 times as long as the experimental circuit.

The simulated gain steadily increases along the length of the circuit, with a small dip at the end of the circuit. The gain at the beginning and end of the circuit is measured in the coax circuits; whereas the other data points are measured on the microstrip line itself. The dip at the end is thought to be because of a mismatch going from microstrip to the coax cable. The simulated gain follows the theoretical gain predicted by Pierce theory for $I_{\text {beam }}=150 \mathrm{~mA}$ really well. Note that the experimental slow wave circuit is only about 6 slow wave wavelengths long, and the gain at that point matches the $1.5 \mathrm{~dB}$ gain observed in the simulation shown in Fig. 6.11. Even with a small interaction impedance, the device can still extract energy from an electron beam, but it requires much more current than originally thought.

The predicted gain for $I_{\text {beam }}=20 \mathrm{~mA}$ is shown in Fig. 6.12 and is only $2 \mathrm{~dB}$ for a long circuit length, and no gain is predicted for the experimental circuit length of 6 
slow wave wavelengths. The actual currents used in the experiment were $5 \mathrm{~mA}$, and the gain predicted for that are so low that they do not show up in the plot. These results show that the CFA configuration using SW2 with currents less than $100 \mathrm{~mA}$ would never show any interaction with the electron beam. Hence, the simulation demonstrates the problem with the experiment.

\subsection{Summary}

The experimental observations were all corroborated by simulation. The experimental electron beam characteristics were measured and confirmed by SIMION simulation. There were a few peculiar experimental beam anomalies the could not be explained, but these anomalies are not thought to cause the lack of interaction. The dispersion characteristics were experimentally measured and compared with COMSOL and VSim simulations. The experimental dispersion trends are confirmed by simulation; however the experimental retardation magnitude was $22 \%$ less than the simulated retardation at $600 \mathrm{MHz}$. The difference in magnitude is thought to be caused by the difference in the fabricated circuit vs. the simulated circuit. While this magnitude difference is large, the general behavior such as cutoff, backward waves on the edge of the circuit, retardation increasing with frequency, and the behavior of the electric field above the circuit, are all confirmed by simulation. Even though there is an unknown mismatch between the simulation and experimental model, because the general trends are conserved and the model is close, the VSim simulated model can be used to draw conclusions about SW2.

The experiment never showed any RF interaction with the electron beam which is confirmed by the VSim simulations and theory. The simulations and Pierce theory 
both show that a minimum of $150 \mathrm{~mA}$ beam current was needed to show appreciable gain, and the maximum current used in the experiment was only $5 \mathrm{~mA}$. The low gain of SW2 is attributed to the low interaction impedance of the meander line circuit and the low beam currents.

With no appreciable experimental results on SW2, the focus turned to a new CFA using the meander line circuit, SW3. This circuit was used experimentally at Northeastern University [14, 15] and showed gain. Using the experimental data from that work, the VSim model could be validated. The next chapter outlines the simulation work performed on SW3. 


\section{CHAPTER 7}

\section{EXPERIMENTAL AND SIMULATED RESULTS AND DISCUSSION OF NORTHEASTERN UNIVERSITY CFA}

Because no gain was observed for SW1 and SW2, the design was shifted to a different design used in a CFA at Northeastern University [14, 15], described in Sec. 2.8.3. This work observed gain in their CFA experiment and provides many different types of measurements. These experimental results can be compared with the simulations performed in this dissertation to validate the model. All the simulations and experiments discussed in this chapter are for the Northeastern design, called here SW3.

The NU CFA using the SW3 slow wave circuit was modeled in Vsim. The circuit and configuration is very similar to the BSU CFA using SW1 and SW2, and the same simulation model was used but with the new dimensions. One difference between the experimental SW3 and the simulated one is that the experiment uses a copper tube with a circular cross-section for the meander line; whereas the simulation uses a rectangular cross-section. This adaptation was used to reduce the resolution requirements and to decrease simulation time.

\subsection{Dispersion}

The dispersion of the actual circuit was never measured, but the dispersion of the simulated circuit is shown here. Fig. 7.1 shows both the dispersion and the retar- 
dation vs. frequency of SW3. These plots cannot be directly compared with actual results but can be compared with the reported retardation at the operating frequency of $150 \mathrm{MHz}$. At the operating point of $150 \mathrm{MHz}$, the simulated retardation is about 32 and the experimental retardation is 31 . These values are very close, and the slight error can be explained by imperfections and geometric variation in the experimental setup. These imperfections can be due to error in the pitch, the Teflon screws, which are not modeled in simulation, the flatness of the circuit, unwanted bends, and other variations. Also, the "measured" retardation is inferred from the $\mathbf{E} \times \mathbf{B}$ velocity which gives the maximum gain. Error in the anode-sole distance would shift the actual retardation. The dispersion plots are also helpful in confirming the bandwidth of the circuit. The retardation varies over this frequency range and goes from 31 to 33 from 100 to $200 \mathrm{MHz}$.

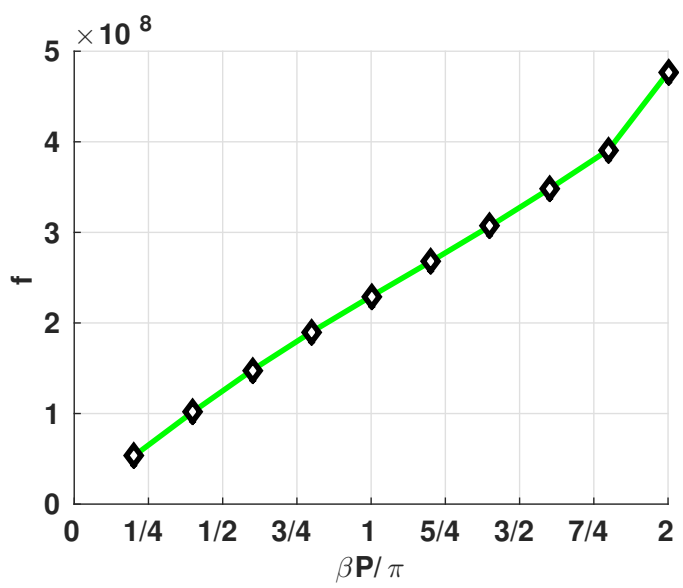

(a)

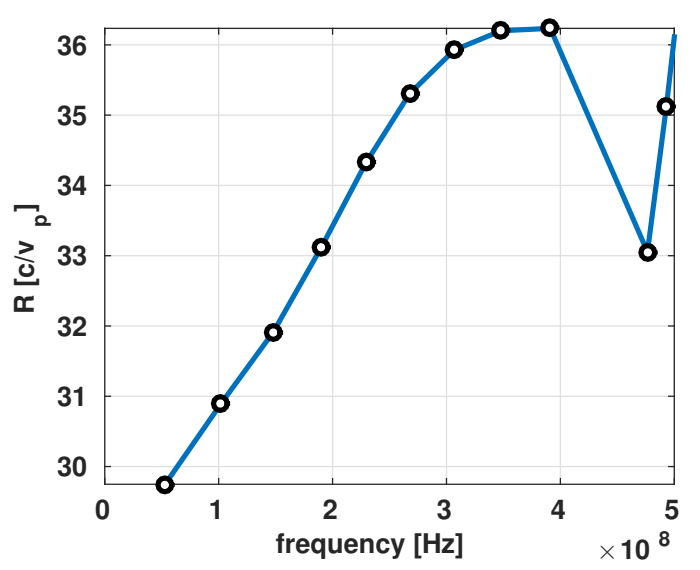

(b)

Figure 7.1: (a)Dispersion and (b) the retardation vs. frequency of SW3 as calculated from the VSim model.

Another thing to note about Fig. $7.1(\mathrm{a})$ is that from $\beta P / \pi=7 / 4$ to 2 , the slope changes which corresponds to the cutoff mode. See Sec. 6.2.2.2 for the explanation 
on cutoff. This cutoff region corresponds to a frequency of $400 \mathrm{MHz}$ and causes the large change in retardation shown in Fig. 7.1(b). The modes after $400 \mathrm{MHz}$ are still correct and exist, but the equation used to find the wavelength is incorrect for these modes, so retardations after $400 \mathrm{MHz}$ are not correct. These data points are included in Fig. 7.1 to make cutoff apparent and obvious.

\subsection{Electron Optics in Vsim}

An electron beam optics study was required in the Vsim simulation in order to match the injected beam experiment. The actual beam optic configuration is not actually modeled in the simulation but is an approximation. This approximation is used to increase the simplicity of the simulation model and decrease the simulation time. The injected beam cathode placement and optics affected the gain of the simulated CFA greatly, so a detailed description is given here. Fig. 7.2 shows the placement of the beam electrode and placement of the cathode relative to the SW circuit and sole electrode in VSim. Originally, the beam electrode was placed there to overcome the space charge limit of the cathode in order to be able to inject currents $>150 \mathrm{~mA}$. The electrode helped with that issue but was also found to affect the gain at lower beam currents. Two studies were performed on the effect on gain of the beam electrode potential and the cathode placement, and the results are outlined in the following sections. 


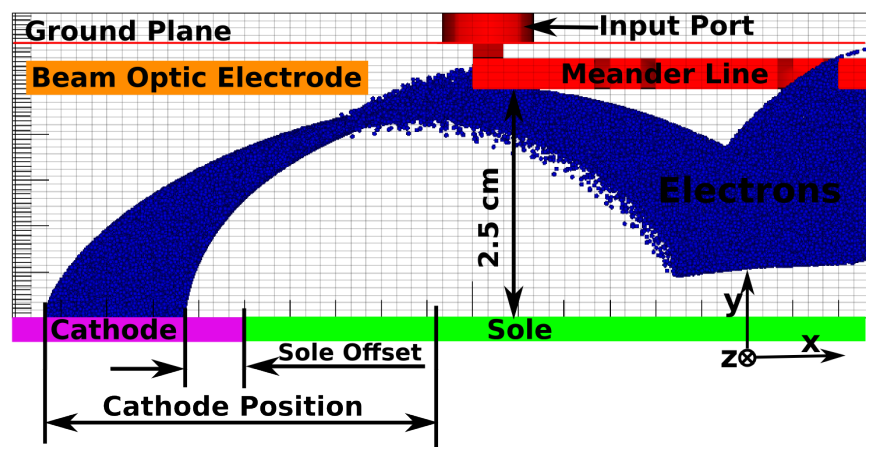

Figure 7.2: Vsim model showing the dummy electrode used to control the beam injection in the NU CFA and the cathode placement dimensions.

\subsubsection{Cathode Placement}

The placement of the injected beam cathode affects the gain of the device. A study was performed to determine the optimum position of the cathode in order to maximize the gain. The cathode area and placement in the $z$-direction is kept constant, while the cathode is moved in the $x$-direction. For the following example $I_{\text {beam }}=150 \mathrm{~mA}$, $B=5.4 \mathrm{mT}$, and $V_{a s}=1250 \mathrm{~V}$. Fig 7.3 shows the gain and the measured currents of this study. A description on the measured currents is given in Sec. 5.3.2.4. The gain shows a $0.5 \mathrm{~dB}$ difference from the cathode placement with the maximum gain at $4 \mathrm{~cm}$. 


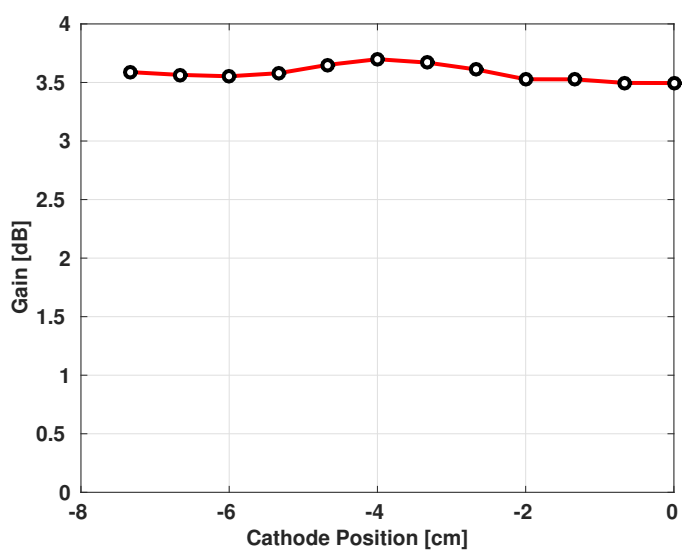

(a)

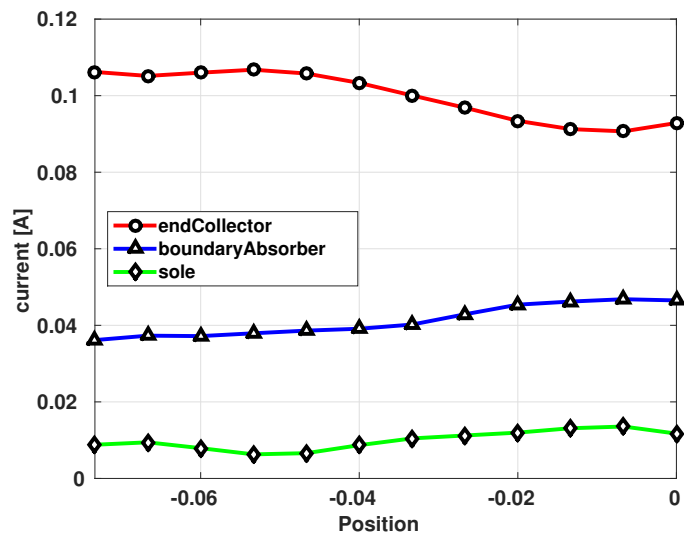

(b)

Figure 7.3: (a) Gain and (b) the measured currents on various electrodes vs. the position of the cathode for $I_{\text {beam }}=150 \mathrm{~mA}, B=5.2 \mathrm{mT}$, and $V_{a s}=$ $1250 \mathrm{~V}$. The Position at $\mathbf{0} \mathbf{~} \mathbf{m}$ corresponds to directly underneath the first period of the slow wave circuit.

To explain the gain variation with the cathode placement, the currents are shown in Fig. 7.3(b), and some electron trajectories from VSim are shown in Fig. 7.4. The currents show a $10 \mathrm{~mA}$ shift from going from the slow wave circuit to the end collector as the cathode position moves from 0 to $4 \mathrm{~cm}$. This current shift may have something to do with the gain, but the difference is not thought to be the main reason. After studying many of these current plots in different situations, this change in current was determined to be minor. Losing current to the SW circuit towards the end of the tube is not so detrimental to gain because those electrons have already given up some of their energy. However, current lost at the beginning of the tube is a serious problem for gain. The gain of the device is affected by a delicate balance of the closeness of the beam to the slow wave circuit but not too close as to lose all the beam at the slow wave circuit input. It is common to lose $\sim 50 \mathrm{~mA}$ out of $150 \mathrm{~mA}$ to the $\mathrm{SW}$ circuit, and this is often coincides with the optimum gain. 


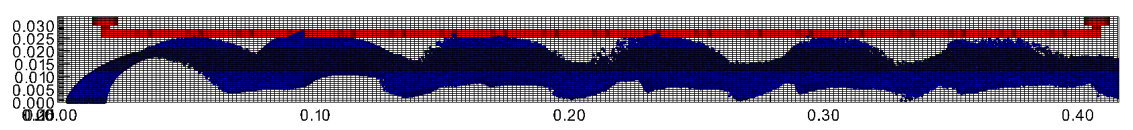

(a)

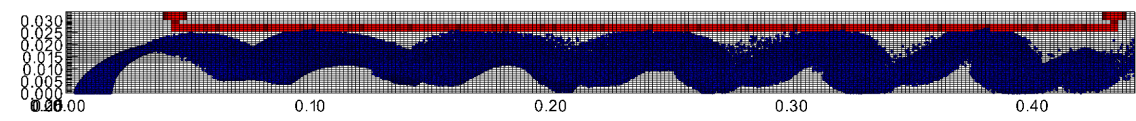

(b)

Figure 7.4: Electron trajectories corresponding to (a) $1.3 \mathrm{~cm}$ cathode offset placement and (b) $4.0 \mathrm{~cm}$ cathode offset placement. Electrons are shown in blue and the slow wave circuit is red.

The maximum gain is achieved by having the first close encounter of the electrons happen at the circuit input and by maximizing the electron beam close proximity to the SW circuit. Fig. 7.4(b) shows the electron trajectories for the optimum cathode placement. The first peak in the cycloid trajectory happens just at the input of the circuit; whereas in Fig. 7.4(a), which shows a worse cathode placement, the peak occurs a few centimeters past the input. The optimum placement increases the gain in the first part of the SW circuit and allows for more electron coupling all the way down the tube. Also, Fig. 7.4(a) shows only 6 cycloids along the circuit, and Fig. 7.4(b) shows seven. During each cycloid, the electron beam travels close to the SW circuit. The higher gain observed is also attributed to increasing the number of close encounters with the circuit.

The sole offset distance is also shown to affect gain, which is the location of the cathode/sole boundary relative to the electron emission location. The electric field at the boundary is rather large due to the close proximity of the electrodes to each other, and the closer the boundary is to the electron emission point the more effect it has on the emission. The larger the potential difference between the sole and the 
cathode electrodes, and the closer the boundary is to the electron beam emission point, typically reduces the space charge current limit, and alters the electron beam trajectory. The sole placement is found to have a significant effect on gain. A study on the sole placement is not shown here for brevity. Even though this parameter is not optimized for the simulations shown here, relative comparisons can still be made.

\subsubsection{Beam Electrode Potential}

The potential of the beam electrode, $V_{b e}$, also affects the gain of the device. The dummy electrode was originally placed at its location in order to overcome space charge current limits when injecting high beam currents and as an approximation to the actual experimental electrode, but it was found to affect the gain of the device at lower currents. Full simulations were performed for various beam electrode voltages. Fig. 7.5(a) shows the gain vs. the beam electrode potential; and Fig. 7.5(b) shows the currents on the end collector, sole, cathode, and the boundaries. The boundary absorber includes all the boundaries excluded from the other defined electrodes, but mainly all of the boundary absorber current is collected on the meander line itself. 


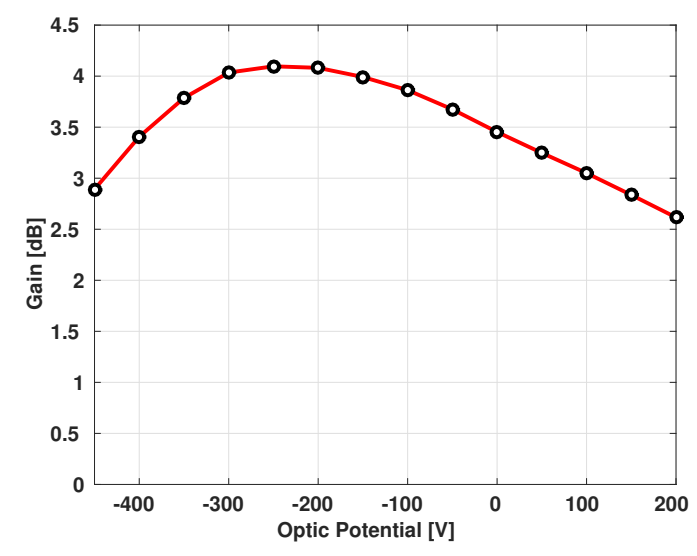

(a)

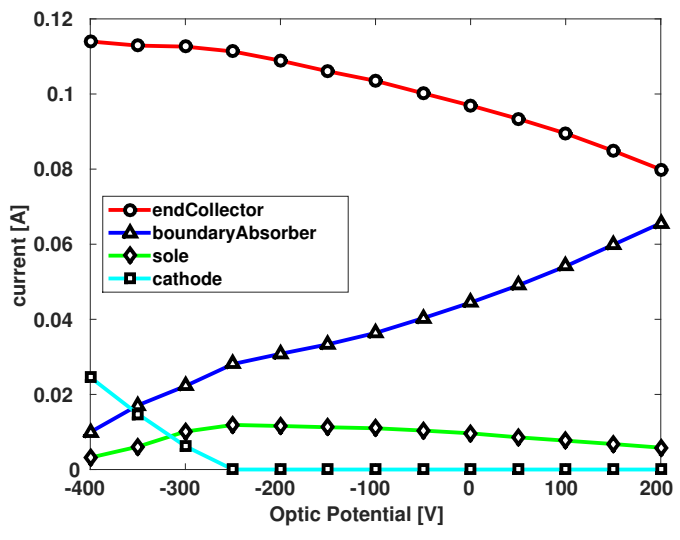

(b)

Figure 7.5: (a) Gain and (b) the measured currents on various electrodes vs. the potential of the beam electrode for $I_{\text {beam }}=150 \mathrm{~mA}, B=5.2 \mathrm{mT}$, and $V_{a s}=1250 \mathrm{~V}$. The Position at $\mathbf{0} \mathbf{~} \mathbf{m}$ corresponds to directly underneath the first period of the slow wave circuit.

Fig. 7.5 shows an optimum beam electrode potential at around -300 to $-200 \mathrm{~V}$. There are two things that determine the optimum beam electrode potential: the space charge limit and the beam proximity to the slow wave circuit in its first pass. The collected currents vs. beam electrode potential shown in Fig. 7.5(b) shows this effect well. As the beam electrode potential is decreased from $200 \mathrm{~V}$, the beam is pushed farther away from the circuit, allowing less current to be collected at the beginning of the slow wave circuit and allowing more current to pass down the tube and interact with the RF wave on the circuit. At $-300 \mathrm{~V}$ two things start to happen which cause a decrease in gain. The beam electrode potential is so low that space charge begins to limit the current out of the cathode, and the beam that is emitted moves farther away from the circuit.

This study has shown that the electron beam optics can be used to increase gain. In this case, only one beam electrode is used above the injection point, and the gain 
was improved greatly. Possibly two or more beam electrodes could improve the gain more. Alternate electron beam optic configurations are not tested here, but it is likely that more gain can be achieved with more complex configurations. Also, the experimental beam optic configuration shown in Fig. 2.30 could improve the gain as well. This case was not studied in order to minimize the complexity of the simulation model as explained earlier.

\subsubsection{Cathode to Sole and Anode to Sole Voltage Study}

The cathode to sole voltage, $V_{c s}$, and the anode to sole voltage, $V_{a s}$, also affect the gain of the device. The previous two sections only optimized the cathode placement and the beam electrode voltage while keeping $V_{a s}$ and $V_{c s}$ constant as to be consistent with the NU experimental data. In order to maximize the gain of the device with respect to all parameters, optimization with respect to $V_{a s}$ and $V_{c s}$ is studied here. Also, as is discussed in a later section, the distributed cathode required a change to $V_{a s}$ which further motivates a study on these parameters.

Changing $V_{a s}$ and $V_{c s}$ has a few effects on the beam trajectory. By increasing $V_{a s}$ alone, the $\mathbf{E} \times \mathbf{B}$ velocity and cycloid radius increases. To keep synchronous with the RF wave on the circuit, the magnetic field must be adjusted. For this study, the magnetic field is changed with anode to sole voltage as to keep synchronous with the RF field on the circuit. Keeping the retardation constant, increasing $V_{a s}$ and $B$ decreases the cycloid radius, which moves the beam farther away from the circuit. Changing the cathode to sole voltage, $V_{c s}$, changes only the height at which the electron beam cycloids in the interaction region. Increasing $V_{c s}$ moves the beam closer to the circuit. Fig. 7.6 shows the electron trajectories with $V_{a s}=1550 \mathrm{~V}$ and different $V_{c s}$ values. 


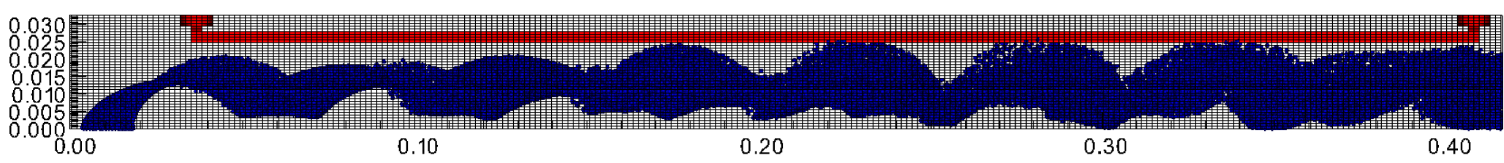

(a)

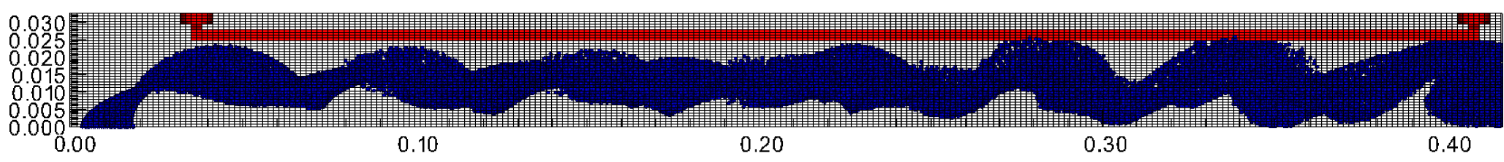

(b)

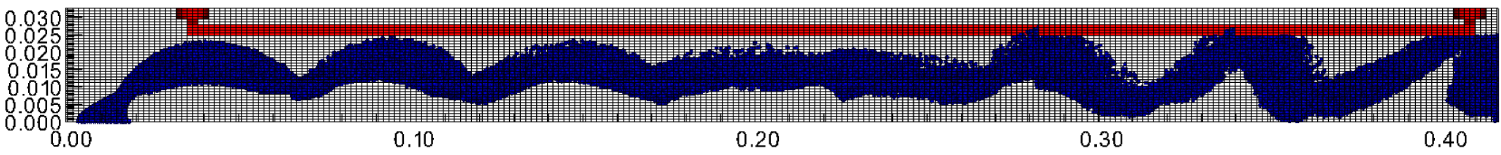

(c)

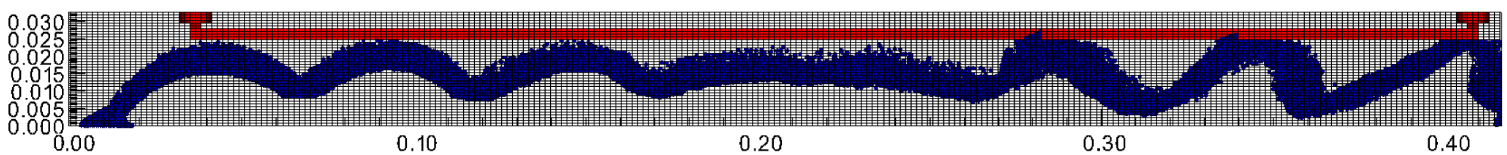

(d)

Figure 7.6: Electron trajectories for $V_{A S}=1550 \mathrm{~V}$, and different values of $V_{C S}$, (a) $200 \mathrm{~V}$, (b) $300 \mathrm{~V}$, (c) $400 \mathrm{~V}$, and (d) $500 \mathrm{~V}$.

As $V_{c s}$ is increased, the trajectories move closer to the circuit. Another observation which is not apparent from Fig. 7.6 is that as $V_{c s}$ increases, more current is lost at the cathode due to space charge limiting. This is because of the electric fields near the emission point, created by the cathode/sole boundary, causes a decrease in the space charge limit on emission current.

For this study, $V_{c s}$ was swept for various values of $V_{a s}$ to observe the effect on gain. Fig. 7.7(a) shows the relationship between gain and $V_{c s}$, and Fig. 7.7(b) shows the relationship between gain and $V_{a s}$. Both of these plots show the same data but from different perspectives. Fig. 7.7(a) shows the effect of changing the electron beam height. Fig. 7.7(b) better shows the effect of changing the cycloid radius. 


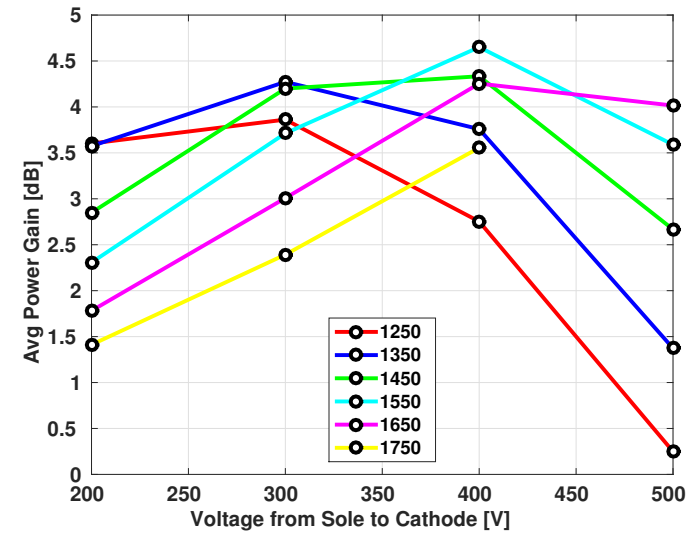

(a)

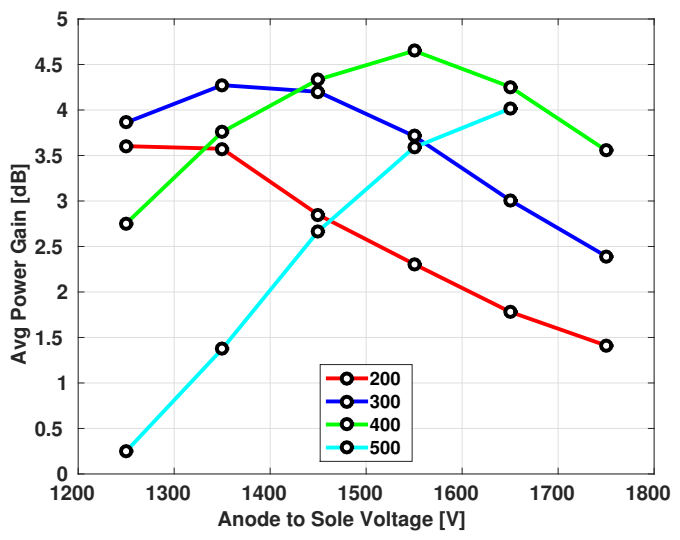

(b)

Figure 7.7: (a) Gain vs. $V_{c s}$ for various $V_{a s}$ and (b) Gain vs. $V_{a s}$ for various $V_{c s}$. The retardation is held constant by adjusting the magnetic field to keep the $V / B$ ratio constant.

The distance of the electron beam from the circuit limits gain when sweeping $V_{c s}$. When the beam is far from the circuit, the interaction impedance drops, lowering the gain. When the beam is too close to the circuit, current is lost to the circuit before giving up energy to the RF wave, lowering the gain. Note that each plot in Fig. 7.7(a) has an optimum $V_{c s}$ where the beam is close enough to interact without losing too much current at the slow wave circuit input. Note that as $V_{a s}$ is increased, the optimum $V_{c s}$ is also increased. This is because the cycloid radius decreases as $V_{a s}$ increases, moving the beam farther from the circuit, and increasing $V_{c s}$ moves the beam closer to the circuit again.

The distance of the electron beam from the circuit also limits gain when sweeping $V_{a s}$. Fig. 7.7(b) shows the relationship between gain and $V_{a s}$ for various values of $V_{c s}$. As $V_{a s}$ is increased, the cycloid radius decreases, which moves the beam farther from the circuit. For $V_{c s}=200-500 \mathrm{~V}$, the gain increases at first as the lost current to the circuit decreases. As the cycloid radius decreases farther as $V_{a s}$ increases, the beam 
moves too far from the circuit and gain decreases. Note that as $V_{c s}$ is increased, the optimum $V_{a s}$ is increased.

Each optimum $V_{c s}, V_{a s}$ combination does not have equal gain. The optimum combination for gain was found to be with $V_{c s}=400 \mathrm{~V}$ and $V_{a s}=1550 \mathrm{~V}$. The reason this is the best combination is that it has the best beam injection optics. Note that the optimum condition has less to do with the beam power and more with the beam injection optics. The injected beam power increases as $V_{a s}$ increases, but because the beam moves farther away, there is less interaction with the beam. To counteract this, $V_{c s}$ is increased, which actually lowers the beam power. The beam power ranges from $150 \mathrm{~W}$ to $180 \mathrm{~W}$ at the optimum combination for each $V_{a s}$ used. The $V_{c s}=400 \mathrm{~V}$ and $V_{a s}=1550 \mathrm{~V}$ combination does in fact have the highest beam power of the cases tested, but it is not thought to be the reason for the increase in gain. In fact, according to Pierce theory, summarized in Sec. 2.5, raising the voltage drop of the beam alone should decrease the gain per length of the device. The moderate changes in gain from each optimum value seems to indicate this effect, and the differences are attributed to beam injection optics.

\subsection{Injected Beam Characterization}

To confirm the simulation results, two sweeps were performed in Vsim: beam current sweep and a bandwidth sweep. Experimental results of these sweeps were provided in $[14,15]$ and are shown in Figs. 2.32 and 2.31. The optimum magnetic field for the maximum simulated gain differed by $3 \mathrm{mT}$ from the experiment. The simulation uses the optimum value for $B$ to compared with experiment, and the justification for this is discussed later when the general characterization and optimization of $B$ is 
provided.

\subsubsection{Optimum Magnetic Field}

To match the experiment as closely as possible, the potential between the anode and the sole is kept the same as the experiment, $V_{a s}=1250 \mathrm{~V}$. The retardation of SW3 in Vsim is slightly off from the retardation found in the experiment, so it is expected that the optimum gain will be at a different magnetic field. A magnetic field sweep is shown here to find the maximum gain and is shown in Fig. 7.8 with the corresponding currents. The optimum magnetic field is at $5.4 \mathrm{mT}$, which corresponds to an $\mathbf{E} \times \mathbf{B}$ velocity 31.9 times slower than the speed of light. This optimum velocity of the beam matches with the phase velocity of the RF wave in the circuit at $150 \mathrm{MHz}$ as shown in Fig. 7.1(b). As the magnetic field changes, the $\mathbf{E} \times \mathbf{B}$ velocity of the beam deviates from the RF phase velocity and the gain decreases. The optimum simulated magnetic field differs from the experimental optimum by $1-3 \mathrm{mT}$, which is due to small geometric differences from the simulated to experimental model.

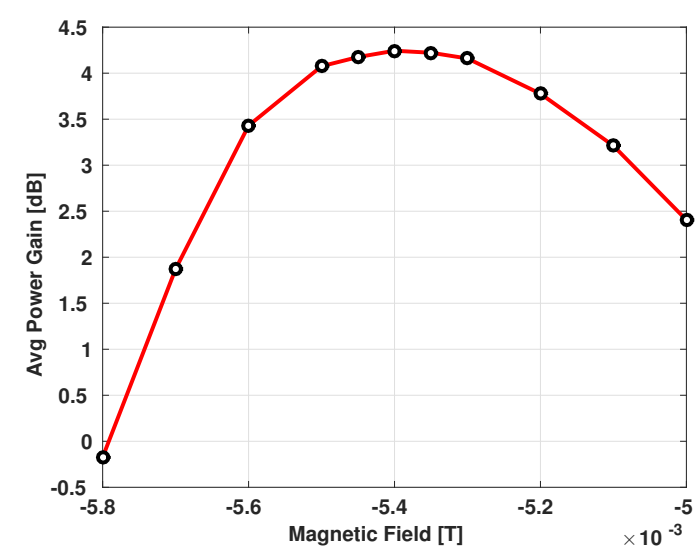

(a)

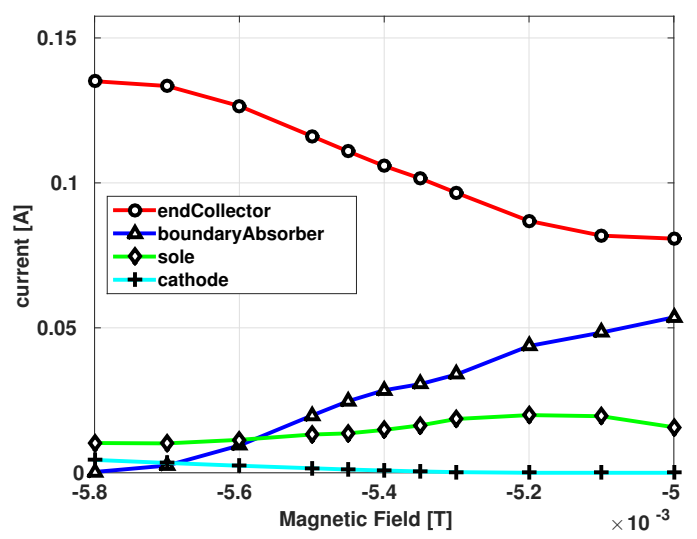

(b)

Figure 7.8: (a) Gain vs. Magnetic Field for $I_{b e a m}=150 \mathrm{~mA}, V_{a s}=1250 \mathrm{~V}$, $V_{b o}=-200 \mathrm{~V}$ and (b) the corresponding currents from VSim. 
Also note that the current to the slow wave circuit, shown in Fig. 7.8(b), increases as the magnetic field decreases. This decrease is due to the cycloid radius increase as the magnetic field decreases, which brings the electron beam closer to the circuit. This lost current does affect the gain of the device, but it is not the reason for the decrease in gain at lower magnetic fields. Magnetic field sweep simulations, not shown here, were performed with a higher $V_{a s}$ to reduce the cycloid radius so that little to no current collects on the slow wave circuit, and all showed the same optimum $\mathbf{E} \times \mathbf{B}$ velocity.

\subsubsection{Beam Current Sweep}

This simulation sweep increases the beam current while keeping $V_{\text {sole }}=-1250 \mathrm{~V}$, $V_{\text {cathode }}=-1050 \mathrm{~V}$, and $B=5.4 \mathrm{mT}$. The beam electrode is varied at each beam current to help overcome the space charge limit and inject the current properly in order to maximize gain. Fig. 7.9 shows the experimental $[14,15]$ and simulated gain vs. beam current and the simulated collected currents of the sweep. 


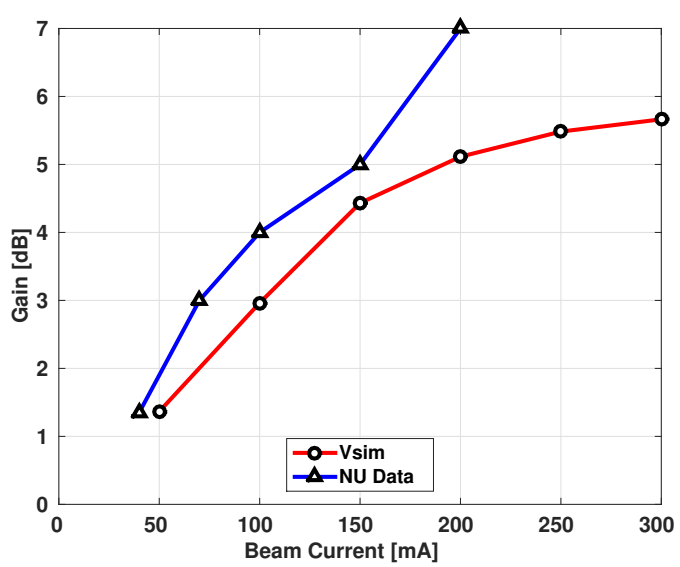

(a)

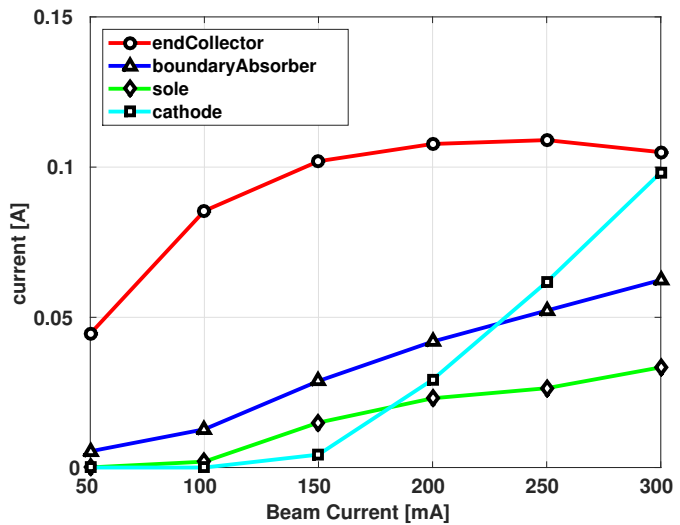

(b)

Figure 7.9: (a) Gain vs. injected beam current and comparison to experimental data found in Browning et al. [14, 15] and (b) the corresponding currents from VSim.

The first thing to note is that as the current is increased, the gain increases. This is as expected because there is more power available for gain allowing the RF wave to extract more power. The next thing to note is that the experimental and simulated data match relatively well except for two things. One is that the simulated gain is lower by about $1 \mathrm{~dB}$ from the experimental data, and the other is that the simulated gain rolls off at about $200 \mathrm{~mA}$; whereas the experimental gain does not.

It is speculated that the lower gain is due to differences in the electron beam optics. The beam electrode study in Sec. 7.2 showed that the gain is very sensitive to the beam injection into the device, and this sensitivity was apparent by only changing the cathode placement and the one beam electrode potential. The experimental configuration had more sophisticated beam electrode optics, and this difference could be one reason for the discrepancy. Another reason could be the difference in the slow wave meander lines. The simulated design uses a rectangular cross section for the line, and the experimental configuration uses a copper tube. This circular cross-section 
is thought to allow more of the RF wave to travel outside the dielectric and, thus, have a higher interaction impedance. A study on the comparison of the interaction impedance between rectangular and circular cross-sections is not performed here.

The reason the gain rolls over at $200 \mathrm{~mA}$ is also due to non-optimum electron beam optics. The potential on the beam electrode is optimized for each data point on the curve. For beams with low current $(<150 \mathrm{~mA})$, the beam electrode optimizes the trajectory well without suppressing any current due to the space charge limit. For beams $>150 \mathrm{~mA}$, the beam electrode needs to balance between the optimum trajectory and overcoming the space charge limit. In order to overcome the space charge limit for higher currents, the beam electrode potential needs to be larger, and the beam tends to strike the SW circuit right away, and less gain is observed. Fig. 7.9(b) shows the collected currents. Note that at $200 \mathrm{~mA}$ and above, more and more current is collected at the cathode. This is suppressed current due to space charge. Any additional current is just suppressed, and no improvements to gain are observed.

In order to optimize both the trajectory and injected current, more beam electrodes are needed. Regardless, the curve trends match relatively well, and it is thought that with proper beam control the gain will increase and the rollover will be prevented to better match the experimental results. Adding proper beam optics to the simulation would increase the simulation size and time and require extensive model development time, so such a study was not performed. The results were deemed close enough to the experiment to move on to the next phases of the project.

Another correlation that is not immediately apparent from the gain and current plots is that the efficiency goes up with higher currents. Fig. 7.10 shows the efficiency vs. the beam current for both the Vsim and the Northeastern data. the first thing to note is that the efficiency is quite low $(<16 \%)$. This low efficiency is mainly due 
to the short length $\left(6 \lambda_{s w}\right)$ compared to more efficient CFAs with $\sim 15 \lambda_{s w}$. The efficiency of the simulated data is, of course, lower than the experiment because of the lower gain achieved. The trend that the efficiency increases with beam current is the same. The experimental data shows a small decrease at $I_{\text {beam }}=150 \mathrm{~mA}$, but the next data point at $I_{\text {beam }}=200 \mathrm{~mA}$ shows an increase again. The explanation of this outliers is unknown; however it is thought to be due to an unoptimized setup, and the trend that efficiency increases with current is still considered to be correct. The simulated data shows the efficiency leveling out at $I_{\text {beam }}=200 \mathrm{~mA}$ which is due to the current lost to the cathode from space charge. With proper beam optics, the efficiency should continue to increase. The increase in efficiency with respect to beam current is consistent with Pierce theory so long as current is not suppressed.

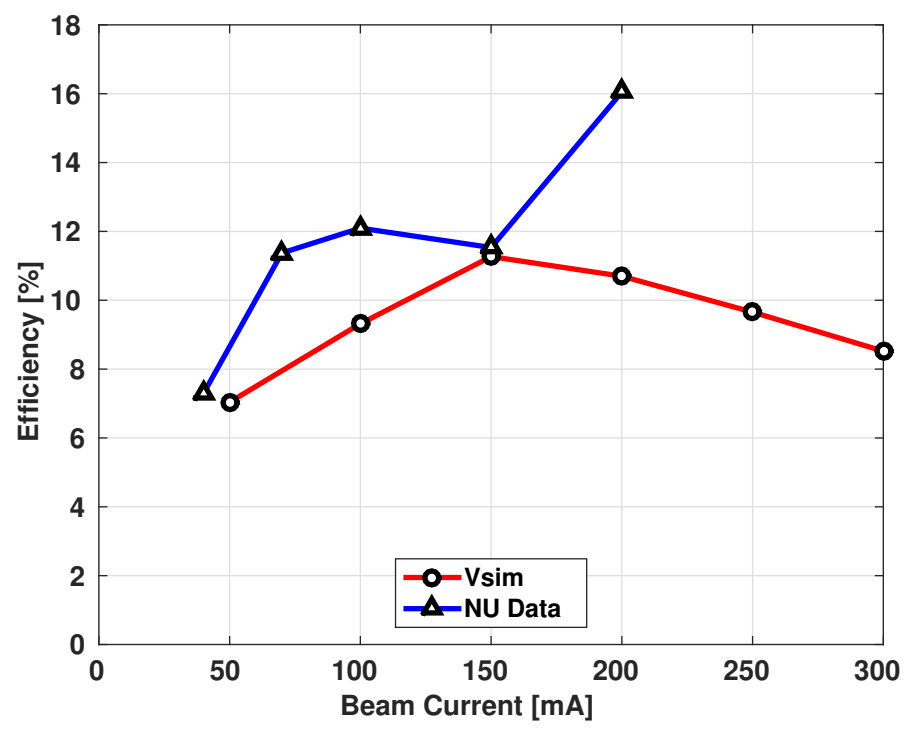

Figure 7.10: Efficiency vs. injected beam current and comparison to experimental data found in Browning et al. $[14,15]$ 


\subsubsection{Bandwidth Sweep}

This study looks at the frequency variation while keeping $V_{\text {sole }}=-1250 \mathrm{~V}, V_{\text {cathode }}=$ $-1050 \mathrm{~V}, I_{\text {beam }}=150 \mathrm{~mA}, V_{b e}=-200$ Vand $B=5.4 \mathrm{mT}$. Fig. 7.11 shows the gain vs. frequency plot comparison with experiment and the corresponding currents. There are a few things that will limit the bandwidth in this circuit, but the main limiter is the dispersion of the circuit. Because of the dispersion, the RF phase velocity changes as the frequency is varied. For a constant $\mathbf{E} \times \mathbf{B}$ velocity of the beam, maximum gain occurs when the retardation of the circuit matches that of the beam velocity. The gain decreases when there is a $\mathbf{E} \times \mathbf{B}$ and phase velocity mismatch.

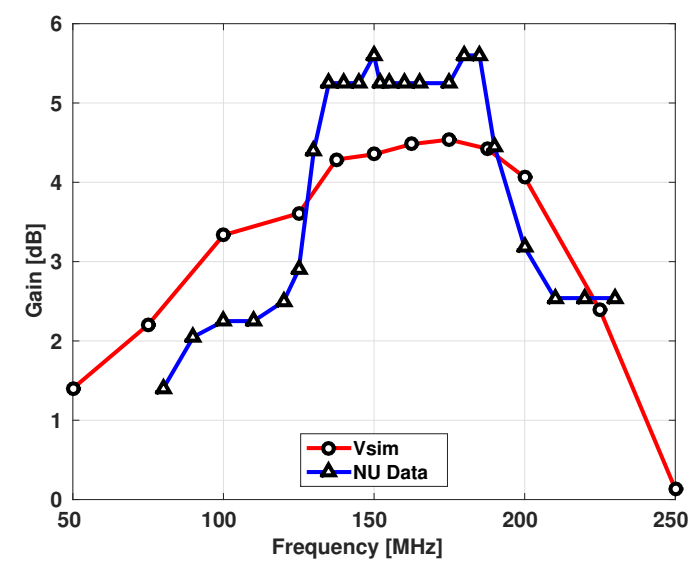

(a)

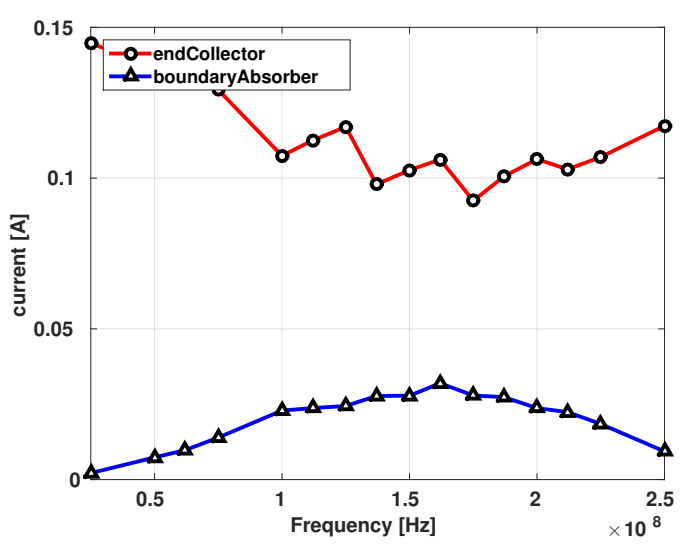

(b)

Figure 7.11: (a) Gain vs. Frequency and comparison to experimental data found in Browning et al. [14] and (b) the corresponding currents

There are a few similarities and differences between simulation and experiment. The maximum gain of the simulation is lower by about $1 \mathrm{~dB}$, but this difference is thought to be due to imperfect beam optics in the simulation as discussed in Sec. 7.3.2. The frequency of maximum gain of the simulation matches well with that of the experiment. The shapes are different, and the bandwidth of the simulation is 
larger. The reason for the small experimental bandwidth and the sharp drop in gain is thought to be from another limitation on bandwidth with the experimental setup, including the RF connections, that are not currently modeled in the simulation. Note that gain in the experimental data does not fall directly to zero at 110 and $200 \mathrm{MHz}$; it goes to $\approx 2 \mathrm{~dB}$. This is rather unusual for bandwidth tests. Normally, it should fall directly to zero gain. The simulated data is smooth and falls steeply to zero gain. For this reason and the fact that the centers of the experimental and simulated bandwidth match up, the simulation is thought to be correct. The reason the experimental data has this rather unusual behavior and has a smaller bandwidth could be due to the connectors, the copper tube meander line, the dielectric mounting screws, or imperfections in the circuit. The ports in the simulation are rather idealized, so any bandwidth limitations of the interconnects would not be apparent in the simulation.

\subsection{Injected Beam Studies}

To get a good comparison between a distributed cathode implementation and the injected beam, a few studies were performed to characterize the injected beam configuration. One study attempts to show the relationship between beam and RF power. Another study determines the relationship between gain and the length of the slow wave circuit.

For many of the studies, instead of using $V_{a s}=1250 \mathrm{~V}, V_{a s}=1550 \mathrm{~V}$ was used because the distributed cathode is better optimized at this voltage and because testing the injected beam configuration at this same voltage allows for a direct comparison of the different methods. A detailed justification for this change in anode to sole voltage is explained in Sec. 7.5.3.2. The injected beam configuration with $V_{a s}=1550 \mathrm{~V}$ is 
optimized with respect to cathode placement, the cathode to sole voltage, and the beam electrode voltage per the same method described in Sec. 7.2. The optimum parameters were found to be $V_{c s}=400 \mathrm{~V}$ and $V_{b o}=100 \mathrm{~V}$.

\subsubsection{Resolution Study}

To determine the minimum requirements of the grid in order to minimize simulation time, a study on the grid size was performed. The geometric constraint on the size of the grid is determined by the smallest geometric feature along the corresponding axis. In the VSim model, the smallest geometric feature is the slow wave circuit line width in the $x$ - and $z$-directions and the dielectric height in the $y$-direction. Reference Figures 5.4 and 5.5 in Sec. 5.3.1 for the geometric grid overlay diagrams. The higher the resolution, the more accurate the solution, and the minimum requirement for grid size is determined when any increase in the resolution to the grid yields little to no difference in simulation result.

To test the grid integrity, the number of cells per line width was increased from $N_{L W}=2$ to $N_{L W}=3$, and the number of cells per dielectric was changed from $N_{d}=2$ to $N_{d}=3$. This variation, since the grid was coarse to begin with, changed the dimensions of the CFA model slightly. As discussed in Sec. 5.3.1, since each geometric feature is not divisible by the cell size, each feature is slightly adjusted to align with the grid. The circuit pitch actually stays the same with this resolution change, which keeps the RF phase velocity consistent between the models, but the anode-to-sole distance changes from $H_{a s}=2.46 \mathrm{~cm}$ to $H_{a s}=2.49 \mathrm{~cm}$. This seems like

a small change in distance, but this change decreases the static electric field enough in the interaction region to require a $1 \mathrm{mT}$ decrease to the optimum magnetic field to match the beam velocity to the unchanged $R F$ wave phase velocity. The electron 
beam cycloid radius is increased, which requires a new optimization of the beam electrode potential. Also, the decrease in the static electric field increases the effect of space charge on the emission current, which also requires an optimization of the beam electrode potential.

The results of the resolution study are not presented here because the of the fact that the geometry is different between the two models. The gain using the two different grids were comparable, but required a different magnetic field and beam electrode potential. The goal of the resolution study is to confirm that no simulation artifacts are created at the low resolution, and even though the geometry is different, the physics are maintained in the lower resolution.

\subsubsection{RF Power and Beam Power Gain Study}

This study attempts to find the effect on gain and noise from the relationship between the beam power and RF power for the injected beam case. One aspect is finding the value at which gain is observed for the lowest $R F$ power on the circuit. The ratio between the beam power and the RF power cannot be too high because the beam will swamp the signal on the circuit. Measuring the output powers when using low RF input powers was found to be very difficult because of the noise. Many different methods to calculate gain were tested, and three different methods are described here. The method to calculate the signal-to-noise ratio is also presented here.

\subsubsection{Gain Calculation Method One: Average Power Method}

This method finds the gain by using the average output power over $N$ periods during steady state using the Poynting vector diagnostic divided by the known input power. The average must be averaged only during steady state and over an integer multiple 
of the period. The known input power is used because the input power measured by the Poynting vector diagnostic becomes corrupted by noise from the electron beam at lower powers. The formula for gain is shown in Eq. (7.1) where $p_{\text {out }}(\theta)$ is the instantaneous output power, $\theta=\omega t, \omega=2 \pi / T$, and $\theta_{\text {end }}$ is the angle at the end of the simulation.

$$
\text { Gain }=\frac{-\frac{1}{2 \pi N} \int_{\theta_{\text {end }}-2 \pi N}^{\theta_{\text {end }}} p_{\text {out }}(\theta) d \theta}{P_{\text {in }}}=\frac{P_{\text {out }}}{P_{\text {in }}}
$$

\subsubsection{Gain Calculation Method Two: Squared Voltage Ratio Method}

This method attempts to remove any DC noise from the power calculation. A weakness of method one is that any DC current on the circuit caused by the electron beam collecting on the circuit is included in the calculation. Eq. (7.2) shows the method where $V_{A C}$ is the RMS voltage of the AC coupled steady-state signal over $N$ periods. The ratio is squared to get the gain in terms of power.

$$
\text { Gain }=\left(\frac{V_{\text {ACout }}}{V_{\text {ACin }}}\right)^{2}
$$

\subsubsection{Gain Calculation Method Three: Power Spectral Density (PSD) Method}

This method attempts to obtain the power at the desired frequency. The weakness in the previous methods is that they do not account for the noise power. To obtain the power at the desired frequency, the power spectral density (PSD) is found using the steady-state voltage of $\mathrm{N}$ periods of both the input and the output. The gain is found by finding the ratio of the output power and the input power at the desired frequency $f_{0}$, shown in eq. (7.3). 


$$
\text { Gain }=\frac{P_{P S \text { Dout }}\left(f_{0}\right)}{P_{P S \text { Din }}\left(f_{0}\right)}\left[\frac{\mathrm{W}}{\mathrm{W}}\right]=P_{\text {PSDout }}\left(f_{0}\right)-P_{P S \text { in }}\left(f_{0}\right)[\mathrm{dB}]
$$

\subsubsection{Signal-To-Noise Ratio Calculation}

The SNR is calculated using the $\operatorname{snr}()$ function in MATLAB, which uses the FFT of the signal and takes the ratio between the power at the operating frequency and the power contained at all other frequencies. The DC component and one harmonic frequency is excluded in this ratio. The SNR values presented here are not an indication of absolute SNR and can only be used for relative comparisons. The simulation run time is not long enough, and the macroparticle size is too large to get an accurate reading of SNR, but because each of the simulations are run with similar parameters, relative comparisons can be made.

\subsubsection{Results and Discussion}

The RF input power was swept from $10^{-4}$ to $10 \mathrm{~W}$ using the optimum parameters for the injected beam $\left(V_{a s}=1550 \mathrm{~V}, V_{c s}=400 \mathrm{~V}, V_{b e}=100 \mathrm{~V}\right)$ with $I_{\text {beam }}=150 \mathrm{~mA}$. Fig. 7.12 shows the gain and corresponding efficiency for the three methods at different RF input powers. From $10^{-2}$ to $10 \mathrm{~W}$ the three methods show the same gain. From $10^{-4}$ to $10^{-2} \mathrm{~W}$ the three methods diverge. These differences will be discussed in more detail later. First, the behavior from $10^{-2}$ to $10 \mathrm{~W}$ is discussed. 


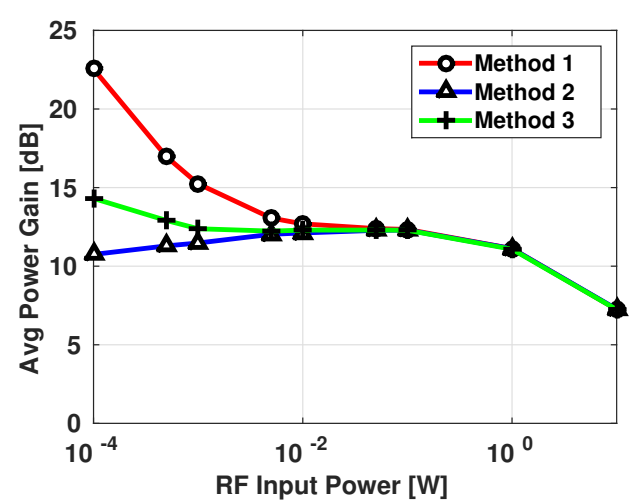

(a)

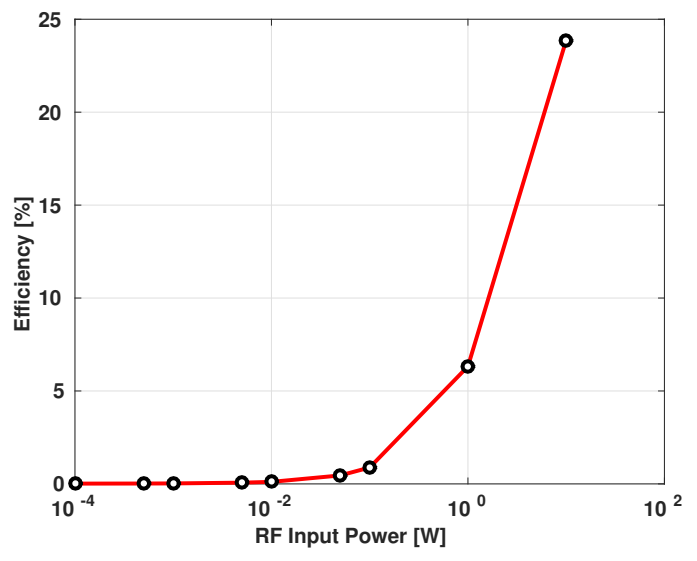

(b)

Figure 7.12: (a) Gain vs. RF power for $I_{\text {beam }}=150 \mathrm{~mA}$ and (b) the corresponding efficiency vs. RF input power.

The decrease in gain from $10^{-1}$ to $10 \mathrm{~W}$ is caused by the power in the beam being depleted before reaching the end of the CFA. The beam power is calculated by the voltage difference between the cathode to the anode times the current, $P_{\text {beam }}=$ $V_{\text {ca }} I_{\text {beam }}=172.5 \mathrm{~W}$. According to Pierce theory, the gain should be constant for all powers, in this case about $12 \mathrm{~dB}$. For the $10 \mathrm{~W}$ case, $12 \mathrm{~dB}$ makes the output power $168 \mathrm{~W}$. This would make the device almost $100 \%$ efficient, while it is actually closer to 20-30\%; so as the RF input power increases, the gain must decrease as the beam power limit is approached.

The power from $10^{-2}$ to $10^{-1} \mathrm{~W}$ stays constant, which is what Pierce theory predicts. The power is low enough compared to the electron beam power so that there is plenty of energy to extract without being limited by the beam power. Also, the RF input power is high enough compared to the beam so that the signal-to-noise (SNR) ratio is high for both the input and output signals. This allows for an accurate reading of power, using all calculation methods; whereas for RF input powers lower 
than $10^{-2} \mathrm{~W}$, it is difficult to determine the power because of the noise.

The deviation of the three methods for powers less than $10^{-2} \mathrm{~W}$ is due to the noise created by the electron beam, and each method responds differently to the noise. Figure 7.13 shows the SNR of the voltage signal for the different RF input powers.

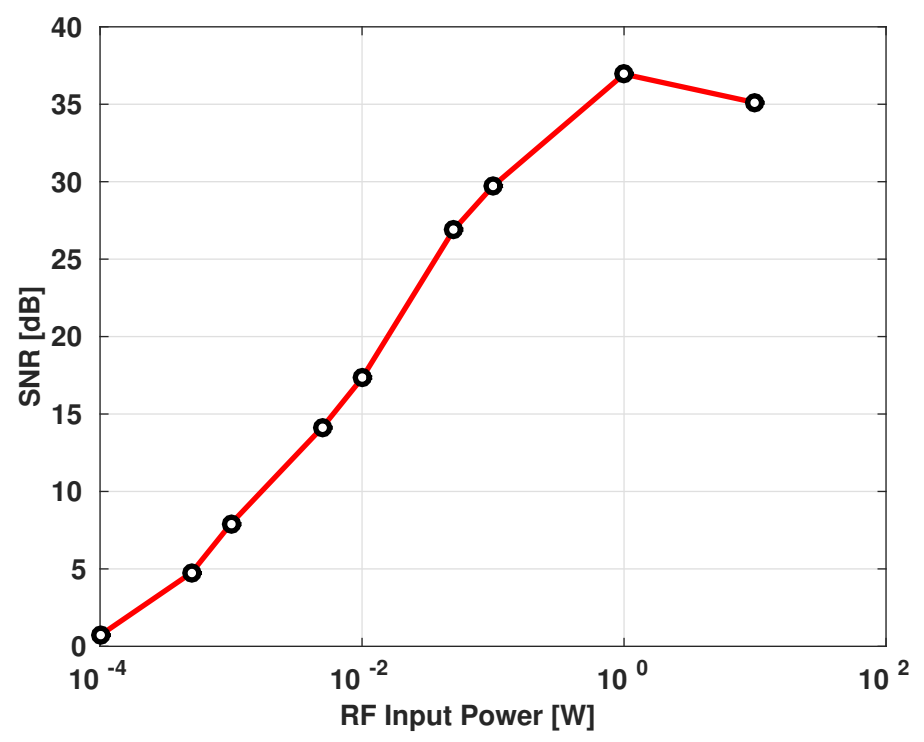

Figure 7.13: Signal-to-Noise ratio for different powers

Figure 7.13 shows that the SNR ratio increases as the RF input power increases. This is to be expected because the electron beam introduces a consistent noise to the signal, and as the RF input power is decreased, the electron beam noise becomes comparable to the signal, thus reducing the SNR. The SNR ratio increases by $10 \mathrm{~dB}$ per decade as the power increases. This indicates that the noise created by the beam remains relatively constant as the RF input power increases. The output power increases by $10 \mathrm{~dB}$ as the input power is increased by $10 \mathrm{~dB}$, which increases the SNR by $10 \mathrm{~dB}$ because the noise level created by the beam stays constant. The decrease in SNR at the RF input of $10 \mathrm{~W}$ is due to the output power decreasing from the limited 
power extractable by the beam. This phenomenon is explained in greater detail in Sec. 7.4.3. Because the output power does not increase past RF input powers of $1 \mathrm{~W}$, the SNR levels out.

Figure 7.14 shows the voltage signal for a RF input power of $10 \mathrm{~mW}$, where all methods match for calculating gain, and for $0.1 \mathrm{~mW}$, where all methods deviate. The first thing to observe is the large pulse on the output power at $t=60 \mathrm{~ns}$. This pulse is caused by the electron beam front as it passes by the output port. Thereafter, the amplified RF wave can be observed. The output signal in Fig. 7.14(a) is somewhat noisy, but the $150 \mathrm{MHz}$ component is easily observable. The input signal is also observable, but it has a DC component. This DC component is caused by the electron beam. The input and output signals in Fig. 7.14(b) are both very noisy. The $150 \mathrm{MHz}$ signal on the output is still visible, but difficult to interpret, and there is a DC offset. The $150 \mathrm{MHz}$ signal on the input is not visible anymore, and there is a $1 \mathrm{~V} \mathrm{DC}$ offset.

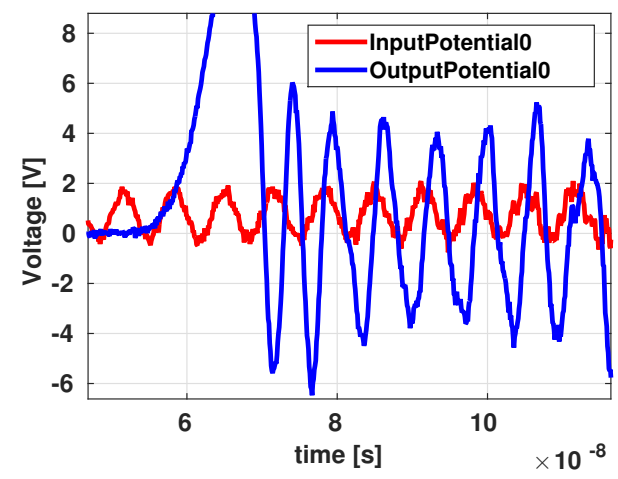

(a)

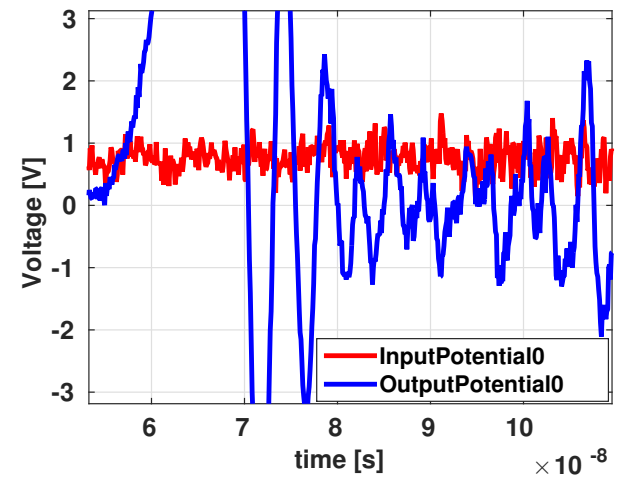

(b)

Figure 7.14: The input and output voltage with $I_{\text {beam }}=150 \mathrm{~mA}$ for (a) $P_{r f}=10 \mathrm{~mW}$ and (b) $P_{r f}=0.1 \mathrm{~mW}$

The noise in the signal is the cause of the power measurement method deviation. Method one, which measures the average power directly from the Poynting vector 
includes not only the power contained in the signal but also the noise from the beam. This causes the apparent measured power to increase, as the SNR increases, thus increasing the apparent gain. Method two removes any DC power component by determining the gain from the RMS average of the AC coupled signals. This method shows the gain decreasing as the RF input power is decreased. The problem with this method is that AC noise created from the electron beam is not filtered out. This difference affects both the input and output signals, but increases the power measured from the input more than the output because of the higher SNR. Method three attempts to remove both the $\mathrm{AC}$ and $\mathrm{DC}$ noise from the input and output by determining the power using the power spectral density. The power determined by this method, shown in Fig. 7.12, is increasing as the RF input power decreases in this case. In other cases it can also decrease, but it always measures a gain in between method one and two. Figure 7.15 shows the RF input power sweep for the same optimum parameters but for $I_{\text {beam }}=100 \mathrm{~mA}$. In this case the gain decreases as the RF input power is lowered, but it is still in between the gain determined by the other two methods. The power determined by method three is also affected by noise, but not consistently. The simulation is only run for $5 \mathrm{RF}$ periods (33ns) in steady state, and the power spectral density (PSD) for both the input and output is only taken for RF 5 periods. This yields a coarse PSD, and it is still susceptible to noise, especially on the input, and is the main cause of error. The error in this case is also more random where higher or lower powers than actual can be measured. 


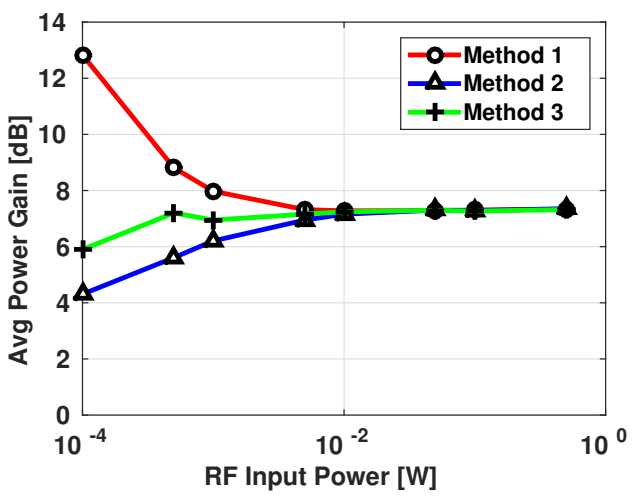

Figure 7.15: Gain vs. RF power for $I_{\text {beam }}=100 \mathrm{~mA}$

The three methods all determine different trends in gain for low powers, and all deviate from theory. Theory suggests that the gain will be the same for all powers as long as there is sufficient energy in the beam. The results seem to corroborate this theory but deviate as soon as the method to measure power becomes inaccurate. The best method here seems to be method three since it deviates from the constant gain line less than the other 2 methods. And with longer simulation times, the error from the method will improve. Regardless, the SNR ratio for these low powers is so low that it is doubtful any useful information in the signal can be used. The gain behavior at these low powers is not that important for this research. Therefore, the effect of longer simulation times on method three was not tested.

The minimum RF input power that can be used in a CFA is determined by the lowest usable SNR. All powers should have a relatively equal gain, as shown by simulation and Pierce theory, but the noise from the beam is the limiting factor. The beam noise is at a constant level, and the lowest RF input power is limited by the constant noise generated by the beam. 


\subsubsection{Gain vs. Circuit Length}

Two different studies were performed on the effect of the circuit length on gain. The first one studies the gain for five different slow wave circuit lengths. The second study is one simulation of a long circuit while monitoring the gain along the length of the circuit. The second study essentially shows the same result, but demonstrates the gain increase along the length of the circuit. It is also compared to other methods which indirectly measure the gain along the circuit.

The simulated gain along the length of the circuit is compared to Pierce theory [10], which is described in Sec. 2.5. The beam impedance is simply $Z_{B}=V_{c s} / I_{\text {beam }}=$ $10.3 \mathrm{k} \Omega$ and the wave number is $\beta_{0}=2 \pi / \lambda_{s w}=100.2 \mathrm{~m}^{-1}$. The interaction impedance is more difficult to calculate and requires a simulation to determine. To determine the interaction impedance, an RF input is applied to the circuit, and the model is simulated without particles. The RF amplitudes of the $x$ and $y$ electric field components are monitored at various distances from the circuit, and using Eq. (2.11) the interaction can be determined at these distances from the circuit. Pierce theory is derived using a laminar flow beam; the correct interaction impedance uses the one determined at the beam location. The NU CFA model uses a highly cycloidal beam, so determining the beam distance from the slow wave circuit is not straight-forward, which is why the interaction impedance is measured for many distances. The interaction impedance is roughly estimated to be $Z=7.4 \Omega$, which slightly lower than the impedance at the cycloid trajectory's closest encounter $(Z=9 \Omega)$. Using Eq. (2.15) the estimated gain is calculated. 


\subsubsection{Gain vs. Circuits of Different Lengths}

The longer the circuit, the longer the beam can couple power to the RF wave on the circuit and the greater the gain. To study this effect, the full CFA simulation was run at different SW circuit lengths for different RF powers. Figs. 7.16 and 7.17 show the gain and efficiency vs. length of $P_{r f}=1 \mathrm{~W}$ and $P_{r f}=10 \mathrm{~W}$, respectively. Also shown on these gain plots is the theoretical gain as predicted by Pierce theory. Both of these plots show the gain increases as the circuit length increases. The simulated gain follows Pierce theory for the shorter lengths and starts to level off at a constant gain. The lower RF input power has larger gain but lower efficiency.

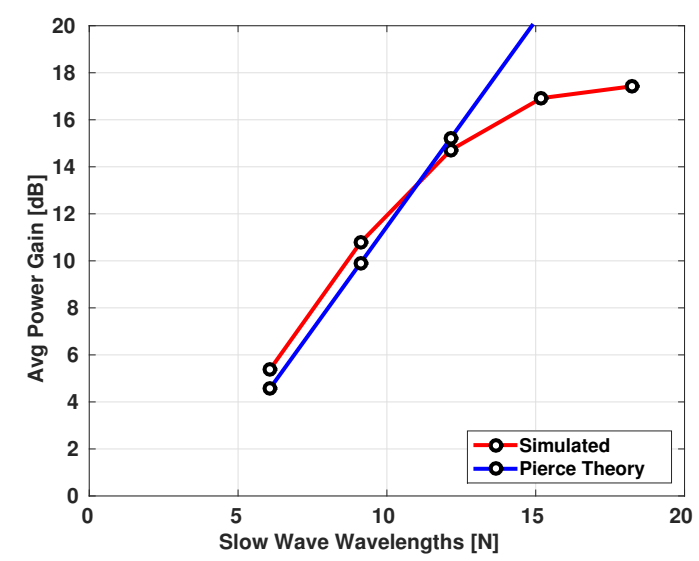

(a)

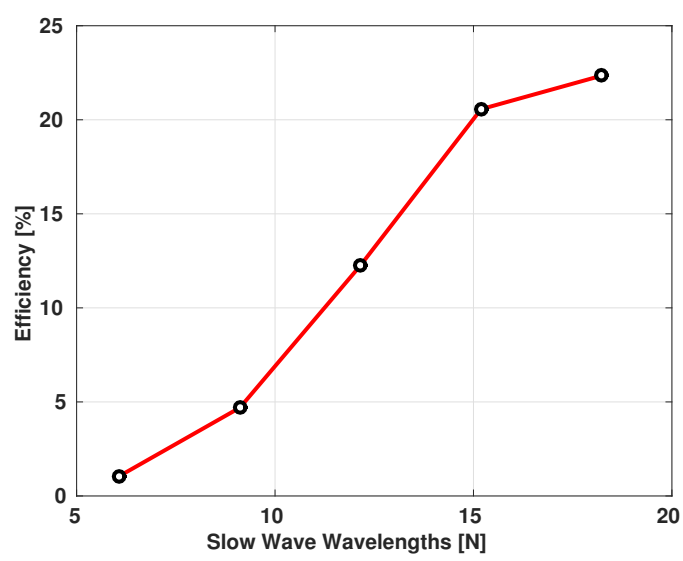

(b)

Figure 7.16: (a) The Gain vs. the SW circuit length with $P_{r f}=1 \mathrm{~W}$ and $I_{\text {beam }}=150 \mathrm{~mA}$ and (b) the corresponding simulated efficiency 


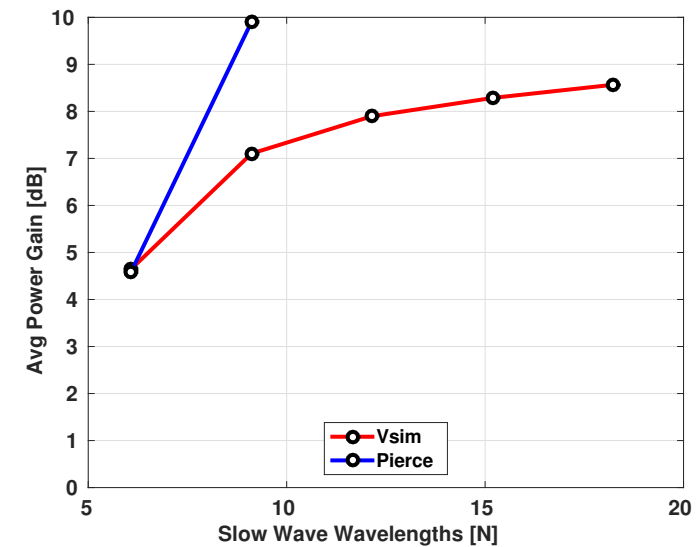

(a)

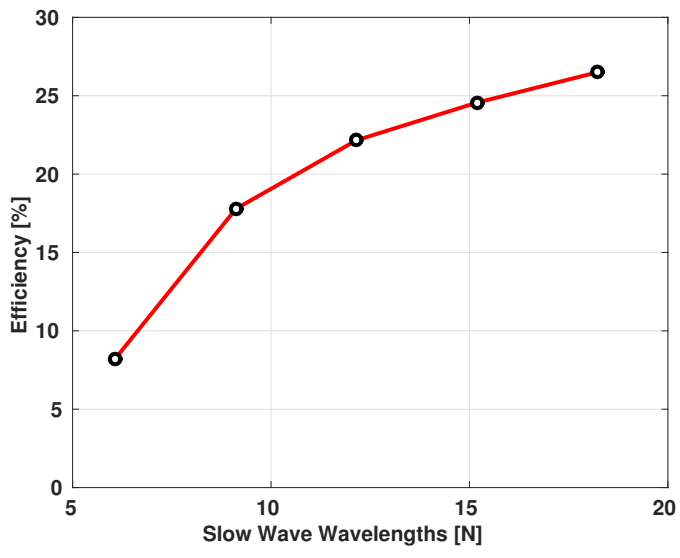

(b)

Figure 7.17: (a) The Gain vs. the SW circuit length with $P_{r f}=10 \mathrm{~W}$ and $I_{\text {beam }}=150 \mathrm{~mA}$ and (b) the corresponding simulated efficiency

\subsubsection{Gain Along the Circuit}

In order to determine where on the circuit energy is coupled, two methods were proposed. The first method measures the voltage and current on the circuit along the length of the circuit. In this way the power can be calculated at any location. The second method measures the electric field in the interaction region near the circuit. This method assumes that as the RF wave in the meander line circuit is increasing, the alternating electric field component near the circuit will also increase. This approach is similar to the method used at $\mathrm{NU}[14,15]$ where the RF field amplitude was measured in the interaction space. That experiment indicated that gain occurs on the first half of the circuit, as shown in Sec. 2.8.3.

\section{Method One: Voltage and Current Diagnostics}

This method uses both the current and voltage to determine the power along the length of the circuit. To find the current, the VSim B-Loop diagnostics encircle the 
line in the center of the slow wave circuit at multiple locations along the length. The voltage is determined at these same locations using a psuedopotential diagnostic from the center line to the ground plane. The power is found by multiplying the current by the voltage. Fig. 7.18 shows the gain along the length of the circuit for $V_{a s}=1550 \mathrm{~V}$, $I_{\text {beam }}=150 \mathrm{~mA}, L_{s w}=15 \lambda_{s w}, V_{c s}=400 \mathrm{~V}$, and $V_{b e}=100 \mathrm{~V}$. Note that the circuit is much longer than the experiment to easily monitor the gain along the length and to compare the simulated gain with the theoretical gain predicted by Pierce theory. The simulated gain along the circuit follows Pierce theory rather well until saturation starts at around 14 wavelengths. Saturation occurs when electrons collect on the slow wave circuit as the give up energy. The electron beam becomes depleted further down the tube, and with no energy left in the electron beam, the gain saturates.

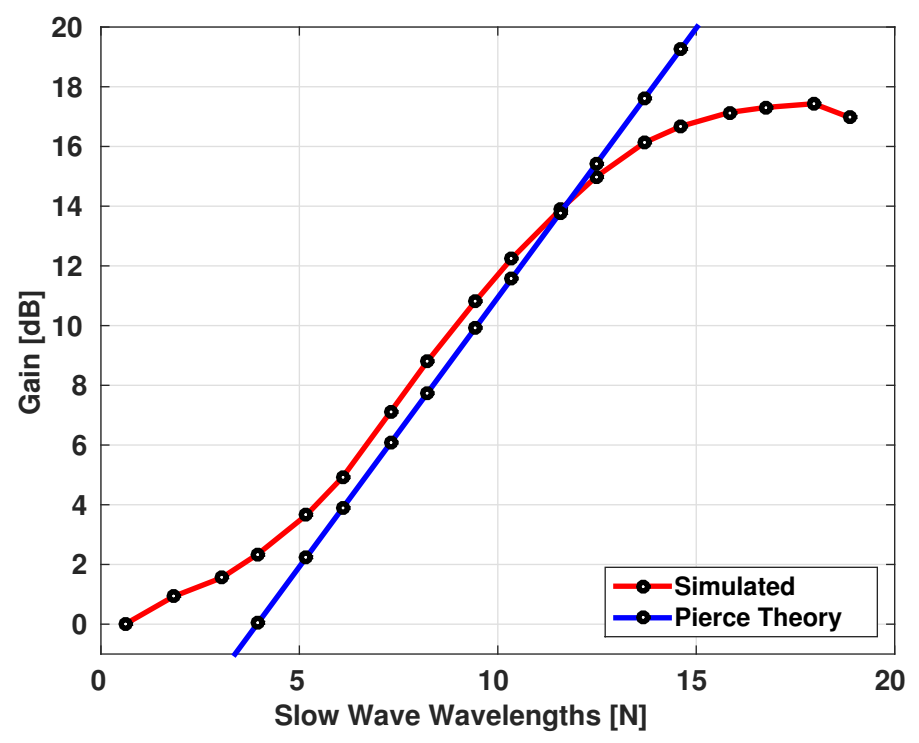

Figure 7.18: Gain along the length of the circuit for both VSim simulation (red), and Pierce theory (blue). 


\section{Method Two: Electric Field Near The Circuit}

This approach uses the RMS value of the AC coupled $y$-component of the electric field near the circuit along the length of the circuit. A fieldOnLine history diagnostic is used along the length of the circuit to implement this method. Figure 7.19 shows the AC RMS electric field from $0.625 \mathrm{~cm}$ and $1.25 \mathrm{~cm}$ away from the circuit, along the center of the circuit ( $z$-direction) for $V_{a s}=1550 \mathrm{~V}, I_{\text {beam }}=150 \mathrm{~mA}, L_{s w}=18 \lambda_{s w}$, $V_{c s}=400 \mathrm{~V}$, and $V_{b e}=100 \mathrm{~V}$. This circuit is three times longer than the NU experiment, which is only $6 \lambda_{s w}$ long. Each plot shows periodic variations at half of the slow wave wavelength due to the standing wave pattern. The edges $(N=0$ and $N=18 \lambda_{s w}$ ) show electric field values at zero or approaching zero as the diagnostic gets closer to the boundary condition at the edge of the domain. Fig. 7.19(a) shows the electric field increasing at a rather constant rate; whereas Fig. 7.19(b) shows a more sporadic increase in the electric field.

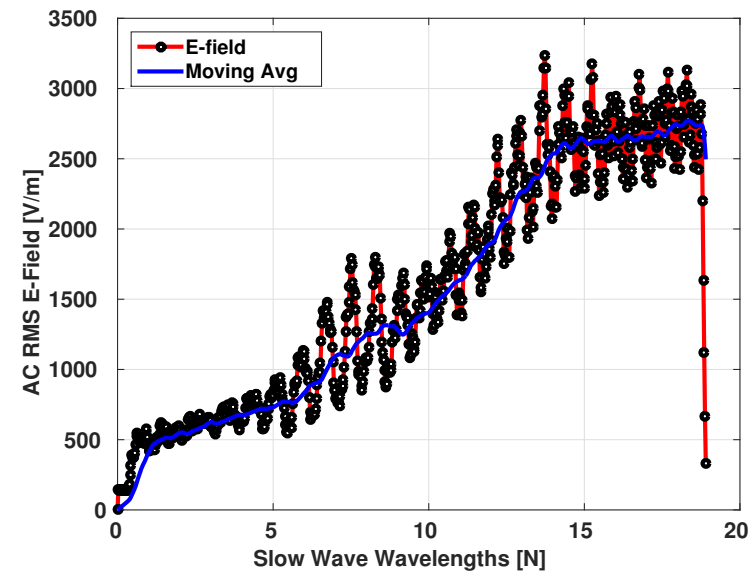

(a)

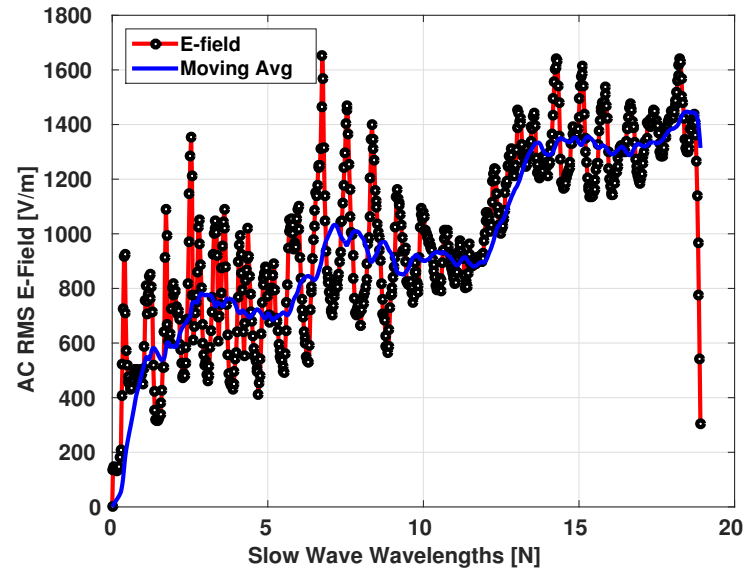

(b)

Figure 7.19: The AC coupled RMS $y$-component of the electric field, in red, and the moving average, in blue, along the length of the circuit for (a) $0.625 \mathrm{~cm}$, and (b) $1.25 \mathrm{~cm}$. 
To determine the gain from the electric field plot, Eq. (7.4) is used where $E_{\text {in }}$ and $E_{\text {out }}$ are the electric fields at the beginning and end of the tube, respectively. The gain determined from Fig. 7.19 (a) is $14 \mathrm{~dB}$ using $E_{\text {in }}=500 \mathrm{~V} / \mathrm{m}$ and $E_{\text {out }}=$ $2500 \mathrm{~V} / \mathrm{m}$. The gain determined from Fig. $7.19(\mathrm{~b})$ is $9 \mathrm{~dB}$ using $E_{\text {in }}=500 \mathrm{~V} / \mathrm{m}$ and $E_{\text {out }}=1400 \mathrm{~V} / \mathrm{m}$.

$$
\text { Gain }=20 \log \left(\frac{E_{\text {out }}}{E_{\text {in }}}\right)[\mathrm{dB}]
$$

\subsubsection{Discussion}

Both examples of the gain vs. circuits of different lengths shown in Figs. 7.16 and 7.17 show that the gain increases as the length is increased and that the gain starts to level off at some length. The reason for the gain leveling is due to limited available energy in the beam to extract, so the simulated gain levels off. The simulated gain in Fig. 7.16(a) follows the theoretical gain closely until the length is about 15 wavelengths where the gain starts to level off. This is the point at which there is almost no more available energy in the beam to extract. Note that the efficiency at this point is about $20 \%$, which is a rather low efficiency. The simulated gain in Fig. 7.17(a) deviates from the theoretical gain immediately after 6 wavelengths. Because the gain of the device is consistent for many powers, more energy is extracted from the beam at higher powers, and at shorter lengths the beam power is almost all depleted. Note that this starts to happen at $18 \%$ efficiency. So when around $20 \%$ of the beam power is extracted, extracting more energy from the beam becomes more difficult. Basically

this result shows that as long as there is energy left in the beam, the gain will continue to increase as the length of the circuit is increased. 
The reason that the efficiency is so low for this device, even with the longer lengths, is because of the large cycloid radius of the electron beam. An electron with a large cycloid radius gives up less potential energy before getting collected than an electron with a small cycloid radius. Figure 7.20 shows an example of two trajectories with different radii. The red trajectory has twice the radius as the blue. As the electron travels down the tube, potential energy is extracted by the RF wave on the circuit, and the trajectory moves closer to the SW circuit on each cycloid until it collects on the SW circuit. With a larger cycloid radius, the electron collects sooner, and less energy is extracted, indicated by the estimated energies $E_{r 2}<E_{r 1}$. The bottom of the trajectory is a convenient place to compare how much energy is lost by the electron because the electron has no kinetic energy. The closer this point is to the SW circuit, the more potential energy the electron has given up to the RF wave. The electron in red gives up some energy to the RF wave, and collects on the circuit with a high kinetic energy, which is lost. The smaller cycloid radius allows the electron give up more energy to the RF wave, and collects on the SW circuit with less kinetic energy than the electron with the larger cycloid radius. The smaller cycloid radius is more efficient but is also farther away from the circuit at first, so the interaction impedance is lower. The energy extraction may take a longer distance with smaller cycloid radii, but will be more efficient with a sufficient interaction region length. 


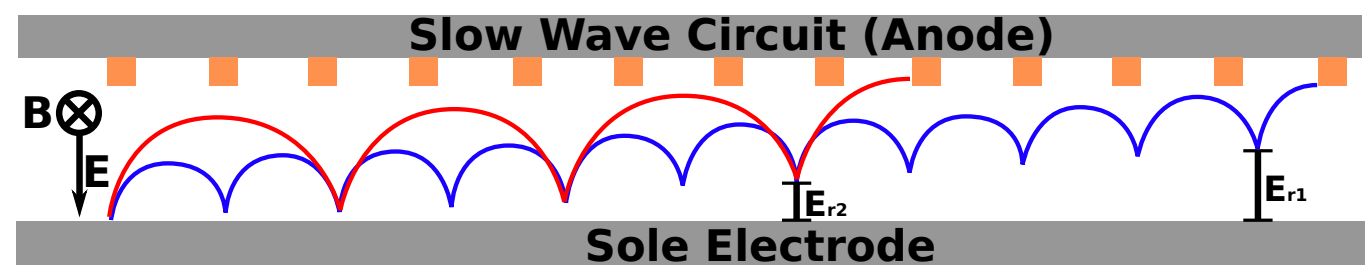

Figure 7.20: Two electron trajectories with different cycloid radii. The blue trajectory has half the cycloid radius than the red trajectory. $E_{r 1}$ and $E_{r 2}$ are the estimated energy extracted from the smaller and larger radii, respectively.

The plot of the gain along the length of the circuit shown in Fig. 7.18 strongly resembles the plot of gain vs. the length of the slow wave circuit in Fig. 7.16. The explanations on the plot behavior in Fig. 7.18 are the same as the explanations of Fig. 7.16. This result shows that the beam energy coupling to RF wave on the circuit happens along the entire length and follows Pierce theory until little available energy is left in the beam. This contradicts the observation in [15] where the energy extraction happens on the first half of the circuit. The conclusion that the gain occurred in the first half of the circuit was due to the electric field measurements observed near the circuit during operation, shown in Fig. 2.33. This result was the motivation behind method two of studying gain along the circuit.

Measuring the gain using the electric field near the circuit somewhat follows the true gain along the circuit when measured very close to the circuit, but it greatly deviates when measured farther away. Figure 7.19(a) shows the electric field gain increasing along the length until it starts to level out at 14 wavelengths, which matches the general shape of the gain plot in Fig. 7.18. The magnitude of the final gain as measured by the method, $14 \mathrm{~dB}$, does not match the actual gain of $18 \mathrm{~dB}$. Also, as the electric field measurement moves farther away from the circuit, as shown in Fig. 7.19(b), the shape and the gain start to deviate greatly. This result indicates the 
unreliability of using this method to determine the gain along the length of the circuit. When the measurement is close to the circuit, the measurement is more reliable.

The unreliability in measuring the gain along the circuit using method two is most likely the reason for the result that the gain occurred in the first half of the circuit in the NU experiment, shown in Fig. 2.33. The probe used to measure the RF power in the tube was about $1 \mathrm{~cm}$ long, and the dielectric sheath was about $1 \mathrm{~cm}$ in diameter. Given the size of the probe, the RF measurements were not very close to the circuit and could result in this unreliable behavior.

\subsubsection{Summary}

This section determined the reliability of two methods to determine the gain along the length of the circuit and in determining the RF signal amplification along the length of the circuit. The gain along the circuit follows Pierce theory as long as there is enough energy in the beam. The result found in the NU paper, where the gain occurs in the first half of the circuit, is possibly incorrect due to the unreliability in the electric field measurements near the circuit to indicate gain on the circuit. The best method to determine the gain along the circuit uses voltage and current measurements on the circuit. The electric field measurements near the circuit are found to be approximate, but only if the measurement is very close to the circuit. The result can accumulate a significant of error if it is not close.

\subsection{Distributed Cathode Approximation Studies}

To test the validity of the cathode approximations described in Sec. 5.3.6, a series of studies were performed. The first thing to determine is the effect the approximation 
has within the simulation. The approximation should not introduce error to the model and should be transparent to the model. Also, the approximation parameters need to be optimized for gain. The simulation results for the three methods are described here.

\subsubsection{Cathode Approximation 1: Raised Cathode}

The study here is to determine the effect of the $E_{R F}=0$ region in the raised cathode approximation. This model is described in detail in Sec. 5.3.6. This method was found not to be a viable method, but the analysis is discussed here both for completeness and for the interesting result observed which gives some insight into CFA operation. Because the $E_{R F}=0$ region is far away from the circuit, it was thought to affect gain minimally, but it was actually found to have a profound affect on gain.

To see the effect of this approximation on the simulation, an injected beam variation of this setup was implemented with varying sizes of regions. For this test, identical to the injected beam setup, electrons are injected from a cathode potential, and the sole is set at a different potential. Fig. 7.21 shows a diagram of the study model. Note that the electrons can enter the $E_{R F}=0$ region. The implementation is almost the same as the injected beam case but with the added $E_{R F}=0$ region. The thickness of the $E_{R F}=0$ region is varied from 0-12 cells. The full run is simulated and the gain is studied. Fig. 7.22 shows the gain and the corresponding currents on the conducting boundaries vs. the number of cells used for the $E_{R F}=0$ region $\left(N_{r f=0}\right)$. 


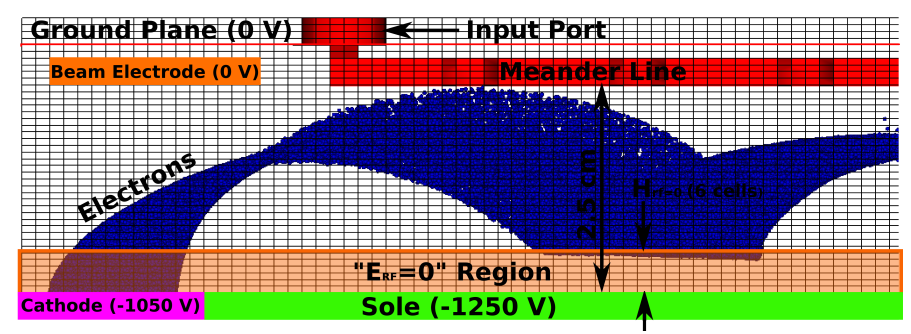

Figure 7.21: A variation of the cathode approximation method one to test the $E_{R F}=0$ region effects on gain. This variation emits electrons similarly to the injected beam configuration but there is the $E_{R F}=0$ region. Electrons are allowed to enter the region but no $R F$ fields are calculated there.

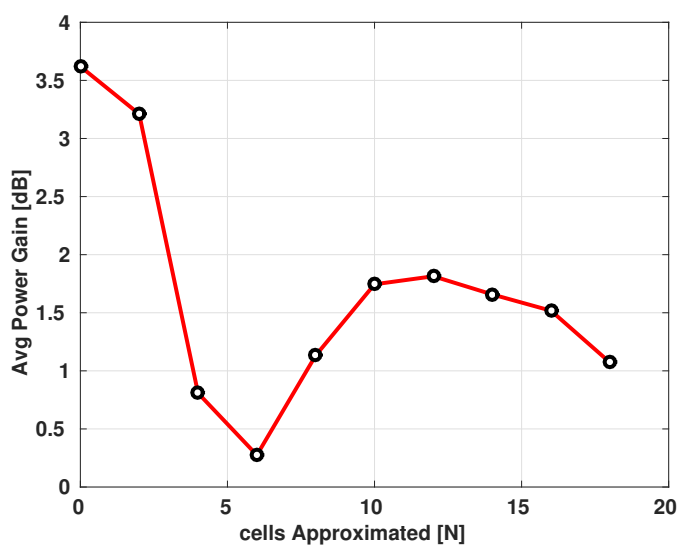

(a)

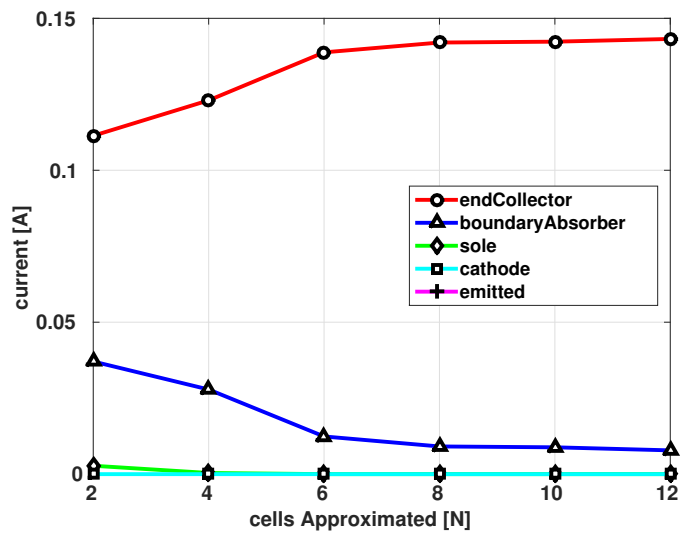

(b)

Figure 7.22: (a) The gain and the corresponding (b) currents vs. the conducting boundary region thickness.

The size of $E_{R F}=0$ region greatly affects the gain as seen in Fig. 7.22. The gain for the 0 cell case is only $3.5 \mathrm{~dB}$ rather than $4.2 \mathrm{~dB}$ observed in Figs. 7.11 and 7.9 because the beam electrode is $0 \mathrm{~V}$ instead of $200 \mathrm{~V}$. It was expected that with the size of the $E_{R F}=0$ region being small and being far away from the SW circuit, the gain would be very similar to the gain observed without the $E_{R F}=0$ region. Instead the gain drops dramatically at 6 cells. The assumption that electrons far away from 
the circuit do not interact with the RF wave on the circuit is incorrect. Electrons at the bottom of their trajectory spend much longer time there due to their low kinetic energy. RF waves and space charge have a large affect because of the long residence time. This observation shows the importance of the entire electron trajectory path, even far away from the circuit. Because of this sensitivity to even small $E_{R F}=0$ region heights, this approximation could not be used.

\subsubsection{Cathode Approximation Two: Segmented Cathode}

This method was found to be more sensitive to the cathode parameters and beam electrode potential rather than to the current distribution. Eventually this method was also abandoned. It is described here for completeness and to show the sensitivity of the beam injection parameters on gain. There are four variables that can be varied in order to optimize gain: the potential between the anode and sole $\left(V_{a s}\right)$, the potential between the cathode and the sole $\left(V_{c s}\right)$, the magnetic field $(B)$, and the length of the sole $\left(L_{s}\right)$. Three studies were performed to optimize the gain for this configuration, and these are outlined in the following sections. The first study was to determine which combination of $V_{a s}, V_{c s}$, and $B$ gave the best beam trajectory. The second study determined the emitter to emitter length, $L_{e 2 e}$, to maximize the current down the tube. The third study determined the best magnetic field for gain. The magnetic field chosen in the first study theoretically yields the optimum beam retardation for the SW circuit, but this last study ensures this assumption is correct and shows the sensitivity of the gain from magnetic field changes. 


\subsubsection{Trajectory Optimization}

This cathode approximation uses a beam electrode potential of $0 \mathrm{~V}$. Because many emitters will be directly under the SW circuit, the beam electrode was used to keep the electric field consistent between all of the emitters under the SW circuit and the region not directly below the SW circuit. Also, this beam injection technique is very inefficient when using same parameters as the injected beam. Because of the fringe electric fields at each injection point caused by the proximity of the sole sections to the cathode sections, with $V_{a s}=1250 \mathrm{~V}$, much of the beam collects on the $\mathrm{SW}$ circuit right away, and the CFA has low gain. Because beam optics can be used with injection points below the SW circuit, the only way to optimize the beam was to vary $V_{a s}, V_{c s}$, and $B$. To decrease the cycloid radius and keep the retardation constant, both $V_{A S}$ and $B$ were increased to keep the $E / B$ ratio constant. Decreasing the cycloid radius decreases current to the SW circuit. $V_{c s}$ controls the y-position of the guiding center of the electrons. Increasing $V_{c s}$ pushes the electron beam closer to the SW circuit; and theoretically, fewer electrons will be collected on the sole; the cycloid radius stays the same; and potentially more electrons will be collected on the SW circuit. By varying all three parameters, the beam can be pushed up towards the SW circuit, and the cycloid radius can be decreased so that the current does not all collect on the SW circuit to maximize the gain.

The emitter to emitter spacing also has an effect on the gain and makes optimization difficult. Ultimately the optimization requires a parameter sweep with a focus on the actual gain, but some of the intermediate optimization steps require a more holistic trial and error approach of how the electron trajectories look rather than just

optimizing gain. This holistic approach is required because of the interconnectivity 
of the cycloid radius and the emitter-to-emitter spacing, which is discussed in the following section. Through trial and error, the optimum anode-to-sole voltage was $V_{a s}=1650 \mathrm{~V}$ and the optimum cathode-to-sole voltage was $V_{c s}=300 \mathrm{~V}$. This combination of voltages allowed for the cycloiding electrons to travel close to the slow wave circuit without collecting. Fig. 7.23 shows the electron trajectories for $V_{a s}=1650 \mathrm{~V}$ and $B=6.8 \mathrm{mT}$ for different cathode-to-sole voltages. Note that with larger $V_{c s}$, shown in in Fig. $7.23(\mathrm{c})$, space charge becomes a problem for beam injection. This effect can be seen by the electrons spreading out right as they leave the cathode.

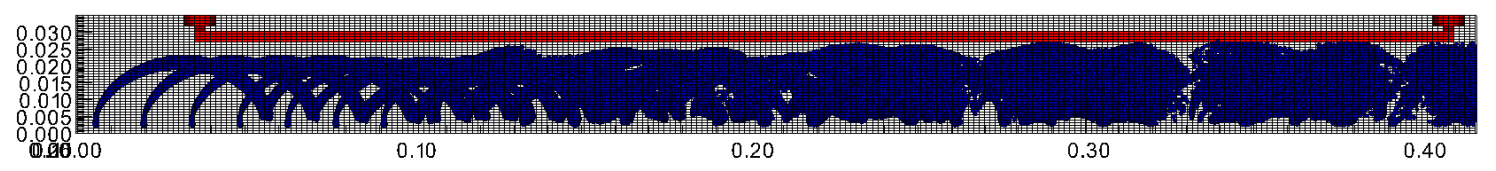

(a)

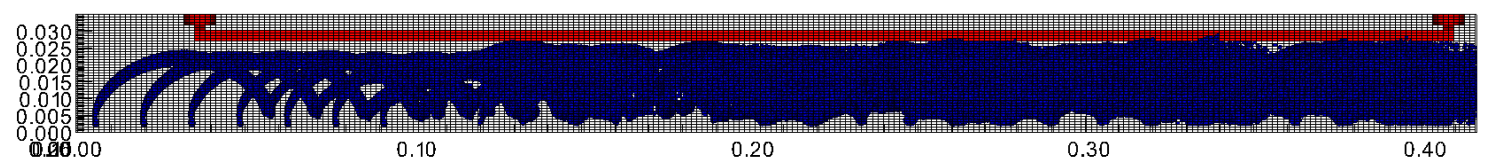

(b)

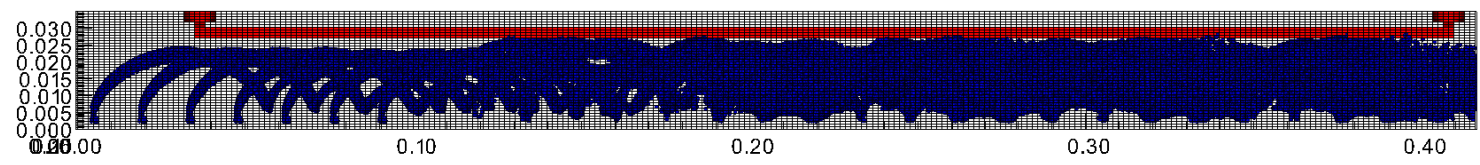

(c)

Figure 7.23: Electron trajectories for 7 emitters, $V_{A S}=1650 \mathrm{~V}, B=6.8 \mathrm{mT}$, and different values of $V_{c s}$, (a) $200 \mathrm{~V}$, (b) $300 \mathrm{~V}$, and (c) $400 \mathrm{~V}$.

By pushing the beam closer to the SW circuit, the beam-wave interaction should increase, thereby, increasing the gain. Another thing to note is that by increasing the anode-to-cathode voltage $\left(V_{a c}\right)$, the beam power is increased which can add to the 
gain as well. For accurate comparisons among simulations, this effect must be taken into account.

\subsubsection{Emitter to Emitter Spacing Study}

After determining the 'best' electron trajectory, the emitter spacing needs to be optimized to that trajectory. Each emitter in the interaction region creates a 'hole' in the barrier provided by the sole electrode. The emitter to emitter spacing needs to be chosen as to not coincide with the cycloid diameter. If the emitter-to-emitter spacing is an integer multiple of the cycloid diameter, the electrons will cycloid right back into the one of the following emitters. Figure 5.17 in Sec. 5.3.6 shows an example of when the cycloid diameter is equal to the twice the emitter-to-emitter spacing $\left(L_{e 2 e}=10\right.$ cells $)$, and most of the current sneaks through the sole potential barrier. Fig. 7.24 shows how sensitive the gain is to the emitter-to-emitter spacing for the optimum trajectory. The gain is directly correlated with the current to the end collector. With $L_{e 2 e}=10$ cells, the gain is minimized because the all the current is collected by the following emitters as shown in Fig. 5.17. With $L_{e 2 e}=11$ cells, the gain is maximized because the most of the current is transmitted to the end of the tube as shown by Fig. 5.19 on page 128 . 


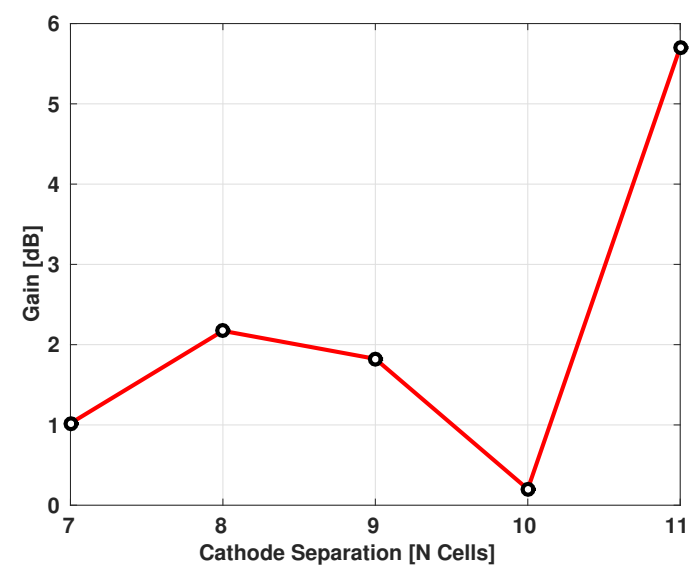

(a)

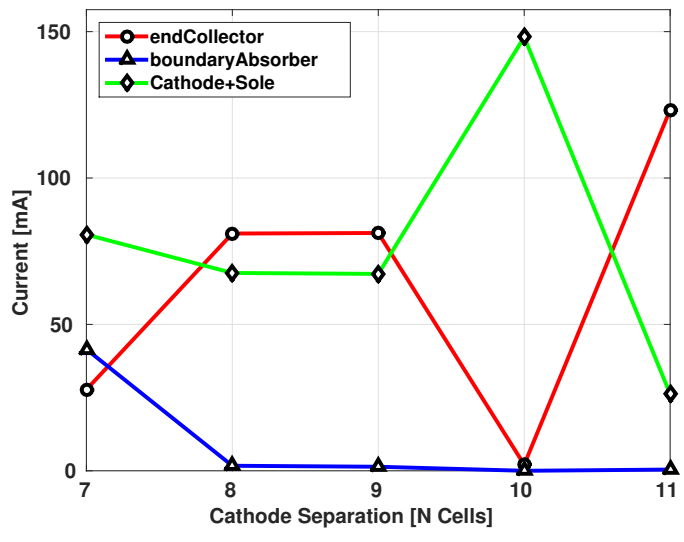

(b)

Figure 7.24: (a) The gain and the corresponding (b) currents vs. the cathode separation for $V_{a s}=1650 \mathrm{~V}$ and $B=6.7 \mathrm{mT}$.

\subsubsection{Magnetic Field Study}

To get full transmission of electron beam current down the tube is very difficult. The tube is rather long and contains $>20$ emitter potentials down the length. Finding an optimum condition is difficult with this many emitters, and generally that optimum point has a narrow margin. Using the optimum emitter-to-emitter length found in the previous section, the magnetic field is varied and the result is shown in Fig. 7.25. From $B=6.75 \mathrm{mT}$ to $B=6.78 \mathrm{mT}$ the gain goes from the maximum at $6 \mathrm{~dB}$ to the minimum at $-1 \mathrm{~dB}$. Note how the current shifts dramatically from small changes in magnetic field. The optimum gain point has such a small magnetic field window that it is impractical. Any small change in emitter current also varies the gain. This sensitivity prevents an accurate way to test different current distributions because the shifts in gain are mainly due to the small shifts in the electron trajectories, so this method was abandoned. 


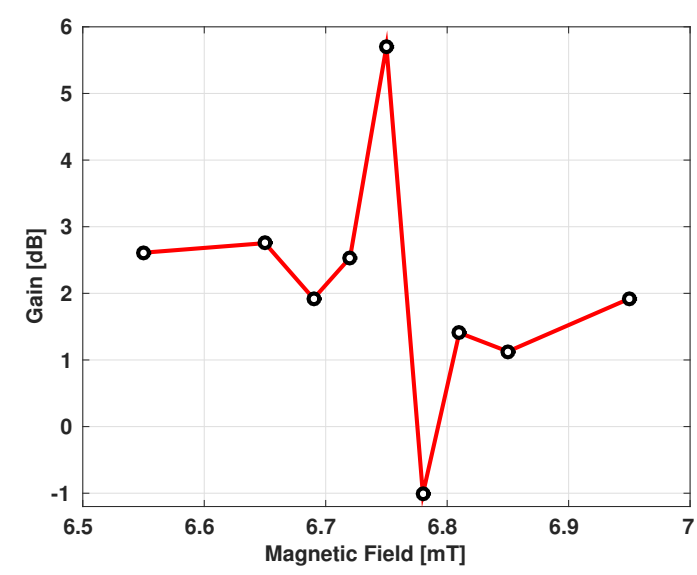

(a)

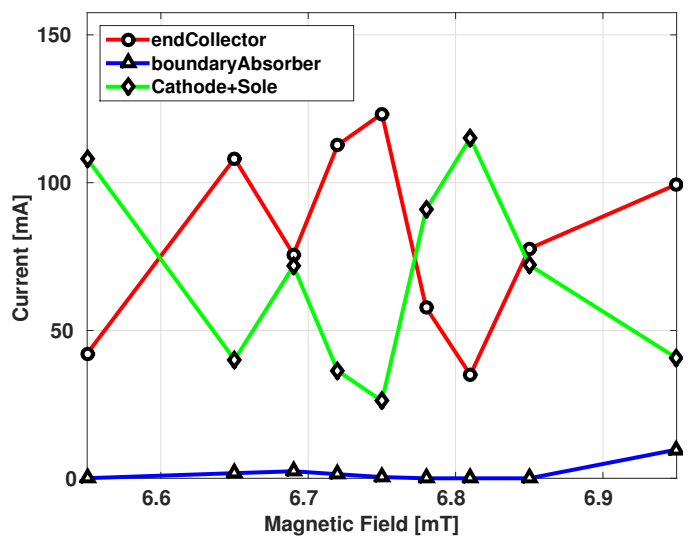

(b)

Figure 7.25: (a) The gain and the corresponding (b) currents vs. the magnetic field with $N_{c 2 c}=11$ cells for the cathode approximation 2 .

\subsubsection{Cathode Approximation Three: Raised Cathode With Approxi- mated Current}

This approximation is the one used to implement the distributed cathode. First, the approximation effects on the simulation are discussed. Second, a study on the optimization of the parameters with respect to gain is performed.

\subsubsection{Divergence Free Region Effects}

To see the effect of the divergence free region on the simulation results, a comparison was performed with and without the region. Because the electrons are emitted from within vacuum in the divergence-free cathode approximation, the comparison simulation also emits from within vacuum, but this case uses a conducting boundary condition at the point of emission. Fig. 7.26(a) shows the electron trajectories for the conducting boundary condition method, and Fig. 7.26(b) shows them for the divergence free method. The full simulation is run with particles and RF on the 
circuit to compare the gain of both implementations. The parameters used were $V_{a s}=1550 \mathrm{~V}, B=6.65 \mathrm{mT}, P_{r f}=1 \mathrm{~W}$, and $I_{\text {beam }}=150 \mathrm{~mA}$. These parameters were chosen based on a simple optimization, not shown here, where the current can travel down the tube close to the circuit with little current lost to the circuit. Because of the change in electron beam optics when using this approximation, the cycloid radius needs to be smaller so as not to collect on the circuit right away. Three different emitter lengths were used to have a more complete comparison. A more in depth study on the effect of emitter length on gain is performed in Sec. 7.6.1.2; here the focus is on the effect the approximation has on the simulation results.

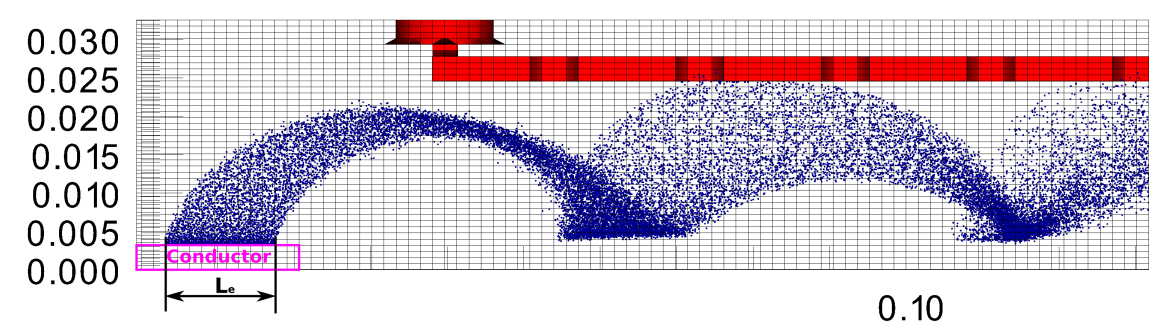

(a)

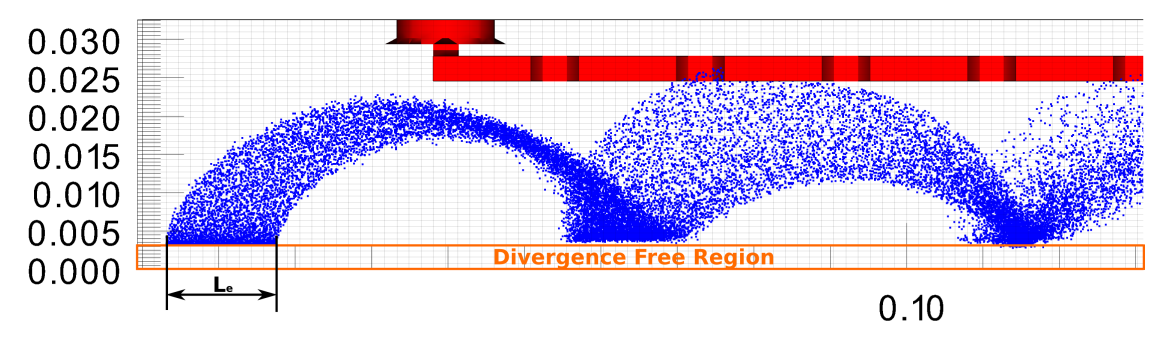

(b)

Figure 7.26: The two setups to determine the effect of the divergence free region. Emission from a (a) conducting region and from (b) the divergencefree region. 


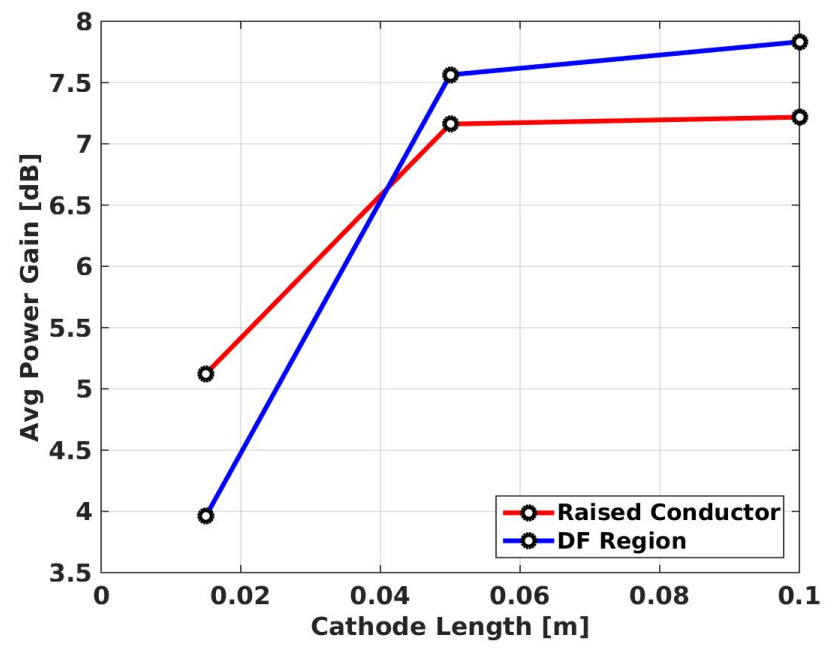

Figure 7.27: Gain vs. emission length, $L_{e}$, for the both divergence-free (DF) cathode approximation and the raised emitting conductor.

Fig. 7.27 shows that with $L_{e}=1.5 \mathrm{~cm}$ the divergence free approximation is $1 \mathrm{~dB}$ less than the conducting case, and at the larger lengths, the divergence free approximation is about $0.5 \mathrm{~dB}$ greater. The differences are attributed to the variation in space charge in the different methods and its effect on beam emission. As shown in the injected beam sensitivity study on beam optics, small differences in beam emission can cause relatively large differences in gain. These results show a small effect on gain when using the divergence free region, but an acceptable error. These differences affect the overall gain but will not affect the overall trends of gain from emitter length or the effects of the emitter current profile.

\subsubsection{Trajectory Optimization}

Just as it was found for the injected beam, the beam injection optics is very important to optimize gain. Since the majority of the distributed cathode is below the slow wave circuit, no beam electrode can be used to optimize the injection. To control the beam, 
only $V_{a s}$ and $B$ are used, and the beam electrode is set to $0 \mathrm{~V}$ in order to keep the electric field over all of the cathode consistent. The cathode to sole voltage, $V_{c s}$, is not used because in this cathode approximation, $V_{c s}$ is controlled by the divergence-free region height. To minimize the effect of the divergence-free region on the simulation, $V_{c s}$ is set to $200 \mathrm{~V}$ so that the region is small as possible while preventing electron beam current from back bombarding the sole electrode. Different values of $V_{a s}$ and $B$ are swept to find the optimum combination. The optimum retardation was found to be $R=31.9$ from the injected beam study in sec. 7.3.1, so the ratio between $V_{a s}$ and $B$ is kept constant to maintain this retardation. Figure 7.28 shows the gain and the corresponding currents as the anode to sole voltage is swept and $B$ is altered to maintain the correct $\mathbf{E} \times \mathbf{B}$ velocity. The maximum gain is observed at $V_{a s}=1550 \mathrm{~V}$ and $B=6.5 \mathrm{mT}$. Below this voltage too much of the current is lost to the slow wave circuit immediately after emission, and greater than this voltage, the beam loses gain as it goes farther away from the circuit. These are the parameters used for all of the distributed cathode studies. 


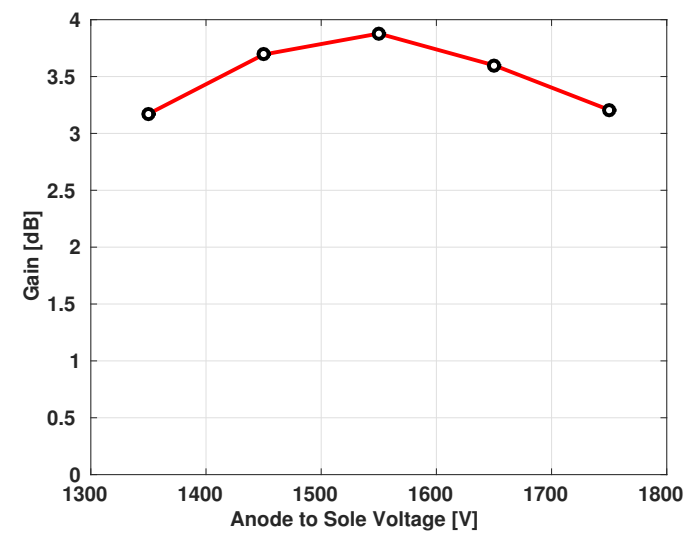

(a)

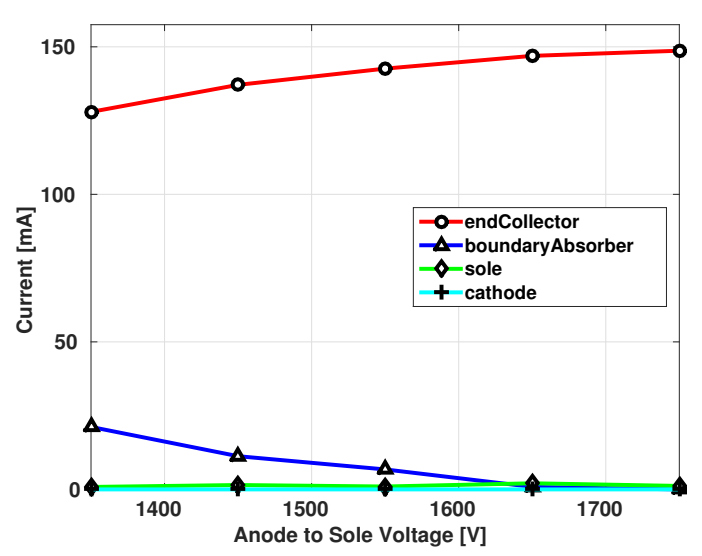

(b)

Figure 7.28: Optimization of the CFA parameters using the Divergencefree region showing the (a) gain and (b) currents vs. $V_{a s} . V_{c s}=200 \mathrm{~V}$ and the magnetic field is optimized for each voltage point to maximize gain.

\subsection{Distributed Cathode Studies: Static}

The goal of these studies was to show the effect on gain for different current distributions, both static and time-varying. Two studies are performed with static conditions: cathode length vs. gain and the current profile variation (uniform and linear profiles are used) vs. gain. In order to be synchronous with the RF fields on the circuit, the time-varying profiles are periodic in time and space. The chosen cathode approximation is method three: Raised Cathode With Approximated Current, and all results presented here are with this setup.

The simulation setup here is different than the NU setup. The main difference is that the slow wave circuit length is $50 \%$ longer than the NU one. The decision to elongate the circuit was made so that any benefit from a distributed cathode would be more observable. The intuition here was that with a longer circuit, gain along the length can saturate and current injected after the saturation point can be utilized. 
Also, the longer circuit gives more physical length for the distributed cathode. The disadvantage with using a longer circuit is longer simulation times, and these results cannot be compared directly with the experiment.

\subsubsection{Uniform Emission Profile}

The uniform profile uses a constant current density over a specific length. By lengthening the cathode and keeping the total current constant, the current density can be decreased. The hypothesis here is that with smaller current density at the input the noise from the beam can be reduced while, hopefully, maintaining the gain. Also, the smaller current density could allow for amplification of smaller RF input fields on the circuit by preventing the electron beam from generating too much noise.

The effect of emitter length and the magnitude of the RF input power on three different parameters (gain, noise, and efficiency) were studied. The first study looked at the gain variation with the magnitude of the RF input power for each emitter length. The next study took this same data, but looked at the gain versus the emitter length for each RF input power.

\subsubsection{RF Power Study}

Just as in Sec. 7.4.2, the RF input power was swept, and the gain and SNR were determined, now using the distributed cathode approximation and different cathode lengths. Figure 7.29 shows the gain and SNR vs. the RF input power for three different cases: the injected beam configuration from Sec. 7.4.2 and the distributed beam configuration with 10 and $20 \mathrm{~cm}$ emitter lengths. To determine the gain, the PSD method was used as described in Sec. 7.4.2.3. The injected beam configuration shows the highest gain for all RF input powers, and as the cathode length increases, 
the gain decreases. Once again, the small deviation in gain at the lowest RF input powers is caused by noise in the PSD method to calculate gain. The signal-to-noise ratio for all cathode lengths overlap and increase with the RF input power.

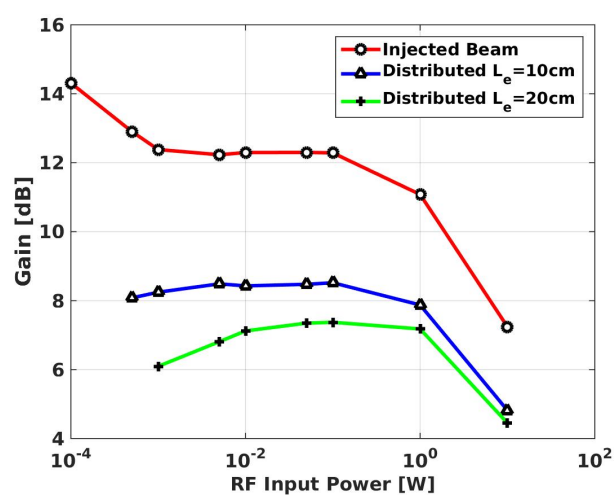

(a)

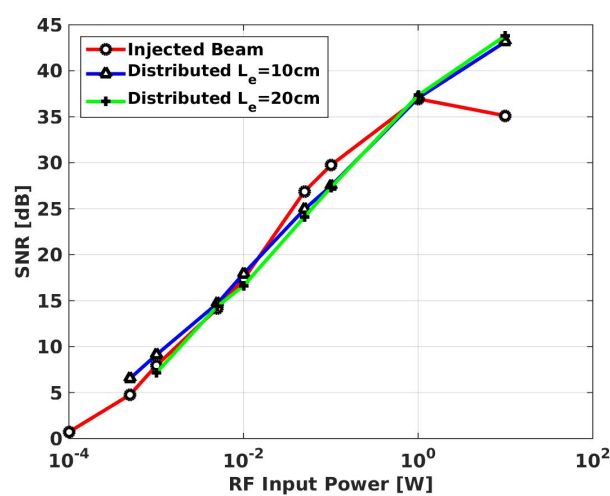

(b)

Figure 7.29: (a) The gain and the corresponding (b) SNR vs. the RF input power for the injected beam configuration wit a $1.5 \mathrm{~cm}$ emitter length and the distributed cathode configuration with $10 \mathrm{~cm}$ and $20 \mathrm{~cm}$ emitter lengths.

\subsubsection{Emitter Length Study}

Using the distributed cathode approximation, the length of the emitter was varied for various RF input powers. Figure 7.30 shows the gain and SNR vs. the emitter length for $P_{r f}=0.1 \mathrm{~W}$ and $I_{\text {beam }}=150 \mathrm{~mA}$. The gain initially increases as the cathode length increases until $L_{c}=10 \mathrm{~cm}$, and then the gain decreases. The SNR ratio remains relatively constant for all but the shortest cathode length $L_{c}=1.5 \mathrm{~cm}$. Only one RF input power for the emitter length study is displayed here because all RF powers showed the similar results. 


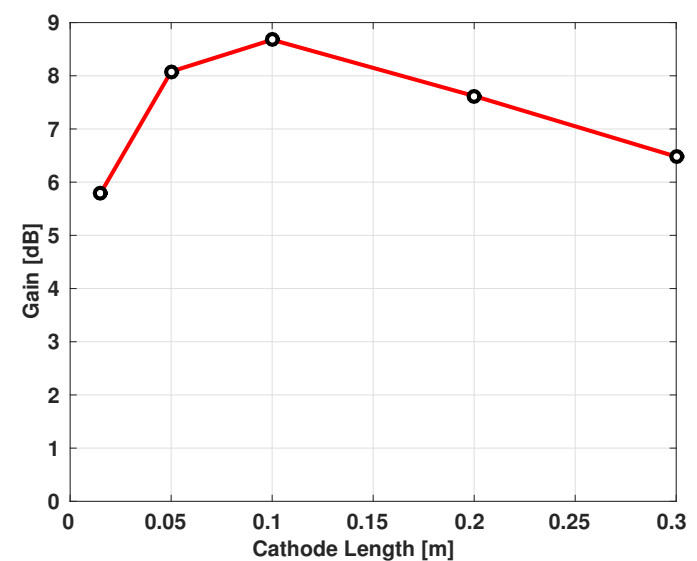

(a)

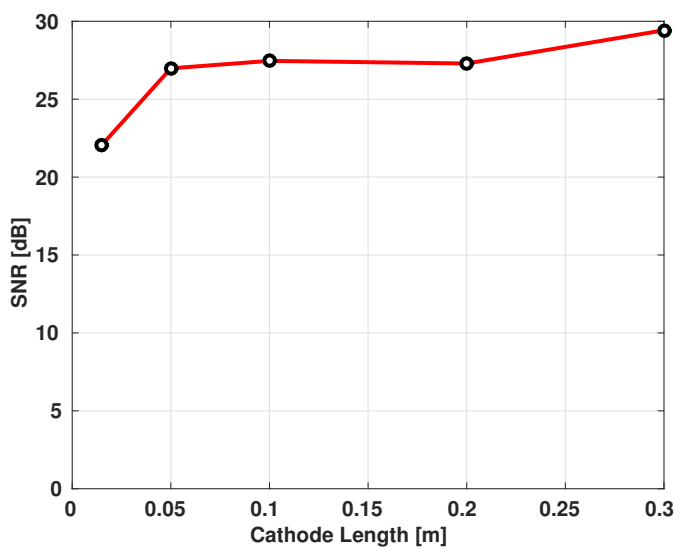

(b)

Figure 7.30: (a) The gain and the corresponding (b) SNR vs. the cathode length for the distributed cathode approximation with $P_{r f}=0.1 \mathrm{~W}$

\subsubsection{Beam Current Study}

One advantage of the uniform distribution is that since the area of the cathode is larger than the emission area of the injected beam cathode, more current can be injected due to the reduced space charge. Figure 7.31 shows the gain and corresponding currents for different beam currents using $L_{e}=20 \mathrm{~cm}$ and $P_{r f}=0.1 \mathrm{~W}$. As expected, with more current, the gain increases. 


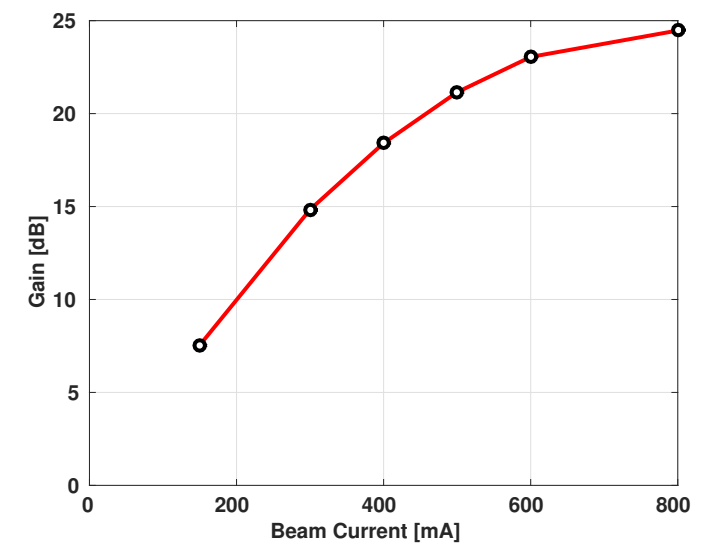

(a)

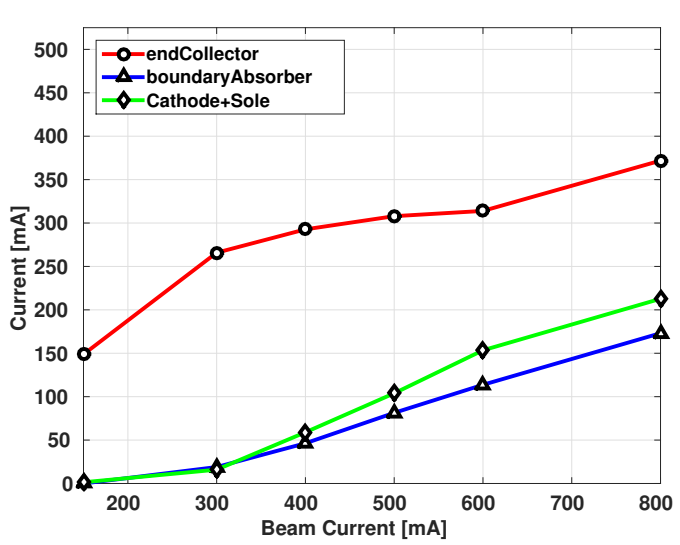

(b)

Figure 7.31: (a) The gain and the corresponding (b) currents vs. the beam current for the uniform emission profile with $L_{e}=20 \mathrm{~cm}$ and $P_{r f}=0.1 \mathrm{~W}$.

\subsubsection{Discussion}

As shown in Fig. 7.29, as the RF input power decreased for all cathode lengths and the gain levels out at the theoretical gain point predicted by Pierce theory. The behavior is similar to the injected beam RF power study as described in Sec. 7.4.2. At RF input powers greater than $10^{-1} \mathrm{~W}$ the gain is limited by the energy available in the beam, and at powers less than $10^{-2} \mathrm{~W}$ the gain deviates from the theoretical gain point due to the inaccuracies of determining the power.

An important correlation is that the gain decreases with longer emitters. Figure 7.30 (a) shows the decrease in gain from emitter length $10 \mathrm{~cm}$ to $30 \mathrm{~cm}$. The reason for the gain decrease in this region is that current injected farther down the tube is less effective than current injected at the beginning. Current that is injected farther down the tube has shorter distance (time) to interact with the RF wave. Figure 7.30(a) also shows the gain increasing from $1.5 \mathrm{~cm}$ to $10 \mathrm{~cm}$. This result has less to do with the emitter length and more to do with beam injection optics. At these small emitter 
lengths, space charge is significant enough to deflect the beam, and without proper beam injection optimization, the gain decreases. The main result is that as long as space charge is not significant, the gain of the device decreases with longer emitters.

Another interesting result is that the SNR is almost identical for all emitter lengths at all powers. Figure 7.30(b) shows no improvement to SNR from increasing the emitter length. By increasing the emitter length, the beam noise level is indeed lowered due to the reduced current density, but the gain is also reduced, so the SNR remains constant. So any noise reducing benefits from elongating the emitter is counteracted by the reduced gain.

Increasing the length of the emitter while keeping the current constant gives no benefits to the CFA design, but the longer cathode does allow for more beam current to be injected into the device due to the reduced space charge. Figure 7.31 shows that the gain increases with more current. Making the emitter length longer will decrease the efficiency of the device, but with greater injected current, the gain will increase.

\subsubsection{Linear Emission Profile}

Different linear profiles were studied in this work for different cathode lengths. The results were consistent with the results found in Sec. 7.6.1 and are not presented here for brevity. To summarize, the more current emitted farther down the tube, the less the gain. Elongating the emitter does reduce the noise level, but this improvement is counteracted by the decrease in gain.

\subsubsection{Summary}

Increasing the length of the distributed cathode offers little benefit to the linear format CFA operation. The gain decreases with emitter length, and there is no change to 
the SNR ratio. One benefit is that a longer cathode will allow for more injected beam current, which will increase the gain of the device, but at a cost of less efficiency. The "best" linear format CFA design, one with the highest gain and efficiency, is found to be a well designed injected beam model.

\subsection{Distributed Cathode Studies: Time Varying}

Changing the shape of the electron emission was found not to benefit the CFA, but modulating the emitters synchronously with the RF wave improves the gain and the SNR. The goal with the time-varying fields is to inject current synchronous with the retarding electric field created on the circuit to maximize beam energy coupling to the RF wave. Two different time-varying current profiles were tested: a sine wave and square pulse profile. The first study on these time-varying profiles is the effect of changing the relative phase between the RF wave and the beam profile. The next studies focus on the effects of the profile parameters, the RF input power, and emitter length. In the simulation, modulating the cathode does not consume any power; however in reality the GFEA modulation does consume power. Using the cathodes described by Guerrera et. al. [25], the power consumed by modulating the cathode for $I_{\text {beam }}=150 \mathrm{~mA}$ is $<1 \mathrm{~W}$ (see Sec. 2.6.3.2 for modulated power discussion).

Given the relatively low power of the device, $P_{\text {out }} \approx 100 \mathrm{~W}$, with $J_{\text {beam }}=10 \mathrm{~mA} / \mathrm{cm}^{2}$, the $\mathrm{RF}$ power needed to modulate the GFEA cathode is relatively high, $1 \%$ of $P_{\text {out }}\left(P_{\text {fea }} \approx 1 \mathrm{~W}\right)$, and decreases the gain significantly. For higher power devices, $P_{\text {out }}>10 \mathrm{~kW}$ with $J_{\text {beam }}>1 \mathrm{~A} / \mathrm{cm}^{2}$, the RF power needed to modulate the cathode is very conservatively estimated to be $P_{\text {fea }}<5 \mathrm{~W}$ which is $<.05 \%$ of $P_{\text {out }}$. Two different gain calculations are shown for the modulated cathode. For one calculation, the power 
dissipated by the GFEA is conservatively estimated to be $P_{f e a}=1 \mathrm{~W}$. Because of the exponential relationship between the output current and the gate-to-emitter voltage, the use of a GFEA cathode scales very well with high power devices. Instead of scaling up the output power of the NU CFA, which is time consuming, the estimated power consumption of the cathode is scaled down to show how the gain would be improved for a higher power CFA device. For the next gain calculation, the power dissipated by the FEA is estimated to be $P_{f e a}=0.1 \mathrm{~W}$. This representation of gain is shown as an example of how the gain might look like for a higher power device.

\subsubsection{Sine Wave Emission Profile Results}

\subsubsection{Phase Alignment}

The first study is to determine the best phase alignment between the current profile and the RF wave. The sine wave profile is described in Sec. 5.3.7, and the electron current density profile is give by eq. (5.3). At $f=150 \mathrm{MHz}$, the RF wave retardation is $R=31.9$. The $E \times B$ velocity and $\beta$ are adjusted to be synchronous. With $\phi_{\text {offset }}=0$ rad the current profile peaks correspond to the accelerating $E_{R F x}$ fields, see Fig. 5.25 for visualization. Figure 7.32 shows the gain and SNR for the $\phi_{\text {offset }}$ sweep with $L_{e}=30 \mathrm{~cm}, P_{r f}=1 \mathrm{~W}$, and $I_{\text {beam }}=150 \mathrm{~mA}$. The gain is highest when the current density peaks correspond to the decelerating $E_{R F x}$ fields, $\phi_{\text {offset }}=\pi \mathrm{rad}$. Also, the gain never goes below $6 \mathrm{~dB}$, which indicates that the modulated beam is overpowering the RF input signal on the circuit. The SNR varies from 35 to $45 \mathrm{~dB}$ in a somewhat random fashion. 


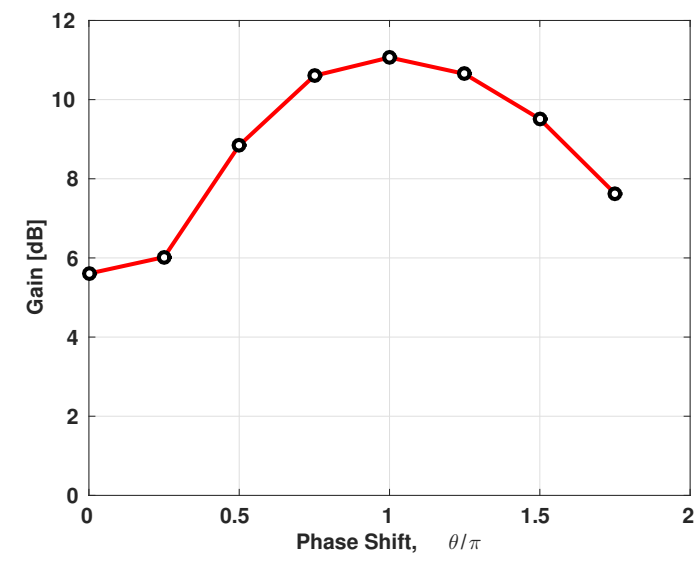

(a)

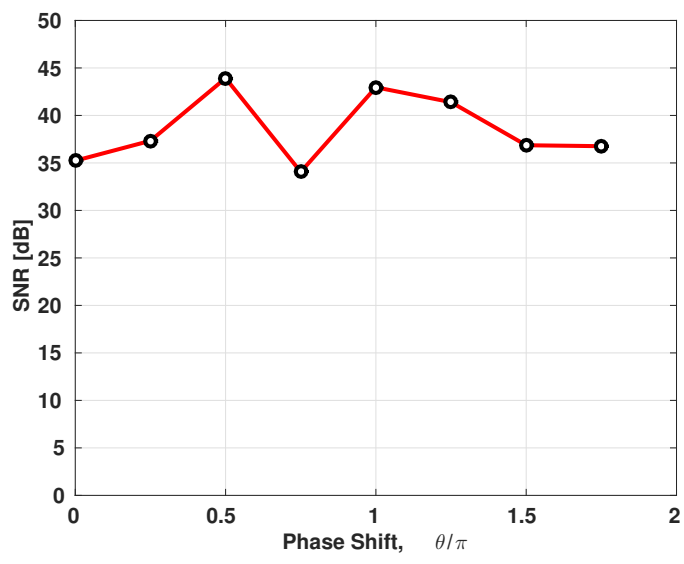

(b)

Figure 7.32: (a) The gain and the corresponding (b) SNR vs. the phase difference between the beam profile and the $\mathbf{R F}$ wave for $L_{e}=30 \mathrm{~cm}, P_{r f}=$ $1 \mathrm{~W}$, and $I_{\text {beam }}=150 \mathrm{~mA}$.

\subsubsection{Emitter Length}

The next study looks at the relationship between gain and the emitter length. While keeping the total average current constant at $I_{\text {beam }}=150 \mathrm{~mA}$, the emitter length is swept from 1.5 to $30 \mathrm{~cm}$. As the emitter length gets longer, the current density of profile peaks decrease but there are more of them. Figure 7.33 shows the gain and the corresponding currents of the emitter length sweep for the sine wave profile with $P_{r f}=1 \mathrm{~W}$ and $\phi_{\text {offset }}=\pi$. The gain in Fig. 7.33(a) shows a sharp increase in gain from 1.5 to $5 \mathrm{~cm}$ and a gradual decrease from 5 to $30 \mathrm{~cm}$. The boundary absorber current in Fig. 7.33(b) shows a sharp increase from 1.5 to $5 \mathrm{~cm}$ and then a gradual decrease from 5 to $30 \mathrm{~cm}$. The current lost to the cathode/sole region ranges from 40 to $60 \mathrm{~mA}$ for all lengths, which is quite large. 


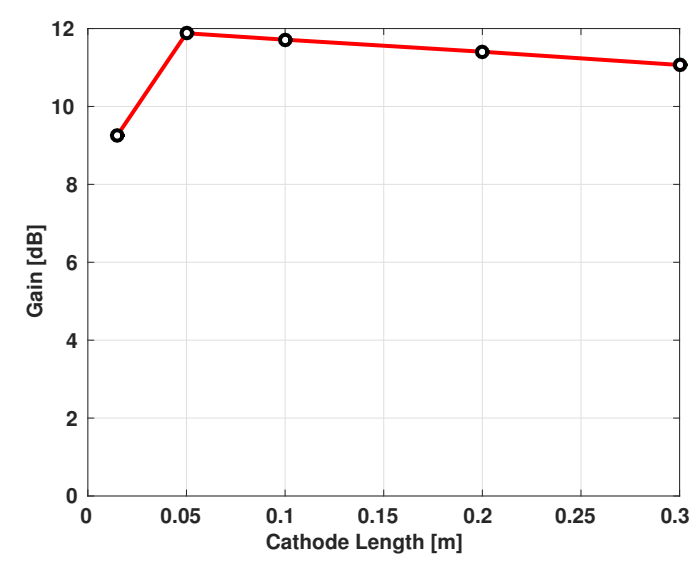

(a)

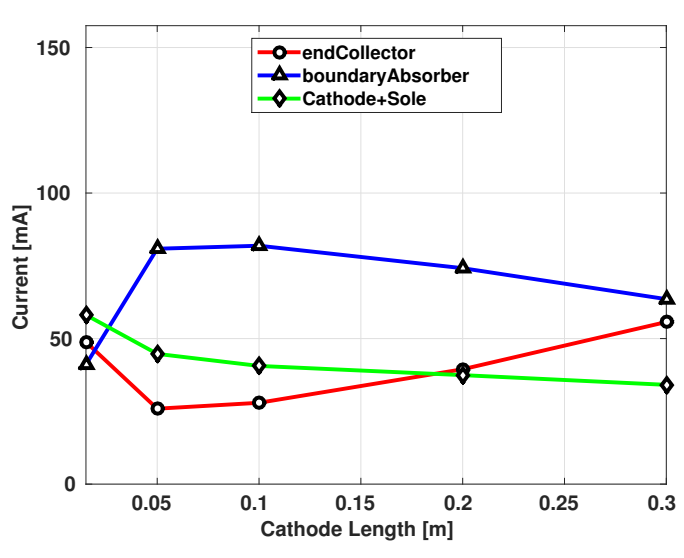

(b)

Figure 7.33: (a) The gain and the corresponding (b) SNR vs. the phase difference between the beam profile and the RF wave for $L_{e}=30 \mathrm{~cm}, P_{r f}=$ $1 \mathrm{~W}$, and $I_{\text {beam }}=150 \mathrm{~mA}$.

\subsubsection{Injected Beam Using Sine Wave Profile}

This study explores the effects of using a sine wave profile but with an injected beam configuration. The previous results using the sine wave profile were used with the divergence-free region and with the beam electrode set to $0 \mathrm{~V}$. This configuration uses a designated cathode section, a sole section and uses a beam electrode. The modulated cathode, since electrons are emitted for shorter periods of time than the continuous current injected model, has a higher peak current density and the emitter length is increased from $1.5 \mathrm{~cm}$, used for all previous injected beam simulations, to 3. $\mathrm{cm}$ in order to minimize space charge effects. Everything else is kept the same, $V_{a s}=1550 \mathrm{~V}, V_{c s}=400 \mathrm{~V}, V_{b e}=100 \mathrm{~V}$. Only one parameter sweep was performed on this beam injection type, which was the effect of RF input power. The results are not in this section, but are found in Sec.7.7.3.3 in Figs. 7.38 and 7.39 so that the results can be easily compared with the other beam injection methods. 


\subsubsection{Square Pulse Emission Profile Results}

Some of the studies on the square pulse profile are redundant with the sine wave profile but show some slight differences. The rest of the studies look at the physics of the modulation, which can be applied to the sine wave profile as well.

\subsubsection{Phase Alignment}

The phase alignment study is repeated and shown in Fig. 7.34 for the same parameters and the emitter pulse $L_{p}=1 \mathrm{~cm}$. The gain also peaks at $\phi_{\text {offset }}=\pi \mathrm{rad}$, but the difference between gain of in phase and out of phase is only $2 \mathrm{~dB}$ whereas the difference for the sine wave profile is $6 \mathrm{~dB}$. And the gain using the square profile is greater than the gain from the sine wave profile. Also, the SNR for the square wave profile is about the same in magnitude for the range in phase shifts, but looks less random than the sine wave profile.

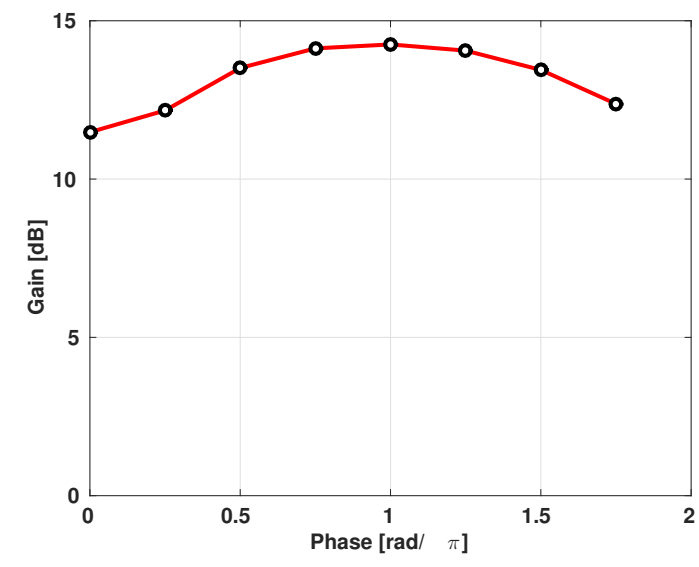

(a)

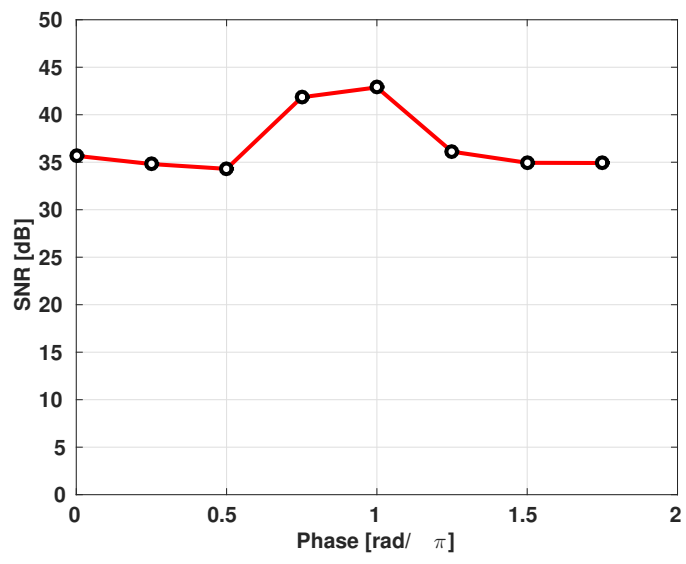

(b)

Figure 7.34: (a) The gain and the corresponding (b) SNR vs. the phase difference between the beam profile and the $\mathbf{R F}$ wave for $L_{e}=30 \mathrm{~cm}, P_{r f}=$ $1 \mathrm{~W}$, and $I_{\text {beam }}=150 \mathrm{~mA}$. 
The result where the gain does not go to zero even when the electron beam profile is out of phase with the RF wave on the circuit indicates that the modulated beam is driving the signal on the circuit rather than amplifying it. Figures 7.35 and 7.36 show both the phase and the gain along the circuit for $\phi_{\text {offset }}=0 \mathrm{rad}$ and $\phi_{\text {offset }}=\pi \mathrm{rad}$, respectively. To determine the phase shift, the $\mathrm{RF}$ voltage on the circuit is monitored and compared with the voltage of a test case where there is no electron beam. Figure 7.35(a) shows the phase of the RF signal shifting from 0 to $0.9 \pi \mathrm{rad}$. This means that the phase shifts from the RF input phase to close to the beam phase, which is offset by $\pi \mathrm{rad}$. The gain along the circuit in Fig. $7.35(\mathrm{~b})$ shows a decrease from 0 to 3 wavelengths and then a increase to the end of the circuit. Since with $\phi_{\text {offset }}=\pi \mathrm{rad}$ the beam and RF signal are in phase, there is only a small phase shift observed in 7.36(a), and the gain immediately starts increasing, as shown in 7.36(b).

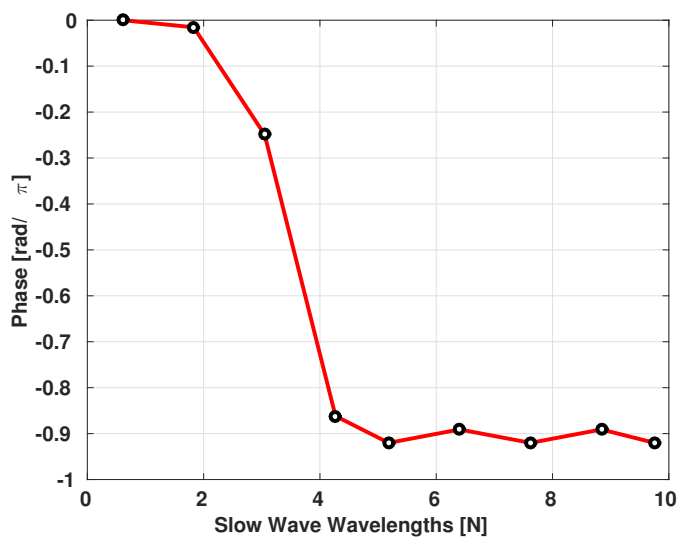

(a)

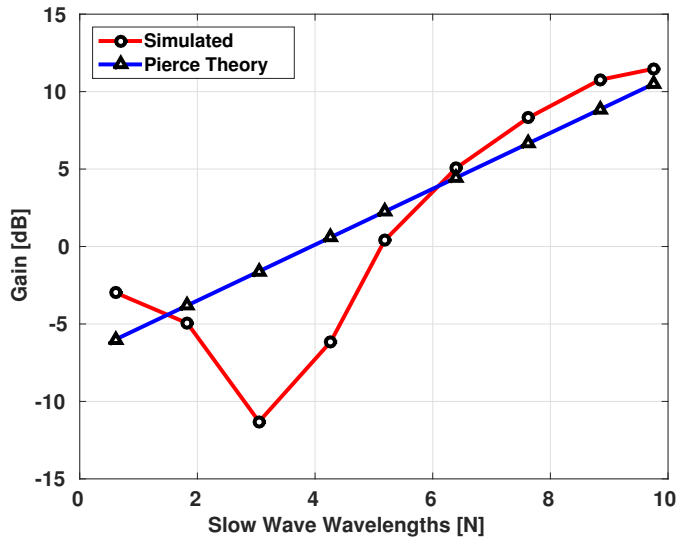

(b)

Figure 7.35: The (a) phase and the (b) gain along the circuit for the square pulse profile with $\phi_{\text {offset }}=0 \mathrm{rad}, L_{e}=30 \mathrm{~cm}, L_{p}=1 \mathrm{~cm}, P_{r f}=1 \mathrm{~W}$, and $I_{\text {beam }}=150 \mathrm{~mA}$. 


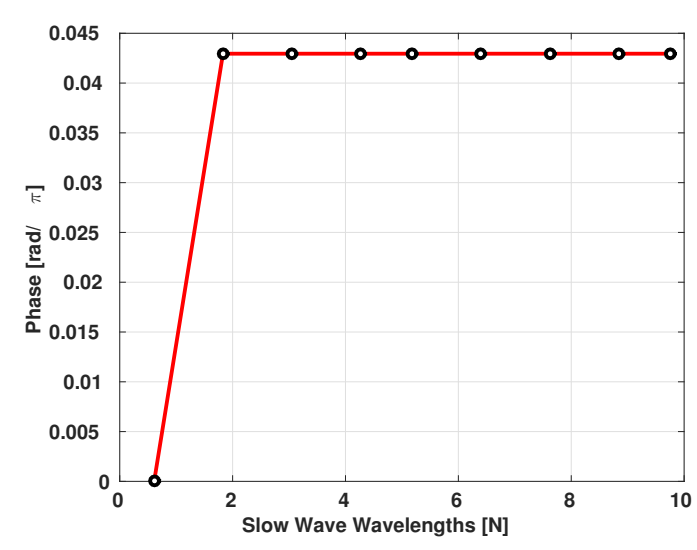

(a)

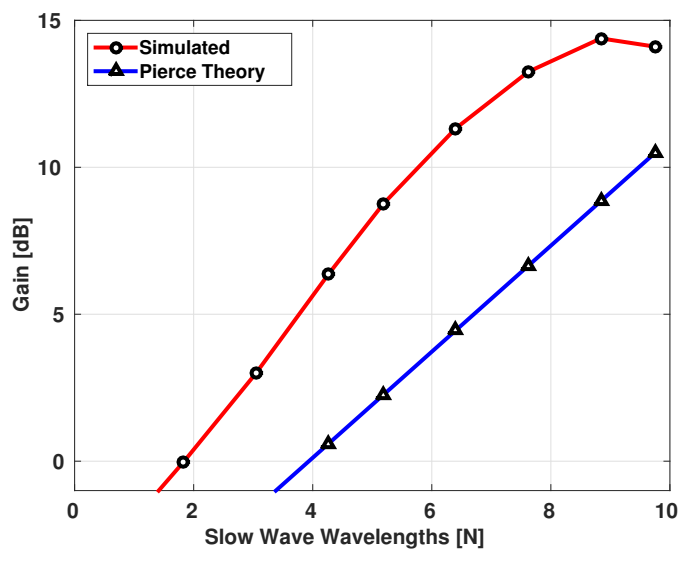

(b)

Figure 7.36: The (a) phase and the (b) gain along the circuit for the square pulse profile with $\phi_{\text {offset }}=\pi \mathrm{rad}, L_{e}=30 \mathrm{~cm}, L_{p}=1 \mathrm{~cm}, P_{r f}=1 \mathrm{~W}$, and $I_{\text {beam }}=150 \mathrm{~mA}$.

\subsubsection{Emitter Length}

Another repeat study is the emitter length study. Figure 7.37 shows the gain and corresponding currents of the emitter length sweep. In this case, the gain increases with emitter length. The current to the cathode/sole region are also much lower than the current observed for the sine wave profile. 


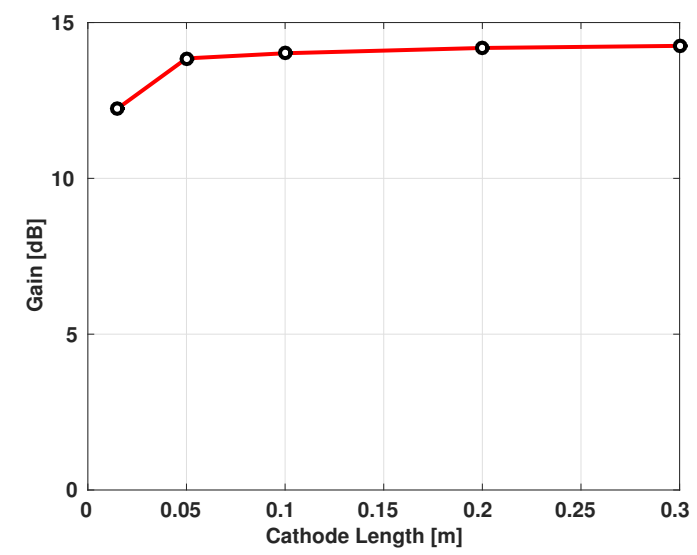

(a)

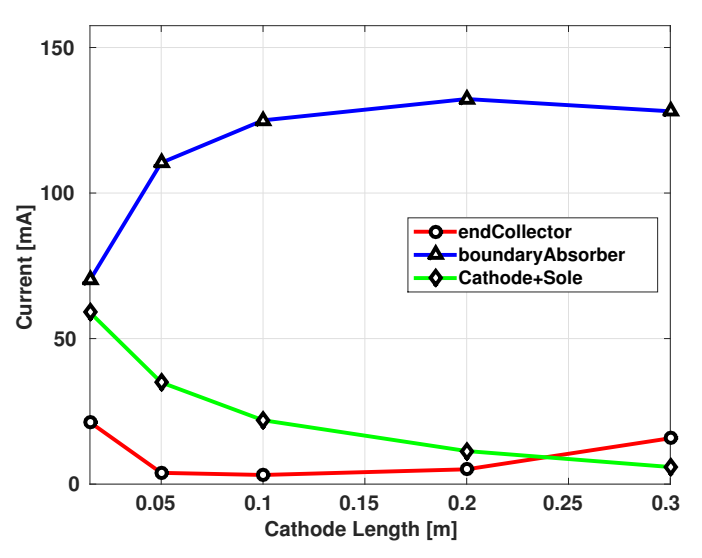

(b)

Figure 7.37: (a) The gain and the corresponding (b) SNR vs. the phase difference between the beam profile and the RF wave for $L_{e}=30 \mathrm{~cm}, P_{r f}=$ $1 \mathrm{~W}$, and $I_{\text {beam }}=150 \mathrm{~mA}$.

\subsubsection{RF Input Power}

The effect of the RF input power on output power for both the square pulse profile and the injected beam is shown in Fig. 7.38. The corresponding SNR and efficiency is shown in Fig. 7.39. Figure 7.38(a) includes plots with a cathode power of both $P_{g f e a}=1 \mathrm{~W}$ and $P_{g f e a}=0.1 \mathrm{~W}$. The gain using $P_{g f e a}=1 \mathrm{~W}$ is shown to demonstrate the predicted gain of the CFA design with the current GFEA cathode technology. The gain using $P_{\text {gfea }}=0.1 \mathrm{~W}$ is shown to demonstrate the gain for a higher power CFA design. With a higher power design, the modulated cathode power is less than 3 orders of magnitude of the output power. Instead of increasing the power of the design, which is much more difficult, the estimated cathode power is lowered. The x-axis shows the RF input power on the circuit with the data below $P_{r f}=10^{-2} \mathrm{~W}$ goes to $P_{r f}=0 \mathrm{~W}$ (This was done to easily show the $0 \mathrm{~W}$ RF input power on the graph). The x-axis does not include the modulated power on the cathode, but the 
calculated gain includes the modulated cathode power.

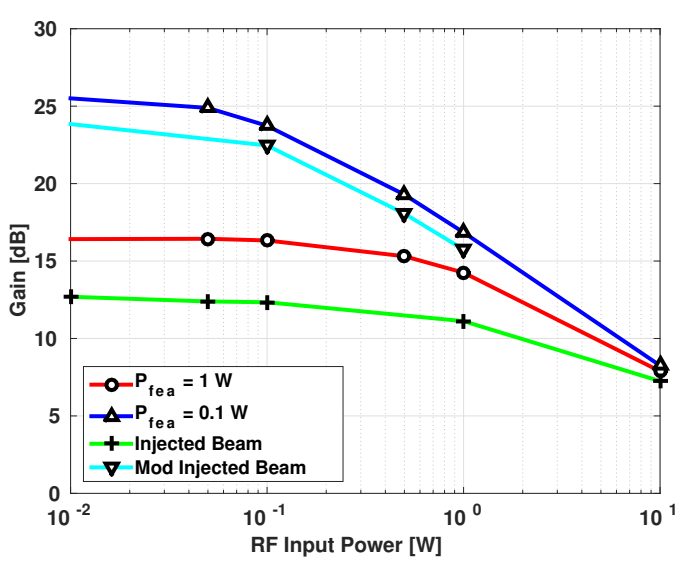

(a)

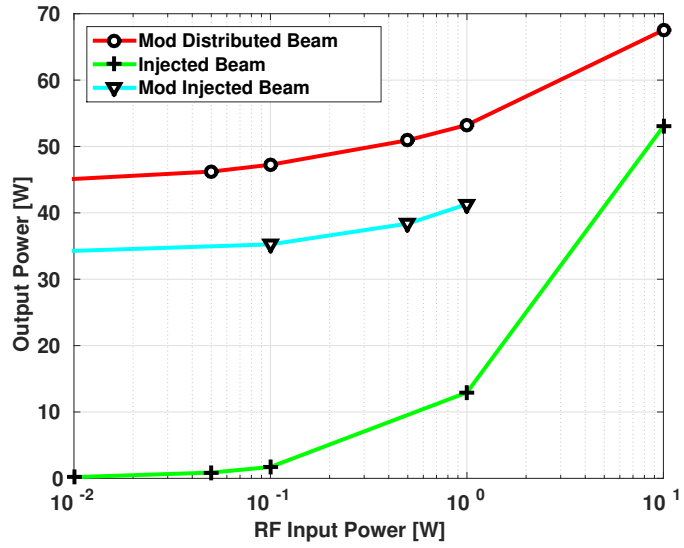

(b)

Figure 7.38: (a) The gain and the corresponding (b) output power vs. the RF input power on the circuit for both the modulated current and the injected beam with $I_{\text {beam }}=150 \mathrm{~mA}$. The modulated cathode uses $L_{e}=30 \mathrm{~cm}$. The RF input power on the $\mathrm{x}$-axis does not include the modulated cathode power. In (a), the red line includes a $1 \mathrm{~W}$ modulated cathode power and the blue line includes a $0.1 \mathrm{~W}$ modulated cathode power. 


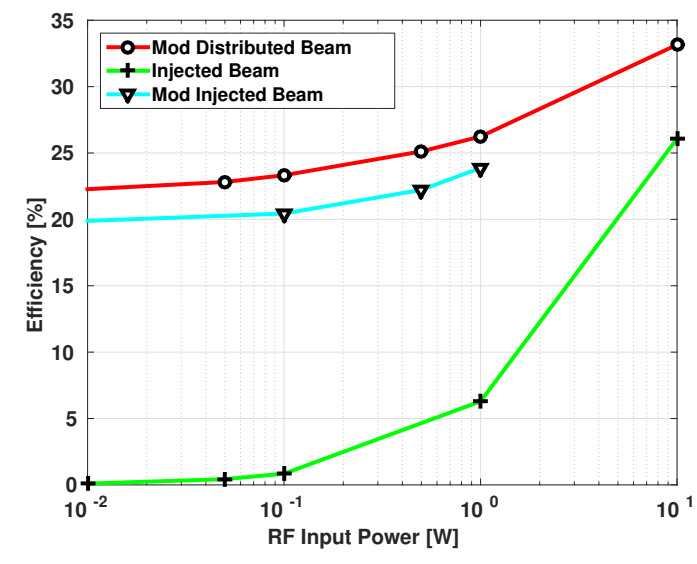

(a)

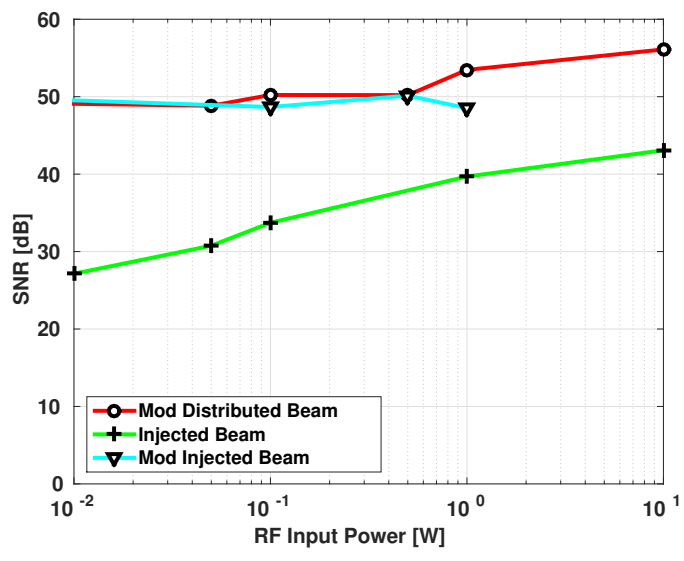

(b)

Figure 7.39: (a) The efficiency and the (b) SNR vs. the RF input power on the circuit for both the modulated cathode in red and the injected beam in green with $I_{\text {beam }}=150 \mathrm{~mA}$. The modulated cathode uses $L_{e}=30 \mathrm{~cm}$. The $\mathrm{RF}$ input power on the $\mathrm{x}$-axis does not include the modulated cathode power.

Note that the RF input power has less effect on the gain and the output power when using a modulated cathode rather than the injected beam cathode. This is because most of the gain is contributed by the modulation of the cathode itself. This brings the effectiveness of using any RF input signal into question. To determine the effectiveness of the RF input signal in contributing to the gain of the device, the contribution of the modulated cathode to the output power is subtracted. The contribution of the modulated cathode is determined from the output power observed when $P_{r f}=0$, which is $43 \mathrm{~W}$. Figure 7.40 shows the contribution to the gain and output power from the RF input power alone for the modulated and injected beam cathode. Note that the injected beam case is not modulated. As the RF input power increases the gain decreases but the total output power increases for both cases. 


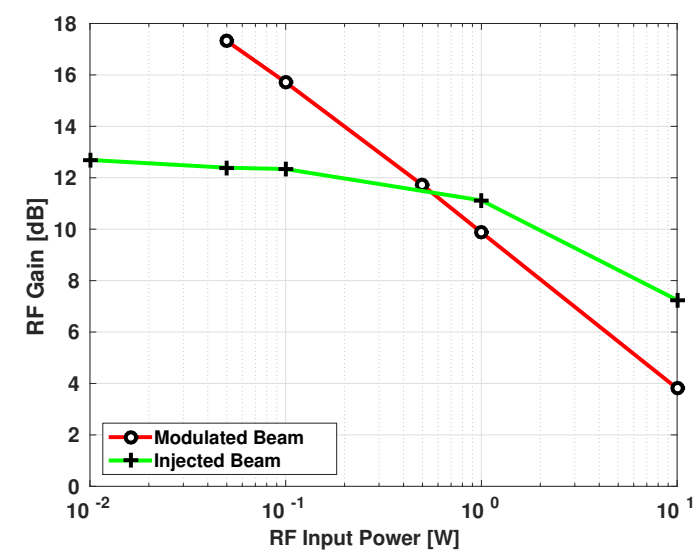

(a)

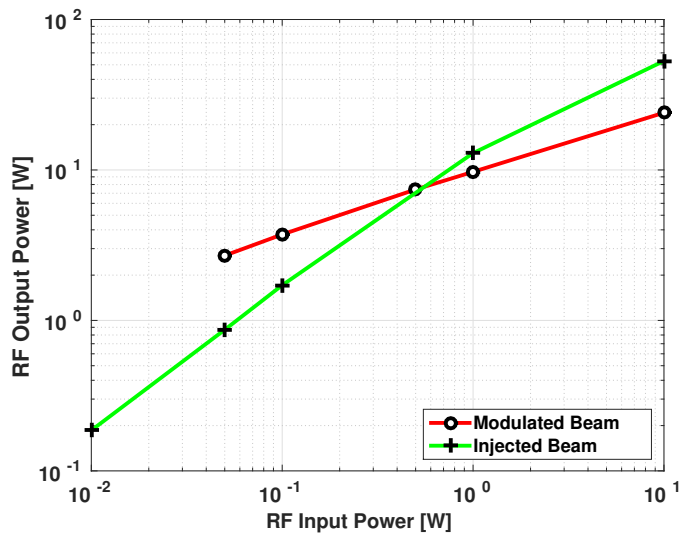

(b)

Figure 7.40: The RF input signal contribution to (a) gain and the corresponding (b) output power vs. the RF input power on the circuit for both the modulated and injected beam cathode. The RF input power on the $\mathrm{x}$-axis does not include the modulated cathode power.

\subsubsection{Beam Power Effects}

The beam current was varied to see the scaling of the output signal with increasing the beam power of the device. Figure 7.41 shows the gain and the output power of both the modulated cathode and the uniform distribution. Once again, two gains are displayed for the modulated cathode case, one with $P_{g f e a}=1 \mathrm{~W}$ in red and the other with $P_{g f e a}=0.1 \mathrm{~W}$ in blue. The modulated cathode uses $L_{e}=30 \mathrm{~cm}$, and the uniform current uses $L_{e}=20 \mathrm{~cm}$. The uniform cathode is as short as possible to maximize gain by injecting the beam as close to the beginning of the circuit as possible while minimizing space charge effects due to the higher current densities. As the current increases, the gain increases for all cases. Note that the output power for the modulated case is much greater than the unmodulated.

The corresponding efficiencies and SNR are shown in Fig. 7.42. The SNR and efficiency for the modulated cathode are both much greater than the unmodulated 
case. The corresponding currents are shown in Fig. 7.43, which help to explain the trends in efficiency and SNR.

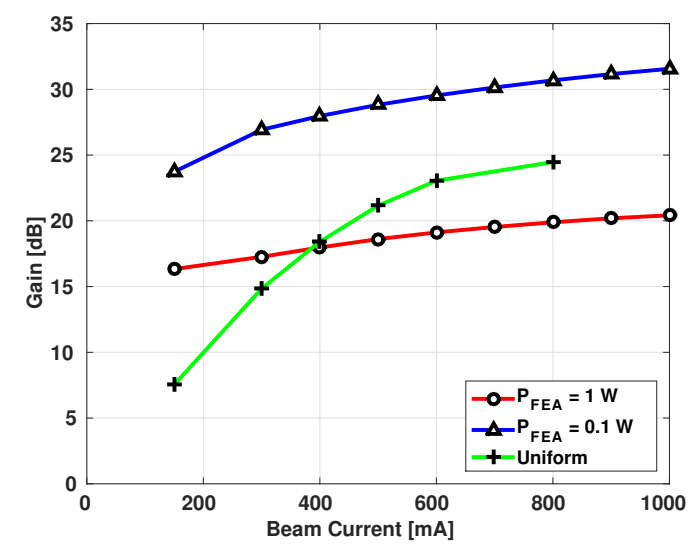

(a)

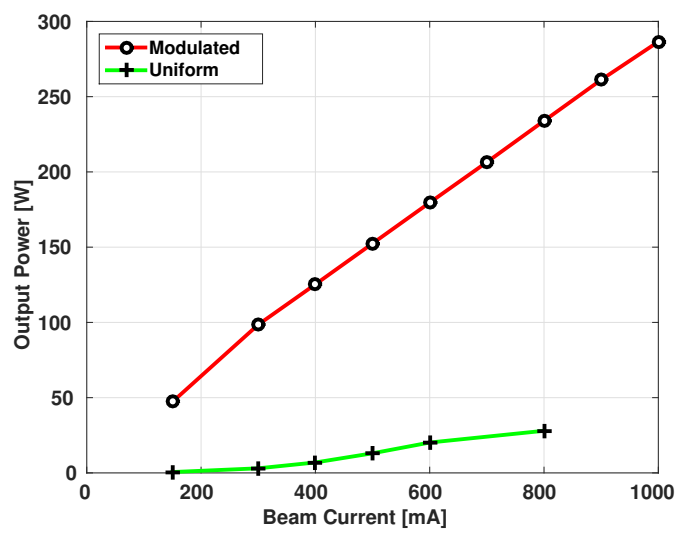

(b)

Figure 7.41: (a) The gain and the corresponding (b) output power vs. the beam current for both the modulated and uniform current distributions with $P_{r f}=0.1 \mathrm{~W}$. In (a), the red line includes a $1 \mathrm{~W}$ modulated cathode power, the blue line includes a $0.1 \mathrm{~W}$ modulated cathode power, and the green line shows the uniform current case. The modulated cathode uses $L_{e}=30 \mathrm{~cm}$ and the uniform current uses $L_{e}=20 \mathrm{~cm}$.

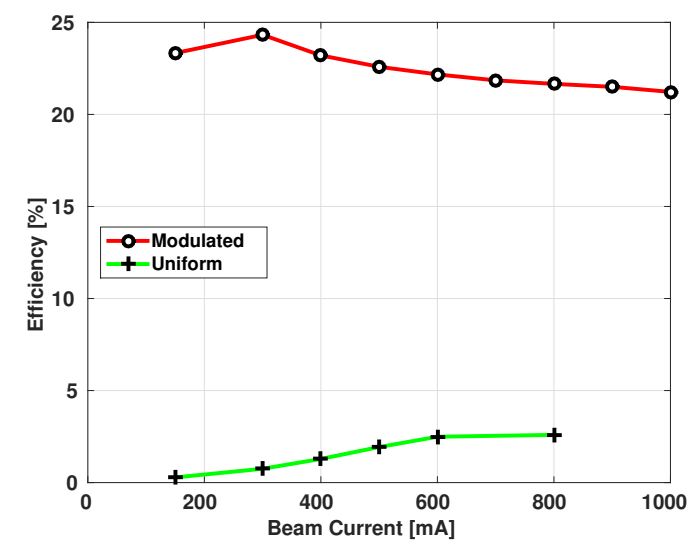

(a)

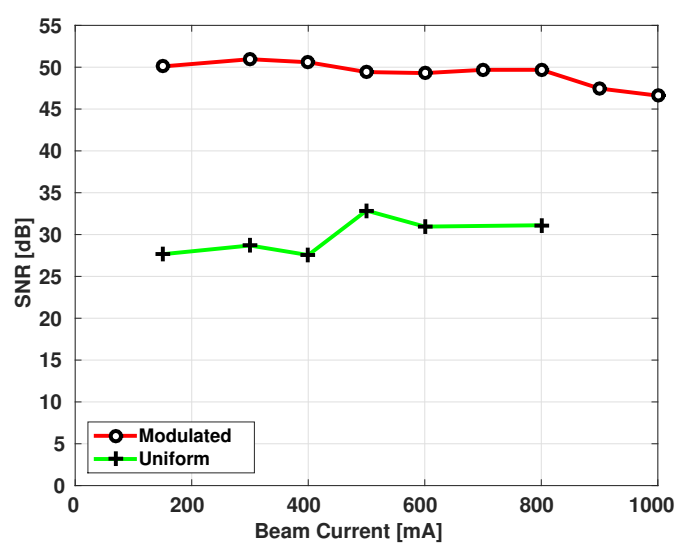

(b)

Figure 7.42: (a) The efficiency and the (b) SNR vs. beam current on the circuit for the modulated distribution in red and the uniform distribution in green. 


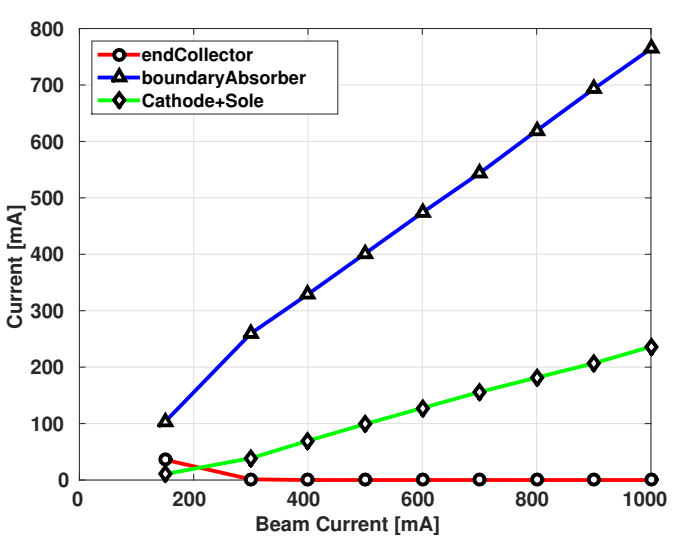

(a)

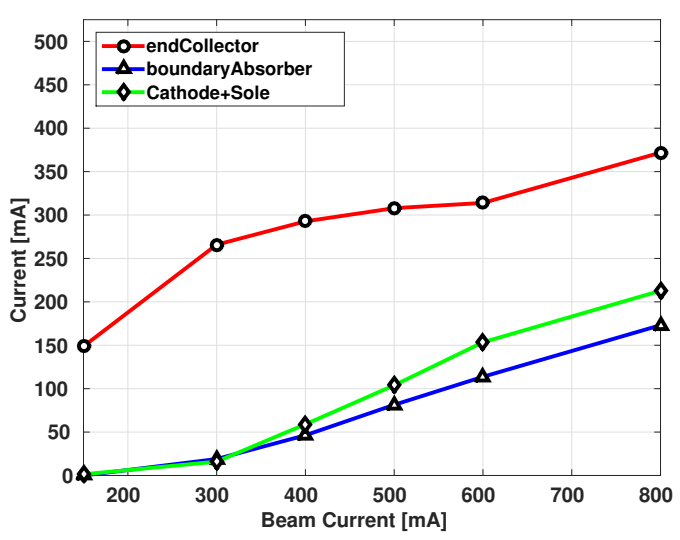

(b)

Figure 7.43: (a) The currents for the modulated cathode compared to (b) the currents of the uniform cathode for the beam current sweep.

\subsubsection{General Effects}

As a visual aid, snapshots of the electron positions for the various cathode configurations is shown. The electron positions at different times for the square wave profile are shown in Fig. 7.44. With this optimum emission profile, cylindrical bunches of electrons are observed. The electron positions for the different beam injection methods at different RF input powers are shown in Fig. 7.45. The unmodulated injected beam trajectories shown in Figs. $7.45(\mathrm{a}, \mathrm{b}, \mathrm{e}, \mathrm{f})$ show little bunching at $P_{r f}=0.5 \mathrm{~mW}$ where the $P_{r f}=10 \mathrm{~W}$ case shows significant bunching with actual cylindrical electron bunches formed in the higher power uniformly distributed beam case. The trajectories for the sine wave modulated injected beam show well defined bunches and show slight variations between the different RF input powers. The higher power modulated distributed beam case shows the electrons approaching the anode as they travel down the tube as they give up energy to the RF wave. The cylindrical bunches also become thinner farther down the tube as electrons are lost to the anode. 


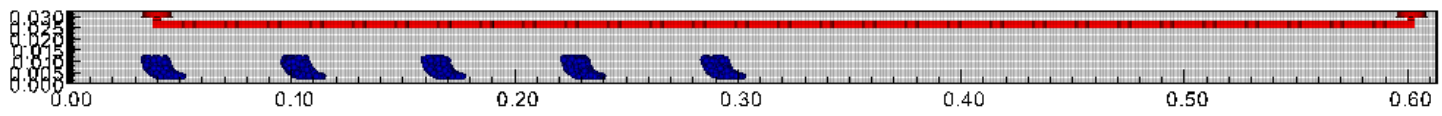

(a) $t=1.36 \mathrm{~ns}$

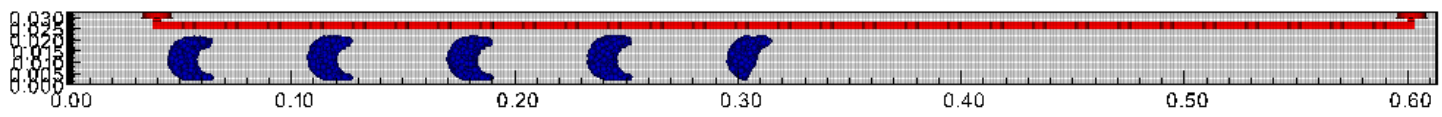

(b) $t=2.62 \mathrm{~ns}$

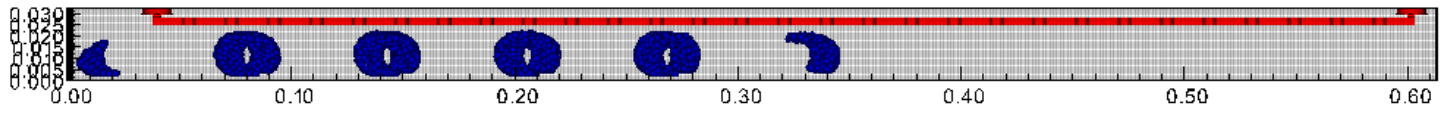

(c) $t=4.86 \mathrm{~ns}$

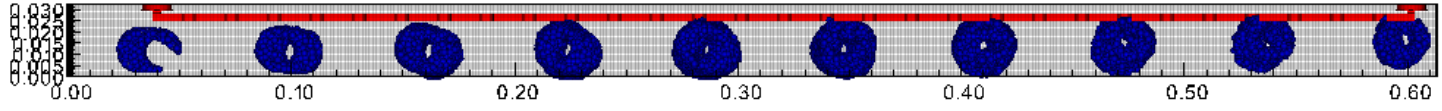

(d) $t=112.9 \mathrm{~ns}$

Figure 7.44: Electron trajectories for the square pulse emission profile for $V_{a s}=1550 \mathrm{~V}$ and $L_{e}=1 \mathrm{~cm}$ as time progresses. 


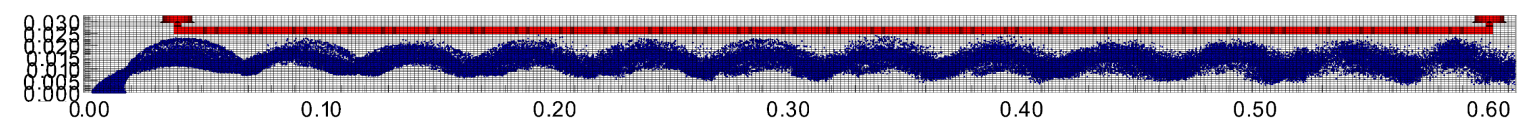

(a) Injected Beam, $P_{r f}=0.5 \mathrm{~mW}$

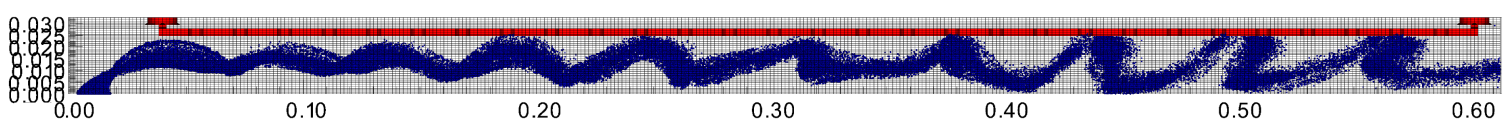

(b) Injected Beam, $P_{r f}=10.0 \mathrm{~W}$

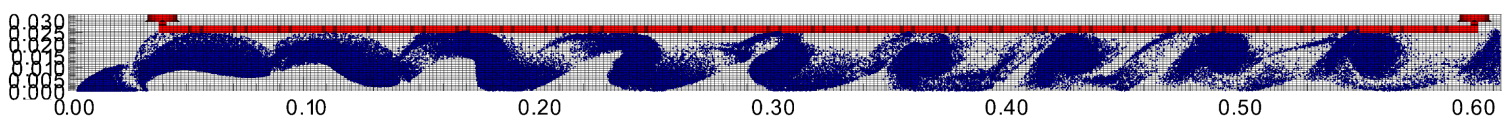

(c) Sine Wave Modulated Injected Beam, $P_{r f}=0 \mathrm{~W}$

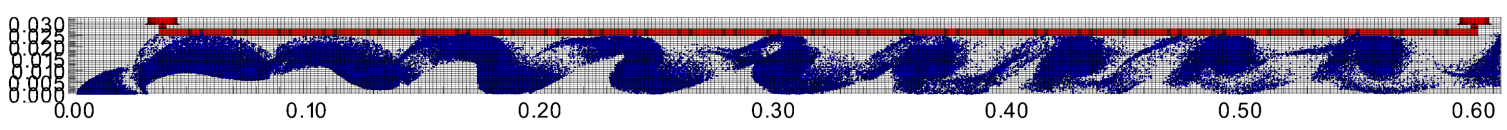

(d) Sine Wave Modulated Injected Beam, $P_{r f}=1.0 \mathrm{~W}$

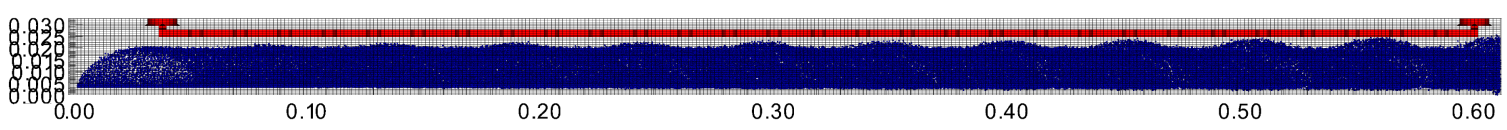

(e) Uniformly Distributed Beam, $L_{e}=10 \mathrm{~cm}, P_{r f}=0.5 \mathrm{~mW}$

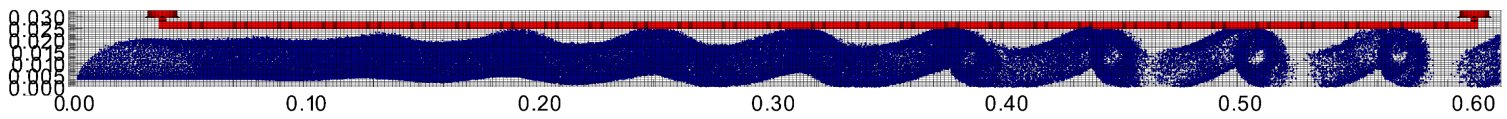

(f) Uniformly Distributed Beam, $L_{e}=10 \mathrm{~cm}, P_{r f}=10 \mathrm{~W}$

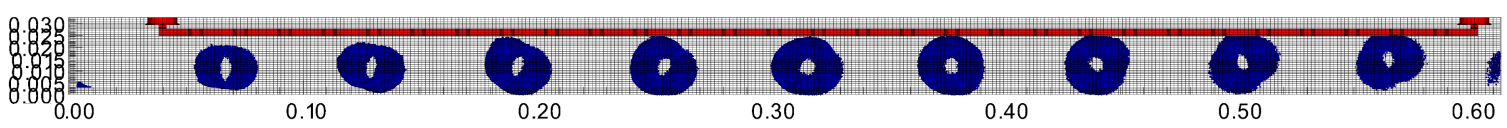

(g) Modulated Distributed Beam, $L_{p}=1 \mathrm{~cm}, L_{e}=10 \mathrm{~cm}, P_{r f}=0 \mathrm{~W}$

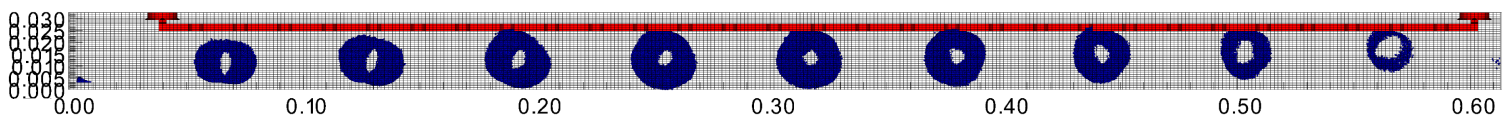

(h) Modulated Distributed Beam, $L_{p}=1 \mathrm{~cm}, L_{e}=10 \mathrm{~cm}, P_{r f}=10 \mathrm{~W}$

Figure 7.45: Electron trajectories for various beam injection types and RF input powers with $V_{a s}=1550 \mathrm{~V}$ and $I_{\text {beam }}=150 \mathrm{~mA}$. 


\subsubsection{Discussion}

This section is broken into two parts. The first part discusses the general characteristics of the modulated cathode itself. The next section compares all the beam injection methods.

\subsubsection{Modulated Cathode Characteristics}

The maximum gain in Fig. 7.32(a) and 7.34(a) occurs when the peak electron current aligns with the decelerating RF electric field, $\phi_{\text {offset }}=\pi$. This is exactly what theory suggests, the decelerating field extracts energy from the beam causing amplification of the RF wave. An interesting result is that the minimum gain is not less than $0 \mathrm{~dB}$. If the majority of electrons are in the accelerating fields, the RF wave gives up its energy to the beam and should cause amplification of less than $0 \mathrm{~dB}$.

The cause of the moderate gain of unsynchronized beam profiles is due to the modulating signal overpowering and driving the RF wave on the circuit. Fig. 7.35(a) shows how the phase on the circuit shifts to match that of the emission profile phase. The gain along the circuit, shown in Fig. 7.35(b), shows the gain decreasing as the phase is shifting, and then once the decelerating fields of the RF wave on the circuit is aligned with the emission profile peaks, the gain starts to increase. When the phases are intentionally aligned, Fig. 7.36(a) shows almost no phase shift of the RF wave, and the gain immediately starts increasing as shown in 7.36(b).

The gain along the circuit shown in Figs. 7.35(a) and 7.36(a) does not follow the Pierce theory gain line. This is to be expected as the beam assumption used to develop the Pierce theory is no longer valid due to the pre-bunched beam. With the pre-bunched beam, if the phase alignment is optimum, the gain immediately starts 
increasing rather than the gradual gain predicted by Pierce theory with the $-6 \mathrm{~dB}$ initial condition. Also, the gain increases at a greater rate in the mid-region of the circuit. This increase in gain is because the beam is pre-bunched and is less limited by the beam impedance.

When using the modulated cathode, the effect of the RF power input on the circuit is found to have less impact on the output power than the effect of the modulated cathode. As shown in Fig. 7.38(b), with no RF input power the modulated distributed cathode output power is $43 \mathrm{~W}$ and the relative increase to the output power from changes in the input $\mathrm{RF}$ are rather small. The contribution of the RF input power on the circuit to the output power is shown in Fig. 7.40(b) and is compared to the injected beam. This plot shows that adding a RF input on the circuit does contribute to the gain, but becomes less effective at higher input powers. For RF input powers less than $0.5 \mathrm{~W}$, the contribution of the circuit RF input power on the output power is actually greater than the injected beam. At RF input powers greater than $0.5 \mathrm{~W}$ contribution to the output power is is less effective than the injected beam. This result shows that adding an RF input power on the circuit along with the modulated cathode is effective and improves the efficiency of the device with little to no cost to gain at lower input powers. However the contribution to the RF output power is $<2 \%$ of the total output power at the lower input powers which only improves the efficiency by a few percent and this small efficiency improvement may not be enough to overcome the cost of the complexity of implementing the timing between the RF signal on the circuit and the modulated cathode. But this small improvement on this low power device might scale well with a higher power device. This still needs to be determined.

The effect of the emitter length when using the modulated cathode shows con- 
flicting results. For the sine wave profile, Fig. 7.33(a) shows the gain decreasing as the circuit gets longer after $L_{e}=5 \mathrm{~cm}$. The increase in gain from $1.5 \mathrm{~cm}$ to $5 \mathrm{~cm}$ is because of better beam injection optics due to reduced space charge. The decrease in gain after $L_{e}=5 \mathrm{~cm}$ is because of the lower effectiveness of electrons injected farther down the tube. This result corroborates with the results found in Sec. 7.6.1.2. Figure 7.37(a) shows a sharp increase in gain from $1.5 \mathrm{~cm}$ to $5 \mathrm{~cm}$, which is consistent with the other results, but also shows a gradual increase at emitter lengths greater than $5 \mathrm{~cm}$, which is inconsistent. The continued increase after $5 \mathrm{~cm}$ is caused by beam optics. All previous results showed the sensitivity of the gain to the beam injection optics, and the modulated distributed cathode is no different. As the emitter length is increased, the peak input current density of the pulse decreases, which alters the beam trajectory which has a significant effect on the beam interaction with the RF wave on the circuit.

The cylindrical bunches observed in Fig. 7.44 is caused by the varying velocities of a cycloiding electron. Each point of emission travels at the $\mathbf{E} \times \mathbf{B}$ velocity in the $x$-direction, the velocity of the electrons in the interaction region varies as it travels in the $x$-direction; but with the average $\mathbf{E} \times \mathbf{B}$ velocity. The initial velocity after being emitted is close to zero. The electrons accelerate in the $y$-direction because of the static electric field. The magnetic field causes a tangential force on the moving electron, eventually shifting the velocity in the $x$-direction where the maximum $x$ velocity occurs at the top of the cycloid. The continued tangential magnetic force pushes the electron back towards the cathode, where the total velocity is zero right near the cathode. This changing velocity causes the cylinder bunches in the electron beam. 


\subsubsection{Beam Injection Method Comparison}

The gain, output power, SNR, and efficiency when using a modulated cathode are much greater than using an unmodulated cathode. The RF input power sweep shown in Figs. 7.38 and 7.39 show both the modulated distributed and modulated injected cathodes having more gain, output power, SNR, and efficiency than the unmodulated injected beam, even when accounting for a higher cathode power. The small-signal-gains observed for the modulated cathodes greatly outperform the unmodulated cathode, although the quantity of the improvement is questioned by the estimated GFEA drive power. The CFA using the unmodulated cathode extracts energy from the beam by modulating the beam through the interaction between the $\mathrm{RF}$ wave alone. At lower RF input powers, the electric fields in the interaction region are smaller, which reduces the energy extraction rate from the electron beam, which doesn't lower the gain, but lowers the output power of the device. The output energy of the CFA using the modulated cathode comes mainly from the modulated cathode itself. The pre-bunched beam drives the RF wave on the circuit, and any RF input signal modulation of the beam is a secondary gain mechanism, so the output power remains high even with low RF input powers on the circuit. With higher RF input powers on the circuit, all the methods tend to converge to the same output power as the amplifier saturates. Estimated by the highest efficiency observed in Fig. 7.39, the maximum efficiency of the device seems to be around $35-40 \%$, mainly limited by the highly cycloidal beam as discussed in Sec. 7.4.3.3. The electron beam becomes depleted as electrons collect on the slow wave circuit, and the gain saturates. The energy extraction rate of the RF wave interaction with the beam becomes more

efficient as the RF input power is increased. As both the modulated and unmodulated 
cathode configurations approach the maximum efficiency, the output powers of the methods converge.

Figures 7.38 and 7.39 also show the usefulness of the modulated injected beam. The modulated injected beam outperforms the unmodulated injected beam but under performs the modulated distributed beam. Generally, having more current injected towards the beginning of the tube is an advantage, so the modulated injected beam should display more gain than the modulated distributed gain, but in this case, space charge is unfavorable and undermines any advantage gained from injecting all the current at the beginning of the tube. With proper beam injection optics, the characteristics of the modulated injected beam configuration should improve.

The modulated and unmodulated distributed cathode both respond well to increases in beam current. An increase in electron beam current decreases the beam impedance, which increases the gain and efficiency of the device according to Pierce theory as discussed in Sec. 2.5.3. The gain and efficiency for the uniform profile greatly benefits from an increase in beam current, shown in Figs. 7.41 and 7.42. The gain of the modulated cathode also improves with increased beam current, but the efficiency actually decreases from $24 \%$ to $21 \%$, shown in Fig. 7.42. The decreased efficiency is due to space charge altering the electron trajectories and spreading the electron bunches. The larger current increases the gain, but the spreading of the electron bunches decreases efficiency faster than any efficiency improvement due to the inherent increase in beam current.

The gain of the unmodulated cathode does surpass the modulated cathode when using a predicted cathode power of $P_{F E A}=1 \mathrm{~W}$ at $I_{\text {beam }}=400 \mathrm{~mA}$ in Fig. 7.41. This shows the unmodulated cathode outperforming the CFA design using SW3 as it is, but by approximating a higher power device by estimating a smaller RF drive power, 
the gain is significantly greater at all beam currents. The gain at $I_{\text {beam }}=800 \mathrm{~mA}$ exceeds $30 \mathrm{~dB}$ for the modulated case, where the unmodulated only shows a $25 \mathrm{~dB}$ gain. This result shows two main concepts. The first concept reiterates that the small signal gains are improved when using the modulated cathode, but GFEA drive power reduces the benefits on lower power devices. The second concept is that higher beam currents tend to improve the uniform current model more than the modulated current. It is possible that with higher beam currents, the gain of the methods may converge as they did with higher RF drive powers.

\subsubsection{Summary}

A modulated distributed and injected beam cathode was studied and compared with unmodulated cathode types and was shown to improve gain, efficiency, SNR, and output power. In the unmodulated case, electron beam bunching process was achieved by the RF input alone, which is limited by the interaction impedance and the RF input power. With the modulated cathode, the ideal electron beam bunching is performed mainly by the cathode, and the strong electric fields created by these pre-formed bunches drive the RF wave on the circuit. Without any RF input on the circuit, substantial output power was observed using the modulated cathode. The RF input on the circuit was found to enhance the beam energy extraction if the emission profile peaks were aligned with the decelerating portions of the RF wave on the circuit, but only by $<2 \%$. Different cathode lengths were tested, and it was determined, as was determined in the previous static configurations, that electrons emitted at the start of the tube are more effective than electrons emitted farther down as long as the injection optics are optimized. This result shows that a modulated injected beam configuration is a viable method to implement a highly efficient CFA; however the 
output power would be limited by the ability to inject high currents optimally. To improve higher power devices, a distributed cathode would be necessary to achieve the necessary currents required.

The CFA used in this work is a very low power design, and the amount of GFEA modulation benefit to small-signal-gain is questionable due to the uncertainty in the required RF drive power of the GFEA. For this low power CFA, using current GFEA technology, the RF drive power was estimated to be about $1 \%$ of the total possible output power. This limits the the lowest possible "small-signal," to $1 \%$ of the output power which is not exactly a small signal in this CFA configuration. For higher power devices, the RF drive signal is $<0.1 \%$ of the total RF output power, and to estimate the small-signal-gain in a higher power device, the estimated GFEA drive power was lowered. Using this lower estimate, the small-signal-gains of the modulated cathode were much greater than the unmodulated case. The RF output power, efficiency, and SNR were always found to be superior to the unmodulated case; however at higher RF input powers, the gain, output power, and efficiency were comparable.

Higher RF input power improves the efficiency of all devices. This is why the characteristics of each methods match. The benefits of the modulated cathode over the unmodulated one become less apparent. Higher RF drive signals can efficiently form electron beam bunches and extract energy from the beam. This region questions whether the use of the modulated cathode over the unmodulated design actually is an improvement. The results show that the gain, output power, and efficiency are better, but not by much, and the improvement is expected to decline with even higher RF powers. Also, higher beam powers were shown to benefit the the unmodulated cathode more than the modulated one. This result further reduces the expected improvements of the modulated cathode. The biggest benefit of the modulated cathode at higher RF 
input powers is the SNR. The SNR when using the modulated cathode is $\sim 50 \mathrm{~dB}$ whereas the SNR for the unmodulated cathode is $\sim 30 \mathrm{~dB}$, a $20 \mathrm{~dB}$ improvement. Not only are there the inherent advantages of the low SNR itself, but this is very important because this may allow for high gain and high power CFAs, along with the low noise.

Current high power CFAs have relatively low gain $(<20 \mathrm{~dB})$ because of the necessity of a high RF drive power to retain lock on the main amplifying mode. Part of this limit is due to noise, and modulating the cathode may allow for even higher RF output powers and high gain. To find the maximum achievable output power of the device, the electron beam and RF drive power should be increased. The maximum achievable output power using all of the cathode configurations for the NU CFA was not determined because of time constraints. The most common limit to gain and output power observed was due to limitations in beam current from space charge and not due to the mode locking limit. With proper design, this space charge limit can be overcome, and then the true limit due to mode locking can be studied.

\subsection{Implementation on Higher Power CFA Designs}

The use of GFEAs in low power CFAs shows some improvement, but the margin is small when compared to the unknown increase in GFEA drive power. By using GFEAs in higher power devices, because of the exponential relationship between gate-to-emitter voltage and output current, the GFEA drive power is relatively much less than the output power. Using GFEAs in a high power CFA is an attractive prospect, but there are many potential issues.

This section outlines a direct application of the modulated distributed cathode 
strategy on a higher power device. Using the CFA design by Eppley et. al. [83], described in Sec. 2.8.1.2, some theoretical predictions and potential issues are given. For high power devices such as this, the use of GFEA in the manner in which they were used in this work will be very difficult due to the high current densities and high electric fields in the interaction region. Some other possible methods to implement GFEAs in CFAs are proposed.

\subsubsection{Direct Implementation}

Here the modulated distributed cathode is applied to the CFA design by Eppley et. al. [83] described in Sec. 2.8.1.2. The application of the GFEA on this device is realistically questionable, but ignoring the device implementation, the best case scenario is presented here. The emitted current density in that work was $41 \mathrm{~A} / \mathrm{cm}^{2}$ uniformly distributed across the cold cathode with a total of $2600 \mathrm{~A}$ observed at the anode. To achieve a meaningful amount of modulation using the square pulse emission profile, the peak current density will be $82 \mathrm{~A} / \mathrm{cm}^{2}$ from a half slow wave wavelength pulse length $\left(L_{P}=\lambda_{s w} / 2\right)$. By using the procedure to calculate drive power outlined in Sec. 5.3.7.4, the emitter-to-gate voltage is modulated from $V_{g e}=30 \mathrm{~V}$ to $V_{g e}=$ 70 Vat $f=11.424 \mathrm{GHz}$, which estimates the RF drive power at $P_{g f e a}=10 \mathrm{~W}$ at the low end using Eq. (5.8), and $P_{g f e a}=500 \mathrm{~W}$ at the high end using Eq. (5.6).

Conceivably, the observed full output power of the original device will also be observed when using the GFEA setup, if not more. Using the upper estimate of RF drive power and no RF circuit drive power, this would suggest a gain of $58 \mathrm{~dB}$. This is incredible but this is most likely unrealistic. In order to implement a distributed cathode, something must be used to control the emission energy of the electrons and protect the GFEA from damage from the interaction region. Hop funnels are 
proposed here, which demonstrates both qualities, but at these high current densities, introduces its own problems. Essentially, electrons are emitted directly into the dielectric of the hop funnel which causes a high amount of heat, causing structural failure, and the potential to knock ions off the surface, causing damage to the cathode from ion back bombardment [24] onto the GFEA. The heating of the dielectric can be solved by careful cooling design, but the performance of the hop funnels at high current densities is questionable.

Another direct application design idea is to use a shielded cathode concept [13, 112], which uses lateral emitters with carefully designed optics to inject electrons at the correct energy through slits in the sole electrode. Figure 7.46 shows a diagram of this beam injection method [112]. Electrons are emitted by gated lateral field emitters and are pushed up through the slit in the sole electrode by the pusher electrode. Electron energy is controlled by the emitter tips and the gate and is independent of the sole electrode potential. The emitter tips are tucked away behind the sole electrode, well protected from ion or electron bombardment from the interaction region. This work showed the viability of this concept via simulation, but has not been implemented experimentally. This method meets all the requirements for direct application and is a very promising method, but the implementation is difficult, requires much more work, and may prove to be unreliable. 


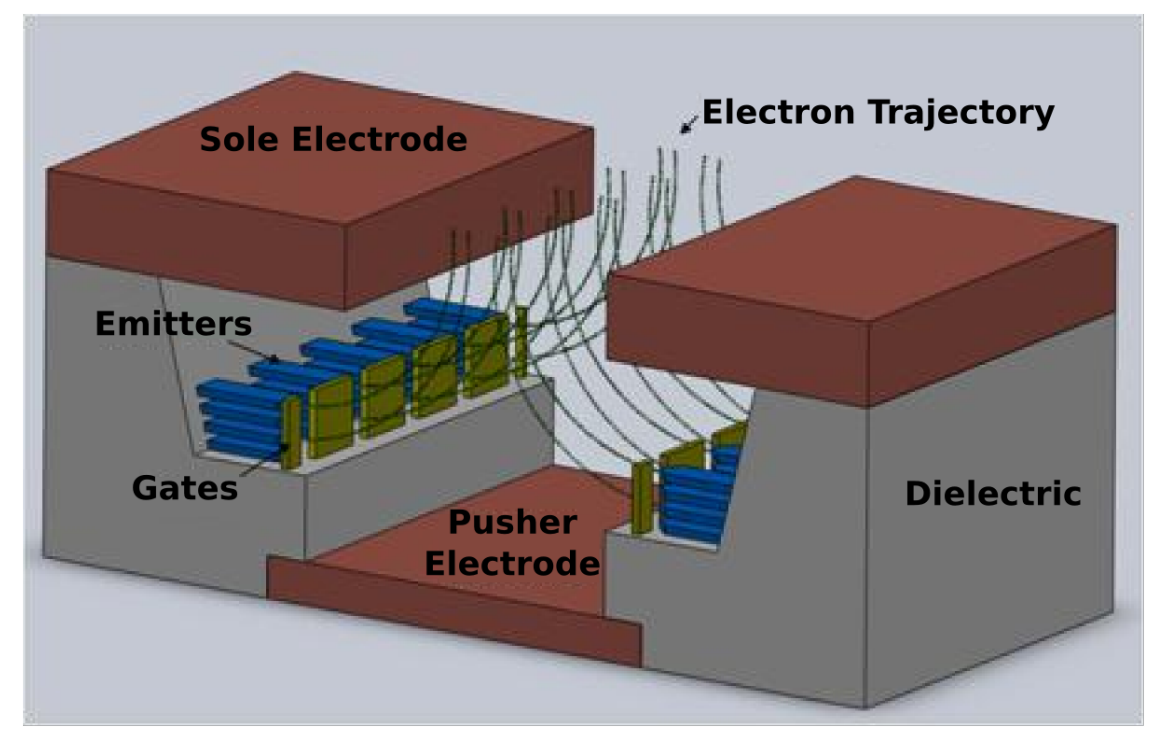

Figure 7.46: Diagram of the shielded cathode slit concept. Lateral gated field emitters on each side of the slit emit electrons and are pushed out through the slit in the sole electrode by the pusher electrode [112].

\subsubsection{Alternate Implementations}

The direct application using GFEAs as a distributed cathode is maybe unfeasible at this point, so a few alternate implementations are suggested here. These ideas suggest a hybrid option using GFEAs in conjunction with alternate cathode options. This way the majority of the current can be supplied by commonly used cathodes, and the modulated GFEAs can used to control the alternate cathode current, as in the secondary emitting implementation, or supplement it as to provide a mode locking mechanism to allow for higher power.

\subsubsection{End Hat Injection}

To keep the GFEAs relatively protected from the high electric fields and electron currents in the interaction region, the GFEAs could be placed near or on the end 
hats. Figure 7.47 shows a diagram of this implementation. The current can be supplied all by the GFEAs at the end hats or by a combination of a traditional cathode (thermionic or secondary emitting) and the end hat GFEA. The traditional cathode supplies the majority of the current while the end hat emitters add modulated electron current to promote a mode locking mechanism to possibly improve gain at higher powers. It still has to be shown that partial modulated current will improve CFA performance.

\section{End hat}

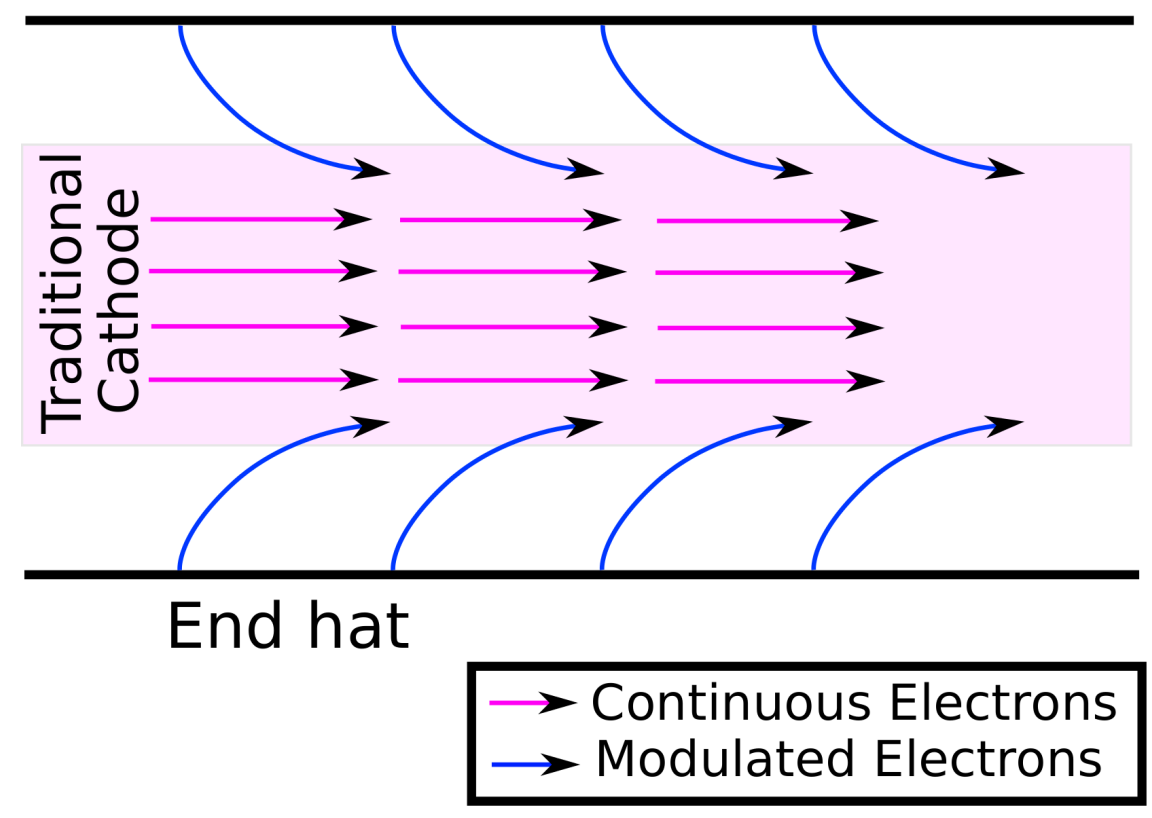

Figure 7.47: Diagram of a CFA using end hat assisted injection. The majority of the electron current is supplied by a traditional thermionic or secondary emitting cathode (violet arrows), and modulated electrons are injected in at the end hats (blue arrows).

\subsubsection{Distributed GFEA Assisted Secondary Emission}

Distributed secondary emitting cathodes (commonly referred to as the emitting sole) are the most common cathodes in use today for high power devices and can generate 
enormous amounts of current $\left(>50 \mathrm{~A} / \mathrm{cm}^{2}\right)$ reliably for extended periods of time. The limit to the gain in these high power applications is due to the necessity of higher RF drive power to for mode locking. GFEAs can be used in addition to the primary secondary emitting cathode to inject current in phase with the RF wave to promote the desired mode of operation and increase the efficiency of operation. The end hat injection method can be used for this, but a direct approach can also be used. Also, the energies of the electrons emitted from the GFEAs can be designed to collide with the secondary emitting cathode at the optimum energy to maximize the secondary electron yield as to 'multiply' the in phase current.

Figure 7.48 shows this current multiplication concept. Electrons are emitted in phase with the RF wave from GFEA sections with the optimum energy to cycloid back into the secondary emitting cathode to produce the maximum number of secondary electrons (in this case 3 secondary electrons). The secondary electrons cycloid down the tube, and because they are in phase with the decelerating field of the RF wave, they give up energy and cycloid towards the slow wave circuit. Note that the secondary electrons do not re-strike the cathode as in the example shown in Fig. 2.18 in Sec. 2.6.2.2. In that case, the secondary electrons were out of phase with the RF wave, which allowed them to strike the cathode again causing each electron and their offspring to produce more and more electrons. In this case the primary electrons are emitted in phase with the RF so only one electron multiplication occurs. 


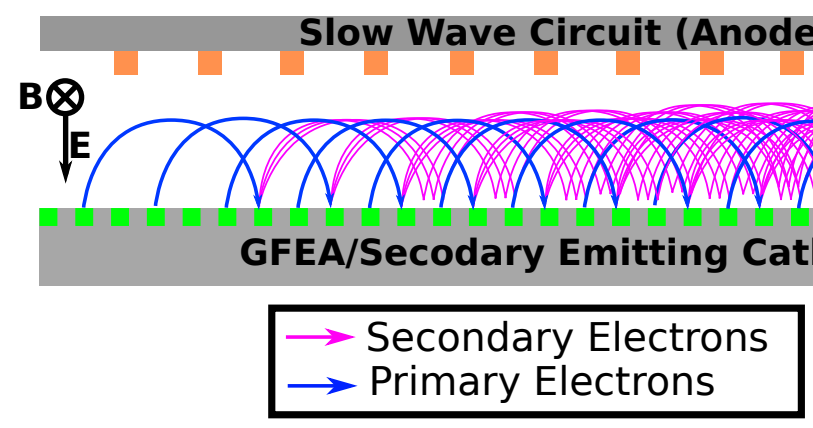

Figure 7.48: Diagram of the GFEA assisted secondary emitting cathode. GFEA locations (these could be hop funnel or the shielded cathode slits) emit electrons in phase with the RF wave with energies so that they collide with the secondary emitting sole at the optimum energy $\left(E_{\max }\right)$ to emit the maximum amount of secondary electrons $\left(\delta_{\max }\right)$.

Figure 7.48 shows operation where all the current is generated from primary electrons from the GFEA. This is one way where all of the electron current is injected in phase with the RF wave. The advantage to using the GFEAs in conjunction with the secondary emitting sole is the initial multiplication of electrons. This lowers the necessity of emission of high current densities from the GFEAs alone. Operating the GFEAs at lower current densities increases the lifetime of the emitters and allows for an easier implementation. A cathode material with a high secondary electron yield would greatly benefit this implementation. Current cold cathode technology uses materials with a $\delta_{\max }<3$, which does not alleviate much of the GFEA current requirements, but is an improvement. It is possible, however, that this new approach will allow use of materials with higher $\delta_{\max }$ discarded for use in conventional CFAs. See Sec. 2.6.2.2 for more information on commonly used materials.

\subsubsection{Injected GFEA Assisted Secondary Emission}

This idea is very similar to the distributed GFEA assisted secondary emission approach, but instead of a number of distributed GFEA emission points, only one 
GFEA emission point is used at the beginning of the tube. This method alleviates the need for shielded cathode ideas or end hat injection. This is a much simpler approach, but can only be implemented in a non-reentrant device. A reentrant device would reintroduce the requirement for a shielded cathode. Most of the current in this device is supplied by the conventional secondary emitting cathode operation, but the modulated injected beam would supplement the current and help provide the mode-locking mechanism. The disadvantage of this method is the lower efficiency due to the non-reentrant CFA design. 


\section{CHAPTER 8}

\section{CONCLUSION}

Using experimental data gathered at BSU and at NU, the VSim simulation CFA model was validated. Also, the VSim simulation results were compared with COMSOL and SIMION simulation results for the circuit dispersion and the electron trajectories, respectively. These results are summarized here. Finally, the distributed, modulated cathode results are discussed.

\subsection{Model Verification}

The first phase of the research to confirm the validity of the VSim model was achieved. Two different CFA devices were studied: a CFA designed at BSU and a device developed at NU. These experimental results were compared against the simulation results.

Dispersion measurements performed here at BSU on a CFA variant which uses a slow wave circuit called SW2 corroborated the dispersion simulated by VSim. The phase velocity differed due to small dimensional differences from experiment and simulation, but the general trends were all confirmed. Full experimental CFA operation of this device showed no gain, and this result was confirmed by the VSim simulation. The simulation showed that a beam current of $150 \mathrm{~mA}$ was needed for 
gain while only $5 \mathrm{~mA}$ was achievable with the PixTech cathodes. Because of the poor performance of this CFA, the research effort was shifted to the design used at NU.

Gain and bandwidth measurements performed at NU on the NU CFA variant matched VSim simulation results. The experimental gain was $5.0 \mathrm{~dB}$ and the simulated gain was $4.0 \mathrm{~dB}$. The slightly lower gain observed overall in the simulation was due to the poor electron injection approximation used in the simulation. Also due to the beam injection, space charge effects in the simulation were found to affect the gain more than in the experiment. The general trends of the simulation and experiment, however, were all confirmed. Also, gain produced by the simulation model was compared and confirmed against the analytic gain predicted by Pierce theory. The simulated bandwidth was slightly smaller than the experimental one. The connectors used in the experimental are believed to be the limiting factor on the bandwidth, and the simulated model used ideal ports, thus increasing the simulated bandwidth slightly. These results show the validity of the model to recreate experimental results, and the model was then altered to implement a distributed cathode.

In order to implement a distributed cathode, electrons must emit from the sole electrode but also be born at a potential less negative than the sole so that they do not immediately collect back on the sole on the next cycloid. A new viable method, which uses a divergence-free region, to simulate a distributed cathode which emits electrons at a less negative potential than the sole potential was developed and studied. This approximation was compared to simulations without the region and was shown to have little impact on the gain at electron currents well below the space-charge limit. This method allows for a simple and low numerical cost implementation of the distributed cathode. 


\subsection{General Observations}

There are a few notable observations about the physics of the CFA operation. The meandering microstrip slow wave circuit has interesting dispersion characteristics worth mentioning. First, the dispersion is found to be rather large, which limits the maximum possible bandwidth of CFA operation. Also, a backward wave mode is observed on the edges of the circuit when the slow wave wavelength is $\lambda>2 P$. This

backward wave mode, without proper techniques to suppress oscillations, imposes another limit to the bandwidth of the CFA operation. Also, small variations in slow wave circuit geometry can have a rather large effect on the predicted phase velocity. Comparisons between simulation and experiment showed rather large differences in phase velocity for the higher frequency $(600-1000 \mathrm{MHz})$ slow wave circuits due to geometric variations. The lower frequency $(150 \mathrm{MHz}) \mathrm{NU}$ slow wave circuit showed some variation in phase velocity between experiment and simulation, but this variation was much smaller than for the other slow wave circuits. Small geometric variations have a greater effect on higher frequencies due to the smaller wavelength.

The CFA operation is very sensitive to the beam injection techniques. This is a rather obvious observation, but it is notable. The beam injection has a significant effect on the trajectory of the electrons throughout the CFA. There is no universal norm for proper beam injection as it depends on many of the CFA parameters such as the circuit length, current density, desired efficiency, etc. Small changes to the beam injection have a rather large effect on gain, and vigorous optimization techniques are required to maximize gain.

It was found that the entire cycloid trajectory is important to the electron beam interaction with the RF wave. Originally it was thought that the majority of the 
beam interaction with the RF wave was near the circuit and that the bottom of the cycloiding electron trajectory is not important to energy coupling. By testing the $E_{R F}=0$ distributed cathode approximation, which set the RF fields to zero within a few millimeters from the cathode/sole, the electron trajectories were significantly altered. Electrons at the bottom of the cycloid trajectory have very little kinetic energy and spend a significant amount of time at these locations. RF electric fields near the cathode/sole may be weaker than closer to the circuit, but because electrons spend much more time at the bottom of their trajectory, much of the interaction occurs near the cathode. This result not only showed that the $E_{R F}=0$ approximation is not a viable method to implement a distributed cathode, but it showed the importance of the entire electron cycloid trajectory on the CFA operation.

\subsection{Distributed Cathode Observations}

Two different types of current distributions were studied: static and time-varying. The notable results are summarized here.

\subsubsection{Static Current Distributions}

No benefit was observed from elongating the cathode emission length into the interaction region when keeping the total current constant. Longer cathodes were found to decrease the gain and efficiency of the device. Electrons emitted farther down in the interaction region couple less energy to the RF wave due to the shorter interaction length. The decreased current density when using a longer cathode was shown to

decrease the noise coupled to the RF wave; however the SNR remained constant due to the corresponding decrease in gain. The optimum electron emission profile was 
found to be one where all of the current is emitted at the beginning of the tube with proper beam optics to ensure current transmission into the interaction region with the optimum trajectory, which is simply the injected beam model.

Any possible benefits from using a static current emission profile on longer distributed cathodes would be due to the change in current density altering the beam's injection. By changing the beam emission area, the gain in some circumstances was found to increase, but only because the current density was optimized to the injection optics. One advantage with using a longer cathode is that more current can be injected into the interaction region with the large cathode area. By increasing the beam power, the gain will increase but because some electrons are injected farther down the tube, the efficiency will suffer.

\subsubsection{Time-Varying Current Distributions}

Modulating the cathode temporally at the operating frequency and spatially at the RF phase velocity pre-bunches the beam to better couple energy to the RF wave. These preformed bunches can actually drive the RF wave in the circuit. With a modulated cathode and no RF input power on the circuit, high output powers were observed with $25 \%$ efficiency. Using an RF input in combination with the modulated cathode was found to further improve operation. By injecting electrons in phase with the decelerating fields of the RF wave on the circuit, the efficiency can be further improved by $10 \%$. Tighter modulated spokes injected in the maximum decelerating field of the RF wave maximized performance but only if the current density allowed for a proper electron emission trajectory. Very high current densities have a tendency to spread out the charge bunch and promote a less efficient electron beam. 
Determination of the true gain of the device requires an estimation of the GFEA drive power. The estimation was conservatively calculated to be about $1 \mathrm{~W}$ based on the GFEA devices from [25-27]. Because the NU CFA is a low power device, this drive power is about $1 \%$ of the total possible output power, which prevents an direct prediction of small-signal gain. In order to estimate small-signal gains, the estimated power was reduced by one order of magnitude to $0.1 \mathrm{~W}$ in order to mimic a higher power device. Because of the exponential relationship between gate-to-emitter voltage and current of GFEAs, a small increase in RF drive power creates a much larger increase in beam power. In higher power devices, the GFEA drive power could be much less than the output power.

\subsection{Method Comparison}

Four methods for electron beam injection were tested: static injected beam, modulated injected beam, static distributed beam, and a modulated distributed beam. All the methods behave differently at different beam and RF powers, so comparison

of the methods are divided into two main parts: small-signal and high power. The small-signal regime is when the RF input power is $<1 \%$ of the maximum obtainable output power, and the high power regime is when the RF input power is $>1 \%$ of the maximum obtainable output power.

In the small-signal regime, the time-varying distributed method greatly outperformed both static methods with the time-varying injected beam variation close behind. The low power NU CFA design slightly clouds this observation, but by using the low GFEA drive power estimate, the result is clear. The modulated cathodes has $\approx 12 \mathrm{~dB}$ greater gain, is $\approx 20 \%$ more efficient, and has a $20 \mathrm{~dB}$ greater SNR than 
the unmodulated case. Generally, as discovered from the distributed cathode study, emitting more electrons closer to the beginning of the tube, as in the injected beam configuration, is more efficient than a distributed beam, but the high current densities required for the modulated injected beam prevented optimum injection into the tube. By using better beam optics, it is expected that the modulated injected beam will outperform the distributed beam variant when using the same currents.

In the high power regime, the modulated cathodes still outperform the unmodulated cathodes, but the performance of all the methods start to converge. The gain converges to $\approx 8 \mathrm{~dB}$ and the efficiency to $\approx 30 \%$. As the RF drive power on the circuit increases, the efficiency increases for all the devices. The higher RF field in the interaction region has a much greater impact on the electrons in the interaction region, and can extract more energy from them. This diminishes the effectiveness of pre-bunching the electron beam at higher RF powers. The main advantage of pre-bunching the beam is in the SNR. The SNR of the modulated cathode device is $15 \mathrm{~dB}$ greater than the unmodulated one. This is an inherent advantage in itself but this low noise may allow for higher gains and higher output powers in higher power devices. The gain and output power of the device is partly limited by noise in the device exciting unwanted modes in the device. Lowering the noise will help in this regard.

The modulated injected beam performed rather well compared with the distributed modulated beam. However, the distributed beam has one major advantage over the injected beam: the ability to inject more current. The maximum beam current in the injected beam configuration is much lower than the distributed beam configuration due to the higher current density. This limits the use of the injected beam to relatively lower power devices. 


\subsection{Modulated Distributed Cathode Importance}

The modulated distributed cathode has the capability to increase the maximum output power while increasing the gain at these high powers. Current high power CFAs $(>1 \mathrm{MW})$ have very low gain $(<15 \mathrm{~dB})$ due to a fundamental mode-locking limit. In order to retain lock on the main amplifying mode, the RF drive power must be increased. Using a modulated cathode induces its own mode locking mechanism, which will improve the gain at these high powers. Also, the modulated cathode helps to reduce the noise in the device, further expanding its usefulness to low noise applications and may help to increase the maximum achievable power.

Implementing a modulated distributed cathode in an actual high power device is fraught with many difficulties, but there are ways to achieve this goal. A few different methods are described in Sec. 7.8.2. One important point here is that not all the beam current needs to be supplied by the GFEA. Conventional cathodes could be used to produce the bulk of the current, and the modulated GFEAs could be used to supplement the current and provide mode-locking. A direct approach using the distributed cathode, where all the current is supplied by the GFEAs, could also be used, but the implementation is much more difficult at higher powers. An injected beam approach can immediately benefit from using modulated GFEAs and is a relatively simple implementation. This is an important contribution, but it may not replace well established MVEDs in this lower power regime.

\subsection{Future Work}

The study in this dissertation showed some significant improvements to the smallsignal-gain when using the modulated cathode. The gain observed in the higher power 
regime was improved only marginally in this low power device. The goal of future work would be to study the effect of a modulated cathode on mode interference at higher powers. There are two ways to achieve this goal. One way would be to continue with the linear format NU CFA used in this work, and the other way would be to shift to a well established cylindrical high power design. The more desirable option would be to use a well established cylindrical design to alleviate the developmental overhead that will be required when altering the NU CFA design to higher power.

Whichever model that will be used, the study will focus first on the characterization of the maximum achievable output power of the device and the mode-locking mechanism via simulation. The next step would be to study the effect of modulated current on the mode-locking mechanism. This study will contribute fundamental knowledge on the physics of the device and any operation improvements will motivate further study in the field.

The next phase of research will be on the implementation in high power devices. This is where the end hat injection, and distributed GFEA assisted secondary emitting methods can be simulated. These two methods seem to be the least complex methods to implement a modulated cathode experimentally. A non-reentrant CFA design could also be simulated to test the injected GFEA assisted secondary emitting methods. Direct approaches would require more research in hop funnels or lateral emitters. These approaches would have a much higher payout if they work, but would require much more research. 


\section{APPENDIX A}

\section{CFA EXPERIMENTAL SETUP}

\section{A.1 Measurement and Control Setup}

Figure A.1 shows the measurement and control setup. The potential difference between the anode and the sole is on the order of a $1000 \mathrm{~V}$. The choice was made to have the anode at the earth ground potential and the cathode to be at the negative potential. 


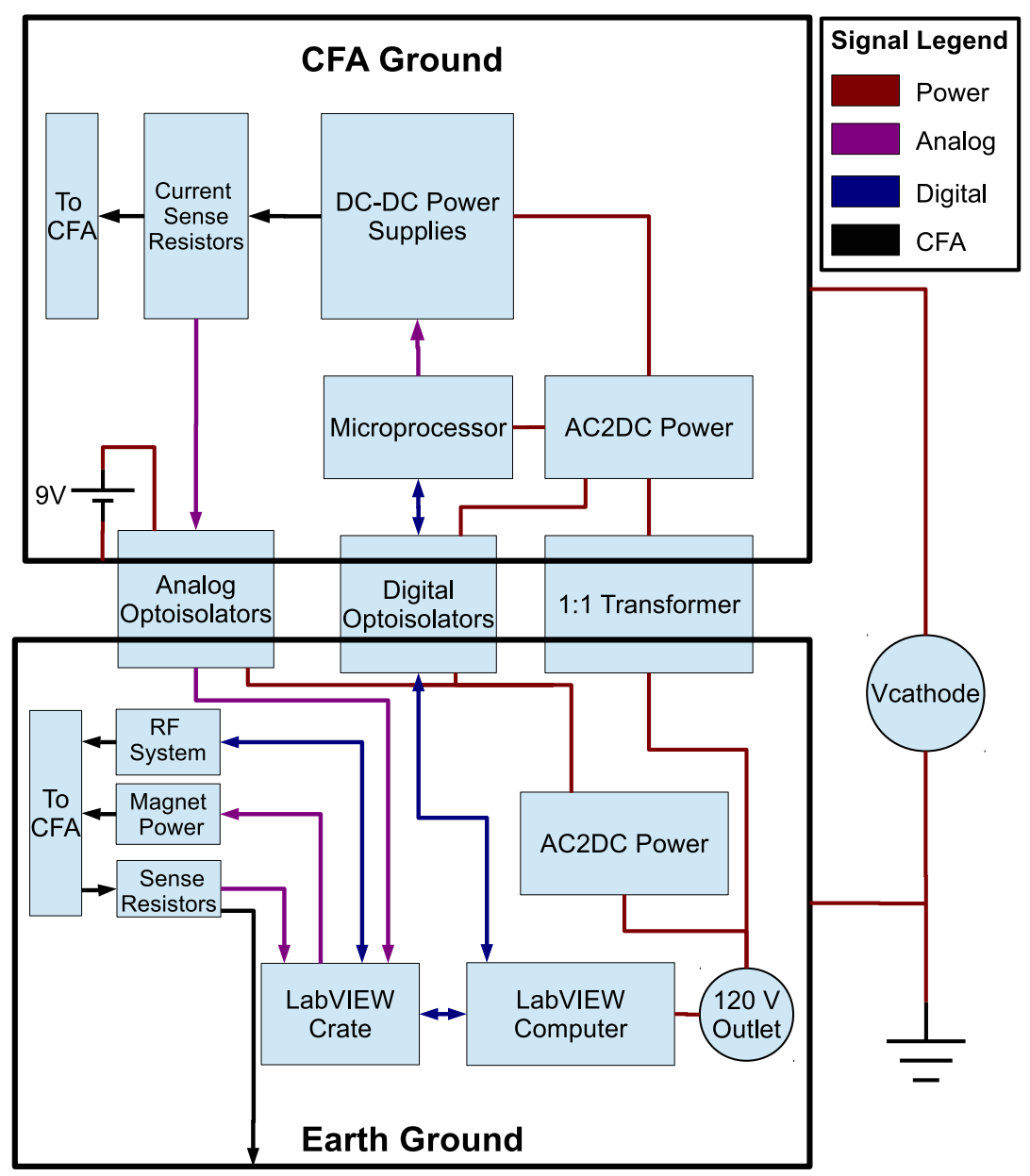

Figure A.1: Measurement setup schematic. There are two potentials at which signals are measured, earth ground and CFA ground. Recording earth ground measurements is easily done by LabVIEW data acquisition (DAQ) Crate. CFA ground based measurements transmit the measurement signal through analog opto-isolaters. Control of CFA ground based currents and voltages is done by a CFA ground based microcontroller which communicates to the earth ground computer through digital optoisolators.

There are two distinct grounds in the diagram: earth ground and CFA ground. Earth ground is the potential for the computer, the RF circuitry, the analog to digital converters, and the magnets. CFA ground is the potential for the CFA and all its 
measurement and control. Communication between the two potentials is performed through opto-isolators. The CFA cathode operates at the CFA potential and requires extensive circuitry to control power sources, DC-DC converters, circuitry to control the DC-DC converters, and the opto-isolators to communicate signals.

The RF system diagram is shown in Fig. A.2. The input and output power of the CFA is measured via a $-20 \mathrm{~dB}$ signal from the RF directional coupler into a spectrum analyzer.

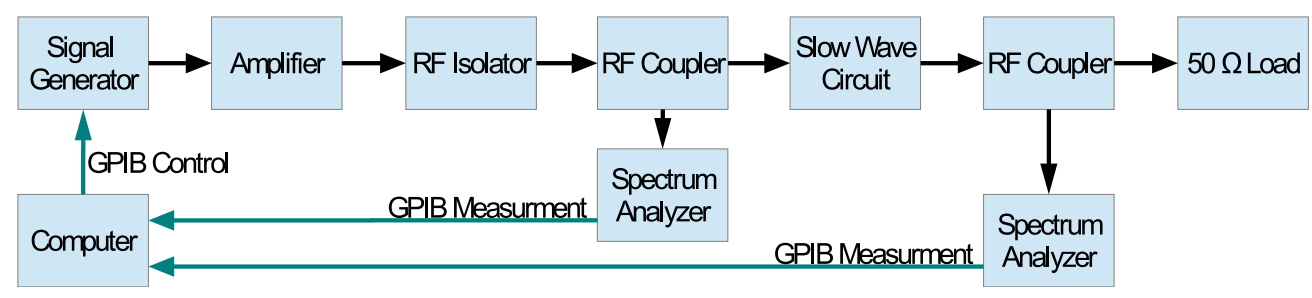

Figure A.2: RF system flow

\section{A.1.1 CFA Ground}

The sole and cathode must be $\approx-3000 \mathrm{~V}$ relative to the slow wave circuit. CFA ground is biased using a $5000 \mathrm{~V}, 300 \mathrm{~mA}$ Glassman power supply. At this potential are the cathode, hop, sole, and end hats. The current control and monitoring of these electrodes must be performed at this potential. Power to the electrodes is performed with DC-DC supplies, which have an analog control voltage. The control voltage is controlled by a microcontroller board. The microcontroller communicates via a serial connection through opto-isolators to the LabVIEW computer.

Each electrode is powered by the DC-DC converters through current sense resistors. Current monitoring is performed by current sense resistors. To measure the voltage across the resistor, opto-isolator boards used to measure the voltage and pass 
the voltage to the LabVIEW crate. The LabVIEW crate converts the analog voltage to a digital value and passes the data to the LabVIEW computer.

Ideally, to measure the emitted current the difference between the gate current and the emitter current would be considered emitted current. Since the leakage current between the gates and emitters is 2-3 orders of magnitude higher than the emitted current, the voltage created by the current is on the noise level.

All the power for the DC-DC converters and the microcontroller board is given by a 1:1 AC transformer. The $120 V_{r m s}$ power from the wall outlet is isolated through the transformer, and then AC-DC converters are used to get the $12 \mathrm{~V}$ power needed by the DC-DC converters and the microcontroller board.

The FEA is very susceptible to damage from arcs and the potential for arcs is very high from the $\approx 3000 \mathrm{~V}$ potentials and the beam current. To monitor for arcs, high voltage capacitors are used at various places on the CFA ground. At a minimum, the gate of the FEA is monitored. One side of the caps are on a node of the CFA ground potential, and the other side is connected to an oscilloscope at the earth ground potential. Any displacement current created by an arc can be detected in this manner. Sometimes the arcs occur multiple times at a high frequency, and the system can be shut down to prevent further potential damage to the FEA or equipment.

\section{Microcontroller Board}

The microcontroller inside the CFA potential allows for an extensive system of measurement and control. The tasks of the microcontroller board are to control the DC-DC supplies and to monitor and control the emitter currents. Currently the microprocessor board is only used to control the voltages on the DC-DC supply and all measurements use the DAQ. Currently, the MP board can only control 4 
DC-DC sources. In the future with distributed cathode, the microprocessor board in conjunction with a current control board will control the voltages to different sections to the cathode.

The microprocessor board communicates to the LabVIEW computer via 2 serial communications, one command channel and one measurement channel. The serial communication goes through digital opto-isolators and then to a serial to USB chip (FTDI) and to the computer. The command channel takes commands from the computer in order to change the voltages to the DC-DC. The microcontroller constantly broadcasts any measurements on measurement channel. The LabVIEW computer just reads the measurements and updates the LabVIEW display when it can.

\section{DC-DC Converters}

The DC-DC converters used in this experiment are UltraVolt. The supply can be a either a negative or a positive supply. The maximum current is on the order of $50 \mathrm{~mA}$ and the maximum voltage magnitude is 500,1000 , or $2000 \mathrm{~V}$ depending on the model. They require a $12 \mathrm{~V}$ power source and $0-5 \mathrm{~V}$ analog signal is used to control the output voltage. For the negative supplies, $5 \mathrm{~V}$ on the input signal corresponds to $0 \mathrm{~V}$ on the output, and $0 \mathrm{~V}$ corresponds to the maximum negative voltage. For the positive supplies, $0 \mathrm{~V}$ corresponds to a $0 \mathrm{~V}$ output and $5 \mathrm{~V}$ corresponds to the maximum.

These supplies also have some non-idealities. One non-ideality is the fact that depending on the polarity of the supply, current can only go one way for it to work correctly. For example, the positive supply operates correctly if it sources current rather than sinking current. If for some reason current tries to go into the source, the voltage will float up due to the inability to properly sink the current. The negative 
supply can only sink current rather than source current. This is a problem because for example, if the electrode is at a negative potential and electrons in the CFA manage to collect on the electrode for whatever reason, the source would have difficulty sinking these electrons and the electrode would float negatively. This problem can alter the results due to fluctuations in electrode voltages, and care must be given in order for this problem not to occur. Usually, the electrodes are biased negatively enough in order to make it impossible for electrons to collect on them.

\section{A.1.2 Earth Ground}

The earth ground side is the potential at which the slow wave circuit, the end collector, the RF circuitry, and the LabVIEW computer are. The electrode currents are monitored at this potential, and the RF control, and the data management is done at this potential.

To measure the current on the anode and the end collector, the voltage across current sense resistors is monitored and then digitally converted through the DAQ crate directly. The voltage across the resistor is already relative to ground, therefore no opto-isolators are needed.

\section{RF System}

Fig. A.3 shows the the RF connections. The signal generator generates the RF wave at the operating frequency $(400-1000 \mathrm{MHz})$. The amplifier amplifies the signal to the appropriate amplitude $(1-10 \mathrm{~W})$ in order to facilitate a proper interaction with the electron beam. The RF power should be less than the electron beam power with a ratio of 1:2-1:10 RF power to beam power. This $R F$ signal goes through an $R F$ isolator to prevent any unwanted high power reflections from damaging the amplifier. 
The signal then goes to an RF directional coupler which outputs a $-20 \mathrm{~dB}$ version of the signal and allows for a safe measurement of the input power of the CFA through a spectrum analyzer. Then the signal goes to the slow wave circuit which is designed to be $50 \Omega$ and requires no matching circuit. The signal then goes to an RF directional coupler to measure the output power of the CFA through a spectrum analyzer. This power measurement is sent to the computer via GPIB and recorded. The main RF signal is then dissipated in a $50 \Omega$ load.

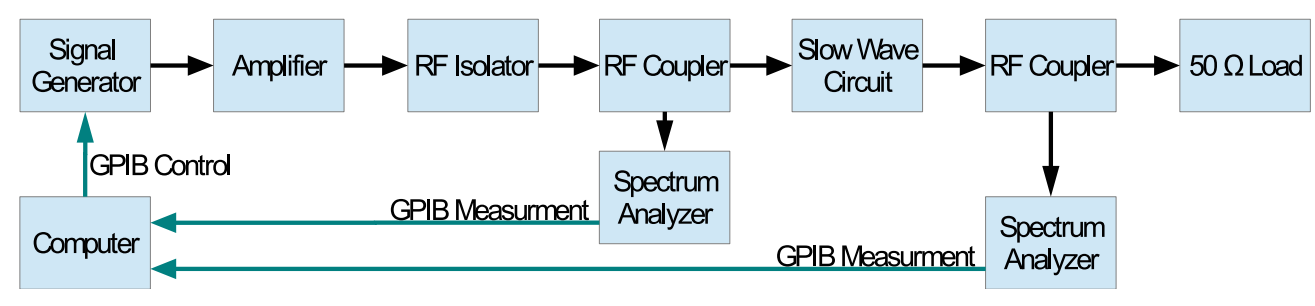

Figure A.3: RF system flow

In order to ensure that the slow wave circuit is $50 \Omega$, a network analyzer is used. The input and output of the slow wave circuit is connected to the network analyzer to determine the impedance. The impedance usually fluctuates widely across the 400-1000 MHz frequency range, but a few distinct frequencies cross right at $50 \Omega$, and these are the chosen operating frequencies.

\section{LabVIEW Crate}

The experimental system needs to measure and record all the currents, to communicate with various equipment via GPIB and to control the magnet power supply via a analog voltage. The LabVIEW crate is used to complete these tasks.

The LabVIEW DAQ system connects to the computer via a PCI connection. The crate contains a GPIB connector card and 2 measurement cards. These measurement 
cards have many functions, but the functions used in this work are the analog inputs

and outputs. Each measurement card has a 1 ADC converter which can monitor 16 channels and 2 DACs. This allows a maximum of 32 analog voltage measurements and 4 analog outputs.

In order for $1 \mathrm{~A} 2 \mathrm{D}$ converter to monitor 16 channels it uses a multiplexer. While being able to monitor 16 analog signals is convenient, the multiplexing nature requires precautions to minimize error. The voltage on the channel read right before has a tendency to couple to the next channel read, and can alter the reading. Subsequent voltage measurements that are significantly different $(>1 \mathrm{~V})$ can alter the reading. To prevent this, the order of the subsequent reads are ensured that the magnitude of the voltages are close.

\section{A.1.3 Opto-Isolators}

Opto-isolators utilize LEDs and photodiodes to transmit small signals across potentials. The opto-isolators used in this work are the LOC110 chips. These chips have one LED and two photodiodes internally. In conjunction with 2 OP-Amps, the circuit can transfer voltage signals across potential differences up to 5000 V. Fig. A.4 shows the general schematic used in this work and demonstrates how they function 


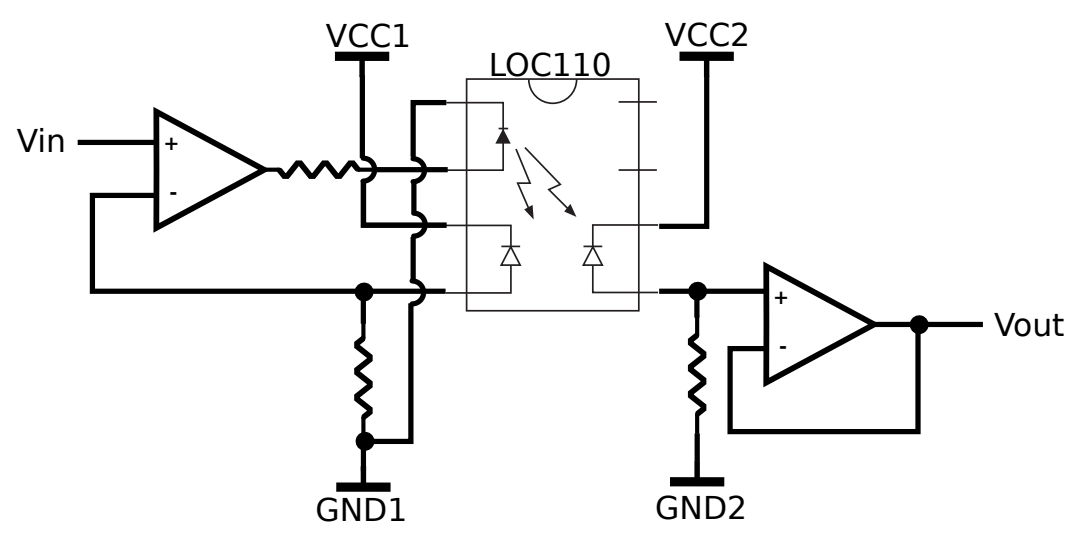

Figure A.4: Opto-isolator schematic

Photodiodes generate a current when light hits them. To create photodiodes on different wafers, and on different locations on the wafer with identical characteristics is difficult, therefore a feedback mechanism is needed to ensure proper signal transfer. In Fig. A.4, the LOC110 chip has 2 photodiodes, one for feedback and one for the output. The 2 photodiodes on this chip have identical characteristics due to being processed near each other and on the same wafer. The input signal goes into the op-amp, which generates a current through the LED. Light from the LED is transmitted to both photodiodes. Current generated in the feedback photodiode goes through the resistor, generating a feedback voltage. The op-amp adjusts the current though the LED in order to match the feedback voltage to the input voltage. Since the feedback photodiode and the output photodiode are identical, the proper output voltage is observed on $V_{\text {out }}$.

Using this general schematic, PCB boards were designed to transfer signal voltages across potentials. When measuring currents, the output voltage goes to the DAQ crate which is at earth ground. Vcc2 was generated by a $12 \mathrm{~V}$ supply. The measurement side is at CFA ground plus the electrode voltage. Vcc1 is generated by a $9 \mathrm{~V}$ battery in these cases. Due to a low power requirement of all components on the board, the 
$9 \mathrm{~V}$ battery lasts $>24$ hours of use. These opto-isolator boards can measure signals up to the power supply voltage, in this case $9 \mathrm{~V}$.

\section{A.2 Experimental Procedure}

To test the CFA configuration, the RF wave and electron beam are turned on, and the output $\mathrm{RF}$ wave is observed for amplification. The $\mathbf{E} \times \mathbf{B}$ velocity of the electron beam is swept to find the synchronous condition where amplification should occur. The following procedure is used:

1. Connect the SW circuit to ground to act as an anode

2. Burn in the cathode to get maximum current at the lowest gate voltage. This also removes contaminates from the interaction region. It is safer to remove the contaminates at a controlled rate and at the lowest energy possible

3. Characterize the beam trajectory. Look for the best operating condition where the most of the beam is going down the tube to the end collector and with a $\mathrm{E}$ cross $\mathrm{B}$ velocity close to the predicted RF phase velocity.

4. Setup the RF system: choose optimum frequency where the SW circuit is matched using the network analyzer

5. Choose a Beam power that is 3-10 times greater than the RF wave.

6. Turn on the RF wave

7. Observe the output power of the RF wave on the spectrum analyzer

8. Turn on the electron beam 
9. Observe the spectrum analyzer for an increase in output power

10. Alter the potential of the CFA ground, which alters the electric field in the interaction region. This changes the $\mathbf{E} \times \mathbf{B}$ velocity of the beam. This is how the beam velocity is swept to find the synchronous condition. If the electron beam deviates from the end collector due to the change in the electric field, the magnetic field can be adjusted to 'realign' the beam. This changes the average beam velocity, but an effective sweep of the beam velocity can be done while maintaining the same end collector current can be done in this manner. 


\section{Bibliography}

[1] Samuel Y. Liao. Microwave Electron-Tube Devices. Prentice Hall, 1988.

[2] A. S. Gilmour Jr. Klystrons, Traveling Wave Tubes, Magnetrons, Crossed-Field Amplifiers, and Gyrotrons. Artech House, 2011.

[3] A. S. Gilmour Jr. Microwave Tubes. Artech House, 1986.

[4] Robert J. Barker, Neville C. Luhmann, John H. Booske, and Gregory S. Nusinovich. Modern Microwave and Millimeter-Wave Power Electronics. Wiley, 2005.

[5] C. E. Holland, A. Rosengreen, and C. A. Spindt. "A study of field emission microtriodes". In: Electron Devices Meeting, 1990. IEDM '90. Technical Digest., International. Dec. 1990, pp. 979-982. DOI: 10.1109/IEDM.1990.237076.

[6] S.G. Bandy, M. C. Green, C.A. Spindt, M.A. Hollis, W.D. Palmer, et al. "Application of gated field emitter arrays in microwave amplifier tubes". In: Vacuum Microelectronics Conference, 1998. Eleventh International. July 1998, pp. 132-133. DOI: 10.1109/IVMC.1998.728677.

[7] A. S. Gilmour Jr. Principles of Traveling Wave Tubes. Artech House, 1994.

[8] D.R. Whaley, B.M. Gannon, C.R. Smith, C.M. Armstrong, and C.A. Spindt. "Application of field emitter arrays to microwave power amplifiers". In: Plasma Science, IEEE Transactions on 28.3 (June 2000), pp. 727-747. ISSN: 0093-3813. DOI: $10.1109 / 27.887712$. 
[9] D.R. Whaley, R. Duggal, C.M. Armstrong, C.L. Bellew, C.E. Holland, et al. "100 W Operation of a Cold Cathode TWT". In: Electron Devices, IEEE Transactions on 56.5 (May 2009), pp. 896-905. ISSN: 0018-9383. DOI: 10 . 1109/TED . 2009. 2015614.

[10] J. R. Pierce. "Traveling-Wave Tubes". In: Bell System Technical Journal 29.3 (1950), pp. 390-460. ISSN: 1538-7305. DOI: 10 . 1002/ j . 1538-7305 . 1950 . tb02352 . x. URL: http : / / dx . doi . org/10 . 1002 / j . 1538-7305 . 1950 . tb02352.x.

[11] Mikhail Fuks and Edl Schamiloglu. "Rapid Start of Oscillations in a Magnetron with a "Transparent" Cathode". In: Phys. Rev. Lett. 95 (20 Nov. 2005), p. 205101. DOI: 10.1103/PhysRevLett.95.205101. URL: http://link.aps . org/doi/10.1103/PhysRevLett.95.205101.

[12] H.L. Bosman, M.I. Fuks, S. Prasad, and E. Schamiloglu. "Improvement of the output characteristics of magnetrons using the transparent cathode". In: Plasma Science, IEEE Transactions on 34.3 (June 2006), pp. 606-619. ISSN: 0093-3813. DOI: 10.1109/TPS.2006.875771.

[13] Sulmer Fernandez Gutierrez. "Simulation of a Magnetron Using Discrete Modulated Current Sources". PhD thesis. Boise State University, May 2014.

[14] J. Browning, Chung Chan, J.Z. Ye, and Thomas E. Ruden. "A low-frequency crossed-field amplifier for experimental investigations of electron-radio frequency wave interactions". In: Plasma Science, IEEE Transactions on 19.4 (Aug. 1991), pp. 598-606. ISSN: 0093-3813. DOI: 10.1109/27.90324.

[15] J. Browning, C. Chan, J. Z. Ye, G. E. Dombrowski, and T. E. Ruden. "Electron plasma and wave measurements in a crossed-field amplifier and comparison 
with numerical simulation". In: IEEE Transactions on Electron Devices 39.10 (Oct. 1992), pp. 2401-2407. ISSN: 0018-9383. DOI: 10.1109/16.158815.

[16] R. G. Lock. "A non-reentrant crossed-field amplifier with cycloiding injected beam". In: Electron Devices Meeting, 1968 International. Vol. 14. 1968, pp. 2626. DOI: $10.1109 /$ IEDM. 1968.187952.

[17] John F. Skowron. "The Continuous-Cathode (Emitting-Sole) Crossed-Field Amplifier". In: PROCEEDINGS OF THE IEEE 61.3 (Mar. 1973), pp. 330-356.

[18] D. V. Sokolov and D. I. Trubetskov. "Microelectronic field-emission crossedfield amplifier with two delay lines". In: Vacuum Microelectronics Conference, 1996. IVMC'96., 9th International. July 1996, pp. 601-605. DOI: 10.1109 / IVMC . 1996.601895.

[19] M. L. Cooke, E. K. Shaw, and L. I. Yarrington. "The crossed-field amplifier computational and experimental results". In: Electron Devices Meeting, 1973 International. Vol. 19. 1973, pp. 278-281. DOI: 10.1109/IEDM.1973.188707.

[20] M. Garven, S.J. Cooke, A.W. Cross, A.D.R. Phelps, and S.N. Spark. "Gyrotron experiments employing a field emission array cathode". In: Plasma Science, 1995. IEEE Conference Record - Abstracts., 1995 IEEE International Conference on. June 1995, pp. 136-. DOI: 10.1109/PLASMA.1995.531563.

[21] Z. Duan, F. Wang, X. Tang, Y. Wang, Y. Gong, et al. "Overview of vacuum electron devices for biomedical applications". In: 2015 IEEE MTT-S 2015 International Microwave Workshop Series on RF and Wireless Technologies for Biomedical and Healthcare Applications (IMWS-BIO). Sept. 2015, pp. 156157. DOI: $10.1109 /$ IMWS-BIO. 2015.7303825. 
[22] S. M. Hanna. "Role of Microwave Accelerators in Cancer Treatment". In: 2007 IEEE/MTT-S International Microwave Symposium. June 2007, pp. 1337-1340. DOI: $10.1109 /$ MWSYM. 2007.380459.

[23] C.A. Spindt, I. Brodie, L. Humphrey, and E.R. Westerberg. "Physical properties of thin-film field emission cathodes with molybdenum cones". In: Journal of Applied Physics 47.12 (Dec. 1976), pp. 5248-5263. ISSN: 0021-8979. DOI: $10.1063 / 1.322600$.

[24] Dorota Temple. "Recent progress in field emitter array development for high performance applications". In: Materials Science and Engineering: R: Reports 24.5 (1999), pp. 185-239. ISSN: 0927-796X. DOI: 10 .1016/S0927-796X (98) 00014-X. URL: http://www.sciencedirect.com/science/article/pii/ S0927796X9800014X.

[25] S. A. Guerrera and A. I. Akinwande. "Silicon Field Emitter Arrays With Current Densities Exceeding $100 \mathrm{~A} / \mathrm{cm} 2$ at Gate Voltages Below 75 V'. In: IEEE Electron Device Letters 37.1 (Jan. 2016), pp. 96-99. ISSN: 0741-3106. DOI: $10.1109 /$ LED . 2015.2499440.

[26] S A Guerrera and A I Akinwande. "Nanofabrication of arrays of silicon field emitters with vertical silicon nanowire current limiters and self-aligned gates". In: Nanotechnology 27.29 (2016), p. 295302. URL: http://stacks.iop.org/ $0957-4484 / 27 / i=29 / a=295302$.

[27] S. A. Guerrera and A. I. Akinwande. "High performance and reliable silicon field emission arrays enabled by silicon nanowire current limiters". In: 2015 IEEE International Electron Devices Meeting (IEDM). Dec. 2015, pp. 33.1.133.1.4. DOI: 10.1109/IEDM. 2015.7409818. 
[28] J. Benford. "History and future of the relativistic magnetron". In: 2010 International Conference on the Origins and Evolution of the Cavity Magnetron. Apr. 2010, pp. 40-45. DOI: 10.1109/CAVMAG. 2010.5565566.

[29] B. A. Kerr, D. Shiffler, T. Knight, M. LaCour, K. Golby, et al. "Carbon velvet cathode implementation on the Orion relativistic magnetron". In: 2005 The IEE Pulsed Power symposium (Reg. No. 2005/11070). Sept. 2005, pp. 6/1-6/6. DOI: $10.1049 /$ ic : 20050035 .

[30] G.N Fursey. "Field emission in vacuum micro-electronics". In: Applied Surface Science 215.1-4 (2003). \{IVESC\} 2002, pp. 113-134. ISSN: 0169-4332. DOI: http : / / dx . doi . org / 10 . 1016 / S0169 - 4332(03 ) 00315 - 5. URL: http : //www.sciencedirect.com/science/article/pii/S0169433203003155.

[31] Robert K. Parker. EXPLOSIVE ELECTRON EMISSION AND THE CHARACTERISTICS OF HIGH-CURRENT ELECTRON FLOW. Tech. rep. Air Force Weapons Laboratory, Kirtland Air Force Base, New Mexico, Feb. 1974. URL: http://www.dtic.mil/dtic/tr/fulltext/u2/775992.pdf.

[32] C.A. Spindt, C.E. Holland, P.R. Schwoebel, and I. Brodie. "Field-emitter-array development for microwave applications". In: Electron Devices Meeting, 1995. IEDM '95., International. Dec. 1995, pp. 389-392. DOI: 10.1109/IEDM. 1995. 499221.

[33] I. Brodie and P.R. Schwoebel. "Vacuum microelectronic devices [and prolog]". In: Proceedings of the IEEE 82.7 (July 1994), pp. 1006-1034. ISSN: 0018-9219. DOI: $10.1109 / 5.293159$.

[34] B.H.W. Hendriks, G.G.P van Gorkom, N. Lambert, and S.T. de Zwart. "Modes in electron-hopping transport over insulators sustained by secondary electron 
emission". In: J. Phys. D: Appl. Phys. 30.8 (Apr. 1997), pp. 1252-1264. DOI: $10.1088 / 0022-3727 / 30 / 8 / 016$.

[35] B. H. W. Hendriks, G. G. P. van Gorkom, A. T. M. H. van Keersop, N. Lambert, and S. T. De Zwart. "Dynamical behavior of electron hop transport over insulating surfaces". In: Journal of Applied Physics 85.3 (Feb. 1999), pp. 1848-1856. ISSN: 0021-8979. DOI: 10.1063/1.369309.

[36] C. Lester, J. Browning, and L. Matthews. "Electron-Hop-Funnel Measurements and Comparison With the Lorentz-2E Simulation". In: Plasma Science, IEEE Transactions on 39.1 (Jan. 2011), pp. 555-561. ISSN: 0093-3813. DOI: 10.1109/ TPS . 2010.2087775.

[37] Marcus Pearlman. "Investigation of the Current Transmission Hysteresis in Electron Hop Funnels". MA thesis. Boise, ID USA: Dept. of Electrical and Computer Engineering, Boise State Univ., 2012.

[38] Tyler Rowe, Marcus Pearlman, and Jim Browning. "Hysteresis in experimental I-V curves of electron hop funnels". In: Journal of Vacuum Science \& Technology B, Nanotechnology and Microelectronics: Materials, Processing, Measurement, and Phenomena 31.4 (2013), p. 042204. DOI: 10 . $1116 / 1$. 4813779. eprint: http : / /dx. doi .org/10 . 1116/1 . 4813779. URL: http: //dx.doi.org/10.1116/1.4813779.

[39] Jim Browning, Charles Lester, and John Luginsland. "Development of a "Smart" Crossed-field Amplifier". In: IEEE 36th International Conference of Plasma Science. (San Diego, USA). June 2009, p. 347. ISBN: 978-1-4244-2617-1.

[40] SIMION. http://simion.com/.

[41] COMSOL. http://www.comsol.com/. 
[42] Tech-X. http://www.txcorp.com/.

[43] A. H. W. Beck. Space-Charge Waves. Pergamon Press, 1958.

[44] Ciersiang Chua and S. Aditya. "A 3-D U-Shaped Meander-Line Slow-Wave Structure for Traveling-Wave-Tube Applications". In: Electron Devices, IEEE Transactions on 60.3 (Mar. 2013), pp. 1251-1256. ISSN: 0018-9383. DOI: 10. 1109/TED . 2013. 2241438.

[45] J. Arnaud. "Periodic Structures". In: Crossed-Field Microwave Devices. Ed. by E. Okress, G. Mourier, J. Feinstein, and E. Kettlewell. Vol. 1. London: Academic Press Inc., 1961.

[46] G. Döhler, D. Gagne, D. Gallagher, and R. Moats. "Serpentine waveguide TWT"'. In: Electron Devices Meeting, 1987 International. Vol. 33. 1987, pp. 485488. DOI: $10.1109 /$ IEDM. 1987.191465.

[47] M. J. Clark. "Slow Wave Circuits for Miniature Crossed Field Amplifiers". In: Microwave Conference, 1981. 11th European. Sept. 1981, pp. 645-649. DOI: 10.1109/EUMA. 1981.332920.

[48] F. Shen, Y. Wei, X. Xu, Y. Liu, M. Huang, et al. "U-shaped microstrip meander-line slow-wave structure for Ka-band traveling-wave tube". In: Microwave and Millimeter Wave Technology (ICMMT), 2012 International Conference on. Vol. 1. May 2012, pp. 1-2. DOI: 10.1109/ICMMT .2012.6229942.

[49] F. Shen, Y. Wei, H. Yin, Y. Gong, X. Xu, et al. "A Novel V-Shaped Microstrip Meander-Line Slow-Wave Structure for W-band MMPM". In: IEEE Transactions on Plasma Science 40.2 (Feb. 2012), pp. 463-469. ISSN: 0093-3813. DOI: 10.1109/TPS. 2011.2175252. 
[50] S. Sengele, H. Jiang, J. H. Booske, C. L. Kory, D. W. van der Weide, et al. "Microfabrication and Characterization of a Selectively Metallized W-Band Meander-Line TWT Circuit". In: IEEE Transactions on Electron Devices 56.5 (May 2009), pp. 730-737. ISSN: 0018-9383. DOI: 10.1109/TED . 2009. 2015416.

[51] Albert W. Hull. "The Effect of A Uniform Magnetic Field on the Motion of Electrons Between Coaxial Cylinders." In: Phys. Rev. 18 (1 July 1921), pp. 31-57. DOI: 10.1103/PhysRev.18.31. URL: http://link.aps.org/doi/ 10.1103/PhysRev.18.31.

[52] O. Buneman and G. Mourier. "Dynamic Phenomena: Interaction of Beams and Circuits". In: Crossed-Field Microwave Devices. Ed. by E. Okress, G. Mourier, J. Feinstein, and E. Kettlewell. Vol. 1. London: Academic Press Inc., 1961, pp. $367-438$.

[53] R. J. Espinosa and R. R. Moats. "Broad-band injected-beam crossed-field amplifiers". In: IEEE Transactions on Electron Devices 24.1 (Jan. 1977), pp. 1321. ISSN: 0018-9383. DOI: $10.1109 / \mathrm{T}-\mathrm{ED} .1977 .18671$.

[54] E. K. Shaw. "Starting in the cold cathode distributed emission crossed field amplifier". In: IEEE Transactions on Electron Devices 24.1 (Jan. 1977), pp. 2226. ISSN: 0018-9383. DOI: 10.1109/T-ED.1977.18672.

[55] A. Shih, J. Yater, C. Hor, and R. H. Abrams. "Secondary electron emission properties of oxidized beryllium CFA cathodes". In: IEEE Transactions on Electron Devices 41.12 (Dec. 1994), pp. 2448-2454. ISSN: 0018-9383. DOI: 10. 1109/16. 337462 .

[56] M. S. Worthington, C. L. Wheeland, K. Ramacher, and E. Doyle. "Composite and diamond cold cathode materials". In: IEEE Conference Record - Abstracts. 
1996 IEEE International Conference on Plasma Science. June 1996, pp. 235-. DOI: $10.1109 /$ PLASMA. 1996.551449.

[57] Carlos Alberto Fonzar Pintão and Roberto Hessel. "Total secondary-electron yield of metals measured by a dynamic method". In: Journal of Applied Physics 88.1 (2000), pp. 478-482. DOI: 10.1063/1.373682. eprint: http://dx.doi . org/10.1063/1.373682. URL: http://dx.doi.org/10.1063/1.373682.

[58] A. Dallos, J. F. Skowron, and R. Dang. "Effects of ionized oxygen on primary and secondary emission, and total current of a CFA". In: IEEE Transactions on Electron Devices 34.5 (May 1987), pp. 1201-1208. ISSN: 0018-9383. DOI: 10.1109/T-ED . 1987.23067.

[59] L3 Communications Electron Deviced Division. http: //www2 . 1-3com.com/ edd/.

[60] Integrated Engineering Software. http://www. integratedsoft.com/.

[61] H. A. van der Vorst. "BI-CGSTAB: A Fast and Smoothly Converging Variant of BI-CG for the Solution of Nonsymmetric Linear Systems". In: SIAM J. Sci. Stat. Comput. 13.2 (Mar. 1992), pp. 631-644. ISSN: 0196-5204. DOI: 10.1137/ 0913035. URL: http://dx.doi.org/10.1137/0913035.

[62] P.R. Amestoy, I.S. Duff, and J.-Y. L'Excellent. "Multifrontal parallel distributed symmetric and unsymmetric solvers". In: Computer Methods in Applied Mechanics and Engineering 184.2-4 (2000), pp. 501-520. ISSN: 0045-7825. DOI: http: //dx .doi .org/10.1016/S0045-7825(99)00242-X. URL: http: //www.sciencedirect.com/science/article/pii/S004578259900242X.

[63] Jr. David M. Young. "Iterative Methods for Solving Partial Difference Equations of Elliptic Type". PhD thesis. Havard University, May 1950. 
[64] David J. Manura and David A. Dahl. SIMION Version 8.0 User Manual. English. Version Version 8.0.4. Scientific Instrument Services, Inc.

[65] C. K. Birdsall and A. B. Langdon. Plasma Physics VIA Computer Simulation. Taylor \& Francis, 2005.

[66] Nicholas Metropolis and S. Ulam. "The Monte Carlo Method". In: Journal of the American Statistical Association 44.247 (1949), pp. 335-341. ISSN: 01621459. URL: http: //www . jstor . org/stable/2280232.

[67] VSim Reference Manual. English. Version Version 7.2.0. TechX. 321 pp.

[68] H. A. van der Vorst. "Bi-CGSTAB: A Fast and Smoothly Converging Variant of Bi-CG for the Solution of Nonsymmetric Linear Systems". In: SIAM Journal on Scientific and Statistical Computing 13.2 (1992), pp. 631-644. DOI: 10 . 1137/0913035. eprint: http://dx.doi .org/10.1137/0913035. URL: http: //dx.doi.org/10.1137/0913035.

[69] Youcef Saad and Martin H. Schultz. "GMRES: A Generalized Minimal Residual Algorithm for Solving Nonsymmetric Linear Systems". In: SIAM Journal on Scientific and Statistical Computing 7.3 (1986), pp. 856-869. DOI: 10 . 1137/0907058. eprint: http://dx.doi.org/10.1137/0907058. URL: http: //dx.doi.org/10.1137/0907058.

[70] Kane S. Yee. "Numerical solution of initial boundary value problems involving Maxwell's equations in isotropic media". In: IEEE Trans. Antennas and Propagation (1966), p. 302307.

[71] Karl S. Kunz and Raymond J Luebbers. Finite Difference Time Domain Method for Electromagnetics. CRC Press, 2000. 
[72] R. Courant, K. Friedrichs, and H. Lewy. "On the Partial Difference Equations of Mathematical Physics". In: IBM J. Res. Dev. 11.2 (Mar. 1967), pp. 215-234. ISSN: 0018-8646. DOI: 10.1147/rd.112.0215. URL: http://dx.doi.org/10. $1147 /$ rd. 112.0215

[73] A. C. Cangellaris and D. B. Wright. "Analysis of the numerical error caused by the stair-stepped approximation of a conducting boundary in FDTD simulations of electromagnetic phenomena". In: IEEE Transactions on Antennas and Propagation 39.10 (Oct. 1991), pp. 1518-1525. ISSN: 0018-926X. DOI: $10.1109 / 8.97384$.

[74] S. Dey and R. Mittra. "A locally conformal finite-difference time-domain (FDTD) algorithm for modeling three-dimensional perfectly conducting objects". In: IEEE Microwave and Guided Wave Letters 7.9 (Sept. 1997), pp. 273-275. ISSN: 1051-8207. DOI: $10.1109 / 75.622536$.

[75] C. Nieter, John R. Cary, Gregory R. Werner, David N. Smithe, and Peter H. Stoltz. "Application of Dey-Mittra conformal boundary algorithm to 3D electromagnetic modeling". In: Journal of Computational Physics 228.21 (2009), pp. 7902-7916. ISSN: 0021-9991. DOI: http://dx.doi.org/10.1016/j.jcp. 2009.07 .025. URL: http://www . sciencedirect.com/science/article/ pii/S0021999109003829.

[76] J. Boris. "Relativistic Plasma Simulation-Optimization". In: Proc. of the 4th Conf. on Numerical Simulation of Plasmas. Nov. 1970, pp. 3-67. DOI: 10 . 1109/EUMA . 1973. 331733. 
[77] W. C. Brown. "Description and Operating Characteristics of the Platinotron-A New Microwave Tube Device". In: Proceedings of the IRE 45.9 (Sept. 1957), pp. 1209-1222. ISSN: 0096-8390. DOI: 10.1109/JRPROC.1957.278526.

[78] G. E. Pokorny, A. E. Kushnick, and J. F. Hull. "The dematron - A new crossedfield amplifier". In: 1961 International Electron Devices Meeting. Oct. 1961, pp. 72-72. DOI: 10.1109/IEDM.1961.187248.

[79] O. Doehler, A. Dubois, and D. Maillart. "An M-type pulsed amplifier". In: Proceedings of the IEE - Part B: Radio and Electronic Engineering 105.10 (May 1958), pp. 454-456. DOI: 10.1049/pi-b-1.1958.0076.

[80] H. McDowell and A. Wilczek. "An L-band crossed-field amplifier chain". In: IEEE Transactions on Electron Devices 10.2 (Mar. 1963), pp. 100-100. ISSN: 0018-9383. DOI: 10.1109/T-ED.1963.15105.

[81] J. McBride and R. Springberg. "A high-power UHF crossed-field amplifier". In: IEEE Transactions on Electron Devices 16.2 (Feb. 1969), pp. 236-236. ISSN: 0018-9383. DOI: 10.1109/T-ED.1969.16614.

[82] N. S. Nicholls, L. Damon, and J. Collomb. "Long-pulse 500-kW wide-band crossed-field amplifier". In: IEEE Transactions on Electron Devices 16.2 (Feb. 1969), pp. 237-237. ISSN: 0018-9383. DOI: 10.1109/T-ED.1969.16618.

[83] K. Eppley, J. Feinstein, K. Ko, N. Kroll, T. Lee, et al. "A high power crossfield amplifier at X-band". In: Conference Record of the 1991 IEEE Particle Accelerator Conference. May 1991, 719-721 vol.2. DOI: 10.1109/PAC. 1991. 164418. 
[84] G. Cox, A. Reddish, D. W. Ward, and L. Damon. "Stability, Noise, Efficiency and Gain of High Power Injected Beam Crossed Field Amplifiers". In: Microwave Conference, 1973. 3rd European. Vol. 2. Sept. 1973, pp. 1-4. DOI: 10.1109/EUMA. 1973.331733.

[85] G. H. MacMaster. "Current status of crossed-field devices". In: Technical Digest., International Electron Devices Meeting. Dec. 1988, pp. 358-361. DOI: 10.1109/IEDM. 1988.32830 .

[86] A. S. Gilmour, G. K. Farney, and B. R. Gray. "RF tubes for space-based accelerators". In: IEEE Transactions on Electron Devices 38.10 (Oct. 1991), pp. 2190-2204. ISSN: 0018-9383. DOI: 10.1109/16.88499.

[87] G. S. Kino. "A New Type of Crossed-Field Electron Gun". In: Crossed-Field Microwave Devices. Ed. by E. Okress, G. Mourier, J. Feinstein, and E. Kettlewell. Vol. 1. London: Academic Press Inc., 1961, pp. 164-177.

[88] H. G. Kosmahl. "Modern multistage depressed collectors - A review". In: Proceedings of the IEEE 70.11 (Nov. 1982), pp. 1325-1334. ISSN: 0018-9219. DOI: $10.1109 /$ PROC. 1982.12481.

[89] R. P. Wadhwa and T. Van Duzer. "A 3.5-dB noise-figure, S-band, mediumpower, forward-wave, injected-beam crossed-field amplifier". In: Proceedings of the IEEE 53.4 (Apr. 1965), pp. 425-426. ISSN: 0018-9219. DOI: 10.1109/PROC. 1965.3799.

[90] G. G. Groshart and R. R. Moats. "Multimode injected-beam crossed-field amplifier for coherent airborne radar". In: Electron Devices Meeting, 1980 International. Vol. 26. 1980, pp. 173-175. DOI: 10.1109/IEDM.1980.189785. 
[91] G. Döhler. "Beam injection control in broad band injected-beam crossed-field amplifiers". In: Electron Devices Meeting (IEDM), 1974 International. Dec. 1974, pp. 201-204. DOI: 10.1109/IEDM. 1974.6219647.

[92] Ronald M. Gilgenbach, Yue-Ying LAU, Hunter McDowell, Keith L. Cartwright, and Thomas A. Spencer. "Crossed-Field Devices". In: Modern Microwave and Millimeter-Wave Power Electronics. Wiley, 2005. Chap. 6.

[93] Sulmer Fernandez-Gutierrez, Jim Browning, Ming-Chieh Lin, David N Smithe, and Jack Watrous. "Simulation of a rising sun magnetron employing a faceted cathode with a continuous current source". In: Journal of Vacuum Science E Technology B 32.6 (2014), p. 061205.

[94] Sulmer Fernandez-Gutierrez, Jim Browning, Ming-Chieh Lin, David N Smithe, and Jack Watrous. "Phase-control of a rising sun magnetron using a modulated, addressable, current source". In: Journal of Vacuum Science 65 Technology B 33.3 (2015), p. 031203.

[95] Sulmer Fernandez-Gutierrez, Jim Browning, Ming-Chieh Lin, David N Smithe, and Jack Watrous. "Dynamic phase-control of a rising sun magnetron using modulated and continuous current". In: Journal of Applied Physics 119.4 (2016), p. 044501.

[96] A. G. Rozhnev, N. M. Ryskin, D. V. Sokolov, D. I. Trubetskov, S. T. Han, et al. "Novel concepts of vacuum microelectronic microwave devices with field emitter cathode arrays". In: Physics of Plasmas 9.9 (2002), pp. 4020-4027. DOI: http://dx.doi.org/10.1063/1.1497684. URL: http://scitation . aip.org/content/aip/journal/pop/9/9/10.1063/1.1497684. 
[97] J. P. Calame, H. F. Gray, and J. L. Shaw. "Analysis and design of microwave amplifiers employing field-emitter arrays". In: Journal of Applied Physics 73.3 (1993), pp. 1485-1504. DOI: http://dx.doi.org/10.1063/1.353223. URL: http://scitation.aip.org/content/aip/journal/jap/73/3/10.1063/1. 353223.

[98] Mikhail Fuks and Edl Schamiloglu. "Rapid Start of Oscillations in a Magnetron with a "Transparent" Cathode". In: Phys. Rev. Lett. 95 (20 Nov. 2005), p. 205101. DOI: 10.1103/PhysRevLett.95.205101. URL: http://link.aps . org/doi/10.1103/PhysRevLett.95.205101.

[99] H. Makishima, H. Imurat, M. Takahashi, H. Fukui, and A. Okamoto. "Remarkable improvements of microwave electron tubes through the development of the cathode materials". In: Vacuum Microelectronics Conference, 199\%. Technical Digest., 1997 10th International. Aug. 1997, pp. 194-199. DOI: 10.1109/IVMC . 1997.627473.

[100] P. M. Lally, Y. Goren, and E. A. Nettesheim. "An X-band tuned amplifier with a field-emission cathode". In: IEEE Transactions on Electron Devices 36.11 (Nov. 1989), pp. 2738-2741. ISSN: 0018-9383. DOI: 10.1109/16.43781.

[101] M. A. Kopot, V. D. Yeryomka, and V. P. Dzyuba. "3-B Simulation of Magnetrons Having a Secondary-Emission Cathode Stimulated by Electrons from a Field Emitter". In: 2006 IEEE International Vacuum Electronics Conference held Jointly with 2006 IEEE International Vacuum Electron Sources. 2006, pp. 351-353. DOI: 10.1109/IVELEC. 2006.1666328.

[102] Eusebio Garate, Roger D. McWilliams, Donald E. Voss, Alexander L. Lovesee, Kyle J. Hendricks, et al. "Novel cathode for field-emission applications". In: 
Review of Scientific Instruments 66.3 (1995), pp. 2528-2532. DOI: 10.1063/1. 1146504. eprint: http://aip.scitation.org/doi/pdf/10.1063/1.1146504. URL: http: //aip.scitation.org/doi/abs/10.1063/1.1146504.

[103] D. Shiffler, M. Ruebush, M. LaCour, K. Golby, R. Umstattd, et al. "Emission uniformity and emission area of explosive field emission cathodes". In: Applied Physics Letters 79.18 (2001), pp. 2871-2873. DOI: 10.1063/1.1415408. eprint: http : / / aip. scitation .org/doi/pdf/10.1063/1.1415408. URL: http: //aip.scitation.org/doi/abs/10.1063/1.1415408.

[104] R. B. Miller. "Mechanism of explosive electron emission for dielectric fiber (velvet) cathodes". In: Journal of Applied Physics 84.7 (1998), pp. 3880-3889. DOI: $10.1063 / 1$.368567. eprint: http://aip.scitation.org/doi/pdf/10. 1063/1 . 368567. URL: http://aip. scitation .org/doi/abs/10 . 1063/1. 368567.

[105] D. Shiffler, M. LaCour, K. Golby, M. Sena, M. Mithcell, et al. "Comparison of velvet- and cesium iodide-coated carbon fiber cathodes". In: IEEE Transactions on Plasma Science 29.3 (June 2001), pp. 445-451. ISSN: 0093-3813. DOI: 10. $1109 / 27.928942$.

[106] X. Yuan, X. Li, Ying Huang, Wenjie Fu, Y. Yan, et al. "Research on a magnetron injection electron gun based on carbon nanotube cold cathode". In: 2014 27th International Vacuum Nanoelectronics Conference (IVNC). July 2014, pp. 55-56. DOI: 10.1109/IVNC.2014.6894756.

[107] M. C. Jones, V. B. Neculaes, Y. Y. Lau, R. M. Gilgenbach, and W. M. White. "Cathode priming of a relativistic magnetron". In: Applied Physics Letters 85.26 (2004), pp. 6332-6334. DOI: 10.1063/1.1841454. eprint: http://dx . 
doi . org/10 .1063/1.1841454. URL: http://dx . doi .org/10.1063/1 . 1841454.

[108] Jim Browning. personal communication. Jan. 1, 2016.

[109] DuPont 951 Grean Tape Product Description. http: //www2 . dupont .com/ MCM/en_US/assets/downloads/prodinfo/951LTCCGreenTape.pdf. 2001.

[110] MathWorks. http://www.mathworks.com/index.html/.

[111] B. McCowan. "Calculating slow-wave circuit parameters with HFSS". In: $A b$ stracts. International Vacuum Electronics Conference 2000 (Cat. No.00EX392). May 2000, pages. DOI: 10.1109/OVE:EC . 2000.847482.

[112] Jim Browning and Jack Watrous. "Faceted magnetron concept using field emission cathodes". In: Journal of Vacuum Science 6 Technology B, Nanotechnology and Microelectronics: Materials, Processing, Measurement, and Phenomena 29.2 (2011), 02B109. DOI: 10.1116/1.3546035. eprint: http: //dx.doi.org/10.1116/1.3546035. URL: http://dx.doi.org/10.1116/1. 3546035. 